\title{
GENERATION-RECOMBINATION \\ NOISE IN EXTRINSIC \\ PHOTOCONDUCTIVE DETECTORS
}

by

Thomas John Brukilacchio

\author{
Submitted in Partial Fulfillment \\ of the \\ Requirements for the Degree \\ MASTER OF SCIENCE
}

Supervised by Professor Robert W. Boyd

The Institute of Optics University of Rochester

Rochester, New York

1983 


\section{CURRICULUM VITAE}

The author was born on December 11, 1959 in Newport, New York. He graduated in 1978 from West Canada Valley Central School in Newport, New York, and received a Bachelor of Science degree in Optics from the University of Rochester in May of 1982. The author began his graduate research at the Institute of Optics of the University of Rochester in September of 1982. His work was supported through a research grant from Jet Propulsion Laboratory. The author's research was supervised by Dr. Robert W. Boyd. 


\section{ACKNOWLEDGEMENTS}

I wish to express my gratitude to Professor Robert $W$. Boyd for his continued support and guidance throughout my undergraduate and graduate studies. I also wish to thank the other members of the Quantum Electronics Group for their support throughout this research. Mark Skeldon's insight in the theory section and Steven Wilk's assistance with the cryostat and vacuum system are also acknowledged. The advice on infrared detection and the loan of equipment from Professor Judith Pipher of the Department of Physics and Astronomy is also highly appreciated.

This work was funded by NASA through a research program of the Jet Propulsion Laboratory, California Institute of Technology. I thank Donald Langford of Jet Propulsion Laboratory for providing a design for the preamplifier, and also for the donation of the detectors and feedback resistors.

I am especially thankful for the warmth and comradery of my close friends in and out of the University of Rochester community. Finally, I wish to convey my love and respect toward my family for their returned love and moral support throughout my college experiences. 


\section{ABSTRACT}

A complete theory of generation-recombination noise is developed for extrinsic photoconductive detectors. A statistical approach uses Master equation techniques to derive closed form equations relating the noise and response characteristics of extrinsic detectors. Specifically, this treatment provides equations for the mean and mean-square fluctuation in the number of carriers, the response time, mean carrier lifetime, responsivity, and the noise equivalent power. The theory accounts for both the thermal and photo-induced generation of carriers. Impurity compensation of the majority carrier is addressed in this theory and is shown to have a significant effect on the noise and the response characteristics of this class of detectors.

A Ge:Ga far infrared detection system is designed, built, and tested. Responsivity measurements are made in the presence and absence of background photon flux. Dark current noise measurements are taken as a function of the detector temperature. The noise and response characteristics of the detector are compared to the theory developed in this thesis and are shown to be in good agreement with this theory. 
TABLE OF CONTENTS

PAGE

CURRICULUM VITAE

ACKNOWLEDGEMENTS

ABSTRACT iv

LIST OF FIGURES vij

CHAPTER

1 INTRODUCTION 1

2 THEORY OF GENERATION-RECOMBINATION NOISE 4

2.1 Introduction 4

2.2 A Statistical Treatment of
Generation-Recombination Noise

2.3 The Fractional Ionization of Available
Donor Impurities

2.4 Graphical Representation of
Detector Properties

2.5 Conclusion 48

3 EXPERIMENTAL SETUP OF A Ge:Ga DETECTION SYSTEM 51

3.1 Introduction 51

3.2 The Ge:Ga Detector 51

3.3 Helium Dewar 57

3.4 Detector Electronics $\quad 60$

3.5 Filter and Window Materials 68 
4 EXPERIMENTAL RESULTS

4.1 Introduction

4.2 Initial Testing and System Optimization 72

4.3 Experimental Results 74

5 SUMMARY AND CONCLUSIONS 93 
FIGURE

2.1 Photoconductor Impurity Levels. 6

2.2 Impurity Compensation of Photoconductive Materials. 6

2.3 The Ionization of $\mathrm{N}$ Free Electrons. 8

2.4 Fractional ionization as a function of the
generation rate.

2.5 Thermal generation rate as a function of the dimensionless temperature.

2.6 Fractional ionization versus dimensionless temperature at the limits of the compensation ratio.

2.7 Fractional ionization versus dimensionless temperature for factors of $\Gamma_{0}$ at $\mathrm{N}_{\mathrm{a}} \mathrm{N}_{\mathrm{d}}=0$. The parameter $\Gamma_{0}$ has the value $1.33 \times 10^{4}$.

2.8 Mean-square carrier fluctuation versus fractional ionization.

2.9 Mean-square carrier fluctuation versus generation rate.

2.10 Mean-square carrier fluctuation versus dimensionless temperature.

2.11 Modified mean-square carrier fluctuation versus fractional ionization. It represents the degree to which the statistics are Poissonian.

2.12 Modified mean-square carrier fluctuation versus generation rate.

2.13 Modified mean-square carrier fluctuation versus dimensionless temperature. 
2.14 Normalized response time versus fractional ionization.

2.15 Normalized response time versus generation rate.

2.16 Normalized response time versus dimensionless temperature.

2.17 Normalized mean carrier lifetime versus fractional ionization.

2.18 Normalized mean carrier lifetime versus generation rate.

2.19 Normalized mean carrier lifetime versus dimensionless temperature.

2.20 Normalized responsivity versus fractional ionization.

2.21 Normalized responsivity versus generation rate.

2.22 Normalized responsivity versus dimensionless temperature.

2.23 Dimensionless NEP versus fractional ionization. 45

2.24 Dimensionless NEP versus generation rate. 46

$\begin{array}{lll}\text { 2.25 Dimensionless NEP versus dimensionless temperature. } & 47\end{array}$

3.1 Experimental Setup. 52

3.2 Relative Wavelength Response of Ge:Ga Detector. 54

3.3 Impurity levels in Ge:Ga at 2.5 degrees Kelvin. 55

3.4 Detector mount and integrating cavity. 56

3.5 Helium Dewar. 58

3.6 Cryostat cold shield and work surface. 59

$\begin{array}{lll}3.7 & \text { Block diagram of electronics. } & 61\end{array}$

3.8 Preamplifier circuit. 63

3.9 True rms-to-DC noise circuit with a gain of 100 . 66 
$\begin{array}{lll}3.10 & \text { Filter band-pass. } & 69\end{array}$

$\begin{array}{lll}4.1 & \text { Fractional ionization versus temperature. } & 77\end{array}$

$\begin{array}{lll}4.2 & \text { Dark current versus temperature. } & 78\end{array}$

4.3 SBRC Data for dark current versus temperature. 81

4.4 Mean-square dark current noise versus temperature. 82

4.5 Photocurrent versus blackbody temperature. 86

4.6 Photocurrent versus total incident power. 87

4.7 Current responsivity versus blackbody temperature. 89

4.8 Current responsivity versus total incident power. 90 
CHAPTER 1

INTRODUCTION

Generation-recombination noise constitutes the dominant noise mechanism in semiconductors and in photoconductive materials. This noise arises from the statistical fluctuation in the number of conduction-band electrons and valence-band holes that are available to conduct a current at any given time. Recent interest in highly sensitive photoconductive detectors has motivated the investigation of generation-recombination $(g-r)$ noise.

Pioneering groundwork that led to a treatment of $g-r$ noise in semiconductors was carried out by K.M. van Vliet ${ }^{1}$ and by R.E. Burgess $^{2}$ in the mid 1950's. Burgess showed that equivalent results could be derived from thermodynamic and statistical approaches to the fluctuation in the number of charge carriers in a semiconductor.

This thesis presents a complete theory of the generationrecombination noise associated with extrinsic photoconductive detectors. To the knowledge of the author, this thesis represents the most complete treatment of $g-r$ noise available in literature to date. Master equation techniques are employed in deriving closed form equa- 
tions for the bulk response characteristics of extrinsic photoconductive detectors. This theory accounts for both the thermal and photo-induced generation of charge carriers. Additionally, we address the effects on the response characteristics of a detector due to the impurity compensation of the majority carrier. The detector response functions plotted in chapter 2 indicate that impurity compensation can lead to dramatic differences in the response characteristics of extrinsic photoconductive detectors.

Within the scientific community, astronomers may benefit the most from a more complete understanding of $g-r$ noise theory, as they often observe under conditions of very low backgrounds. Recent astronomical interest in the space-based applications of 100 micron detection led to the availability of Ge:Ga detectors to the author. Chapters 3 and 4 of this thesis describe a 100 micron Ge:Ga detection system constructed by the author. Detector response measurements were found to be in good qualitative agreement with the theory of generation-recombination noise as presented in chapter 2. 


\section{REFERENCES}

1. K.M. van Vliet, "Noise in Semiconductors and Photoconductors," (IRE, March 3, 1958).

2. R.E. Burgess, "Fluctuations of the Numbers of Electrons and Holes in a Semiconductor," (Proceedings of the Physical Society of London, B 68, 1955). 
CHAPTER 2

THEORY OF GENERATION-RECOMBINATION NOISE

\subsection{Introduction}

This chapter presents a theory that is capable of producing explicit expressions for the statistical fluctuations in the number of charge carriers in a given volume of a photoconductive material. This theory takes proper account of the presence of both donor and acceptor impurities. A method of detailed balancing of the transition rates of electrons is employed in steady state to determine specific characteristics of photoconductive materials.

Both Burgess ${ }^{1}$ and van Vliet ${ }^{2}$ have presented fairly comprehensive treatments of fluctuations in impurity doped semiconductors, but neither takes proper account of the effect of compensating impurities on the response characteristics of the detector. As will be shown below, the effect of impurity compensation is often far from negligible.

Two alternate formulations of generation-recombination theory have been treated by the aforementioned authors: a statistical 
approach and a thermodynamic approach. The present analys is will follow the statistical approach, because this approach allows us to treat a system that is in the steady state, but not necessarily in thermodynamic equilibrium. Thermodynamic arguments will be introduced only to account for the rate at which carriers are generated by thermal processes. The present model should thus be well suited to the treatment of radiation detectors.

Before developing the theory, it is instructive to illustrate the processes that take place in an impurity-compensated photoconductive detector by means of energy band diagrams. We must point out that gallium is an acceptor impurity in Ge: Ga, the detector material used in the experimental work also reported in this thesis. The following analysis, however, will refer to an n-type photoconductor because it is easier to conceptualize the motion of electrons than that of holes. This convention is commonly employed in the literature on this subject. In the ensuing discussion we will assume that the detector temperature is low enough that band to band transitions can be ignored.

Figure (2.1) depicts an impurity photoconductor containing a total of $N_{d}$ donor impurity sites lying close to the bottom of the conduction band and also containing a total of $\mathrm{N}_{\mathrm{a}}$ acceptor impurity sites lying within the bandgap, close to the top of the valance band. Since we are considering an n-type photoconductor, the total number of donor impurities is greater than the total number of acceptor impurities. 
CB

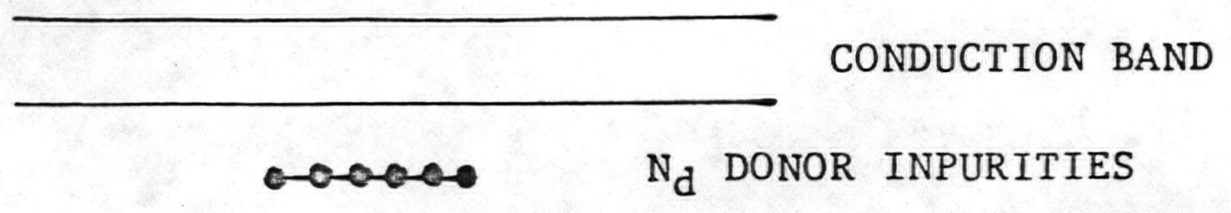

$\theta 0 \theta \quad \mathrm{N}_{\mathrm{a}}$ ACCEPTOR INPURITIES

VB

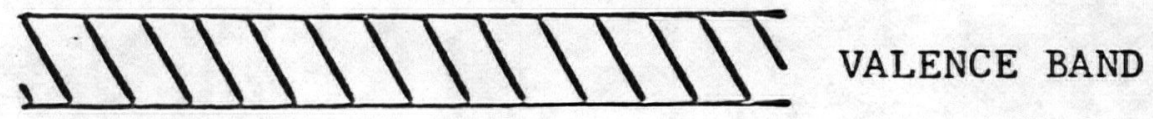

Fig(2.1) - Photoconductor Inpurity Levels

CB

CONDUCTION BAND

N $\mathrm{N}_{a}$ OF THE $\mathrm{N}_{d}$ DONOR INPURITIES IONIZED

$\checkmark \mathrm{Na}_{\mathrm{a}}$ ACCEPTOR IMPURITIES COMPLETELY IONIZED

VB

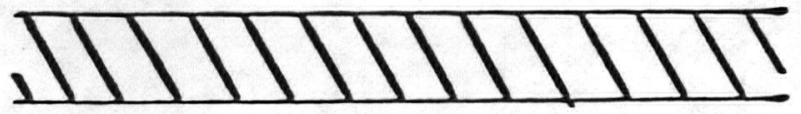

VALENCE BAND

Fig(2.2) - Impurity Compensation of Photoconductive Materials 
Figure (2.2) illustrates that $\mathrm{N}_{\mathrm{a}}$ of the $\mathrm{N}_{\mathrm{d}}$ donor impurities are ionized even at the temperature of absolute zero. This phenomenon is known as compensation since only $\mathrm{N}_{d}-\mathrm{N}_{a}$ of the donor impurities are available for optical excitation to the conduction band.

As illustrated by Figure (2.3), we let $\mathrm{N}$ denote the total number of free electrons within the conduction band. Hence $\mathrm{N}_{a}+\mathrm{N}$ of the $\mathrm{N}_{d}$ donor impurities are ionized and therefore available as recombination centers. Likewise, $\mathrm{N}_{d}-\mathrm{N}_{\mathrm{a}}-\mathrm{N}$ of the $\mathrm{N}_{\mathrm{d}}$ donor impurities are un-ionized and available for excitation to the conduction band. Electrons can be excited from the donor levels to the conduction band through both thermal-excitation and photo-excitation.

\subsection{A Statistical Treatment of Generation-Recombination Noise}

Generation-recombination noise results from the time varying statistical fluctuation in the number of free (i.e., conduction band) charge carriers. ${ }^{3}$ These statistical fluctuations can be calculated from the transition rates of electrons between the conduction band and the donor impurity level. The present analysis considers only one independently fluctuating variable, in this case the number of conduction band electrons $\mathrm{N}$. The validity of this assumption has been illustrated in figure (2.2). Thus, we are considering a two level system in that only two of the levels of figure (2.2) interact dynamically with the optical radiation.

The method of detailed balancing of the generation and recombination rates is applied to determine the statistical fluctuation in 


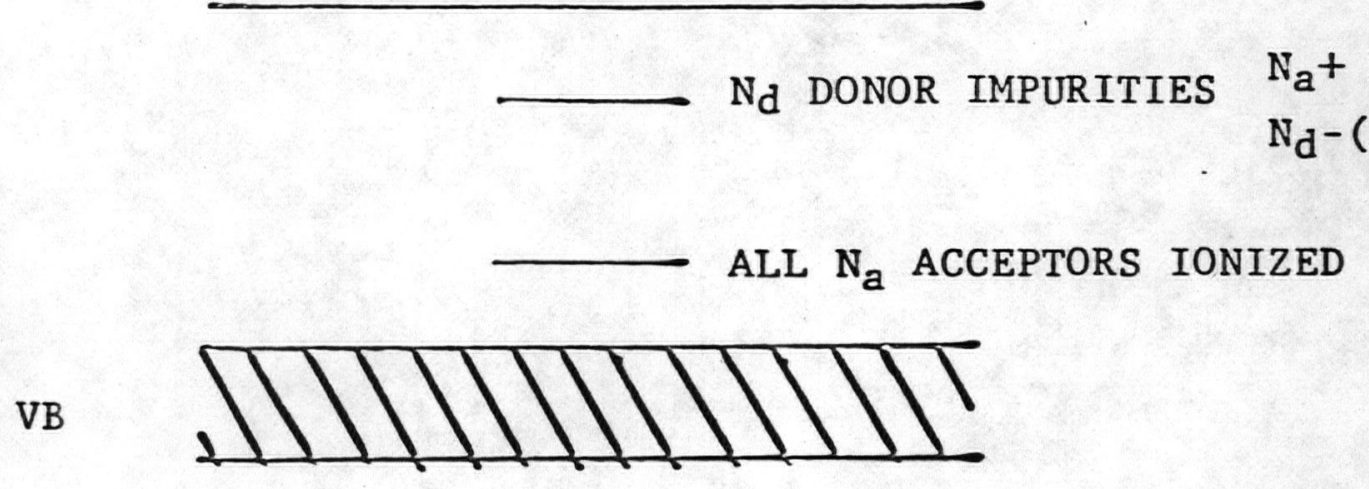

Fig(2.3) - The Ionization of N Free Electrons 
the number of conduction band electrons, as well as the mean-square carrier fluctuation, response time, mean carrier lifetime, and the mean-square $g-r$ current noise (henceforth $g-r$ will denote generationrecombination).

The transition probabilities per unit time of electrons between the donor level and the conduction band are defined as follows: $\mathrm{g}(\mathrm{N}) \mathrm{dt}$ is the probability that one additional electron is excited to the conduction band in a time $\mathrm{dt}$ if $\mathrm{N}$ electrons are already there. The generation rate $g(N)$ is assumed to be proportional to the product of the number of un-ionized donors $\mathrm{N}_{d}-\mathrm{N}_{a}-\mathrm{N}$ with the generation coefficient $\gamma$ as follows:

$$
g(N)=\gamma\left(N_{d}-N_{a}-N\right) .
$$

The generation coefficient $\gamma$ is assumed to be the sum of a thermal contribution that increases rapidly with temperature and a radiative contribution that increases linearly with photon flux. Similarly, a recombination rate $r(N)$ is defined such that $r(N) d t$ is the probability that within a time interval $\mathrm{dt}$ one of the $\mathrm{N}$ electrons initially in the conduction band returns to the donor level. The recombination rate $r(N)$ is assumed to be of the form

$$
r(N)=\rho N\left(N_{a}+N\right),
$$

where $\mathrm{N}_{\mathrm{a}}+\mathrm{N}$ is the number of recombination sites (i.e., the number of ionized donors), and $\rho$ is the recombination coefficient which may be weakly temperature dependent. In his thermodynamic treatment of $g-r$ noise, Burgess ${ }^{4}$ shows that $\rho$ is of the form $\overline{v S} / V$ where $V$ is the 
volume of the sample, $\bar{v}$ is the mean thermal velocity of a conduction band electron, and $\bar{S}$ is the mean cross section for capture by a positive ion.

It was first shown by Burgess ${ }^{5}$ that master equation techniques can be used to determine the mean number and the mean-square fluctuation in the number of carriers present in a doped semiconductor. His analysis assumes that the functional forms of the generation and recombination rates are known and that they depend on only one fluctuating variable which, as discussed above, we take to be $N$. The master equation describes the time evolution of the probability distribution function $p(N)$, which denotes the probability that the conduction band contains $\mathrm{N}$ electrons. The time rate of change of $p(N)$ is given by [see for instance ref. 6]

$\frac{d}{d t} p(N)=r(N+1) p(N+1)+g(N-1) p(N-1)-p(N)[g(N)+r(N)]$.

Under steady state conditions $d p(N) / d t$ is equal to zero and the equation (2.3) can be solved iteratively to obtain

$$
\frac{p(N)}{p(0)}=\prod_{v=0}^{N-1} g(v) / \pi_{v=1}^{N} r(v) .
$$

The most probable value $\bar{N}$ of $N$ can be found by logarithmic differentiation of $p(N)$ with respect to $N$ and then equating the derivative to zero: 


$$
\frac{d}{d N} \ln p(N)=\frac{\ln g(\bar{N})}{\ln r(\bar{N}+1)}=0
$$

It is assumed that $\bar{N}$ is large compared to unity. As expected, the equilibrium condition of equation (2.5) leads to equal generation and recombination rates:

$$
g(\bar{N})=r(\bar{N})
$$

The variance in $\mathrm{N}$ can be found by expanding $\ln \mathrm{p}(\mathrm{N})$ in a power series centered on $N=\bar{N}$ :

$$
\begin{aligned}
\ln p(N)= & \ln p(\bar{N})-\left.(N-\bar{N}) \frac{d}{d N} \ln p(N)\right|_{N=\bar{N}}+\ldots . \\
& +\left.\frac{1}{2}(N-\bar{N})^{2} \frac{d^{2}}{d N^{2}} \ln p(N)\right|_{N=\bar{N}}+\ldots .
\end{aligned}
$$

where the second term will vanish and terms beyond the third can be neglected. Calculation of this last derivative leads to the following gaussian distribution of $N$ about $\bar{N}$ :

$$
\begin{aligned}
\left.\frac{d^{2}}{d N^{2}} \ln p(N)\right|_{N=\bar{N}} & =\left.\frac{d}{d N}\left(\frac{d}{d N} \ln p(N)\right)\right|_{N=\bar{N}} \\
& =\left.\frac{d}{d N}(\ln g(N)-\ln r(N))\right|_{N=\bar{N}} \\
& =\frac{g^{\prime}(\bar{N})}{g(\bar{N})}-\frac{r^{\prime}(\bar{N})}{r(\bar{N})},
\end{aligned}
$$


where the prime denotes differentiation with respect to $N$. Substitution of the gaussian distribution of equation (2.10) into the power series expansion of $\ln p(N)$ of equation (2.7) results in:

$$
\ln p(N)=\ln p(\bar{N})-\frac{1}{2}(N-\bar{N})^{2}\left[\frac{r^{\prime}(\bar{N})}{r(\bar{N})}-\frac{g^{\prime}(\bar{N})}{g(\bar{N})}\right]
$$

or equivalently

$$
p(N)=p(\bar{N}) \exp \left[\frac{-(N-\bar{N})^{2}}{2(\Delta N)^{2}}\right]
$$

where the variance of $\mathrm{N}$ is given by

$$
\overline{(\Delta N)^{2}}=\frac{g(\bar{N})}{r^{\prime}(\bar{N})-g^{\prime}(\bar{N})} .
$$

The relevance of the denominator of equation (2.13) is understood by considering the time evolution of the system returning to equilibrium after it has been perturbed by an amount $N-\bar{N}$. The expectation value of the rate of change of $N$ towards $\bar{N}$ is

$$
\overline{\frac{d}{d t}(N-\bar{N})}=\frac{\overline{d N}}{d t}=g(N)-r(N),
$$

where for small deviations

$$
\overline{d N} \cong\left[r^{\prime}(\bar{N})-g^{\prime}(\bar{N})\right](N-\bar{N}) \text {. }
$$


Thus, the system relaxes to equilibrium according to

$$
\Delta N(t)=\Delta N(0) \exp \left(-t / \tau^{\prime}\right),
$$

where $\tau^{\prime}$ is the relaxation time given by

$$
\tau^{\prime}=\frac{1}{r^{\prime}(\bar{N})-g^{\prime}(\bar{N})} \text {. }
$$

$\tau^{\prime}$ is interpreted as the response time of the photoconductor or as the incremental carrier lifetime, that is, the lifetime of an additional carrier when $\mathrm{N}$ are known to be already present. ${ }^{7}$

The response time is not to be confused with the mean carrier lifetime $\bar{\tau}$ which is defined as the difference between the mean number of conduction band electrons and the mean number that would exist if the generation rate were zero. Thus, the mean carrier lifetime is given by

$$
\bar{\tau}=\frac{\bar{N}}{g(\bar{N})} .
$$

With the use of equation (2.17), the variance in $\mathrm{N}$ can be expressed as

$$
\overline{(\Delta N)^{2}} \quad=g(\bar{N}) \tau^{\prime} .
$$

This result was first obtained by Burgess ${ }^{8}$ and is known as the $\mathrm{g}-\mathrm{r}$ theorem.

To arrive at an expression for the mean-square $g-r$ current noise, consider a slab of photoconductive material of cross sectional area $A$ and length $L$ to which a potential of magnitude $V$ has been 
applied. Most generally, the instantaneous value of the current passing through the photoconductive material is related to the total number of free electrons $N(t)=A L n(t)$ and to the total number of free holes $P(t)=A L p(t)$, where $n(t)$ and $p(t)$ are the electron and hole densities respectively, by

$$
i(t)=\frac{e V}{L^{2}}\left[\mu_{n} N(t)+\mu_{p} P(t)\right]
$$

where $\mu_{n}$ and $\mu_{p}$ are the electron and hole carrier mobilities respectively, and $e$ is the fundamental electric charge. The mean value of this current is given by

$$
\bar{i}=\frac{e V}{L^{2}}\left[\mu_{n} \bar{N}+\mu_{p} \bar{P}\right]
$$

The fluctuation in the current $\Delta i=i(t)-\bar{i}$ is thus directly related to the fluctuations in the carrier concentrations $\Delta N=N(t)-\bar{N}$ and $\Delta P=P(t)-\bar{P}$ by

$$
\overline{(\Delta i)^{2}}=\frac{e^{2} v^{2}}{L^{2}}\left[\mu_{n}^{2} \overline{(\Delta N)^{2}}+2 \mu_{n} \mu_{p} \overline{(\Delta N)(\Delta P)}+\mu_{p}^{2} \overline{(\Delta P)^{2}}\right] .
$$

This relation is expressed in terms of $\bar{i}$ and the mobility ratio $b=\mu_{n} / \mu_{p}$ by

$$
\overline{(\Delta i)^{2}}=\overline{i_{g r}^{2}}=\left(\frac{\bar{i}}{b \bar{N}+\bar{P}}\right)^{2}\left[b^{2} \overline{(\Delta N)^{2}}+2 b \overline{(\Delta N)(\Delta P)}+\overline{(\Delta P)^{2}}\right],
$$

where $i_{g r}$ is used to designate the generation-recombination current noise. ${ }^{9}$ 
The spectral density of the current fluctuations will be given by

$\overline{i_{g r}^{2}}(f)=\left(\frac{\bar{i}}{b \bar{N}+\bar{P}}\right)^{2}\left[b^{2} \overline{(\Delta N)^{2}}(f)+2 \overline{(\Delta N)(\Delta P)}(f)+\overline{(\Delta P)^{2}}(f)\right]$.

It can be concluded from equations (2.17) and (2.19) that the correlation function of $\mathrm{N}$ is

$$
C_{N}(t)=g(\bar{N}) \tau^{\prime} \exp \left(-t / \tau^{\prime}\right)
$$

The spectral density of the fluctuation in $\mathrm{N}$ is obtained from the Wiener-Khintchine theorem as

$$
\overline{(\Delta N)^{2}}(f)=\frac{4 g(\bar{N}) \tau^{\prime 2}}{1+\left(2 \pi f \tau^{\prime}\right)^{2}} .
$$

Under the condition that $f \ll 1 / 2 \pi \tau^{\prime}$, the spectral density of $N$ reduces to

$$
\overline{(\Delta N)^{2}}(f)=4 g(\bar{N}) \tau^{\prime 2}
$$

When an extrinsic photoconductor with a majority of donor impurities is considered, as in the present analysis, $\Delta N \gg \Delta P$ and $\bar{N} \gg \bar{P}$, reducing equation (2.24) to

$$
\overline{i_{g r}^{2}}(f)=\frac{\bar{i}^{2}}{\bar{N}^{2}} \overline{(\Delta N)^{2}}(f) .
$$

It follows from equation (2.27) that

$$
\overline{i_{g r}^{2}}(f)=\frac{4 \bar{i}^{2}}{\bar{N}^{2}} g(\bar{N}) \tau^{\prime 2},
$$


and from equation (2.19) that

$$
\overline{i_{g r}^{2}}(f)=\frac{4 \bar{i}^{2}}{\bar{N}^{2}} \overline{(\Delta N)^{2}} \tau^{\prime}
$$

Thus, the mean-square $g-r$ current noise is given by the product of the spectral density of the current fluctuations and the electrical bandwidth $\Delta f$ by ${ }^{10}$

$$
\overline{i_{g r}^{2}}=4 \frac{\bar{i}^{2}}{\bar{N}^{2}} \overline{(\Delta N)^{2}} \tau^{\prime} \Delta f .
$$

2.3 The Fractional Ionization of Available Donor Impurities

An explicit expression for the mean number $\overline{\mathrm{N}}$ of conduction band electrons will now be derived. Equating equations (2.1) and (2.2) under conditions of equilibrium and solving fo $\overline{\mathrm{N}}$ results in:

$$
\left.\bar{N}=\frac{-1}{2}\left(\frac{\gamma}{\rho}+N_{a}\right)+\left[\frac{1}{4}\left(\frac{\gamma}{\rho}+N_{a}\right)^{2}+\frac{\gamma}{\rho}\left(N_{d}-N_{a}\right)\right)\right]^{\frac{1}{2}} .
$$

It is convenient to describe the properties of the detector in terms of the fractional ionization of available donor impurities. This quantity is given by the ratio of the mean total number of carriers to the total number of available donor impurities as

$$
f=\frac{\bar{N}}{N_{d}-N_{a}}=\frac{\bar{N}}{N_{d}}\left(\frac{1}{1-\lambda}\right),
$$


where

$$
\lambda=\frac{\mathrm{N}_{\mathrm{a}}}{\mathrm{N}_{\mathrm{d}}} .
$$

$\lambda$ is referred to as the impurity compensation ratio. Thus, the fractional ionization is given by

$$
f=\left(\frac{1}{1-\lambda}\right)\left\{\frac{-1}{2}\left(\frac{\gamma}{\rho N_{d}}+\lambda\right)+\left[\frac{1}{4}\left(\frac{\gamma}{\rho N_{d}}+\lambda\right)^{2}+\frac{\gamma}{\rho N_{d}}(1-\lambda)\right]^{\frac{1}{2}}\right\} \text {. }
$$

We define $\gamma / \rho N_{d}$ in equation (2.34) to be the dimensionless generation rate $\Gamma$ :

$$
\Gamma=\frac{\gamma}{\rho N_{d}} .
$$

This dimensionless generation rate has both radiative and thermal contributions:

$$
\Gamma=\Gamma_{\text {rad }}+\Gamma_{\text {th }} .
$$

Because the present analysis is a statistical approach, there has been no provision made for how $\Gamma$ scales with detector temperature. R.A. Smith, however, has derived an expression for the thermal generation of carriers through thermodynamic considerations. ${ }^{11}$ His treatment agrees with equation (2.32) when a dimensionless thermal generation rate $\Gamma_{\text {th }}$ is defined as

$$
\Gamma_{\text {th }}=\frac{V}{N_{d}} \frac{1}{2} N_{c} \exp \left(-\varepsilon_{d} / k T\right) \text {, }
$$


where $V$ is the detector volume, $\varepsilon_{d}$ is the donor energy with respect to the conduction band, $k$ is Boltzmann's constant, and $T$ is the temperature in degrees Kelvin. $N_{C}$ may be interpreted as the effective number of levels per unit volume in the conduction band. If it is assumed that the conduction band is parabolic, $N_{c}$ is given by

$$
N_{c}=2\left(2 \pi m k T / h^{2}\right)^{3 / 2} \text {, }
$$

where $m$ is the effective mass of the electron and $h$ is Planck's constant.

To assure that the present theory is applicable to any system, a dimensionless temperature is defined as

$$
\theta=k T / \varepsilon_{d}
$$

We also define a material-dependent proportionality constant $\Gamma_{0}$ as

$$
\Gamma_{0}=\frac{V}{N_{d}}\left(\frac{2 \pi m \varepsilon}{h^{2}}\right)^{3 / 2} .
$$

Thus, the dimensionless thermal generation rate can be expressed as

$$
\Gamma_{\text {th }}=\Gamma_{0} \theta^{3 / 2} \exp (-1 / \theta)
$$

\subsection{Graphical Representation of Detector Properties}

Much intuition into detector dynamics is gained by representing graphically the quantities derived above as functions of $f, \Gamma$, and $\mathrm{kT} / \varepsilon_{\mathrm{d}}$. In addition, two figures of merit that are used to evaluate 
the quality of a detector are derived and plotted; the responsivity $R$ and the noise equivalent power (NEP). Those plots that are functions of temperature, with the exception of figure (2.5), are unique to the Ge: Ga detectors described in Chapter 3 of this thesis, taking into account the detectors volume, doping level, and bandgap. All other plots are general to any extrinsic photoconductive detection system.

First, it is important to understand how the bulk detector properties depend on the operating conditions. Figure (2.4) depicts how the fractional ionization scales with the generation rate as shown by equation (2.34). Note that the fractional ionization saturates toward the value unity. This is in contrast with the case of a two-level atomic system, for which the maximum ionization is only one half.

Figure (2.5) illustrates how the thermal generation rate scales with temperature as given by equation (2.41). Notice that the material dependent term $\Gamma_{0}$ has been factored out for generality. Substitution of equation (2.41) into equation (2.34) yields an expression for the fractional ionization as a function of the dimensionless temperature. Figures (2.6) and (2.7) illustrate this functional form. The value $\Gamma_{0}=1.33 \times 10^{4}$, which is typical of Ge:Ga, is used here as well as in the remaining plots in this chapter which are functions of temperature. The temperature for which $\mathrm{kT} / \varepsilon_{\mathrm{d}}=0.4$ corresponds to approximately 50 degrees Kelvin for the Ge:Ga detector described in Chapter 3. Figure (2.6) illustrates that higher compensation ratios allow for higher operating temperatures. 


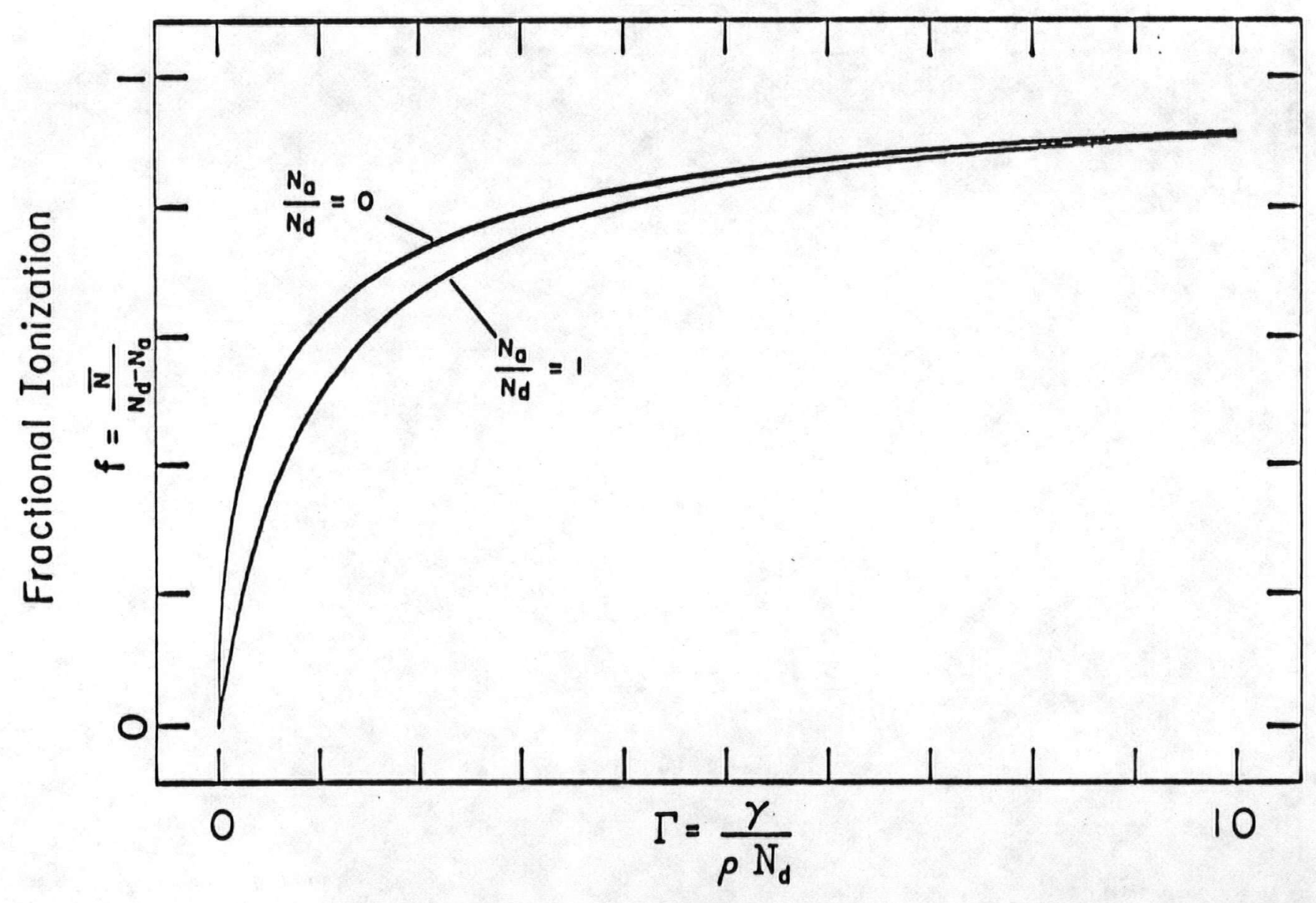

Generation Rate

Fig.(2.4) - Fractional ionization as a function of the generation rate. 


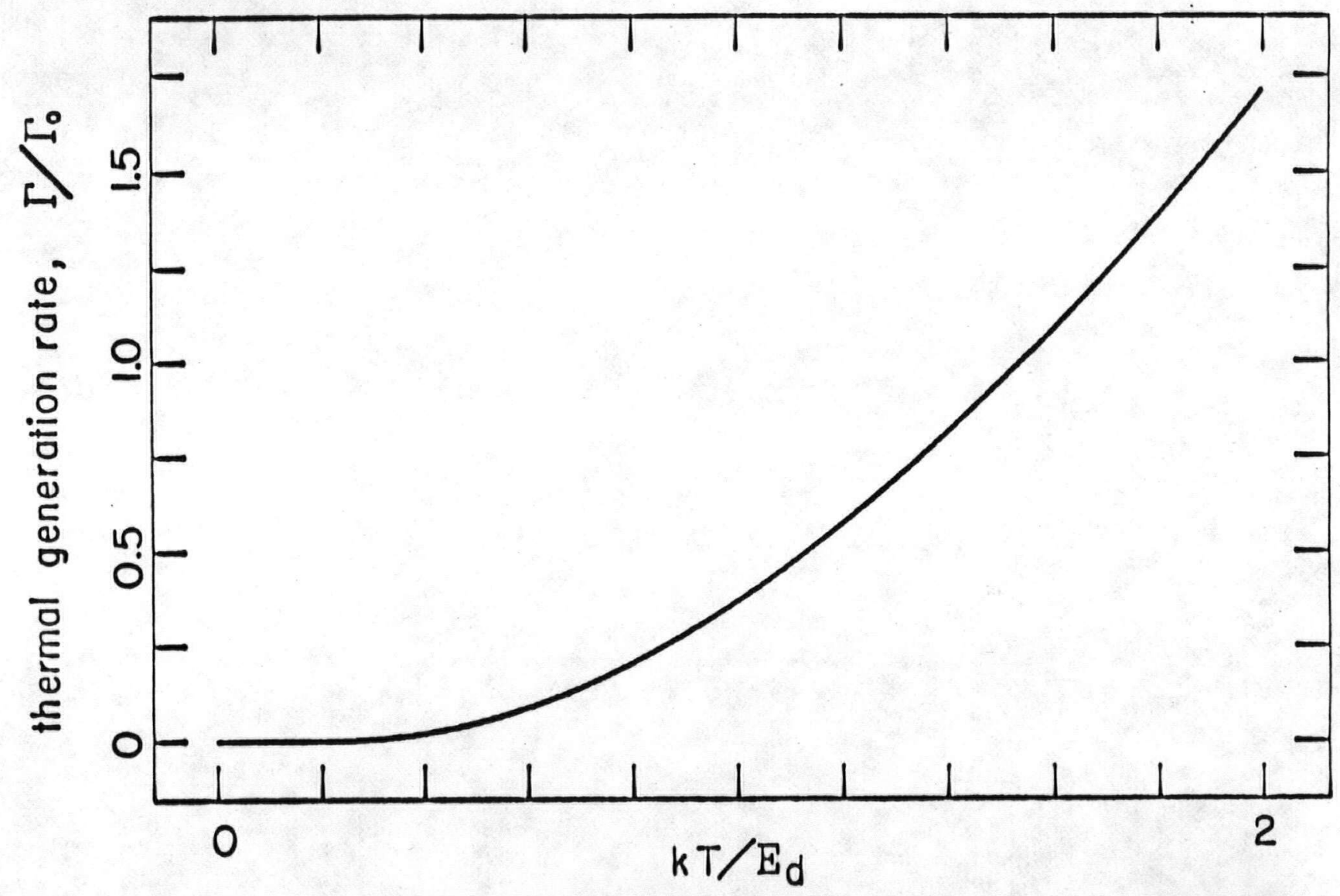

dimensionless temperature

Fig.(2.5) - Thermal generation rate as a function of the dimensionless temperature. 


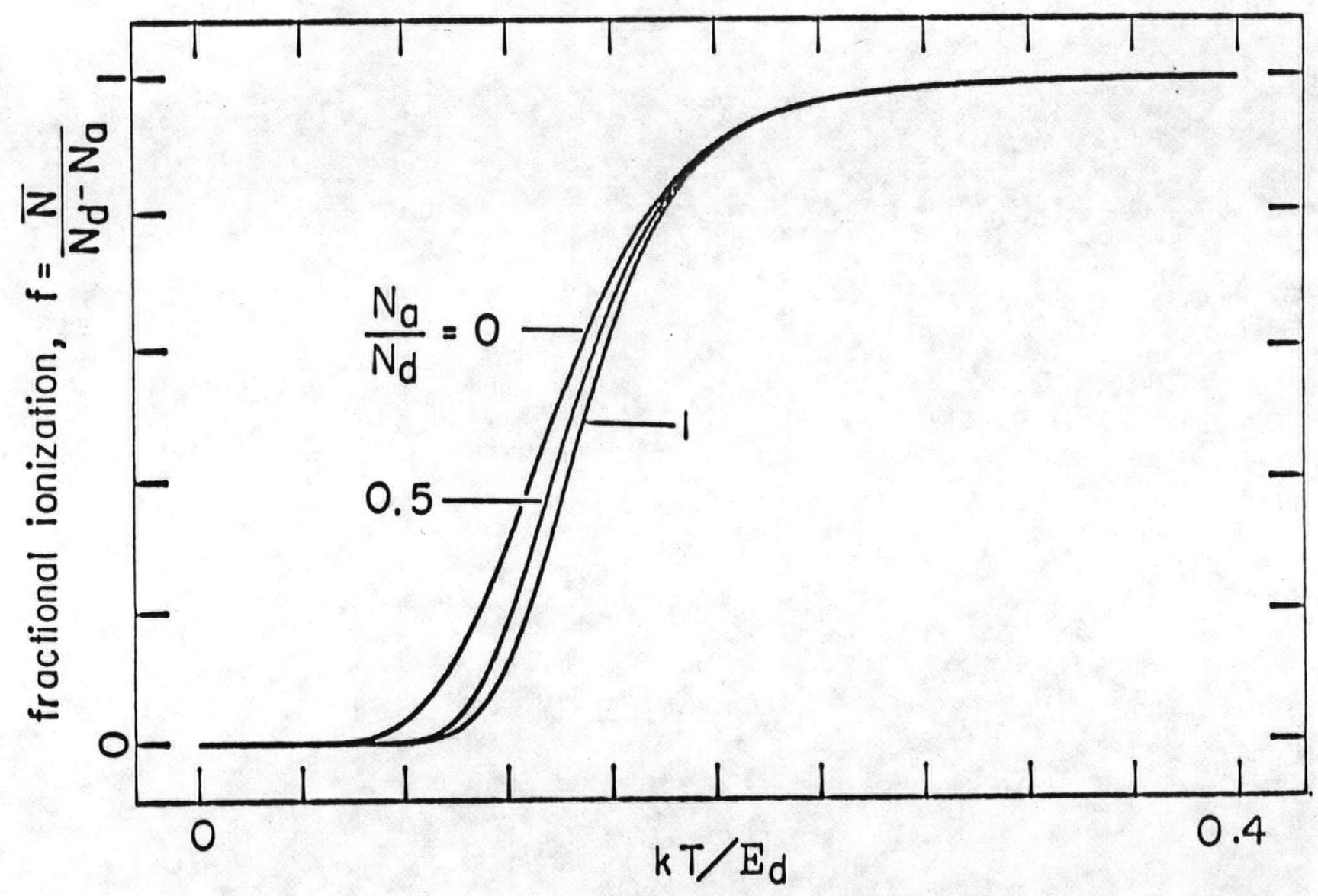

dimensionless temperature

Fig.(2.6) - Fractional ionization versus dimensionless temperature at the limits of the compensation ratio 


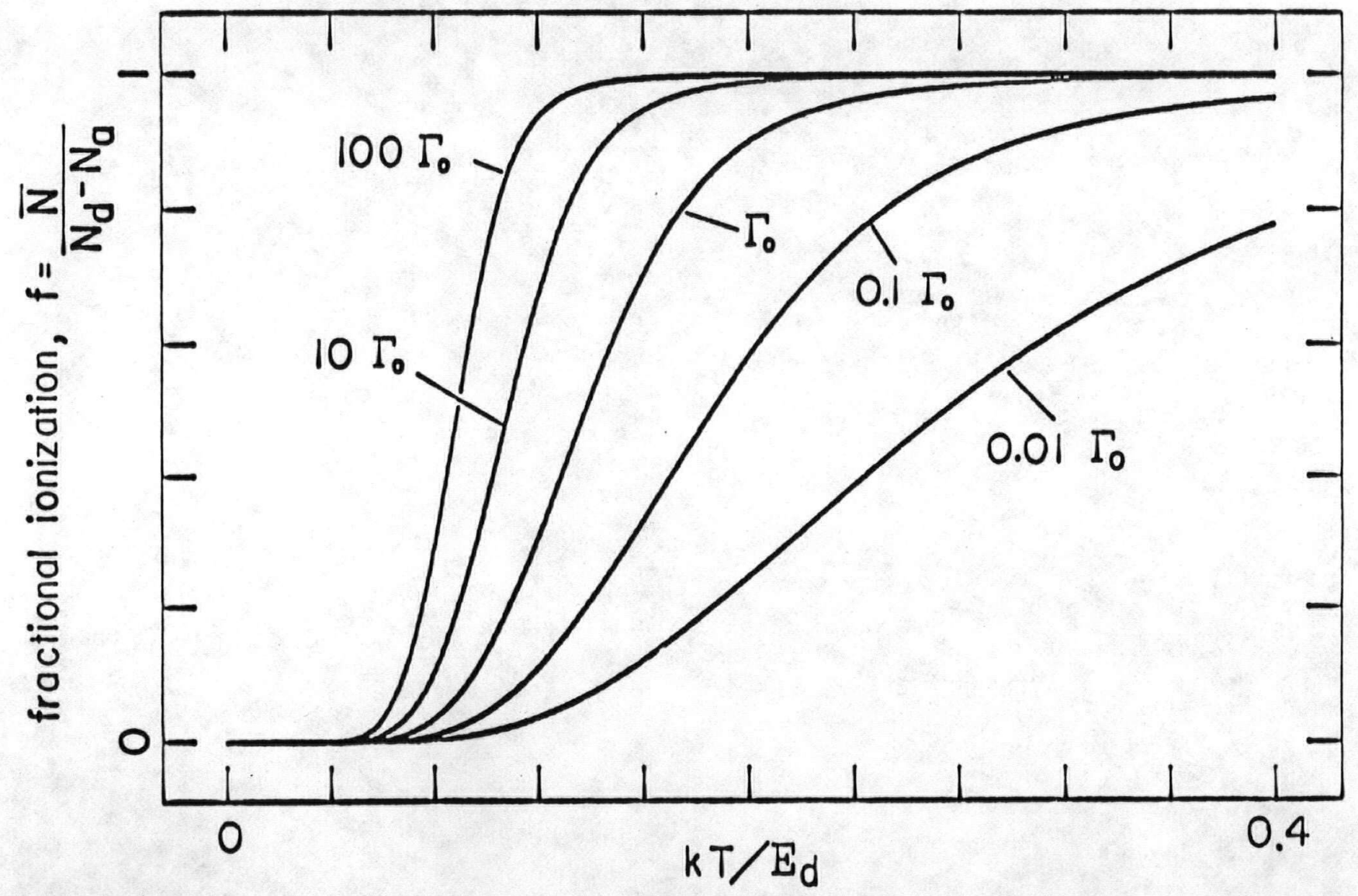

dimensionless temperature

Fig.(2.7) - Fractional ionization versus dimensionless temperature for factors of $\Gamma_{0}$ at $N_{a} / N_{d}=0$. The parameter $\Gamma_{0}$ has the value $1.33 \times 10^{4}$. 
The mean-square carrier fluctuation of equation (2.13) can be expressed in terms of the fractional ionization by substituting the differentiated values of equations (2.1) and (2.2) into equation (2.13) and by expressing it in terms of $f$ by using equation (2.33). This functional form,

$$
\overline{(\Delta N)^{2}}=N_{d} \frac{f(1-\lambda)(1-f)[\lambda+f(1-\lambda)]}{f(2-f)(1-\lambda)+\lambda},
$$

is illustrated in figure (2.8) for various compensation ratios. Figure (2.9) using equation (2.34), and figure (2.10) using equation (2.41), represent the mean-square current noise as functions of the generation rate and dimensionless temperature respectively. As witnessed by figure (2.10), the noise in the Ge:Ga system is appreciable between the temperatures of 4 and 5 degrees Kelvin.

Equation (2.42a) can be modified by dividing by the mean number of carriers $\bar{N}$ and then by expressing $\bar{N}$ in terms of $f$ by using equation (2.33), as

$$
\frac{\overline{(\Delta N)^{2}}}{\bar{N}}=\frac{(1-f)[\lambda+f(1-\lambda)]}{f(2-f)(1-\lambda)+\lambda} .
$$

This function can be interpreted as the degree to which the fluctuations are Poissonian in nature. If the fluctuations obeyed Poisson statistics, the quantity $\overline{(\Delta N)^{2}} / \bar{N}$ would have the value unity. These functions are illustrated in figures (2.11), (2.12), and (2.13).

By differentiating equations (2.1) and (2.2) for the generation and recombination rates at equilibrium and substituting into equation 


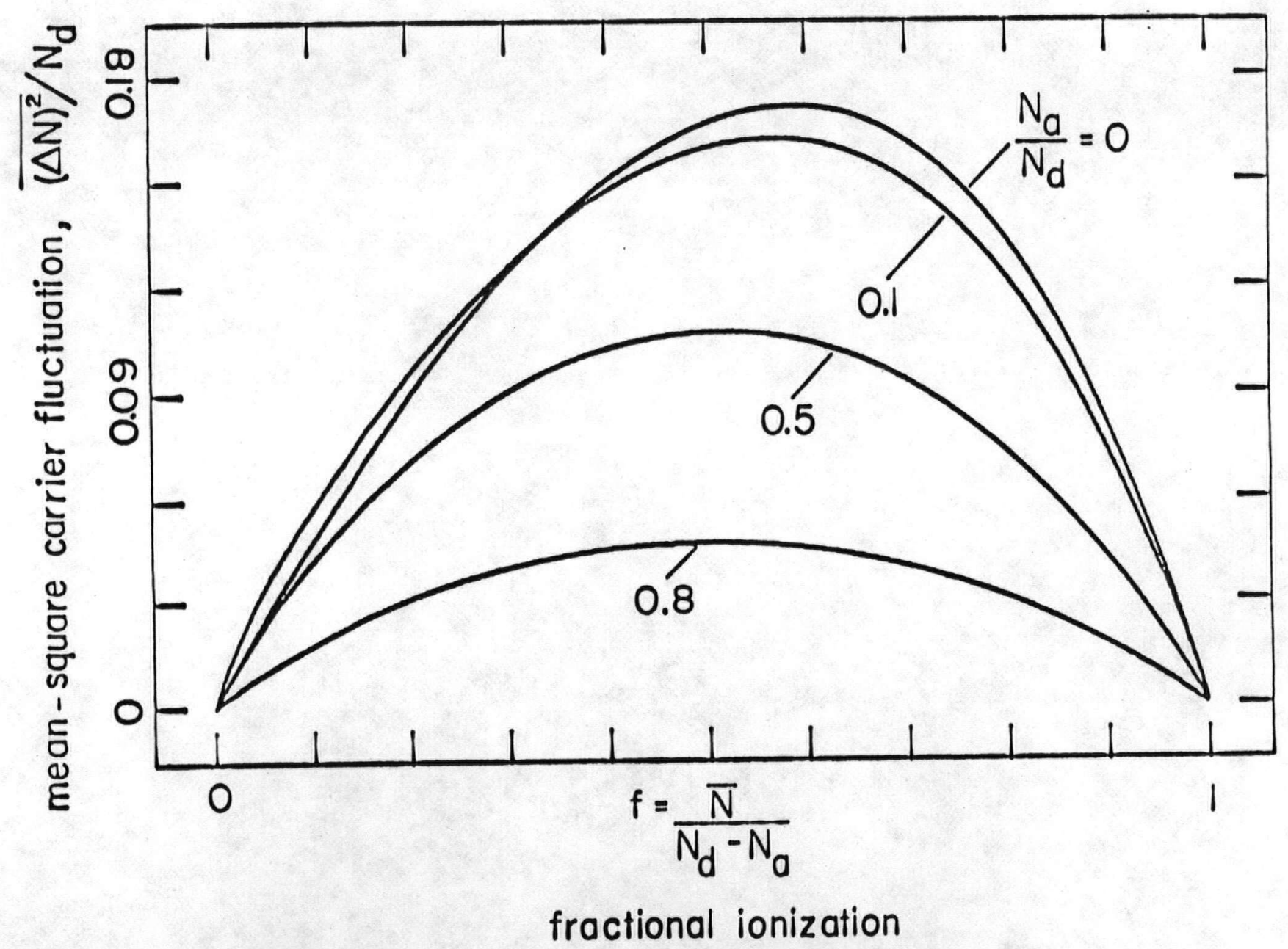

Fig.(2.8) - Mean-square carrier fluctuation versus fractional ionization. 


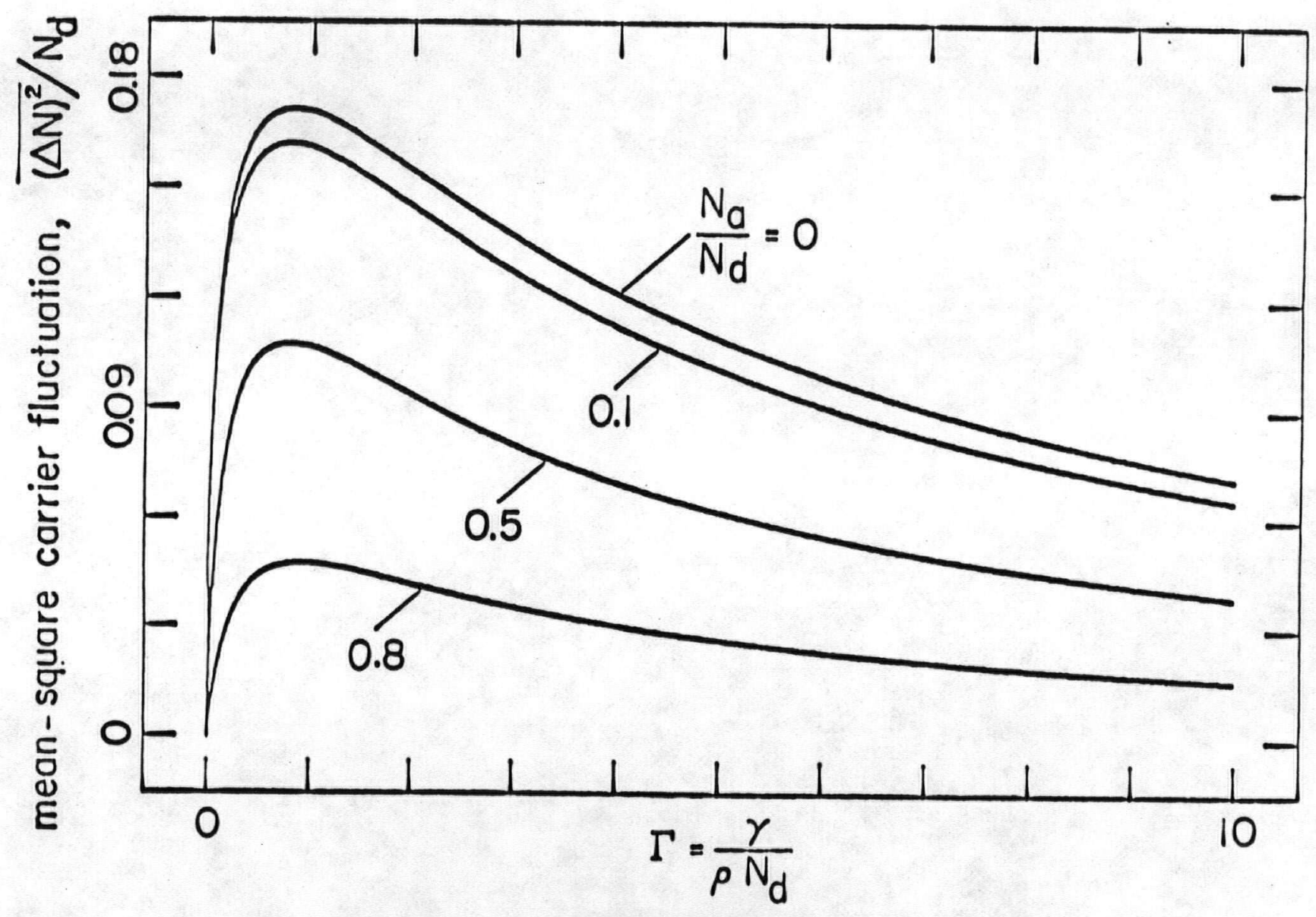

generation rate

Fig.(2.9) - Mean-square carrier fluctuation versus generation rate. 


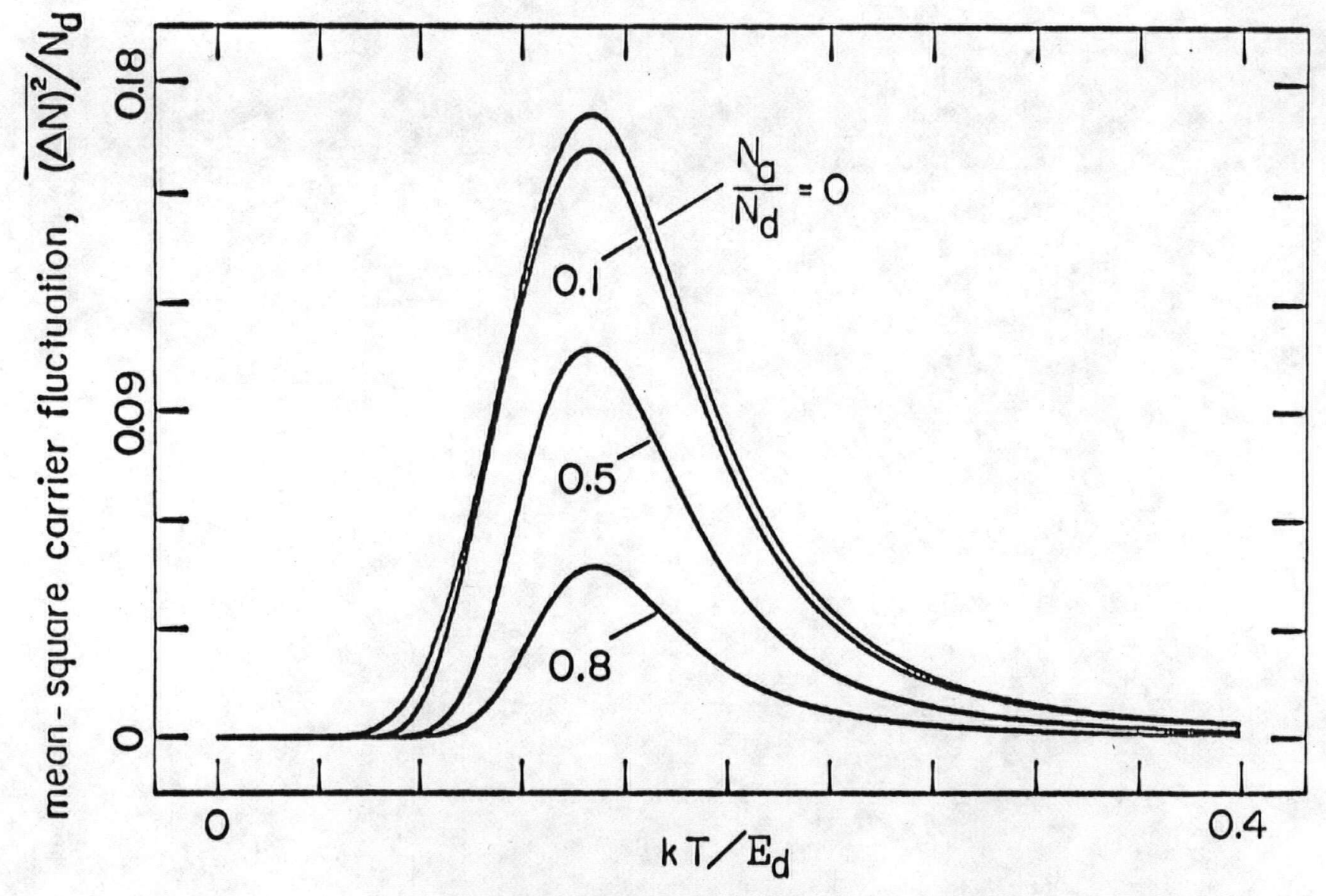

dimensionless temperature

Fig.(2.10) - Mean-square carrier fluctuation versus dimensionless temperature. 


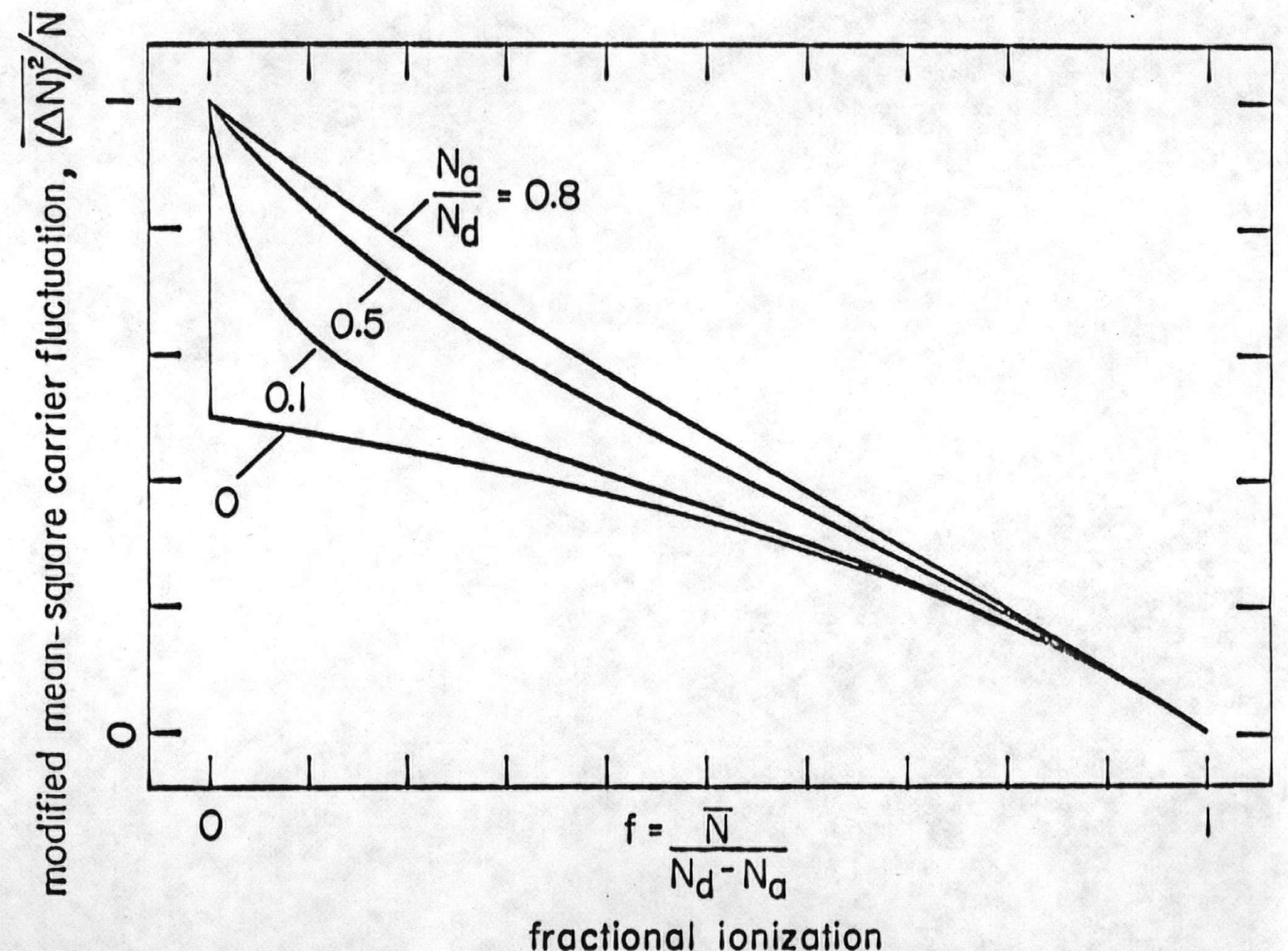

Fig.(2.11) - Modified mean-square carrier fluctuation versus fractional ionization. It represents the degree to which the statistics are Poissonian. 


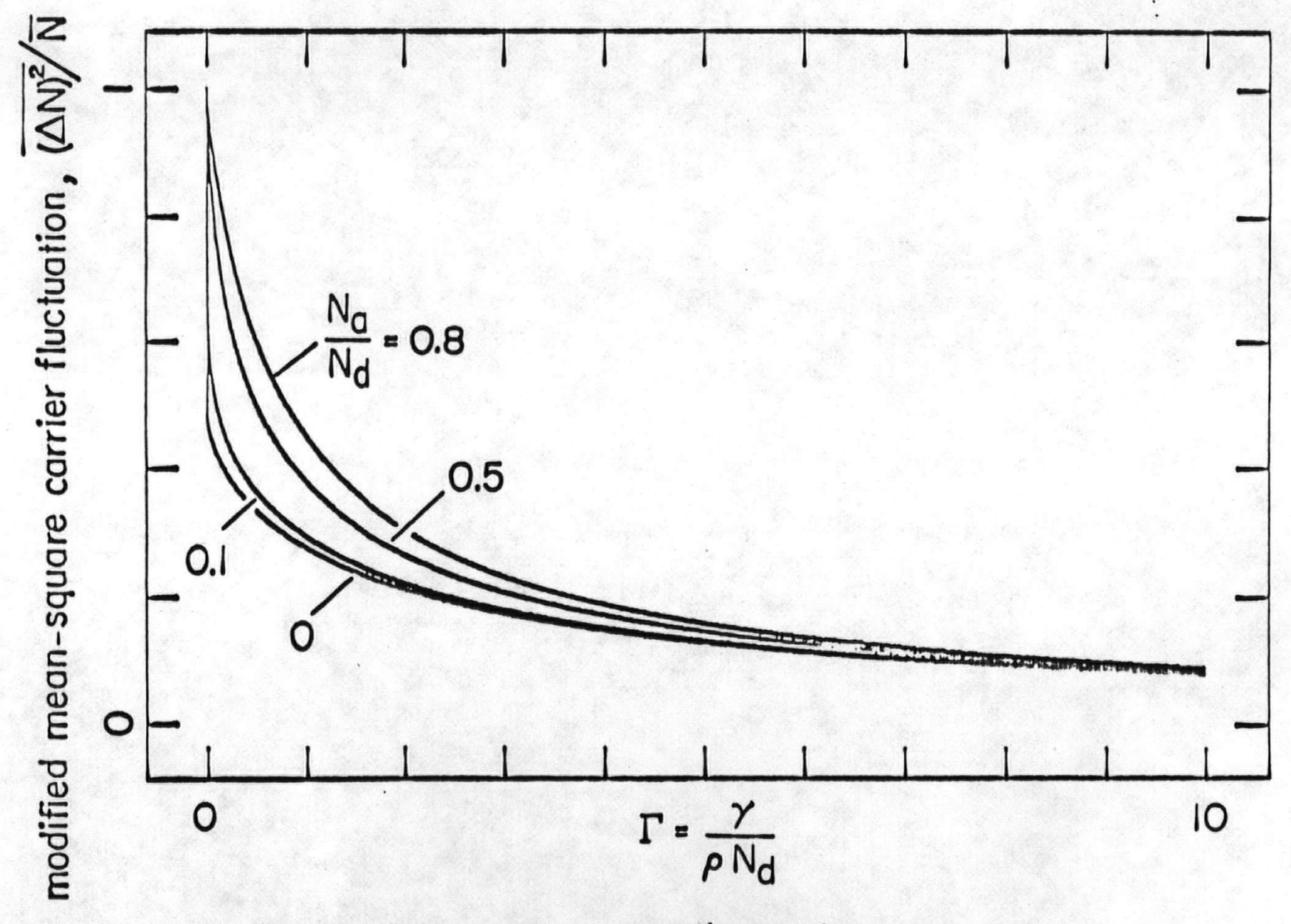

generation rate

Fig.(2.12) - Modified mean-square carrier fluctuation versus generation rate. 


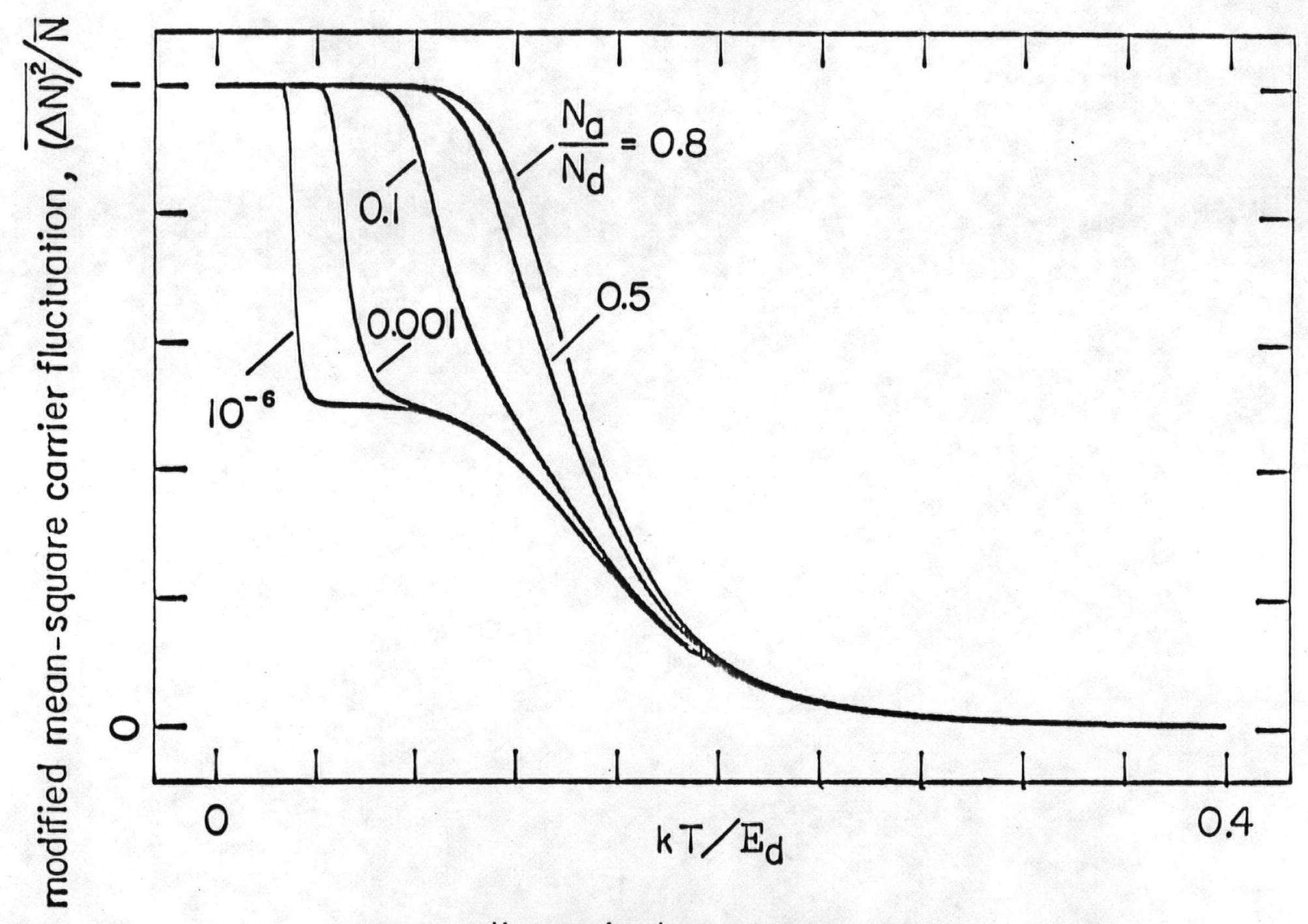

dimensionless temperature

Fig.(2.13) - Modified mean-square carrier fluctuation versus dimensionless temperature. 
(2.17), an expression for the response time can be found, after further substitution for $f$ and $\lambda$ :

$$
\tau^{\prime}=\frac{1}{\rho N_{d}} \frac{(1-f)}{f(2-f)(1-\lambda)+\lambda}
$$

Figures (2.14), (2.15), and (2.16) represent the normalized response time as a function of the same three variables $f, \Gamma$, and $k T / \varepsilon_{d}$ respectively. The normalization was necessary in order to show the response times for different compensation levels on the same plot. The mean carrier lifetime is found, by substituting equations (2.1) and (2.32) into equation (2.18), to be

$$
\bar{\tau}=\frac{1}{\rho N_{d}} \frac{1}{\lambda+f(1-\lambda)} .
$$

The mean carrier lifetime is plotted in figures (2.17), (2.18), and (2.19) as functions of $f, \Gamma$, and $k T / \varepsilon_{d}$ respectively.

The detector responsivity can be derived from the equilibrium $g-r$ condition

$$
\gamma\left(N_{d}-N_{a}-\bar{N}\right)=\rho \bar{N}\left(N_{a}+\bar{N}\right)
$$

by considering the effect on $\bar{N}$ of increasing the generation coefficient by an amount $\delta \gamma$ as follows:

$$
(\gamma+\delta \gamma)\left(N_{d}-N_{a}-\bar{N}-\delta \bar{N}\right)=\rho(\bar{N}+\delta \bar{N})\left(N_{a}+\bar{N}+\delta \bar{N}\right) .
$$

If the assumptions 


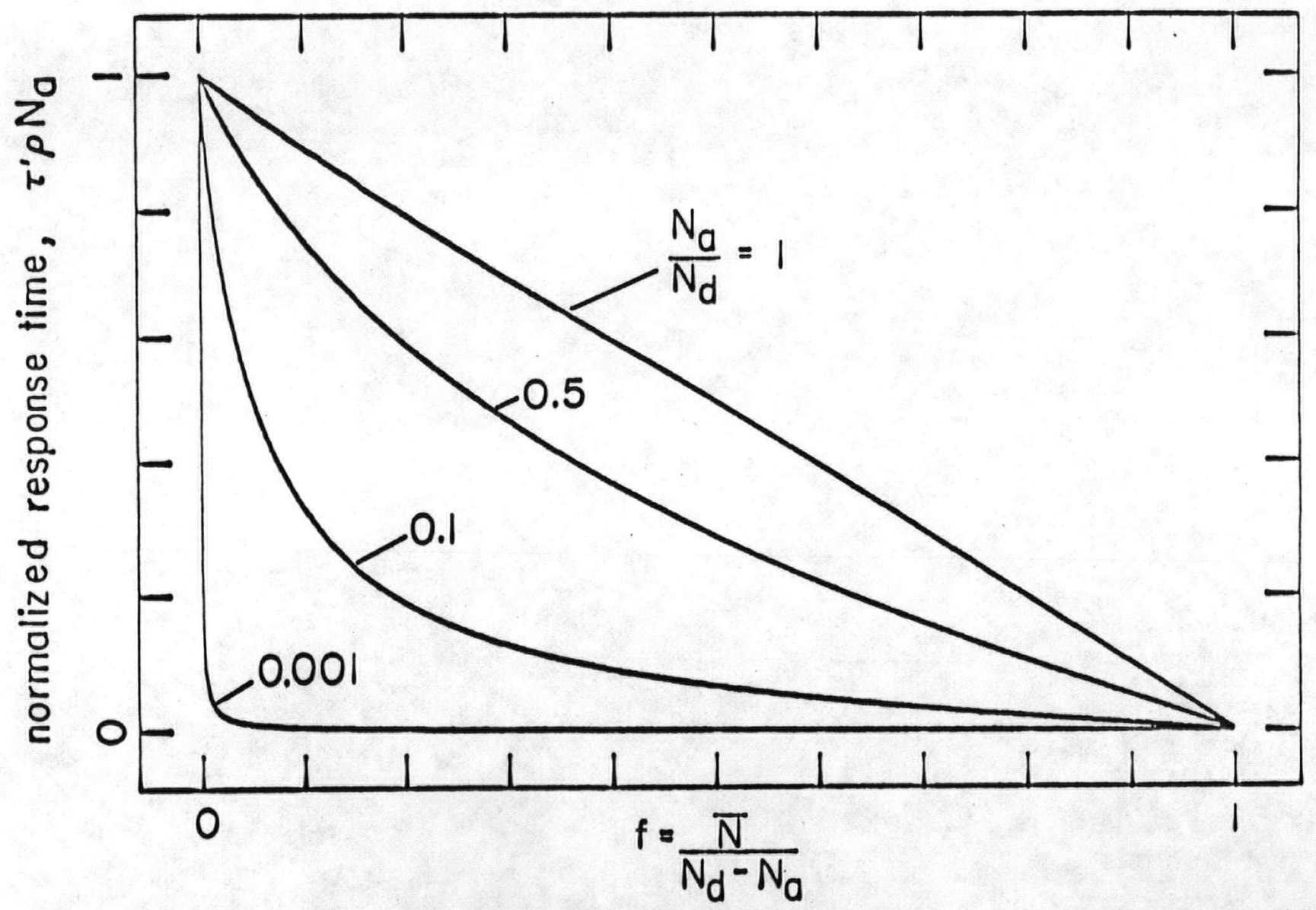

fractional ionization

Fig.(2.14) - Normalized response time versus fractional ionization. 


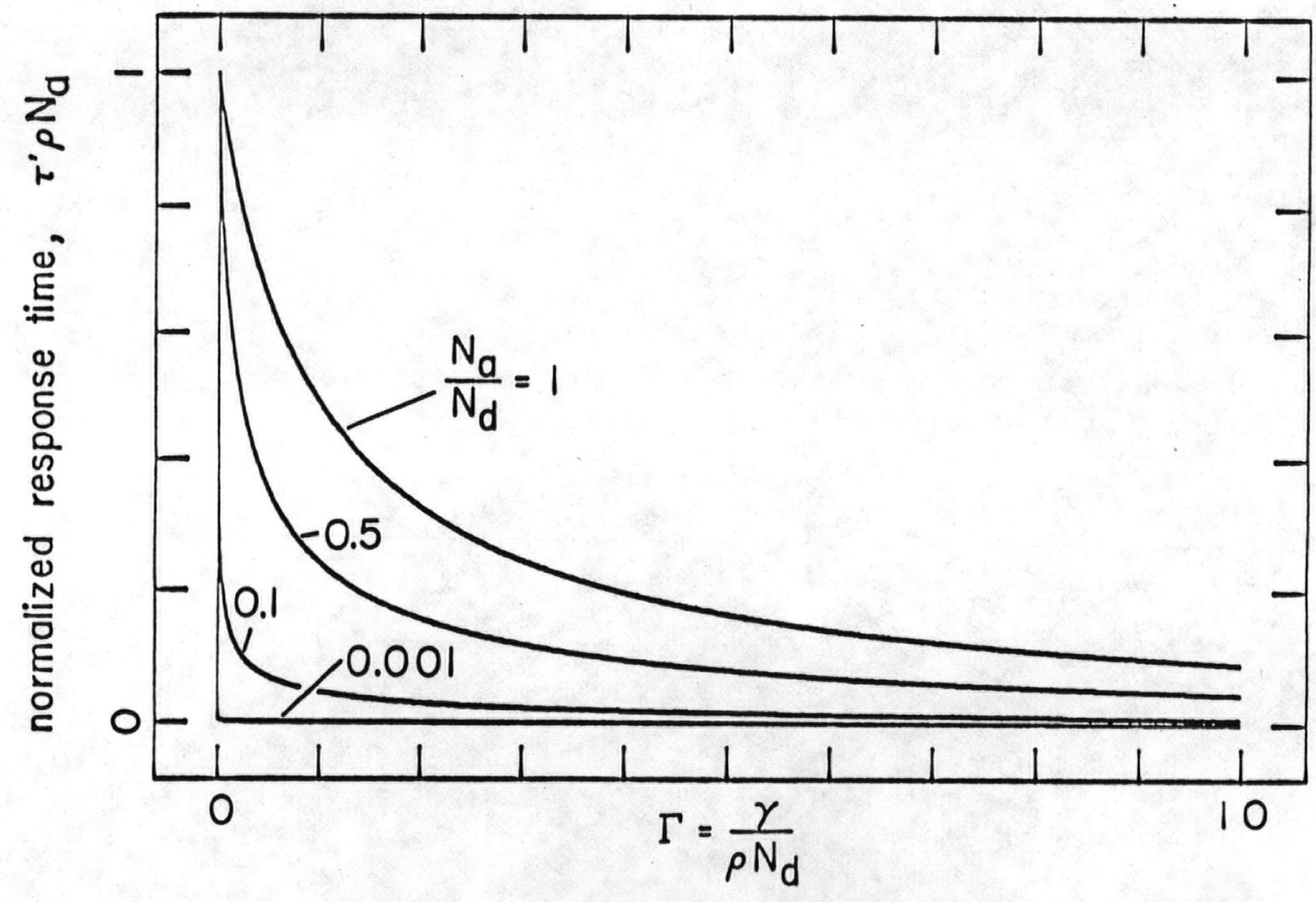

generation rate

Fig.(2.15) - Normalized response time versus generation rate. 


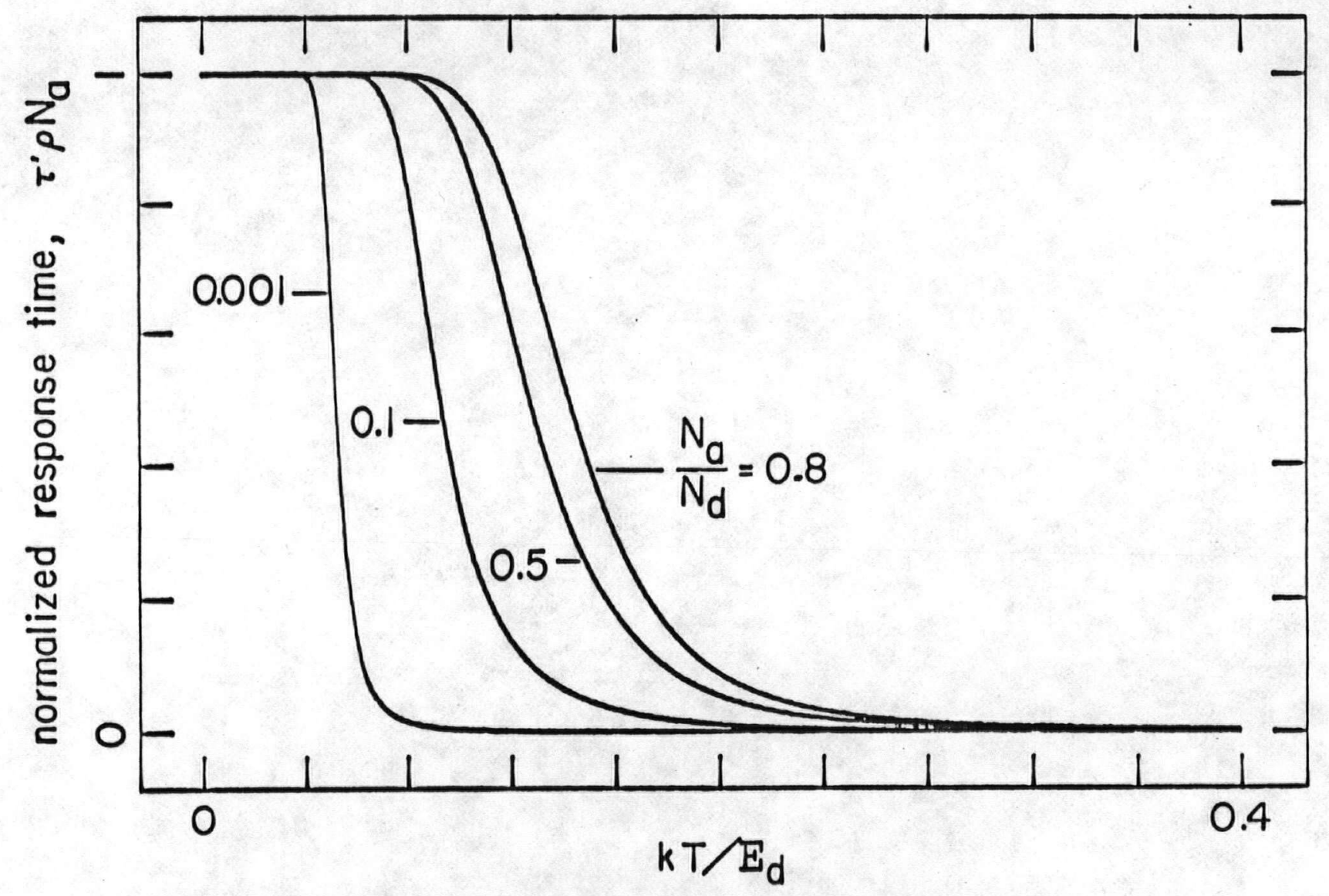

dimensionless temperature

Fig.(2.16) - Normalized response time versus dimensionless temperature. 


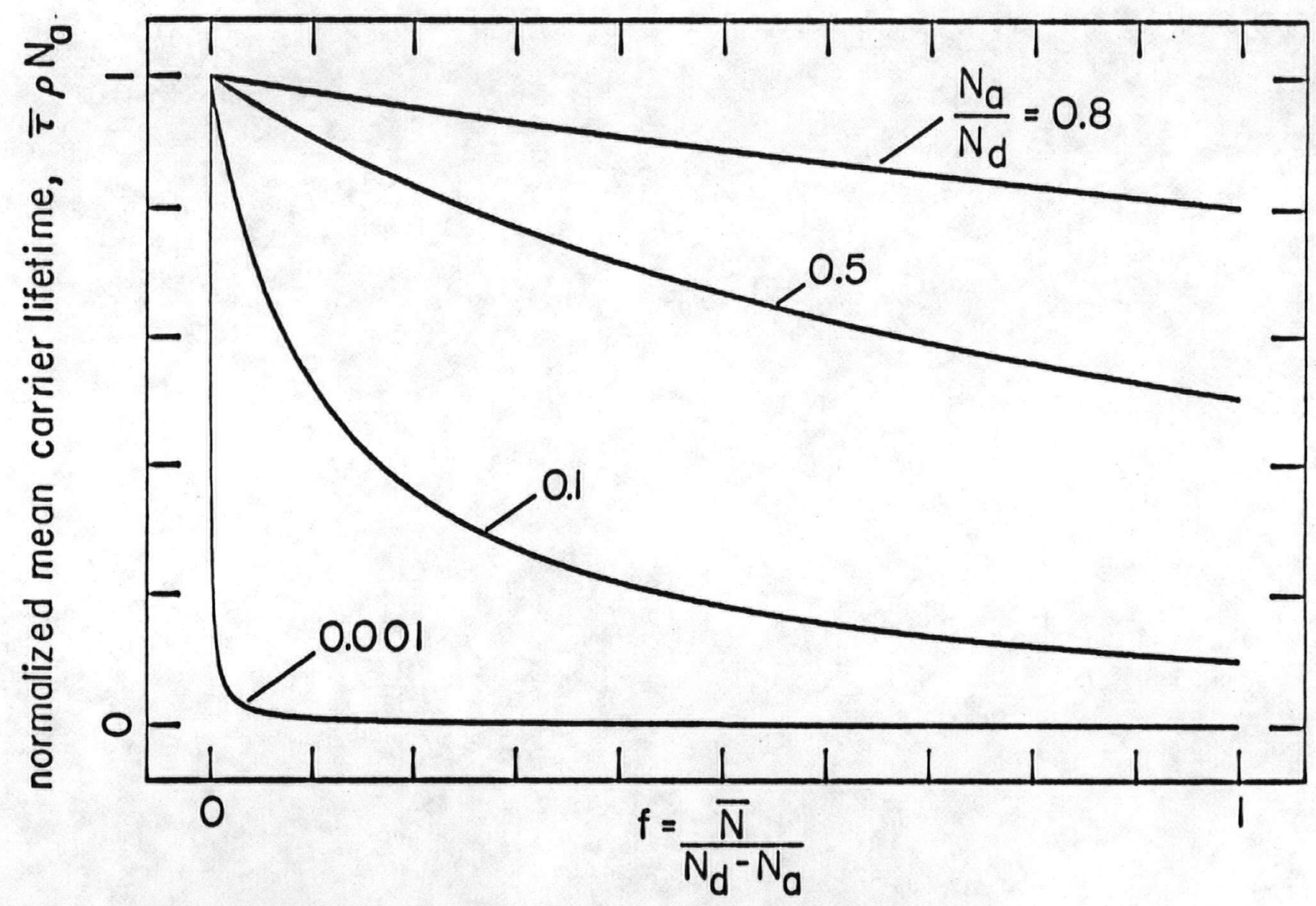

fractional ionization

Fig.(2.17) - Normalized mean carrier lifetime versus fractional ionization. 


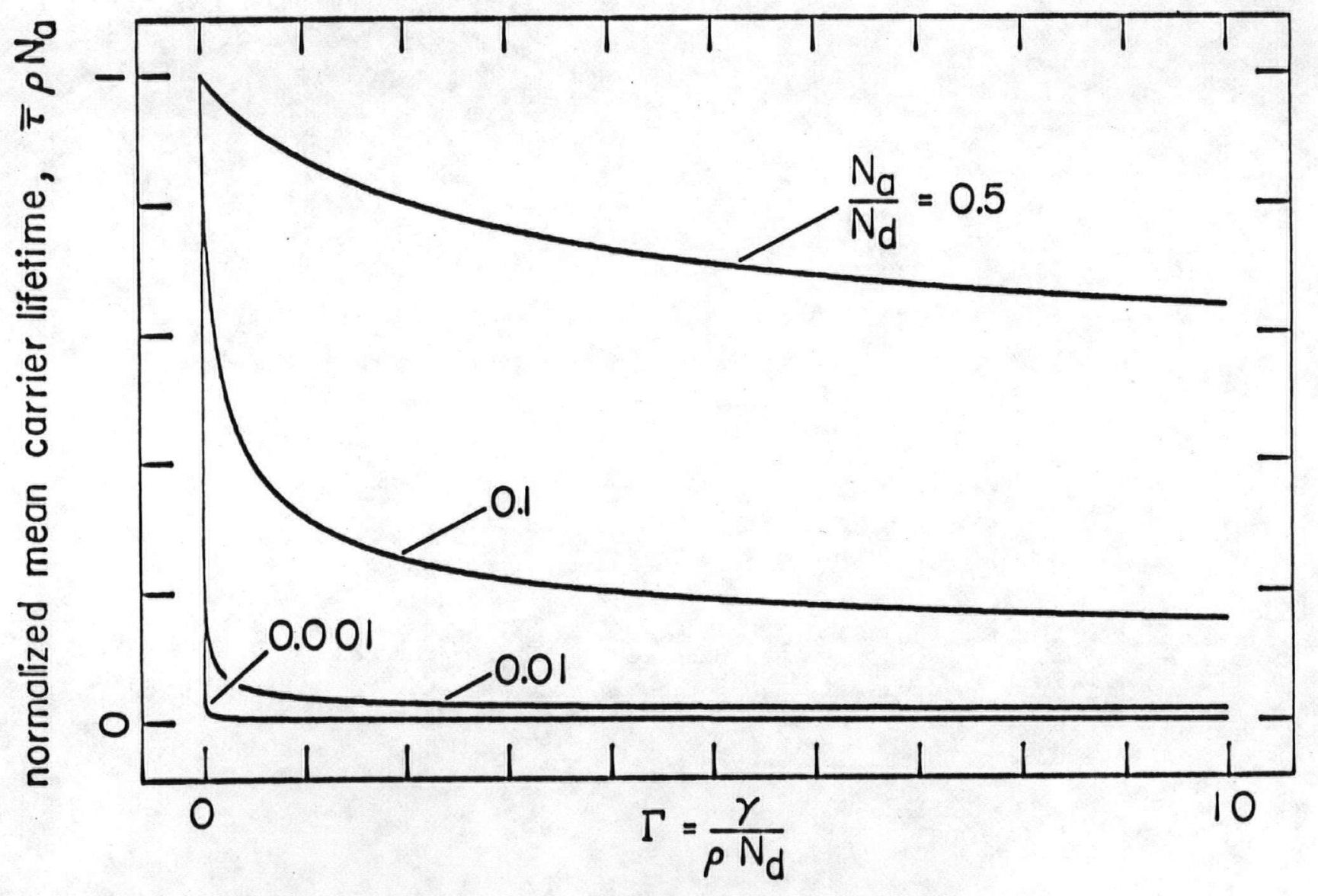

generation rate

F1g.(2.18) - Normalized mean carrier lifetime versus generation rate. 


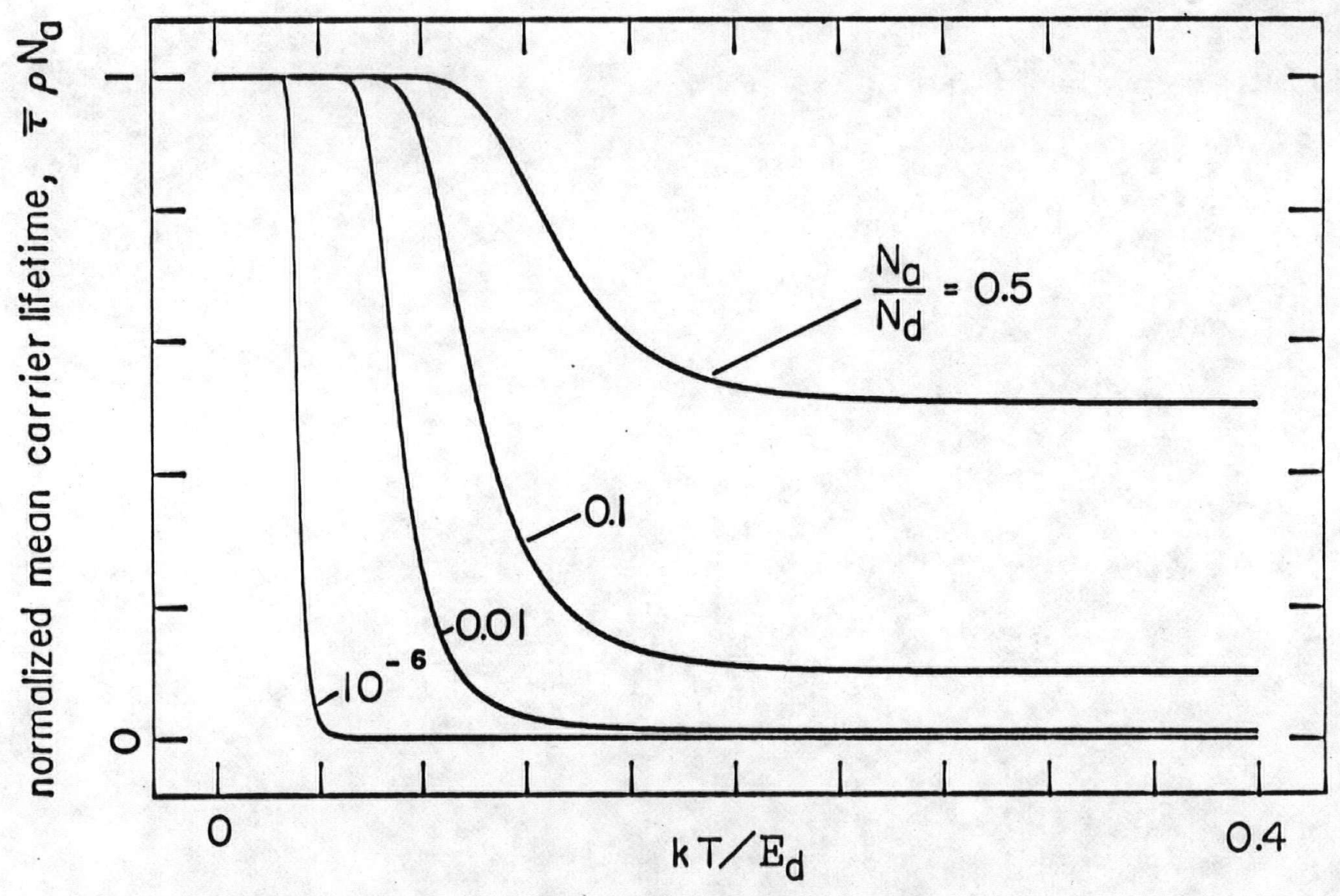

dimensionless temperature

Fig.(2.19) - Normalized mean carrier lifetime versus dimensionless temperature. 
$\delta \gamma \ll \gamma$,

$\gamma \bar{N} \ll \bar{N}$, and

$\delta \gamma \delta \bar{N} \ll \gamma \delta \bar{N}, \delta \gamma \bar{N}$

are made, then

$$
\begin{aligned}
& \delta \gamma\left(N_{d}-N_{a}-\bar{N}\right)+\gamma\left(N_{d}-N_{a}-\bar{N}\right)=\rho\left(N_{a}+\bar{N}\right) \\
& +\rho \delta \bar{N}\left(2 \bar{N}+N_{a}+\gamma / \rho\right) .
\end{aligned}
$$

Substitution of equation (2.45) into equation (2.48) leads to the expression

$$
\delta \gamma\left(N_{d}-N_{a}-\bar{N}\right)=\delta \bar{N}\left[\rho\left(2 \bar{N}+N_{a}\right)+\gamma\right] .
$$

Finally, the responsivity $R$, is given by

$$
R=\frac{\delta \bar{N}}{\delta \gamma}=\frac{N_{d}-N_{a}-\bar{N}}{\rho\left(2 \bar{N}+N_{a}\right)+\gamma},
$$

which is expressed in terms of the fractional ionization and the compensation ratio as

$$
R=\frac{1}{\rho} \frac{(1-f)^{2}(1-\lambda)}{f(2-f)(1-\lambda)+\lambda} .
$$

Figures $(2.20),(2.21)$, and (2.22) illustrate the responsivity as functions of $f, \Gamma$, and $k T / \varepsilon_{d}$ respectively. Once again, the normalization was necessary to accommodate the different compensation levels on one plot. 


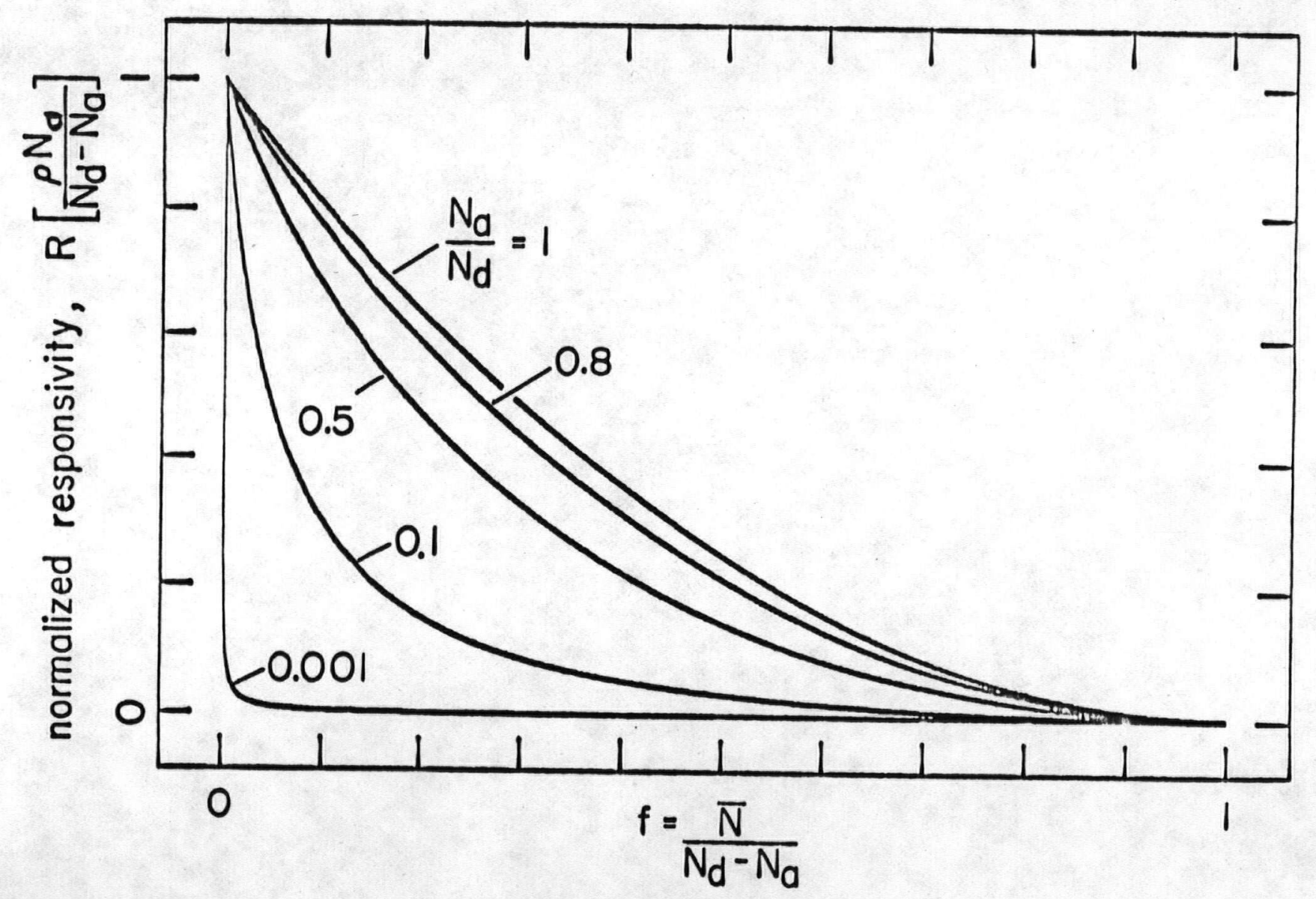

fractional ionization

Fig.(2.20) - Normalized responsivity versus fractiunal ionization. 


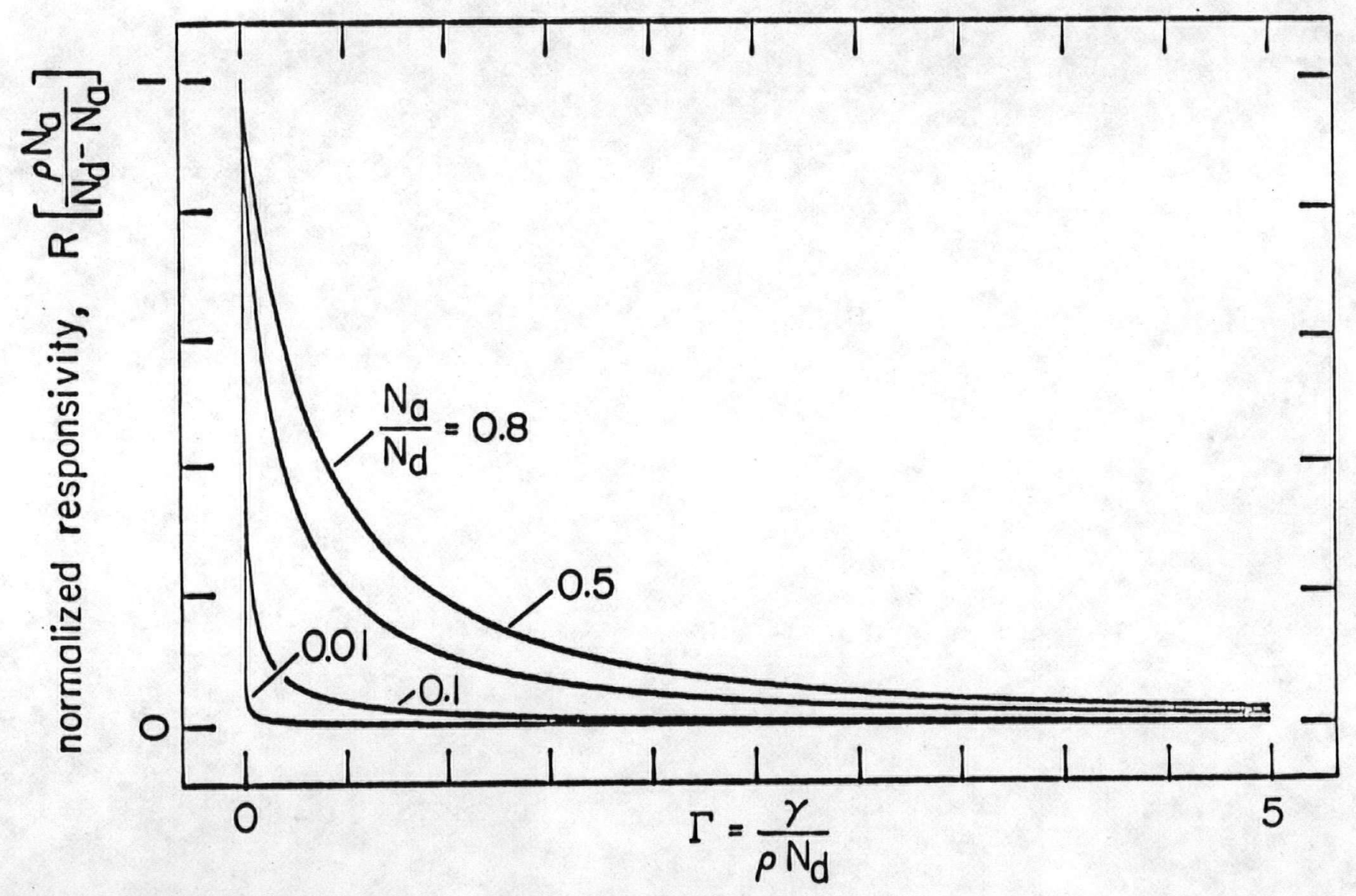

generation rate

Fig.(2.21) - Normalized responsivity versus generation rate. 


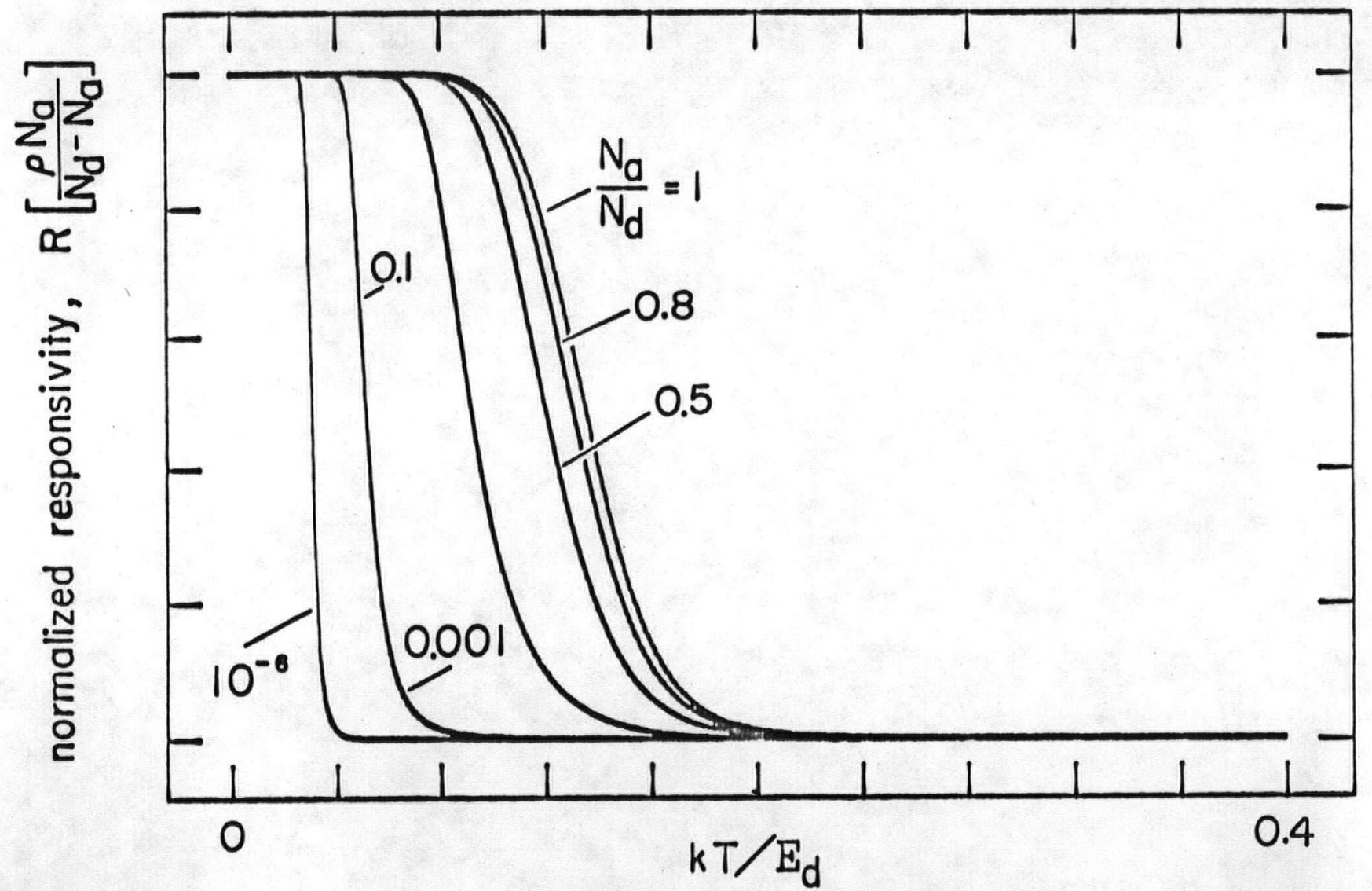

dimensionless temperature

Fig.(2.22) - Normalized responsivity versus dimensionless temperature. 
The noise equivalent power, or NEP, of a given detector is defined as the value of the signal power for which the signal-tonoise ratio is equal to unity. It is derived as follows: The generation coefficient of equation (2.1) can be expressed as

$$
\gamma=\gamma_{t h}+\frac{\eta}{h v} \frac{\left(P_{B}+P_{S}\right)}{\left(N_{d}-N_{a}\right)}=\gamma_{t h}+\gamma_{B}+\gamma_{S},
$$

where $\gamma_{t h}$ is the thermal contribution. $P_{B}$ and $P_{S}$ are the background and signal powers respectively. $\gamma_{B}$ and $\gamma_{S}$ represent the background and signal contributions to the generation coefficient respectively . $\eta$ is defined as the quantum efficiency, $h$ is Planck's constant, and $v$ is the optical frequency.

An incremental change of $P_{S}$ in the total power $P$ will lead to a change in the generation rate $\gamma$ of $\delta \gamma$ and perturb $\bar{N}$ by an amount $\delta \bar{N}$, which gives

$$
(\gamma+\delta \gamma)\left(N_{d}-N_{a}-\bar{N}-\delta \bar{N}\right)=\rho(\bar{N}+\delta \bar{N})\left(N_{a}+\bar{N}+\delta \bar{N}\right)
$$

Combining terms, using the assumptions of equation (2.47), and solving for the change in $\bar{N}$ of $\delta \bar{N}$ results in

$$
\delta \bar{N}=\frac{\delta \gamma_{j}\left(N_{d}-N_{a}-\bar{N}\right)}{\rho\left(N_{a}+2 \bar{N}\right)+\gamma} .
$$

Solving for $\gamma / \rho$ when equation (2.1) is set equal to equation (2.2) yields 


$$
\frac{\gamma}{\rho}=N_{d} \frac{f[\lambda+f(1-\lambda)]}{(1-f)},
$$

and upon substituting into equation (2.54) results in

$$
\delta \bar{N}=\frac{\delta \gamma}{\rho} \frac{(1-\lambda)(1-f)^{2}}{f(2-f)(1-\lambda)+\lambda}
$$

The signal-to-noise ratio depends on the signal current and the noise current according to 12

$$
\left(\frac{S}{N}\right)^{2}=\frac{\bar{i}_{s}^{2}}{\bar{i}_{g r}^{2}}
$$

The mean signal current is given by equation (2.20) as

$$
\bar{i}=\frac{e V}{L^{2}} \mu_{n} \delta \bar{N} .
$$

The mean-square $g-r$ current noise of equation (2.31) can be expressed in terms of $f$ and $\lambda$ as

$$
\overline{i_{g r}^{2}}=\frac{4}{\rho}\left(\frac{e V \mu_{n}}{L^{2}}\right)^{2} \frac{f(1-\lambda)(1-f)^{2}[\lambda+f(1-\lambda)] \Delta f}{[f(2-f)(1-\lambda)+\lambda]^{2}} .
$$

Substitution into equation (2.57) yields

$$
\left(\frac{S}{N}\right)^{2}=\frac{(\delta \bar{N})^{2}}{4} \rho \frac{[f(2-f)(1-\lambda)+\lambda]^{2}}{f(1-\lambda)(1-f)^{2}[\lambda+f(1-\lambda)] \Delta f} .
$$

Defining $\delta \gamma$ in equation (2.56) to have the form

$$
\delta \gamma=\frac{\eta P_{S}}{h v\left(N_{d}-N_{a}\right)},
$$


and substituting for $(\delta \bar{N})^{2}$ in equation $(2.60)$ results in

$$
\left(\frac{S}{N}\right)^{2}=\frac{1}{4 \rho \Delta f}\left(\frac{\eta}{h v}\right)^{2} \frac{P_{S}^{2}}{\left(N_{d}-N_{a}\right)^{2}} \frac{(1-\lambda)(1-f)^{2}}{f[\lambda+f(1-\lambda)]} .
$$

The noise equivalent power, given by setting equation (2.62) equal to unity, is

$$
\text { NEP }=\frac{h v}{\eta} \sqrt{4 \rho \Delta f}\left(N_{d}-N_{a}\right) \frac{[f(1-\lambda)[\lambda+f(1-\lambda)]]^{\frac{1}{2}}}{(1-\lambda)(1-f)} .
$$

The NEP is plotted as functions of $f, \Gamma$, and $k T / \varepsilon_{d}$ in figures (2.23), (2.24), and (2.25) respectively.

One will note that in figure (2.24) the lower curve represents the NEP of an ideal photoconductive detector. The ideal NEP in figure (2.24) was calculated for zero compensation and should only be compared to the theoretical NEP for zero compensation. The NEP of the extrinsic photoconductive detector is known to be greater than that of the ideal photon detector by a factor of at least the square root of two. This results from the fact that the fluctuations in the generation and recombination rates are comprised of two statistically independent noise sources compared to the ideal photon detector which is limited only by photon noise. The NEP of the ideal photoconductive detector is given by ${ }^{13}$

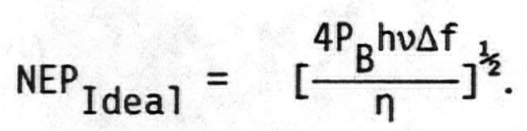




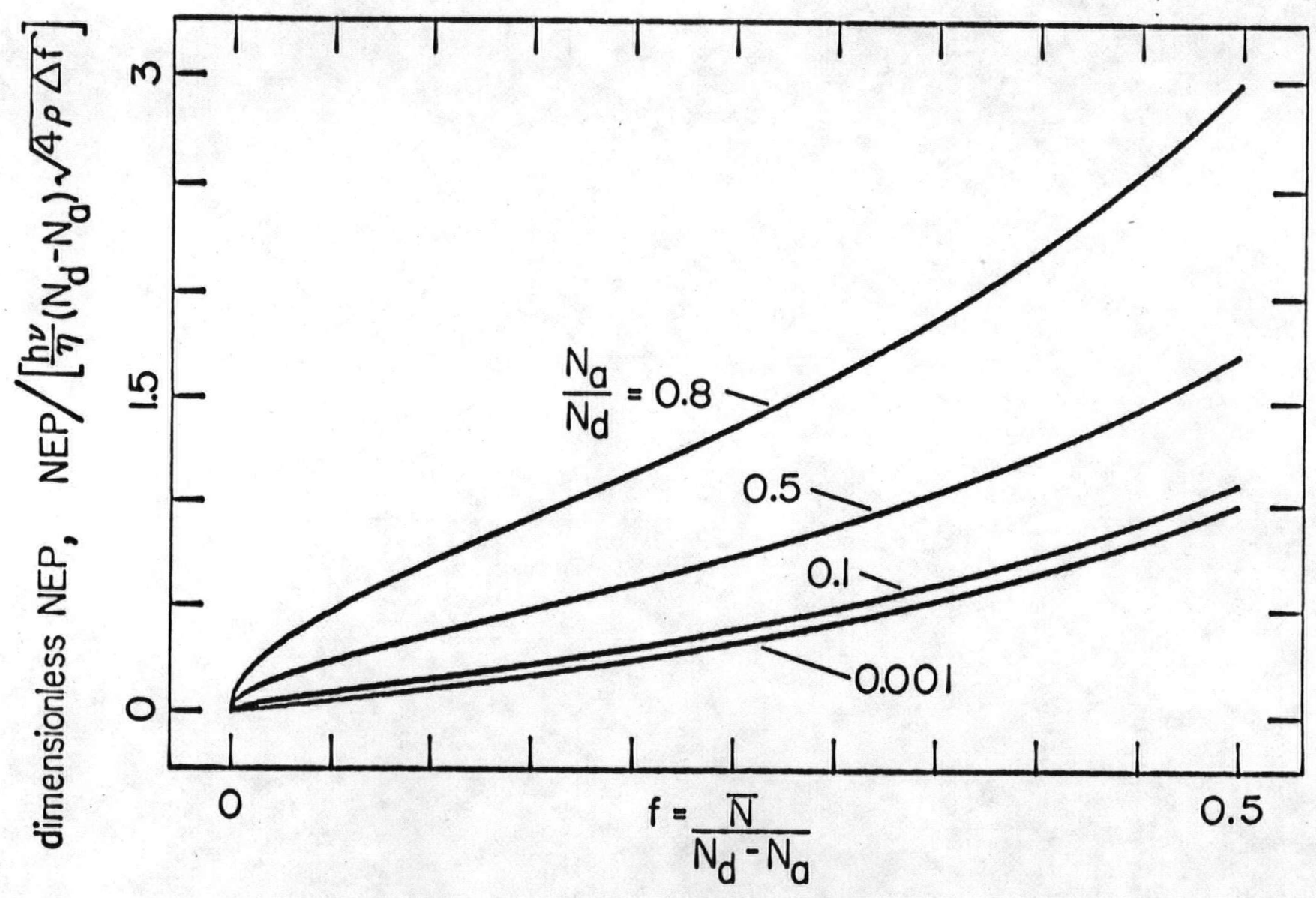

fractional ionization

Fig.(2.23) - Dimensionless NEP versus fractional ionization. 


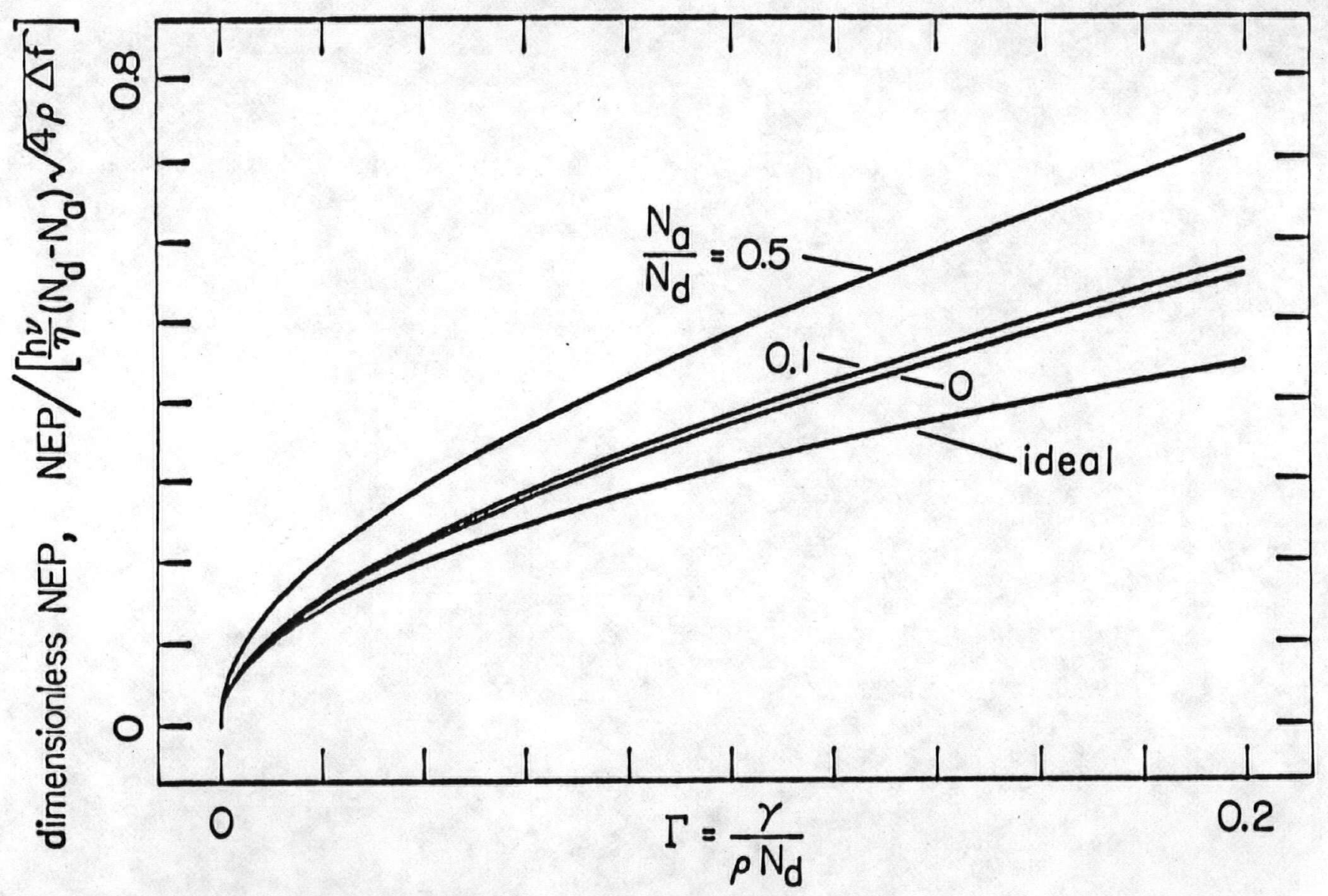

generation rate

Fig.(2.24) - Dimensionless NEP versus generation rate. 


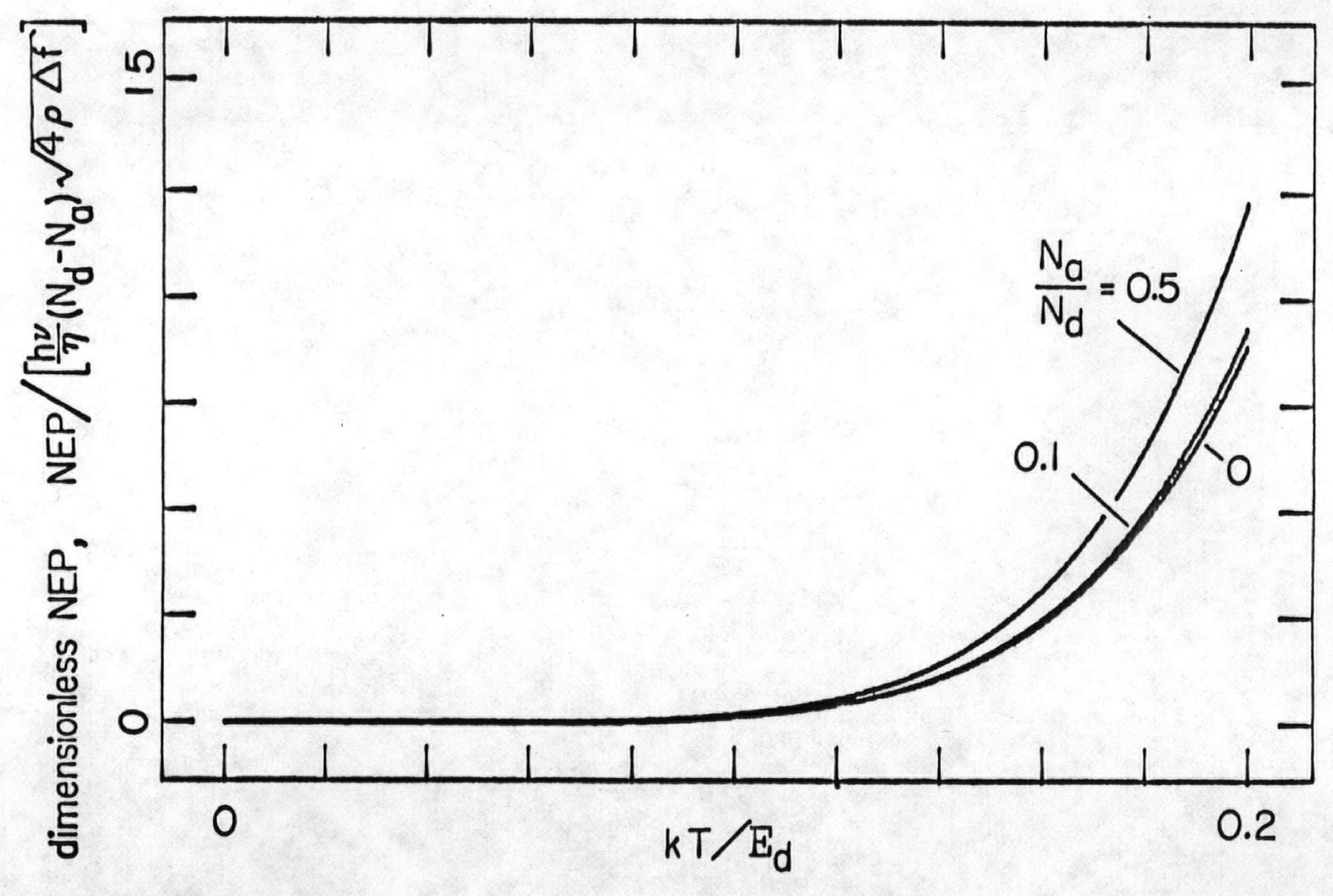

dimensionless temperature

Fig.(2.25) - Dimensionless NEP versus dimensionless temperature. 
In the theoretical calculation of the NEP given above, the generation coefficient due to the background was taken to be

$$
\gamma_{B}=\frac{\eta P_{B}}{h v\left(N_{d}-N_{a}\right)}
$$

Equation (2.64) for the ideal NEP can be expressed equivalently as

$$
\mathrm{NEP}_{\text {Ideal }}=\left[\frac{\mathrm{hv}}{\eta} \sqrt{4 \rho \Delta \mathrm{f}} \operatorname{sed} \sqrt{N_{d}\left(N_{d}-N_{a}\right)}\right] \sqrt{\mathrm{T}} .
$$

Substituting equations (2.55) and (2.65) into equation (2.64) results in an ideal NEP given by

$\mathrm{NEP}_{\text {Ideal }}=\frac{h v}{\eta} \sqrt{4 \rho \Delta f} \sqrt{N_{d}\left(N_{d}-N_{a}\right)} \quad\left[\frac{f[\lambda+f(1-\lambda)]}{(1-f)}\right]^{\frac{1}{2}}$.

Thus, the ratio of the theoretical NEP for the impurity compensated extrinsic photoconductive detector to the NEP of the ideal photoconductive detector is

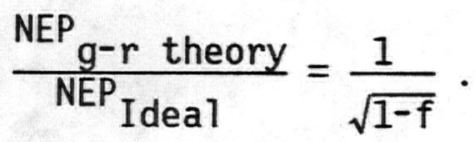

\subsection{Conclusion}

This chapter presented a complete theory of the generationrecombination noise associated with extrinsic photoconductive detectors. The theory accounts for the effect of impurity compensation on detector properties and stresses that this effect is often far from negligible. 
The treatment of NEP allows for the calculation of the magnitude of the recombination coefficient $\rho$, from an empirical fit of one's data to the plots provided. Insight as to the functional form of this coefficient may be gained from such analyses.

It is hoped that this theory will provide a means for improved characterization of this class of detectors. We also hope that this theory will lead to improved photoconductive materials by providing the detector manufacturer with insight to better design criteria. 


\section{REFERENCES}

1. Reference 2, Chapter 1.

2. Reference 1, Chapter 1.

3. R.E. Burgess, "The Statistics of Charge Carrier Fluctuations in Semiconductors," (Proceedings of the Physical Society of London, B 69,1955$)$.

4. Reference 2, Chapter 1 .

5. Reference 3.

6. R.W. Boyd, Radiometry and the Detection of Optical Radiation, (Wi ley-Interscience, New York, 1983) pp. 169-176.

7. Reference 6, page 172.

8. Reference 3.

9. Reference 6, page 170.

10. Reference 6, page 176.

11. R.A. Smith, The Detection and Measurement of Infrared Radiation, (Oxford University, London, 1968).

12. Reference 6, page 124.

13. Reference 6, page 135.

14. E.S. Yang, Fundamentals of Semiconductor Devices, (McGraw-Hill, New York, 1978). 
CHAPTER 3

EXPERIMENTAL SETUP OF A Ge:Ga DETECTION SYSTEM

\subsection{Introduction}

The aim of this chapter is to present a detailed description of the constituent components of the 100 micron detection system used in the experimental phase of the author's research. Figure (3.1) illustrates the experimental setup of the Ge:Ga detection system.

The system was used to take responsivity and noise data, first as a function of the detector temperature and secondly as a function of photon flux from a blackbody source. In chapter 4, a comparison is made between the experimental and theoretical results and the initial tests leading to system optimization are reviewed.

\subsection{The Ge:Ga Detector}

The Ge:Ga extrinsic photoconductive detectors used in this research were given to the author by Don Langford of Jet Propulsion 


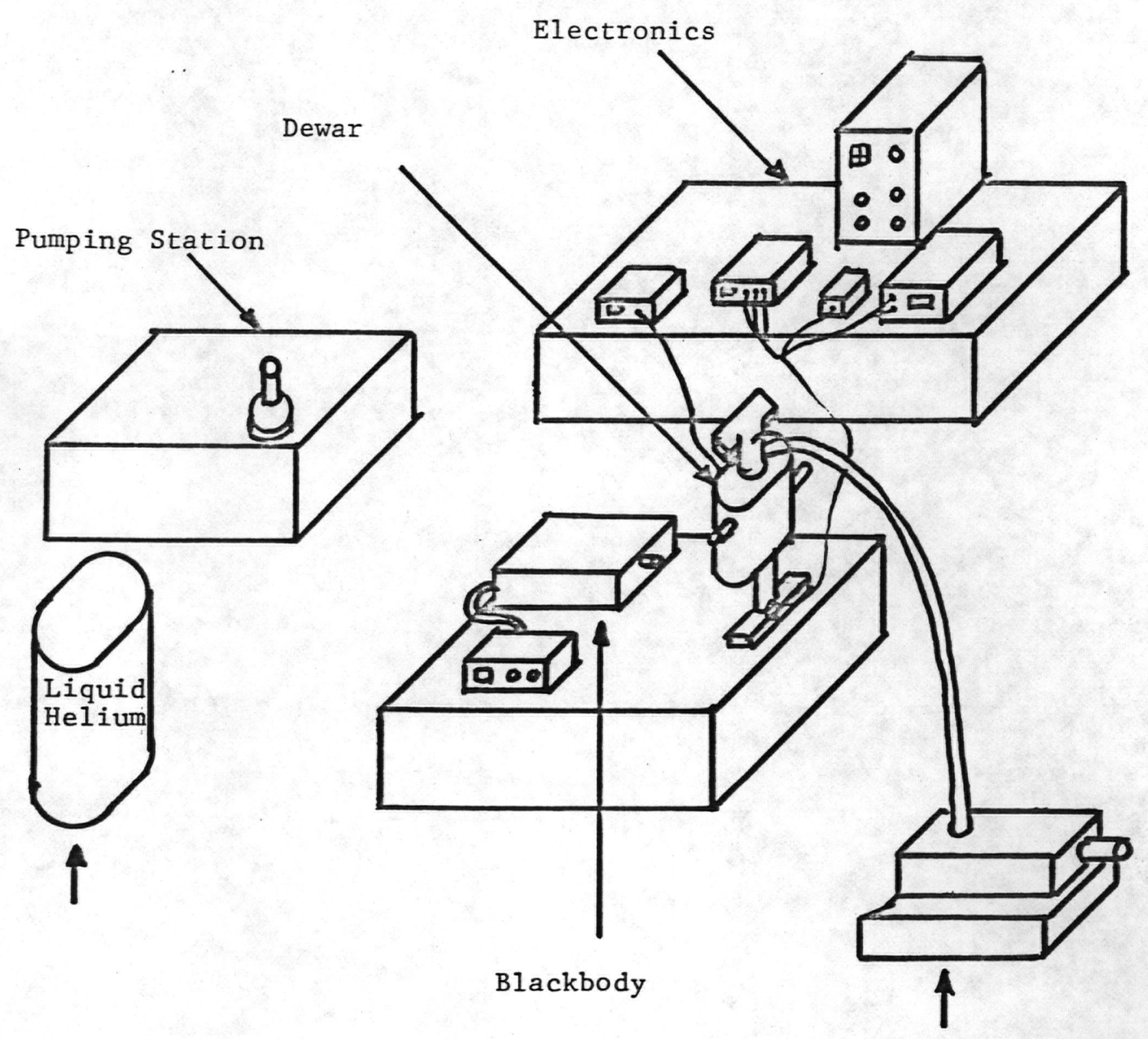

Helium Resevoir Pump

Fig.(3.1) - Experimental Setup 
Laboratory. They are the type that were used in the Infrared Astronomy Satellite (IRAS) focal plane. The peak response of these detectors occurs at a wavelength of 101 microns as shown by the response curve of figure (3.2). ${ }^{1}$

The energy band diagram shown in figure (3.3) illustrates the impurity levels in the Ge:Ga detectors. The germanium was doped with gallium to a concentration of approximately $2.5 \times 10^{14} \mathrm{~cm}^{-3}$. The compensating or residual donor concentration before doping was not known, but was assumed to be less than $5 \times 10^{11} \mathrm{~cm}^{-3} .2$ The detector measures only $1.5 \times 1.5 \times 1.4 \mathrm{~mm}^{3}$, which made soldering it to its pedestal a difficult task. The electrical contacts were made by boron ion implant and metallization by evaporation of chromium and then gold.

The detector mount and integrating cavity are illustrated in figure (3.4). One electrode of the detector is soldered to a pedestal on the mount using low temperature indium solder. A hair-thin gold wire is soldered to the remaining electrode and is connected to the signal lead. A $1 \mathrm{~mm}$ diameter hole was drilled into the side of the integrating cavity which fits over the detector. The purpose of the integrating cavity is to increase the responsivity of the detector by reflecting light for multiple passes through the detector. This results in a quantum efficiency approaching unity. The detector is oriented at $45^{\circ}$ with respect to the line of sight to eliminate fresnel reflections and to reduce uneven illumination. ${ }^{3}$ 


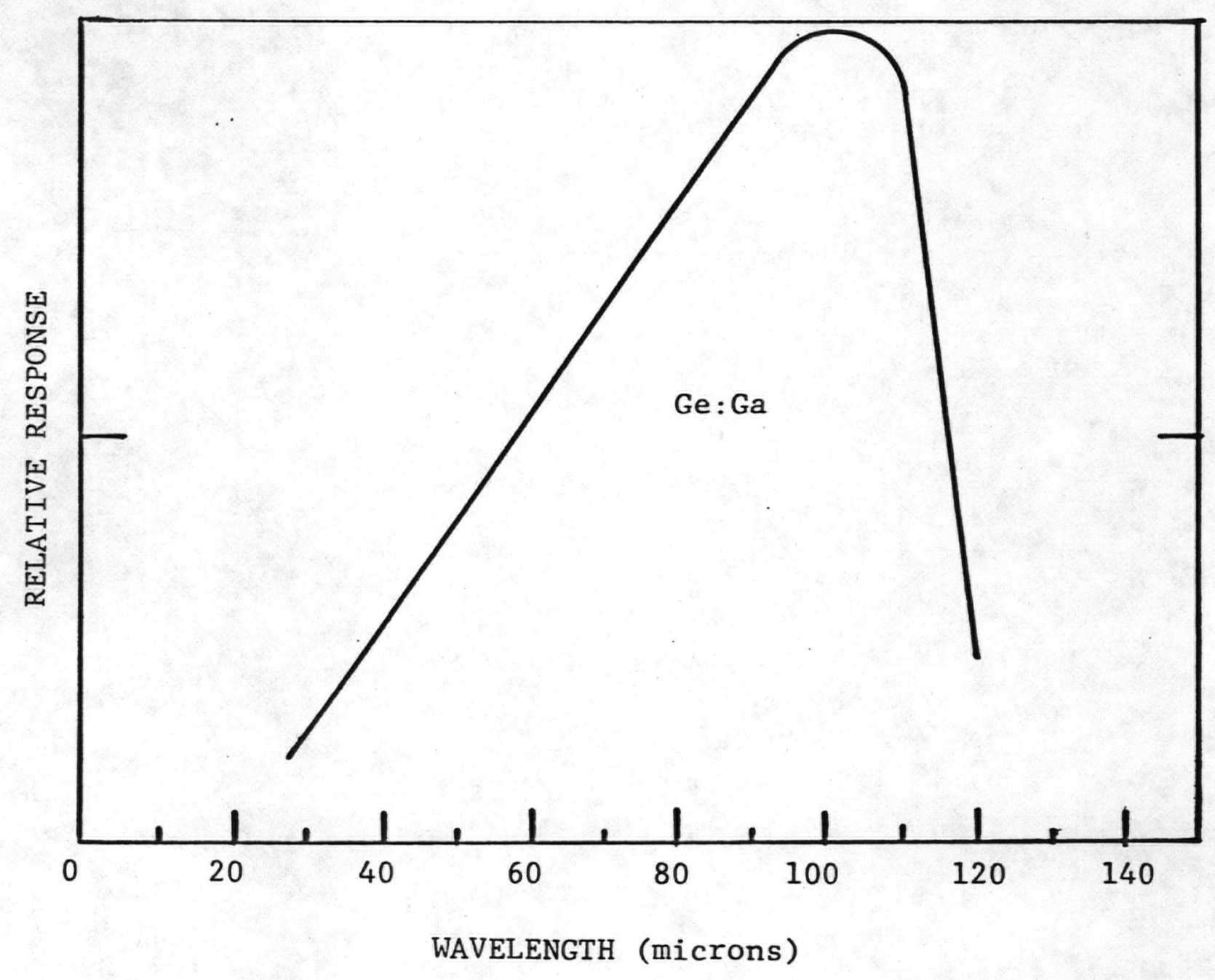

Fig(3.2) - Relative Wavelength Response of Ge:Ga Detector 


\section{IMPURITY LEVELS IN GE:GA AT $2.5^{\circ} \mathrm{K}$}
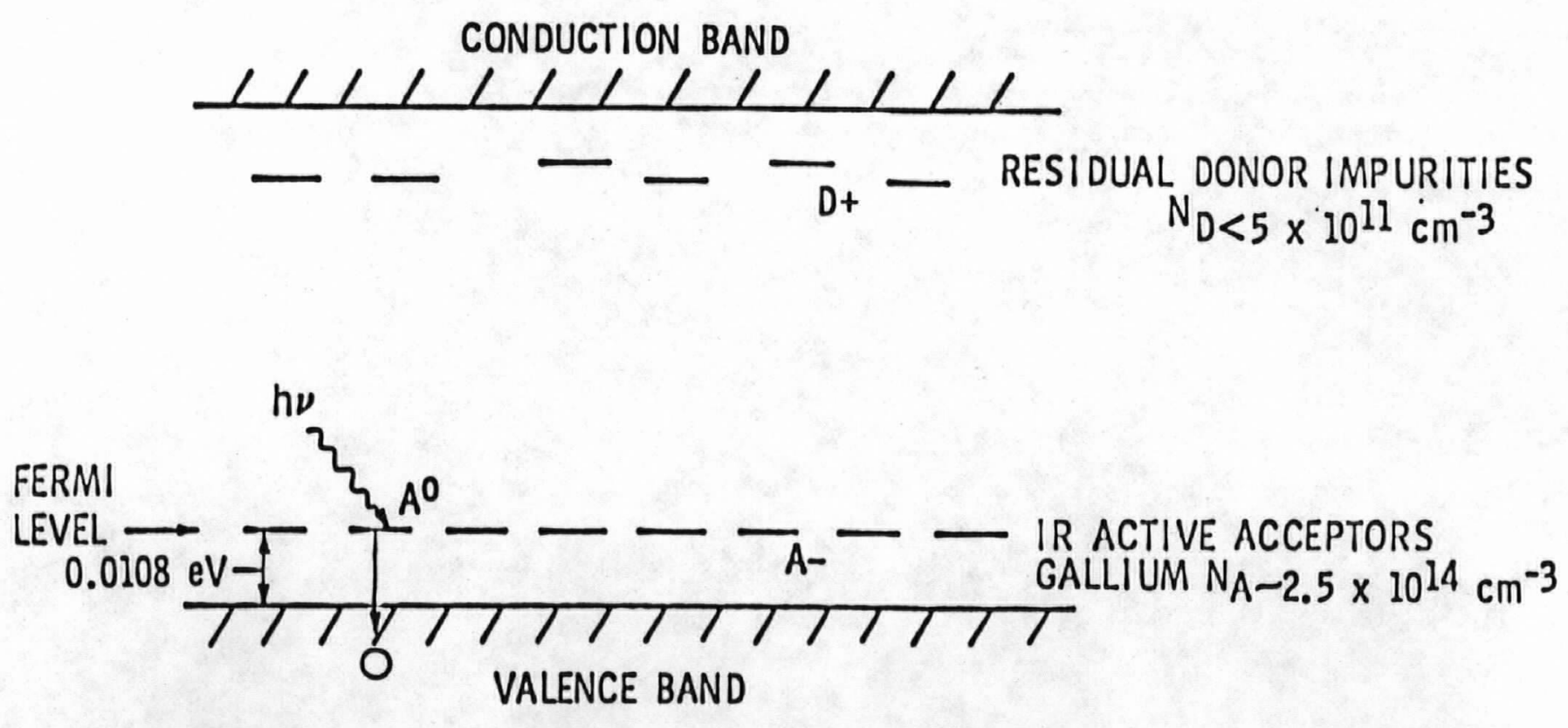

Fig.(3.3) - Impurity levels in Ge:Ga at 2.5 degrees Kelvin. 

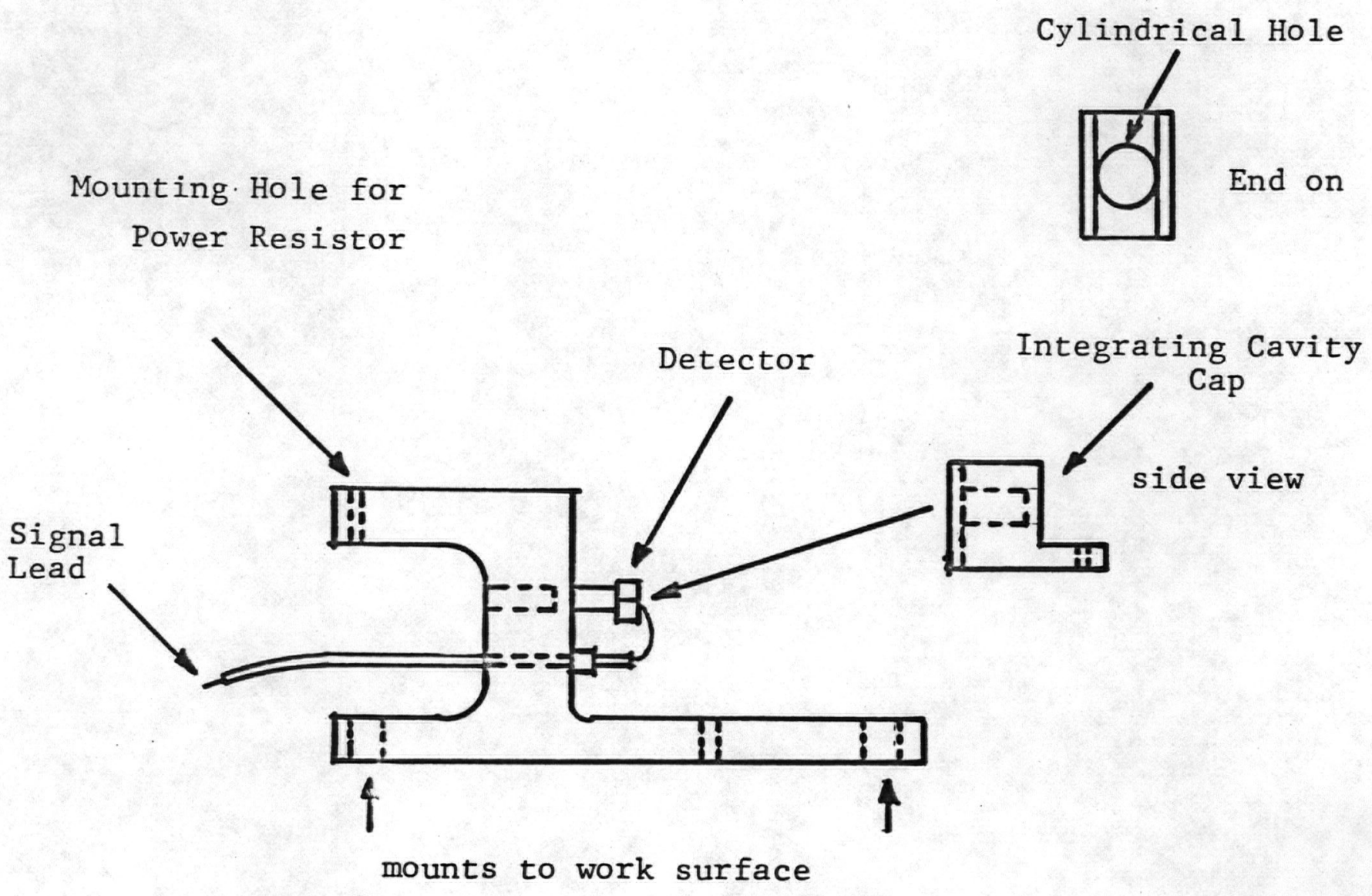

Fig(3.4) - DETECTOR MOUNT AND INTEGRATING CAVITY 


\section{3 Helium Dewar}

The dewar shown in figure (3.5) is a modified version of a model HLDT-1 Helium Dewar made by Hofman Laboratories Inc., circa 1965. The primary alteration involved extending the work surface by approximately 7 inches to the level of the windows. This configuration solved the problems of two previously considered designs. The first design considered was to leave the work surface where it was, several inches from the window, and employ cooled reflective optics to project radiation down the inside of the cold shield. This presented two problems, however; alignment and a restricted field of view. The second design that was given some consideration involved the mounting of a 7 inch copper rod to the old work surface (at the base of the cold shield), forming a new work surface at the window level. This eliminated the need for reflective optics, but did pose another problem; the thermal resistance was quite appreciable and temperatures below $17^{\circ}$ Kelvin could not be reached. Extension of the helium reservoir to the window level solved both of these design problems, resulting in a direct line of sight to the detector through the window and permitting temperatures approaching $4.2^{\circ}$ Kelvin to be reached (temperatures as low as $2^{\circ}$ Kelvin could be reached by pumping on the helium reservoir).

A gold-plated cold shield maintained at a temperature approaching $77^{\circ}$ Kelvin by liquid nitrogen surrounds the extended helium reservoir tube and work surface on which the detector holder is mounted as illustrated in figure (3.6). The cold shield also serves 


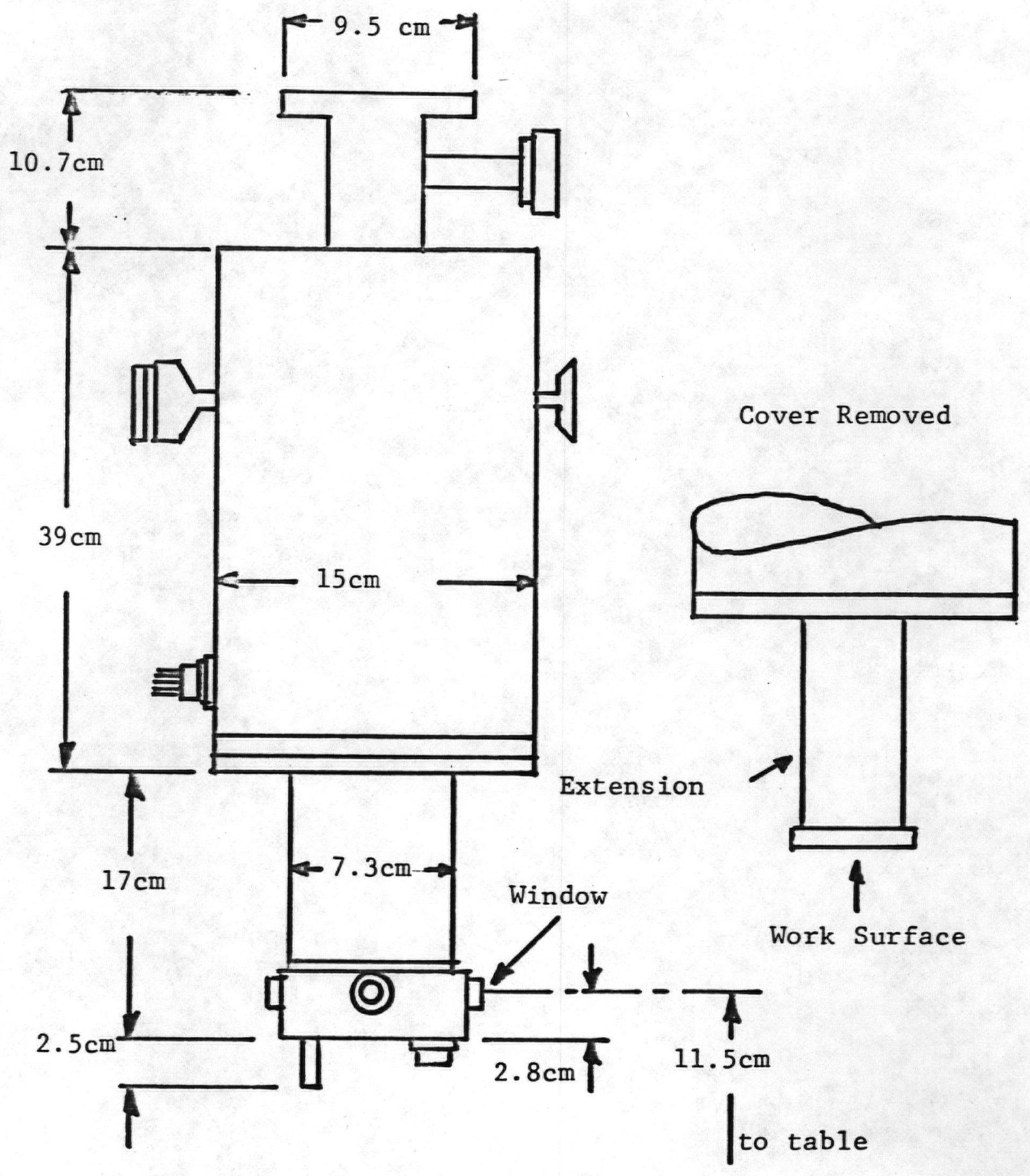

Fig.(3.5) - HELIUM DEWAR 


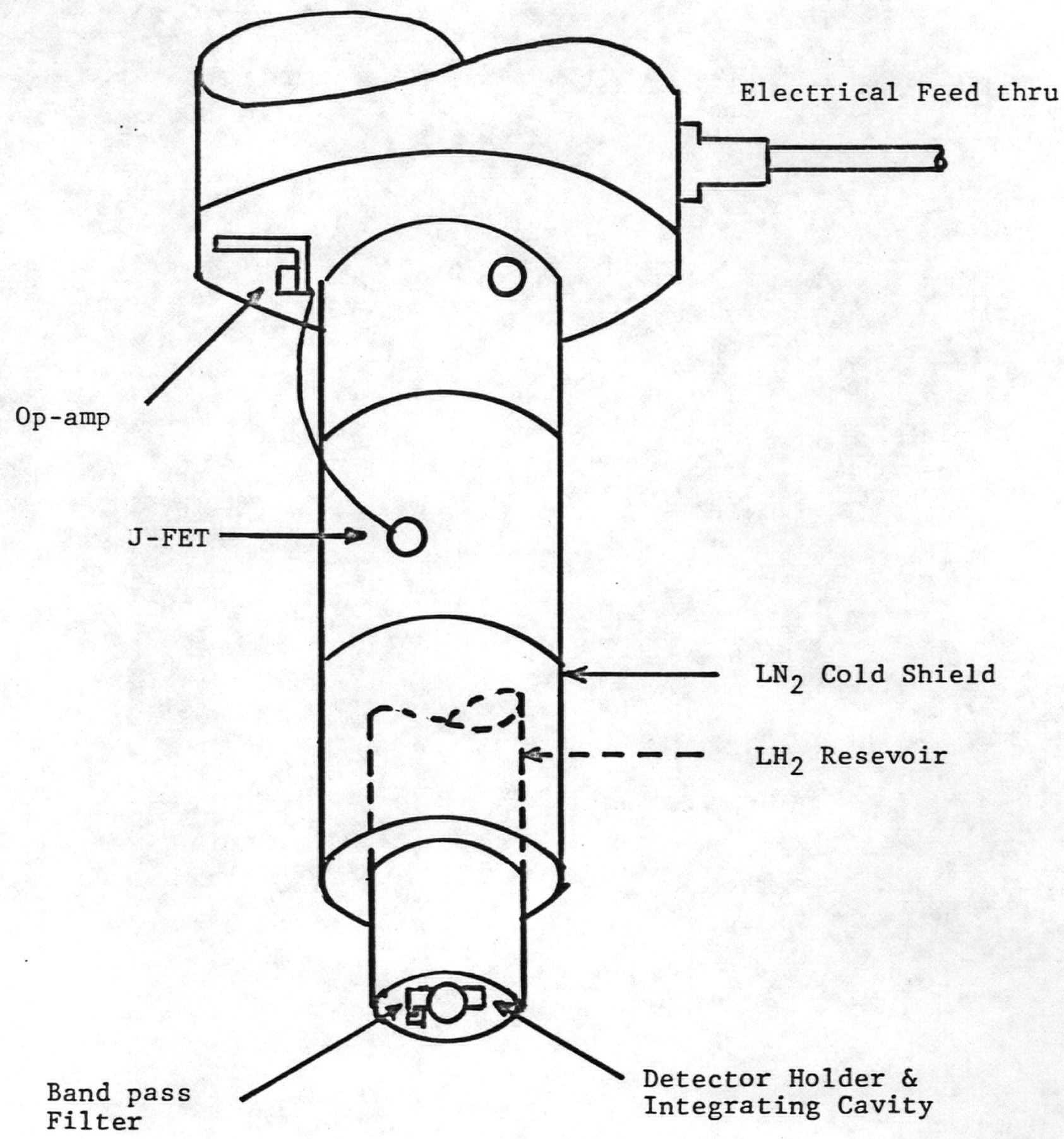

Fig.(3.6) - Cryostat cold shield \& work surface. 
as a mount for the detector's amplifier (with the exception of the operational amplifier which is maintained at room temperature).

A typical two-stage pumping station was used to evacuate the dewar, consisting of a mechanical roughing pump and a diffusion pump with a cold trap. Pressure was monitored by an ionization vacuum gauge of type G1C-110A made by Consolidated Vacuum Corporation. More than twenty hours were required to reach a pressure of $7 \times 10^{-6}$ Torr. This pressure was considered adequate for the experiment, however, because of the inevitable cryopumping due to the liquid helium. This long recycling time proved to be a major obstacle in rendering the detection system operational.

Two electrical feed-throughs were used to accommodate the detector electronics. A standard two-pin feed-through on the bottom of the cover was connected to the power resistor. The eight-pin feed-through on the side of the dewar was constructed by the author to accommodate the remaining electronics.

The inside of the cover of the dewar was painted with black primer paint in order to reduce stray radiation. The inside and outside of the cold shield were also painted black. The black paint reduces the background photon flux and allows for a more accurate calibration of the detector.

\subsection{Detector Electronics}

Figure (3.7) illustrates in block-diagram form the electronic components needed to operate and analyze the experiment. The primary 


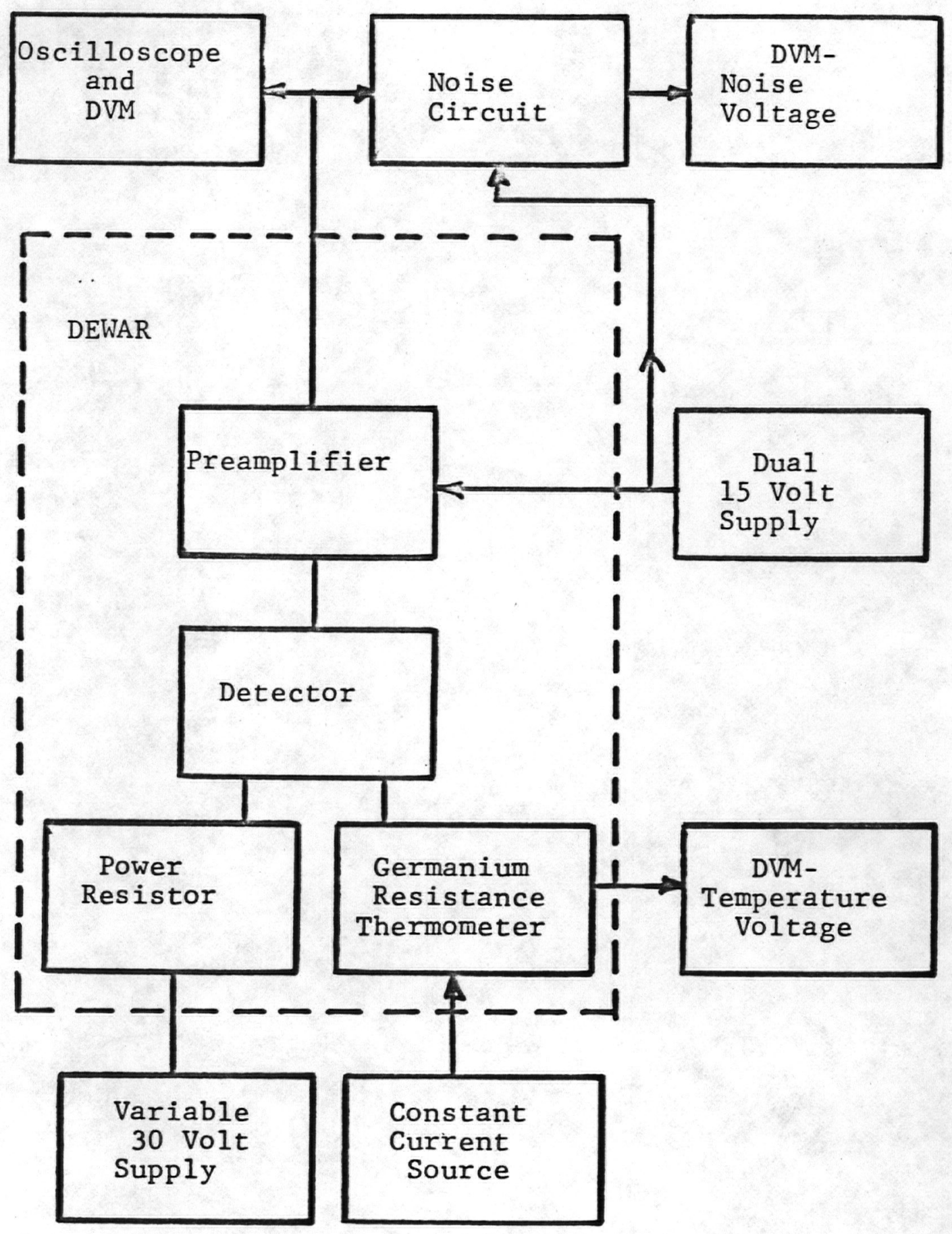

Fig. (3.7) - BLOCK DIAGRAM OF ELECTRONICS 
components include a low noise preamplifier to measure the detector responsivity, a noise circuit to measure the rms noise voltage, and a germanium resistance thermometer. The preamplifier and noise circuits were powered by a dual 15 volt D.C. power supply made in our laboratory. A variable 30 volt power supply regulated the $1 \mathrm{k} \Omega$ power resistor. The constant current source for the thermometer was powered by a 9 volt transistor battery.

The final design of the low-noise preamplifier is illustrated in figure (3.8). It functions as a noninverting amplifier with a voltage gain $\mathrm{G}$, of

$$
G=\frac{V_{\text {OUT }}}{V_{B}}=1+\frac{R_{f}}{R_{D}},
$$

where $V_{\text {OUT }}$ is the output of the preamplifier, $V_{B}$ is the bias voltage of the detector, $R_{f}$ is the feedback resistance, and $R_{D}$ is the detector resistance. Don Langford of Jet Propulsion Laboratory supplied the design from which the final design evolved. Consideration had to be given as to how the detector should be biased. The circuit was designed as to allow one of the detector electrodes to be soldered directly to the pedestal, providing both a ground to complete the bias circuit and excellent thermal contact.

The LM108 operational amplifier manufactured by National Semiconductor was selected for its characteristics of very low noise and a high input impedence of about $10^{14}$ ohms. Such a high input impedence is necessary when coupling the device to detector and feedback resistances on the order of $10^{10}$ ohms. 


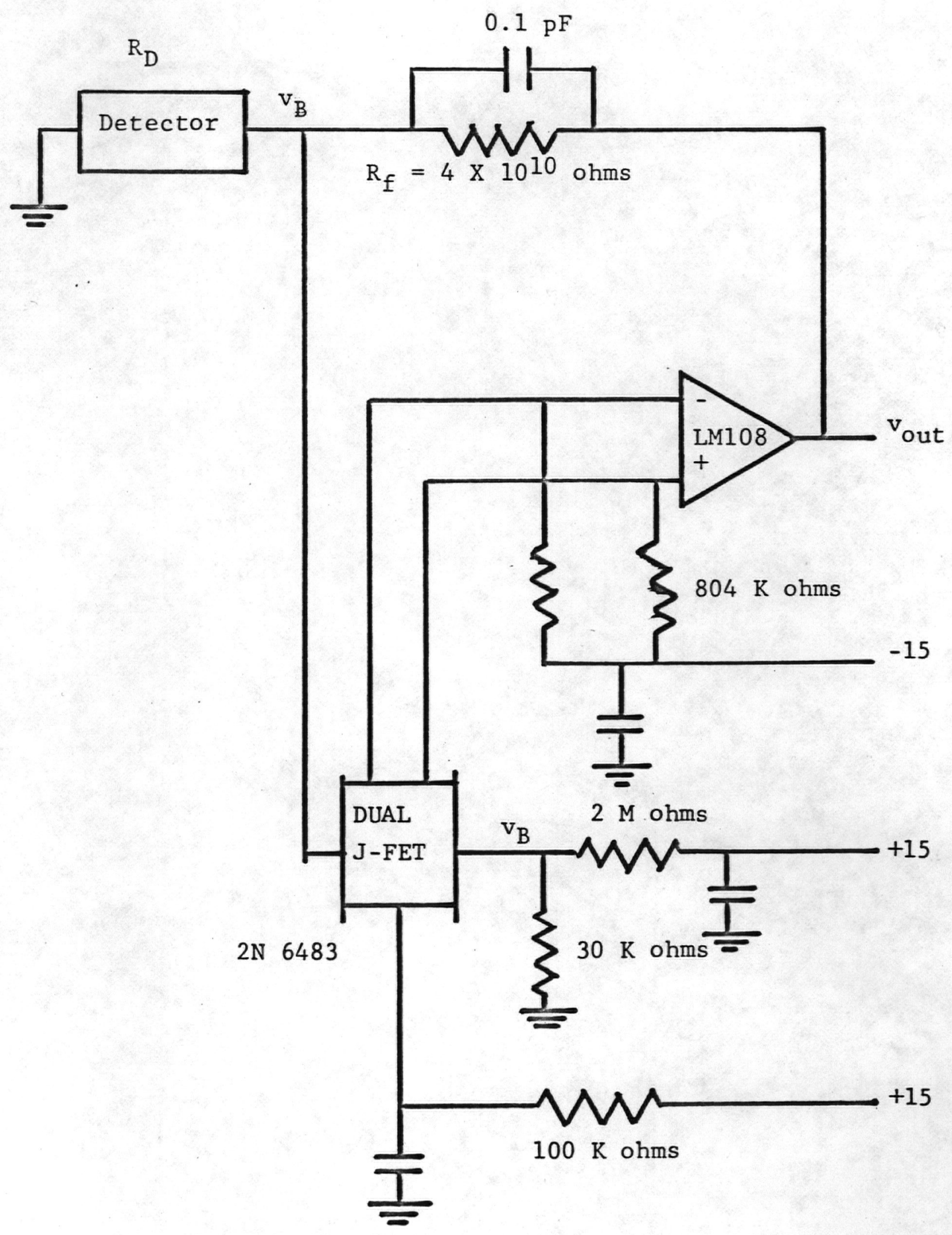

Fig.(3.8) - PREAMPLIFIER CIRCUIT 
The 2 N6483 dual-matched J-FET was also chosen for its low noise characteristics. It functions to minimize unwanted fluctuations between the inputs to the operational amplifier. An $804 \mathrm{k} \Omega$ resistor was placed between each input to the operational amplifier and the -15 volt connection to provide a current path to drive the J-FET.

To prevent the amplifier from being driven into oscillation, a 0.1 pf capacitor was placed in parallel with the $4 \times 10^{9}$ ohm feedback resistor. This resulted in an $\mathrm{RC}$-time constant that limited the spectral content of fluctuations to less than $2.5 \mathrm{kHz}$.

Cooling of the circuit elements further improves noise characteristics of the output signal by reducing the thermal Johnson noise associated with all resistive elements. Because of the extremely high resistance of the feedback resistor, it was maintained at the same low temperature as that of the detector, ranging between $2^{\circ}$ and $5^{\circ}$ Kelvin. The remainder of the circuit, with the exception of the operational amplifier, was attached with teflon tape to a copper tube that fit over the outside of the cold shield. The components were maintained at a temperature approaching $77^{\circ}$ Kelvin. The operational amplifier was maintained at room temperature because it would not function at $77^{\circ}$ Kelvin. Consideration had to be given to the temperature coefficient of the biasing resistors, as cooling them resulted in a 0.07 volt increase in the bias voltage to $V_{B}=0.2792$ volts.

The responsivity and noise properties of the detector are determined from equation (3.1). Rearrangement of equation (3.1) results in a detector resistance of 


$$
R_{D}=\frac{R_{f}}{v_{\text {OUT }} / V_{B}-1} .
$$

The current $I_{D}$ passing through the detector is given by Ohm's Law to be

$$
I_{D}=\frac{V_{B}}{R_{D}}
$$

The current noise $I_{N}$ from the detector is given by

$$
I_{N}=\frac{V_{N}}{R_{D}}\left(\frac{V_{B}}{V_{\text {OUT }}}\right)
$$

where $V_{N}$ represents the detectors contribution to the total noise voltage from all sources as measured by the noise circuit.

Figure (3.9) is a schematic of the noise circuit that was designed to have an output proportional to the root-mean-square of the voltage fluctuations in the output of the preamplifier. The primary component is an integrated circuit from Analog Devices (type AD536AJD) that acts as a true rms-to-dc voltage converter. To assure that the device was operating in its linear region, an amplifier with a gain of 100 was used on the input stage of the converter. The noise circuit was powered by the same dual 15 volt power supply as the preamplifier.

A germanium resistance thermometer from LakeShore Cryotronics Inc. (type GR-200B-1000) was selected as a temperature measuring device. It was used in a four lead potentiometric configuration 


$$
\text { Gain }=\left(R_{1} / R_{2}+1\right)=100
$$

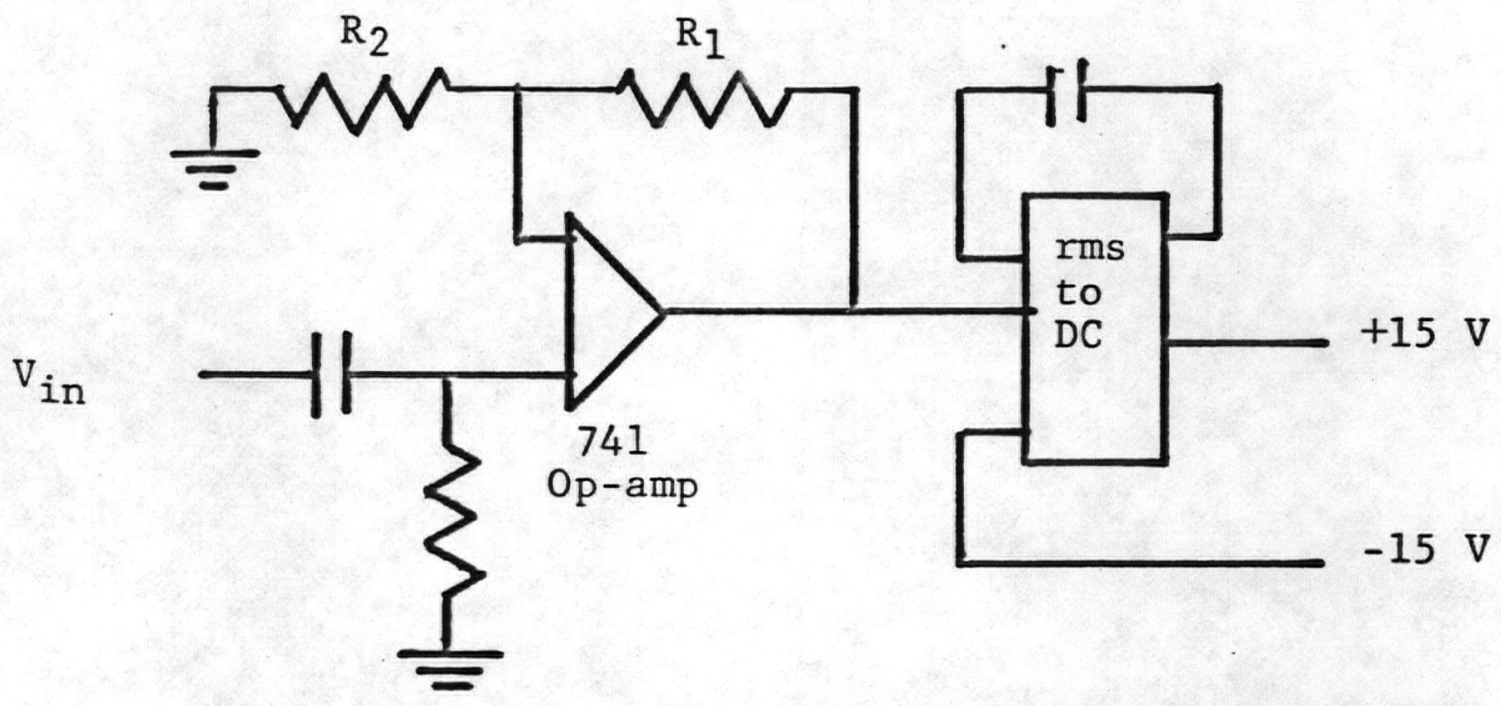

Fig.(3.9) - TRUE RMS TO DC NOISE CIRCUIT WITH A GAIN OF 100 
where two of the leads pass a constant current through the device and the remaining two leads measure the voltage drop across the resistive element. This configuration reduces the resistive heating that would normally be present in a two wire device. The constant currents ranged from $680 \mu \mathrm{A}$ down to $0.76 \mu \mathrm{A}$ to accommodate temperatures from $300^{\circ}$ Kelvin down to $2^{\circ}$ Kelvin.

Two different calibration methods were employed in two different temperature regimes. For temperatures ranging from $4.2^{\circ}$ Kelvin to $40^{\circ}$ Kelvin our thermometer was calibrated against a similar device borrowed from Professors W. Johnson and D. Douglas of the Department of Physics and Astronomy at the University of Rochester. For temperatures below $4.2^{\circ}$ Kelvin, the resistance $R$, of germanium is assumed to increase as

$$
R=R_{0} \exp (A / T),
$$

where $R_{0}$ and $A$ are constants and $T$ is the temperature in degrees Kelvin. This equation is represented by a straight line when plotted on semi-log paper. The two unknown parameters $R_{0}$ and $A$ can be determined by measuring the resistance at two different temperatures. One temperature was obviously $4.2^{\circ}$ Kelvin for which we accurately knew the resistance. The second temperature chosen was the $\lambda$-point of liquid helium which occurs at $2.17^{\circ}$ Kelvin. At its $\lambda$-point, liquid helium ceases to boil and becomes a super fluid. To determine at what point this occurred, a glass plate was placed at the top of the helium reservoir which was being pumped on. The reduced pressure caused the boiling temperature of the helium to drop and the point at 
which it stopped boiling was clearly visible through the plate glass. The values $R_{0}=177$ and $A=6.3$ were determined to correspond to the LakeShore device.

\subsection{Filter and Window Materials}

Polyethylene was selected as the window material for the dewar. It is characterized as having no appreciable absorption bands greater than 14 microns and has a transmission of approximately $80 \%$ between 60 and 100 microns for a thickness of $0.3 \mathrm{~mm} .{ }^{4}$ The polyethylene window used was approximately $1.6 \mathrm{~mm}$ in thickness which resulted in a transmission of about $30 \%$. The window had to be thick in order to resist the force exerted on it by atmospheric pressure when the dewar was under vacuum.

A BPF-2 far infrared band-pass filter made by Descriptive Design and Development Corporation was selected for use at 100 microns. The filter and the polyethylene window material were borrowed from Professor Judith Pipher of the department of Physics and Astronomy at the University of Rochester. Figure (3.10) shows the transmission profile of the filter. Its band-pass is between 44 and 100 microns with a peak transmittance of $42 \%$ at 62 microns. $^{5}$ The transmittance of this filter is good between 60 and 90 microns, ranging from $42 \%$ to $25 \%$ respectively. The resulting transmission of the window and filter ranges from a maximum of $12.6 \%$ at 60 microns, $7.5 \%$ at 90 microns, down to zero at 100 microns. 


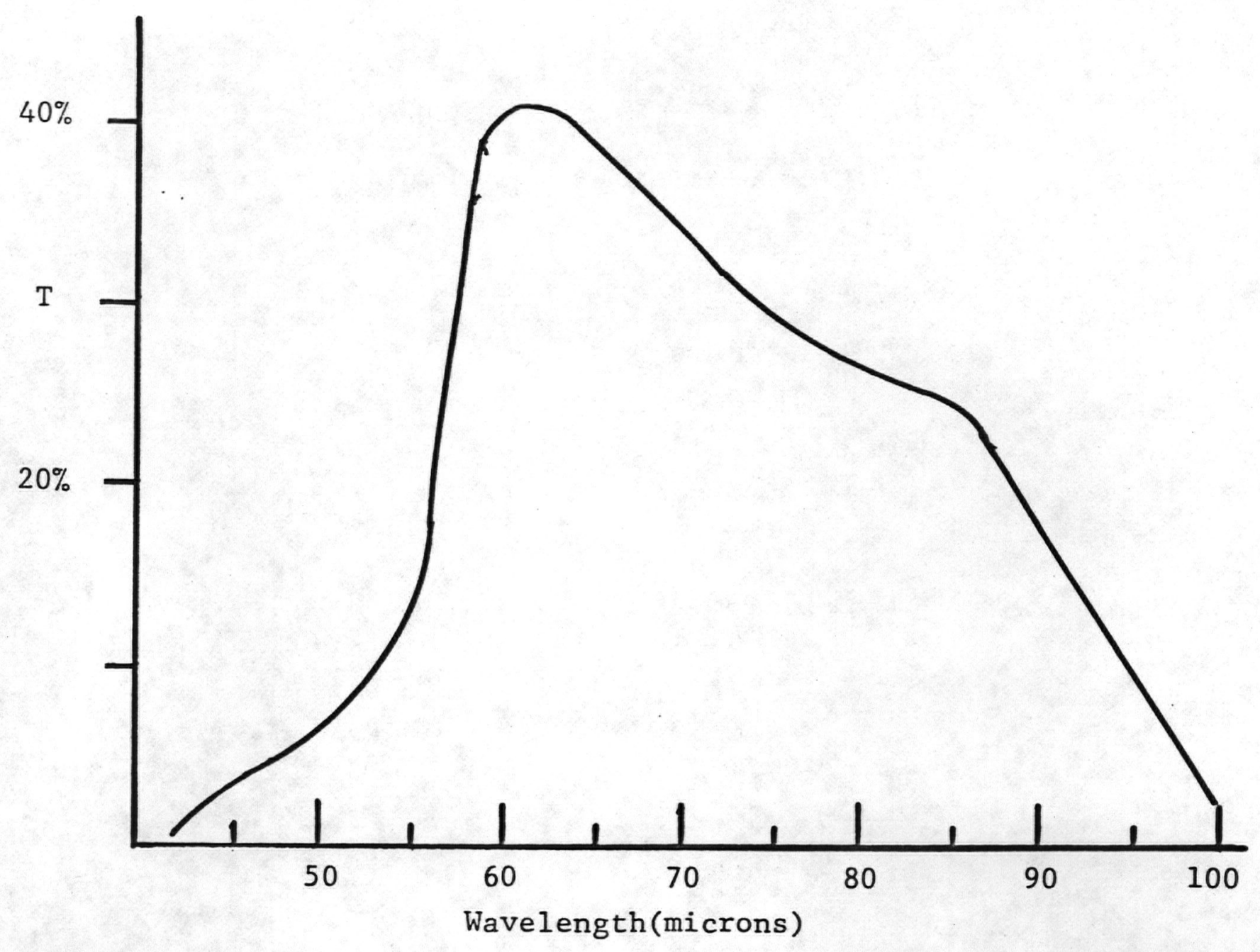

Fig.(3.10) - FILTER BAND-PASS 
It is obvious that this is not the ideal filter for 100 micron detection, but it is adequate in the range from 60 to 90 microns. Unfortunately, filters with a peak transmittance at 100 microns were not available to the author at the time of this research. 


\section{REFERENCES}

1. P.R. Bratt, "Ge:Ga and Ge:Be Photoconductive Detectors for Far Infrared Astronomy from a Space Platform," (SPIE Vol. 132, 1978).

2. L.S. Varnell and D.E. Langford, "Radiation Effects in IRAS Extrinsic Infrared Detectors," (Jet Propulsion Laboratory, Pasadena, Ca., June 1982).

3. W.J. Moore, "100 Micron Detector Development Program," (Ames Research Center, NASA, March 1976).

4. Y. Yamamada, "Transmission Filters in the Far-Infrared Region," (Journal of the Optical Society of America, Vol. 52 no. i, January 1962).

5. S.P. Varma, "Far Infrared Band-Pass Filters in the $400-16 \mathrm{~cm}^{-1}$ Spectral Region," (Applied Optics, Vol. 8, October 1969) page 2151.

6. P. Horowitz and W. Hill, The Art of Electronics, (Cambridge University Press, 1980). 
CHAPTER 4

EXPERIMENTAL RESULTS

\subsection{Introduction}

Test procedures which led to system optimization are discussed in the first section of this chapter. The second section presents experimental data for the dark current as a function of the detector temperature. A comparison is made to the theoretical predictions presented in chapter 2. This is followed by a comparison of theory and experiment for the mean-square current noise as a function of temperature. Finally, data is presented for the current responsivity, both as a function of the blackbody temperature and the total power incident on the detector.

\subsection{Initial Testing and System Optimization}

System optimization focused primarily on the preamplifier and sources of undesirable noise. As was discussed in chapter 3 , the 
inefficient vacuum system slowed the optimization process considerably.

During the first run of the experiment, the preamplifier output remained saturated at approximately $14.45 \mathrm{~V}$. Two plausible explanations came to mind; (1) the feedback resistance was too high and (2) the preamplifier would not function properly at $77^{\circ}$ Kelvin.

A Keithley Instruments 601 Electrometer was employed to measure resistances of the order of $10^{8}$ to $10^{10}$ ohms. A resistance of $4.4 \times 10^{8}$ ohms, which was thought to be lower than the desired feedback resistance, was used on the subsequent trial to assure that the preamplifier gain would not be saturated.

Evaluation of the test circuit revealed that the LM108 operational amplifier would not function at $77^{\circ}$ Kelvin. Thus, the operational amplifier was mounted to a room-temperature copper bracket that was soldered to the inside of the helium cryostat.

The next run of the system proved to be successful and data indicated that the detector resistance was on the order of $10^{8}$ ohms at $4.2^{\circ}$ Kelvin. Three of the feedback resistors acquired from Jet Propulsion Laboratory were connected in parallel, resulting in a feedback resistance of $R_{f}=4 \times 10^{9}$ ohms. This resistance resulted in voltage saturation of the amplifier at a temperature of $6^{\circ}$ Kelvin.

Considerable effort was devoted to locating and minimizing sources of undesriable noise. Unwanted noise resulted from mechanical vibrations, electrical pickup, and ground loops. The last two problems were cured by shielding all signal wires and bringing all ground leads to a common point. The reason for the appreciable sen- 
sitivity to mechanical vibrations was never completely understood. It may arise from compression of the crystal lattice resulting in a voltage change associated with the piezo-electric effect. Four inertubes were placed beneath a 250 pound steel plate on which the cryostat was magnetically mounted. This served to isolate the detector from the mechanical vibrations of its environment. The isolation table was effective, but did not entirely eliminate the problem of mechanical pick-up.

\subsection{Experimental Results}

The dark current as a function of the detector temperature was determined from the preamplifier output data by use of equations (3.2) and (3.3). The detector temperature ranged between $2^{\circ}$ and $5^{\circ}$ Kelvin. A high volume vacuum pump was connected to the helium reservoir to permit temperatures below $4.2^{\circ}$ Kelvin to be reached. A $1 \mathrm{k} \Omega$ power resistor attached to the detector holder and connected to a $30 \mathrm{~V}$ power supply was employed to reach temperatures in excess of 4. $2^{\circ}$ Kelvin. Heating due to the power resistor resulted in a temperature gradient between the LakeShore thermometer and the detector element. The experimental data above $4.2^{\circ}$ Kelvin was thus shifted up in temperature resulting in a discontinuous curve when plotted. Decreasing the experimental values of the temperature by $1 / 3^{\circ}$ Kelvin for data taken with the power resistor on resulted in a continuous function. These corrected data are assumed to be the true experimental values and are used in the following analysis. 
The theoretical value of the mean current passing through the detector is given by equation (2.21) to be

$$
\bar{i}=\frac{e V}{L^{2}}\left[\mu_{n} \bar{N}+\mu_{p} \bar{P}\right] .
$$

Since gallium is an acceptor impurity in Ge:Ga, $\mu_{n} \bar{N}$ will be much less than $\mu_{p} \bar{P}$. The resulting theoretical detector current is given by

$$
\bar{i}=\frac{e V}{L^{2}} \mu_{p} \bar{p}
$$

which is expressed in terms of the fractional ionization as

$$
\bar{i}=\frac{e V}{L^{2}} \mu_{p}\left(N_{a}-N_{d}\right) f .
$$

The following material parameters for the Ge:Ga detectors used in this experiment are quoted from a report by Jet Propulsion Laboratory: ${ }^{1}$

$$
\begin{aligned}
& \mu_{p}=50 \mathrm{~m}^{2} \mathrm{v}^{-1} \mathrm{sec}^{-1} \\
& \mathrm{~L}=1.4 \times 10^{-3} \mathrm{~m} \\
& \mathrm{~N}_{\mathrm{d}}=1.58 \times 10^{9} \text { donors } \\
& \mathrm{N}_{\mathrm{a}}=7.88 \times 10^{11} \text { acceptors. }
\end{aligned}
$$

The detector bias as stated in chapter 3 was $V_{B}=0.2792 \mathrm{~V}$. These parameters result in a theoretical current given by

$$
\bar{i}=(0.9) f[A],
$$


where again $f$ is the fractional ionization of available acceptor impurities. Figure (4.1) illustrates the fractional ionization versus temperature in Ge:Ga between the temperatures of $0^{\circ}$ and $50^{\circ}$ Kelvin. Note that $f$ begins to saturate at about $10^{\circ}$ Kelvin.

Experimental data for the detector dark current in nanoamps versus the detector temperature in degrees Kelvin is indicated by the octogonal marks in figure (4.2). The solid lines represent the best theoretical predictions of the dark current as explained below. Equation (2.34) gives the functional dependence of the fractional ionization on the thermal generation rate as

$$
f=\left(\frac{1}{1-\lambda}\right)\left\{\frac{-1}{2}\left(\Gamma_{t h}+\lambda\right)+\left[\frac{1}{4}\left(\Gamma_{t h}-\lambda\right)^{2}+\Gamma_{t h}(1-\lambda)\right]^{\frac{3}{2}}\right\}
$$

where the thermal generation rate is given by equation $(2.41)$ to be

$$
\Gamma_{\text {th }}=\Gamma_{0}\left(k T / \varepsilon_{a}\right)^{3 / 2} \exp \left(-\varepsilon_{a} / k T\right) .
$$

The material dependent parameter $\Gamma_{0}$ is given by equation $(2.40)$ to be

$$
r_{0}=\frac{V}{N_{a}}\left(\frac{2 \pi m \varepsilon}{h^{2}}\right)^{3 / 2}
$$

It should be noted that our experimental data for the dark current as a function of temperature does not match perfectly with the theoretical predictions. There are, however, distinct correlations between experiment and theory as is evidenced by figure (4.2). Careful consideration must be given as to how these differences may have come about. Note that equation (4.7) contains two free para- 


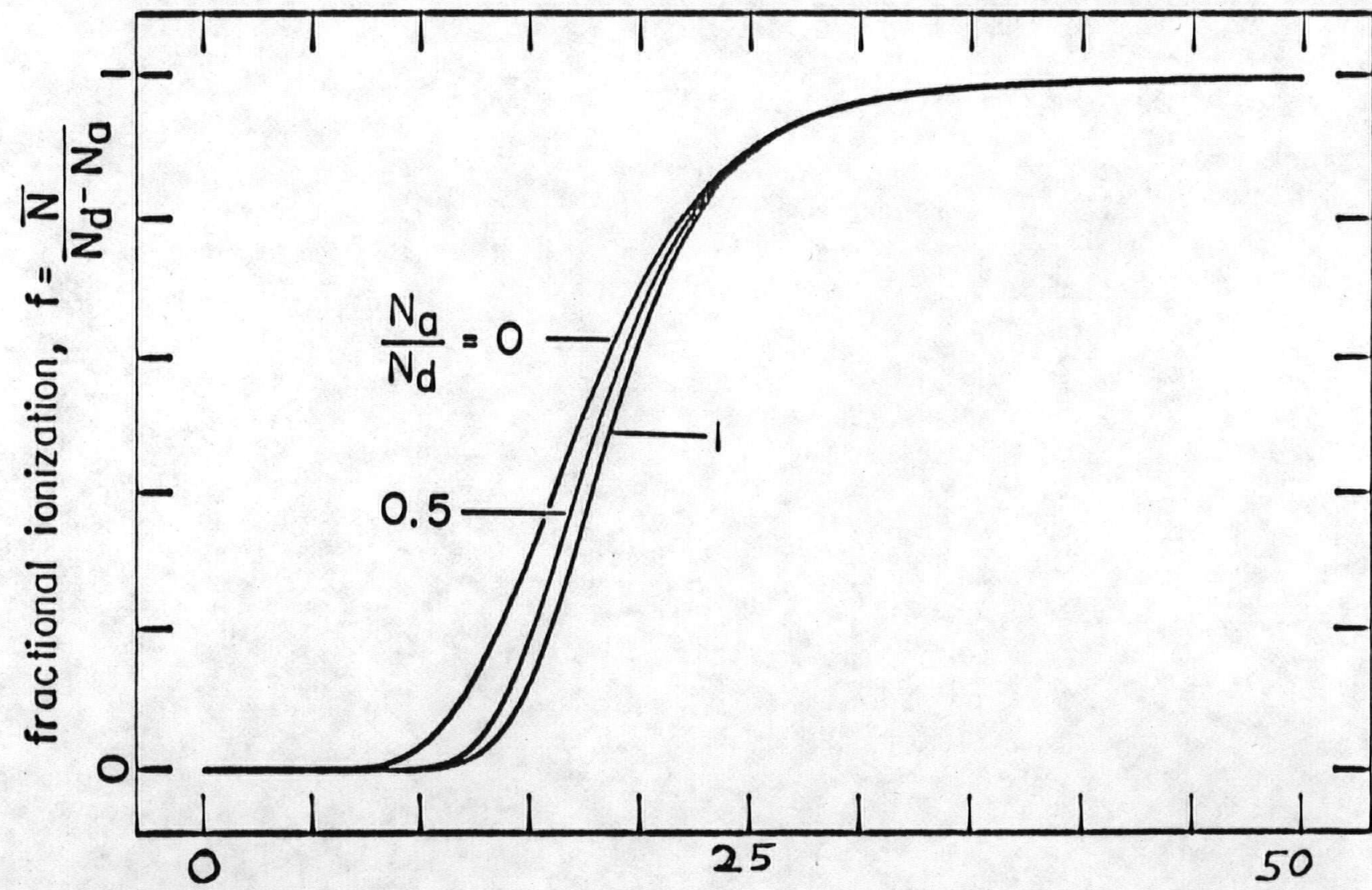

TEMPERATURE (DEGREES KELVIN)

Fig.(4.1) - Fractional ionization versus temperature. 


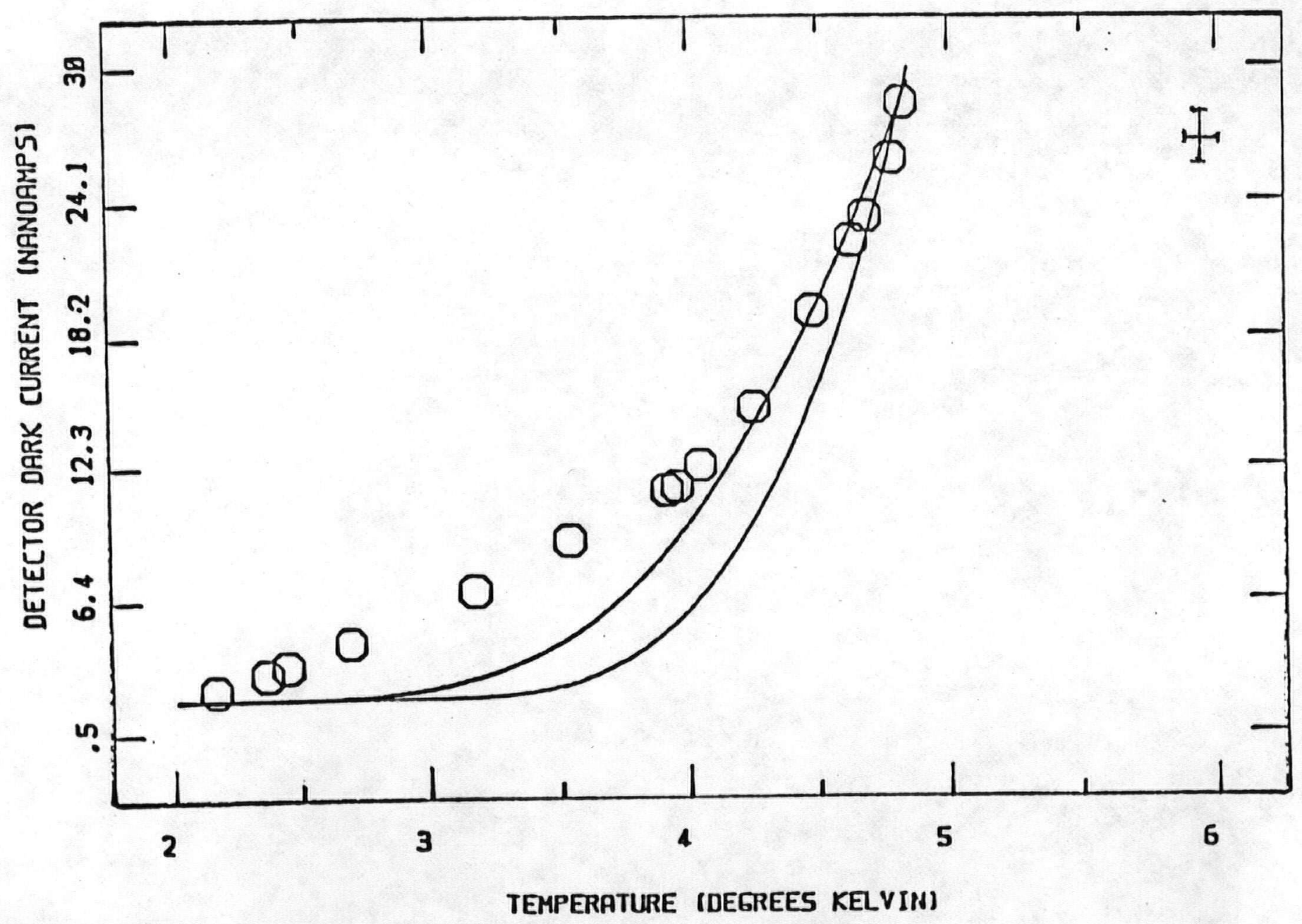

Fig.(4.2) - Dark current versus temperature. 
meters; the acceptor density $\mathrm{N}_{\mathrm{a}} / \mathrm{V}$ and the ionization energy $\varepsilon_{\mathrm{a}}$. Equation (4.7) is exponentially dependent on the ionization energy $\varepsilon_{\mathrm{a}}$. Thus, even a small change in this value will produce a noted effect on the fractional ionization. Perhaps it is more accurate to consider the acceptor level to span over a finite energy band rather than a discrete energy level. With this in mind, the lower curve in figure (4.2) represents the ionization energy value of $\varepsilon_{a}=0.0108 \mathrm{eV}$ and the upper solid line represents a $35 \%$ decrease in this value. The resulting curve is seen to conform more closely to the experimental data. Changing the number of acceptors had a much smaller effect and is not shown.

A constant current was also added to the theoretical curve which could be manifested by phenomenon such as leakage current, background photon flux, impact ionization, or thermal conduction through the detector leads. Due to the high resistance of the detector, even a fingerprint could result in a considerable leakage current. The detector leads could also be responsible for a leakage current. A contribution to the current could also result from the background photon flux from the cold shield which is at $77^{\circ}$ Kelvin, a temperature considerably higher than that of the detector. The bias voltage and the inter-electrode distance lead to an electric field strength of approximately $2 \mathrm{~V} / \mathrm{cm}$. According to a report from Santa Barbara Research Center, ${ }^{2}$ impact ionization occurs at an electric field strength on the order of $3 \mathrm{~V} / \mathrm{cm}$ for these detectors. This phenomenon may have resulted in the ionization of an appreciable number 
of acceptors, which would lead to a decrease in the resistance of the detector and thus an increase in the current.

It is important to point out that temperature readings between $4.2^{\circ}$ and $2.17^{\circ}$ Kelvin may be slightly in error, as our assumption of the functional form of the resistance in this domain may not be entirely correct.

We have introduced a number of probable causes for the discrepancies between our experiment and theory, but it is difficult to tell which of these factors plays a dominant role, as their effects are often indistinguishable from each other.

As data was available for similar Ge:Ga detectors from Santa Barbara Research Center, ${ }^{3}$ it seemed reasonable to compare the data to our theory. This comparison is illustrated in figure (4.3). Their detectors were under a background flux of $1.2 \times 10^{9}$ photons $\mathrm{sec}^{-1} \mathrm{~cm}^{-2}$ which is manifest by a constant current contribution. The lower solid curve results from a best estimation of the material parameters and the upper solid curve results from a $50 \%$ decrease in the value of the ionization energy. These curves appear to be in good agreement with our theory.

Figure (4.4) illustrates a comparison between experimental data and theory for the mean-square dark current noise as a function of detector temperature. It should be pointed out that this data did not come from the same detector as the one from which the responsivity data came. Again, the lower curve represents the theory based on the best estimation of material parameters. The upper solid curve 


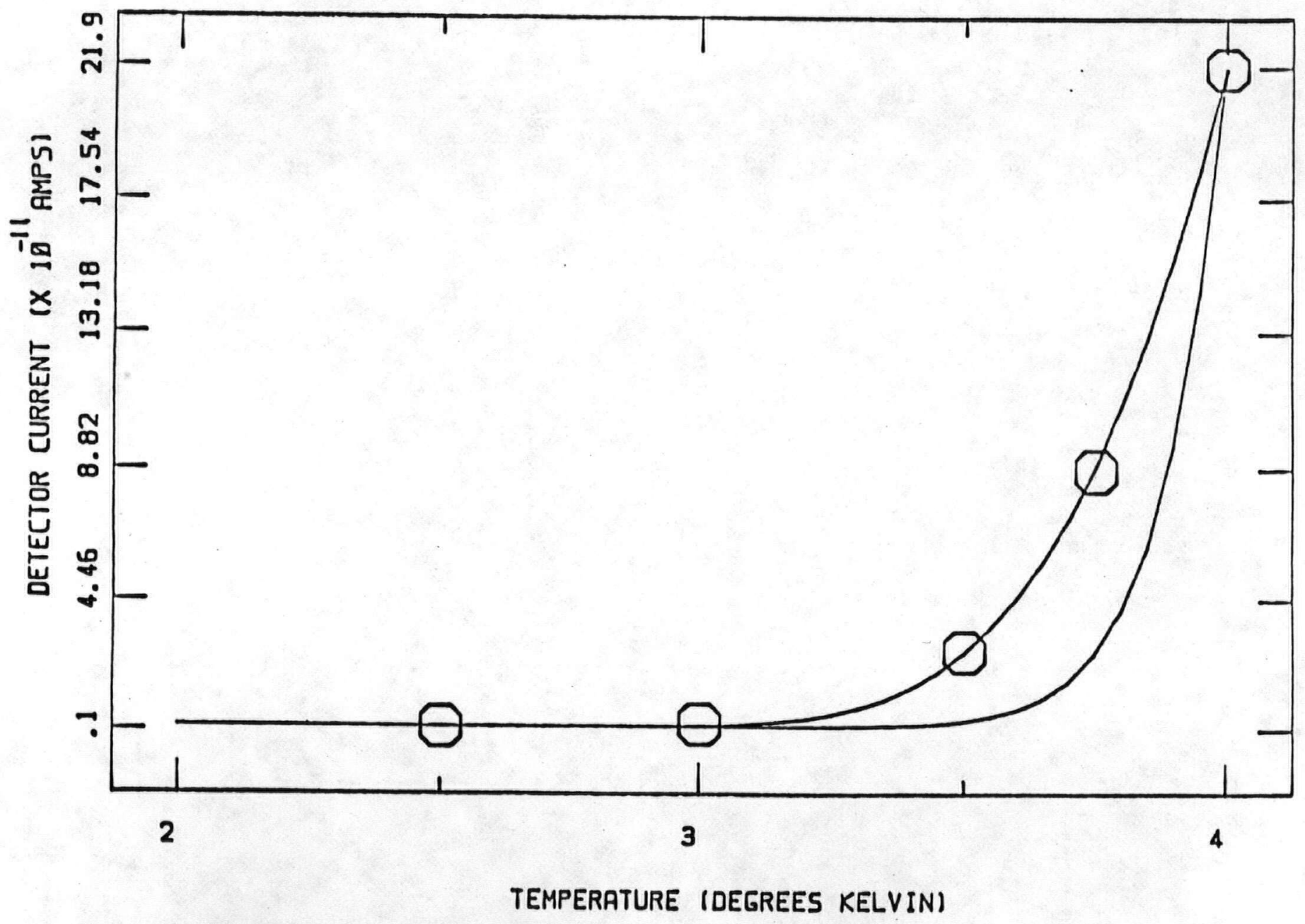

Fig. (4.3) - SBRC data for dark current versus temperature. 


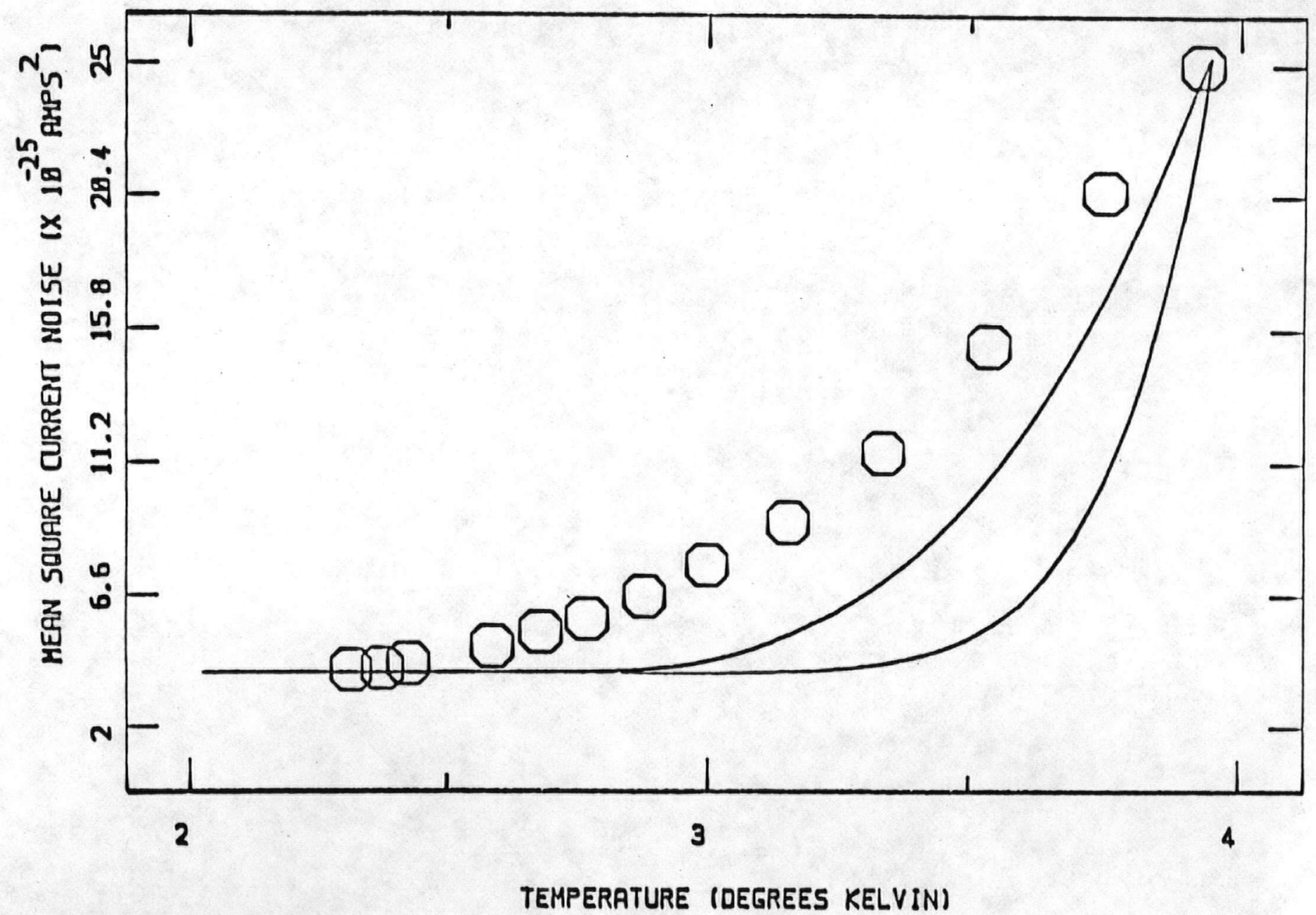

Fig.(4.4) - Mean-square dark current noise versus temperature. 
results from decreasing the value of the ionization energy by $35 \%$ from that of the lower curve.

A constant term is added to the theoretical curves in figure (4.4) to account for such noise sources as amplifier noise, electrical pick-up, and mechanical vibrations. The only appreciable noise from the amplifier was due to the Johnson noise of the bias resistor which amounted to $1.4 \times 10^{-25} \mathrm{~A}^{2}$. This would not effect the shape of the curve, but would increase the current by a constant additive factor. Electrical pick-up was minimized by proper shielding of the electronics and the electrical cables. As mentioned above, noise due to mechanical vibrations was reduced by the isolation table, but was still evident. It is assumed that these fluctuations average over time to result in a constant current noise contribution.

The Johnson noise due to the detector and load resistances does lead to a non-constant contribution to the noise current. This could play an important role in the differences between theory and experiment. This noise current is given by ${ }^{4}$

$$
\bar{i}_{N_{\text {Johnson }}^{2}}=\frac{4 k T}{R_{d}}+\frac{4 k T}{R_{f}} .
$$

The Johnson noise due to the feedback resistor increases only with an increase in detector temperature. The Johnson noise associated with the detector resistance, however, increases with an increase in detector temperature and with the associated decrease in detector resistance. This noise contribution reaches a maximum of only 
$3 \times 10^{-29} A^{2}$ due to the low detector temperature and to the high detector and feedback resistances. Thus it does not constitute a large contribution to the total noise.

The final stage of experimentation involved photon flux responsivity measurement as a function of blackbody temperature and the total power incident on the detector. A blackbody source at a distance of 7 inches and having an aperature of 1 inch in diameter was varied between the temperatures of $473^{\circ}$ and $1073^{\circ}$ Kelvin. The blackbody had to be positioned as close to the dewar window as possible because of the high absorption of 100 micron radiation by water molecules. This requirement presented difficulties in collecting good data due to the inevitable heating of the cryostat and detector. Due to the large photon flux on the detector, detector holder, and work surface, temperatures below $3.3^{\circ}$ Kelvin could not be reached. Two data points were taken at each blackbody temperature. One was taken with a mirror in front of the window to monitor the dark current and one measurement was taken without the mirror to determine the flux responsivity. Note that the dark current increased with increasing blackbody temperature due to heating of the detector.

To determine the responsivity it is first necessary to calculate the power $P$ incident on the detector. The power is given by

$$
P=L A F \Omega \text {, }
$$

where $L$ is the radiance in a wavelength interval between 60 and 90 microns, $A$ is the effective area of the detector, $F$ is the average 
transmittance of the air, window, and filter $(F=0.05)$, and $\Omega$ is the limiting solid angle. The integrating cavity defines a solid angle of approximately $2 \times 10^{-2} \mathrm{sr}$. The blackbody aperature subtends a solid angle of only $1.5 \times 10^{-2} \mathrm{sr}$. The power falling on the detector must include both a contribution due to the blackbody and a contribution due to the $300^{\circ}$ Kelvin background.

The total radiance is given by $L=M / \pi$ where $M$ is the radiant exitance given by

$$
M=\sigma T^{4},
$$

where $\sigma$ is known as the Stephan-Boltzman constant and is equal to $5.67 \times 10^{-8} \mathrm{~W} / \mathrm{m}^{2} \mathrm{~K}^{4}$, and $T$ is the temperature in degrees Kelvin. The fraction of the total radiance contained between the wavelengths of 60 and 90 microns was calculated from a table of the Planck function ${ }^{5}$ for a given blackbody temperature. The power due to the $300^{\circ} \mathrm{Kelvin}$ background was found to be approximately $4.5 \times 10^{-8} \mathrm{~W}$.

We assume that the current responsivity $R$ is of the form

$$
R=\frac{I_{p h}-I_{t h}}{P_{T}}
$$

where $I_{p h}$ is the current in the presence of the photon field, $I_{t h}$ is the dark current, and $P_{T}$ is the total power incident on the detector. Figures (4.5) and (4.6) represent the increase in the current through the detector due to photon flux as functions of the blackbody temperature and the total incident power, respectively. Notice that the total incident power and the blackbody temperature scale by approxi- 


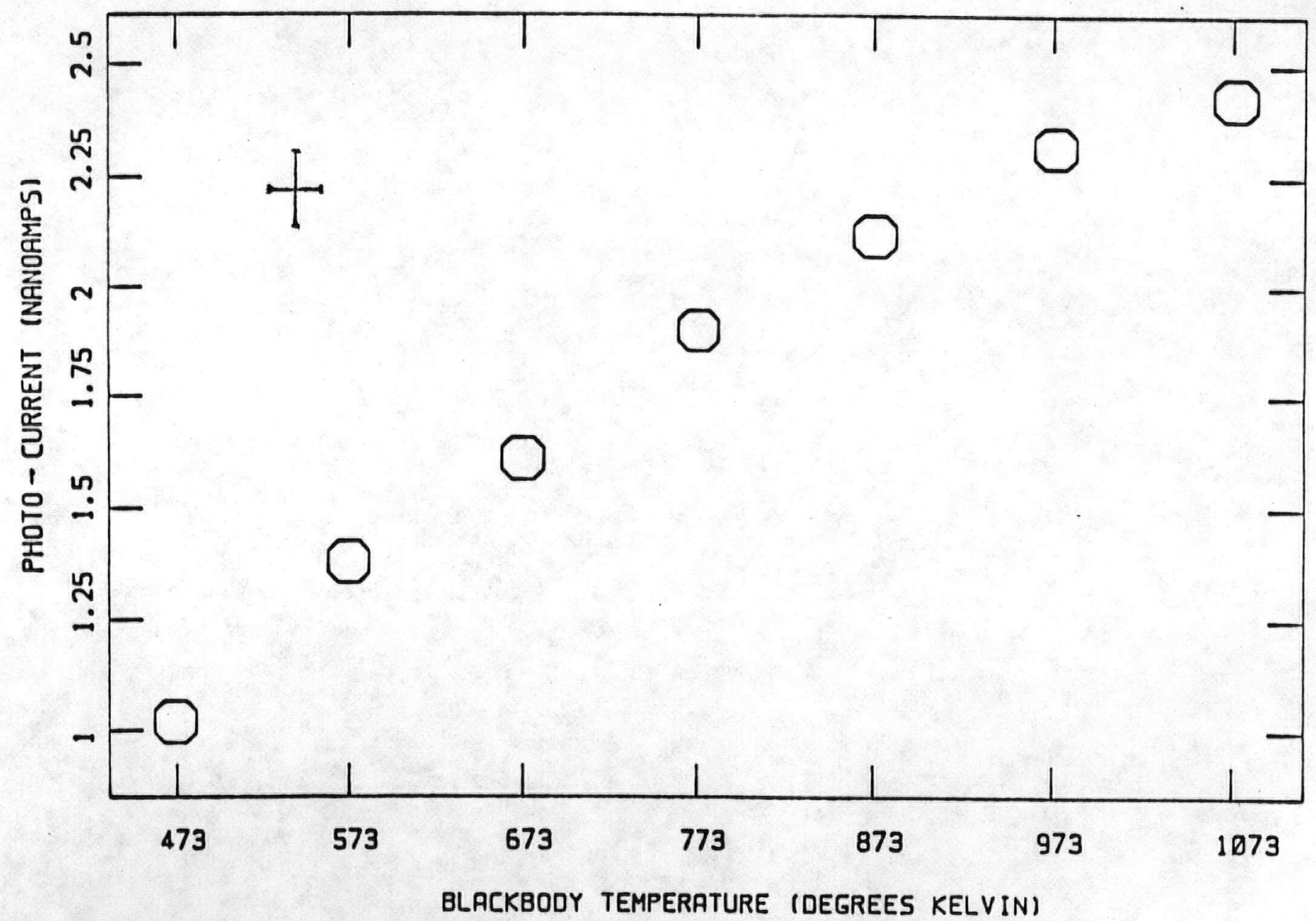

Fig.(4.5) - Photocurrent versus blackbody temperature. 


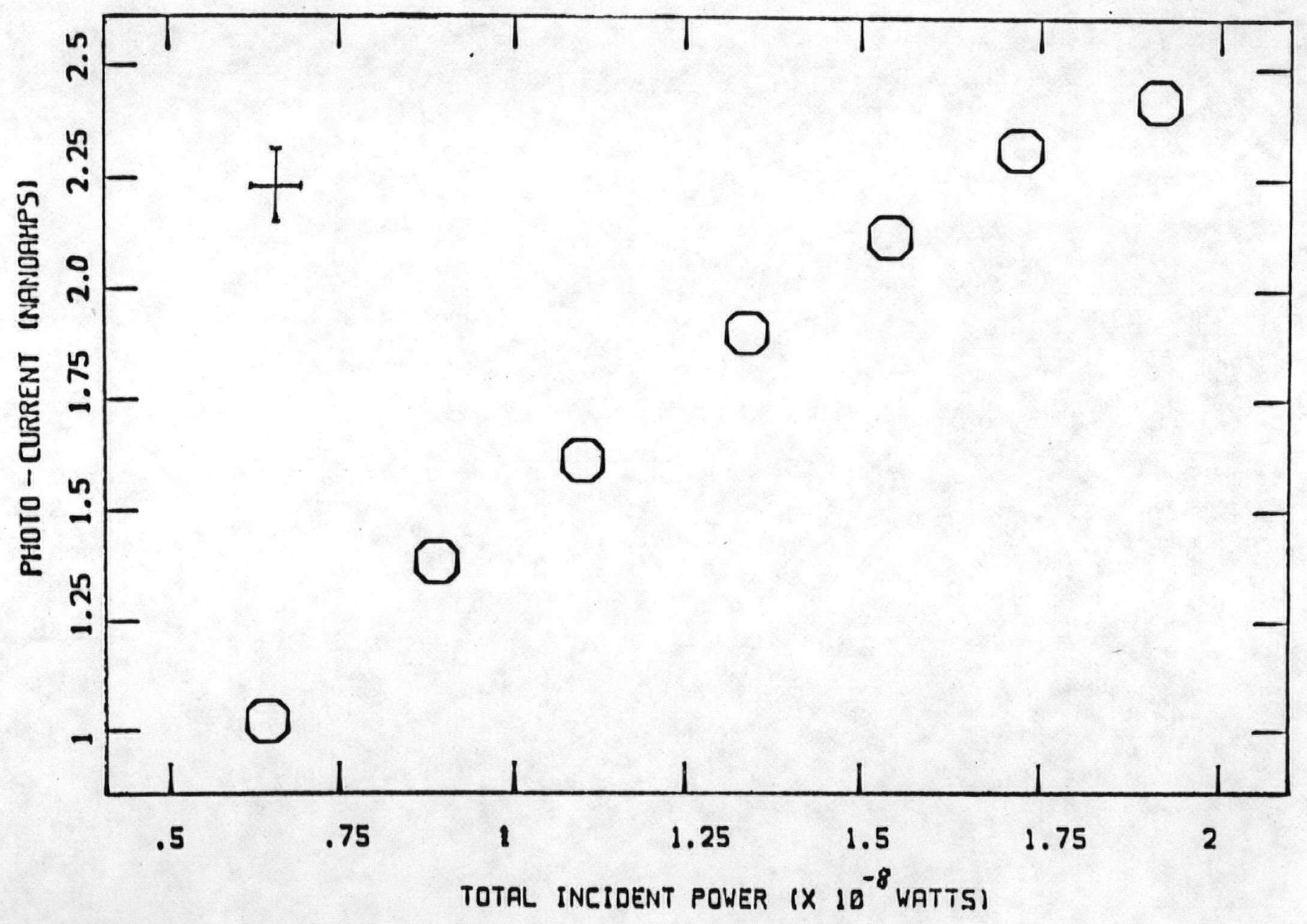

Fig.(4.6) - Photocurrent versus total incident power. 
mately equal amounts with respect to the photo-induced current. This is a consequence of the Rayleigh-Jeans radiation law in the limit hv $\ll$ kT. Current responsivity is plotted as functions of the blackbody temperature and the total incident power in figures (4.7) and (4.8), respectively.

These four plots appear to be in agreement with theory. If we look at figure (2.4) of the fractional ionization as a function of the dimensionless generation rate, we see a functional dependence similar to that of figures (4.5) and (4.6). Again, the fractional ionization is proportional to the current. The dependence of the dimensionless generation rate on the photon flux is not known, as we do not know the functional form of the recombination coefficient $p$. The responsivity in figures (4.7) and (4.8) is seen to decrease with increasing flux, a dependence which is supported by figure (2.21) of the responsivity as a function of the dimensionless generation rate $\Gamma$.

The noise equivalent power, or NEP, was measured under conditions in which the detector was viewing a $473^{\circ}$ Kelvin blackbody source. The NEP is given by

$$
N E P=\frac{I_{N}}{R} .
$$

According to the theory presented in chapter 2, the current noise does not distinguish between photon and thermal generation rates of equal magnitude. It only depends on the magnitude of the dimensionless generation rate $\Gamma$. Thus, the noise current from previous dark 


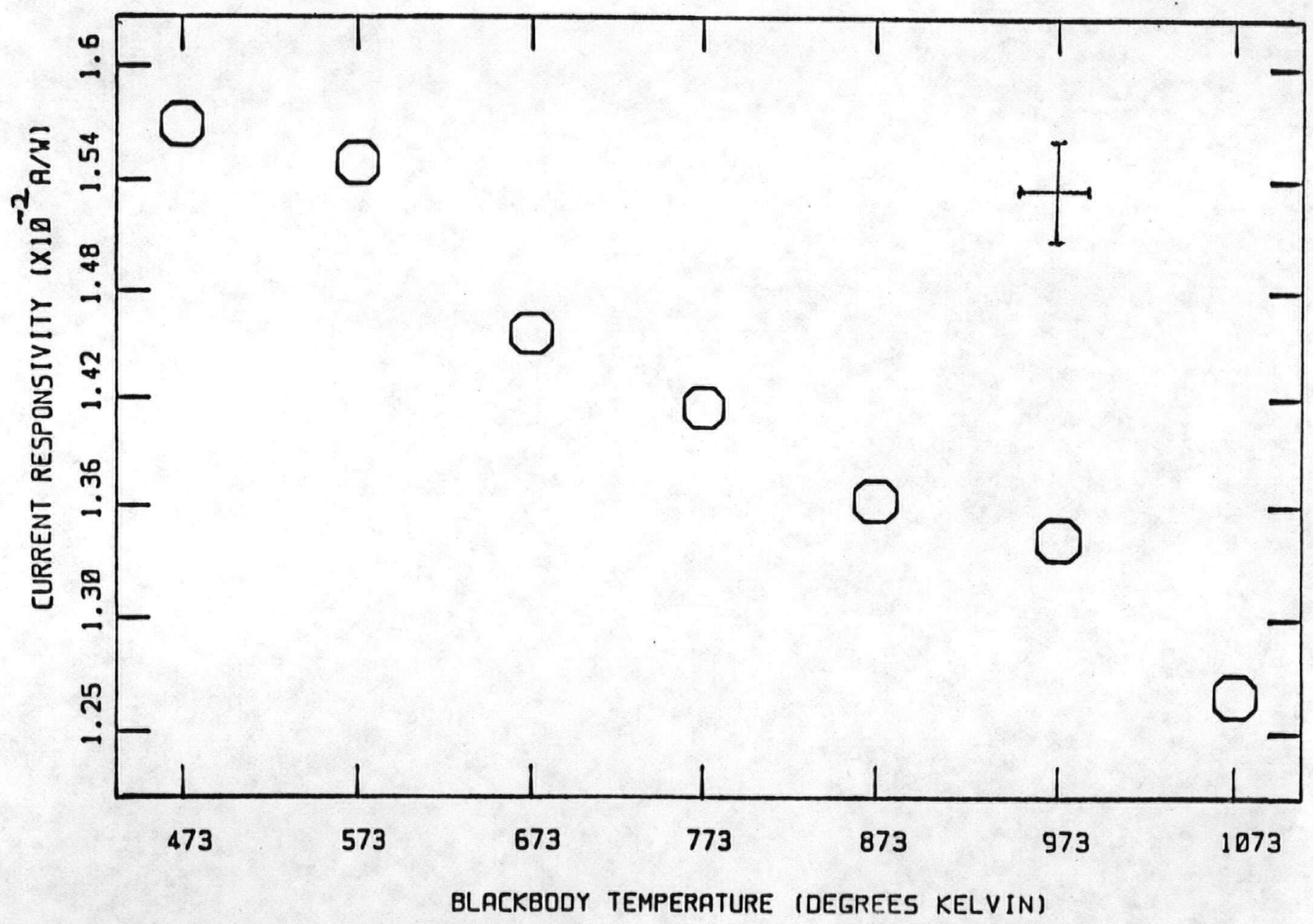

Fig.(4.7) - Current responsivity versus blackbody temperature. 


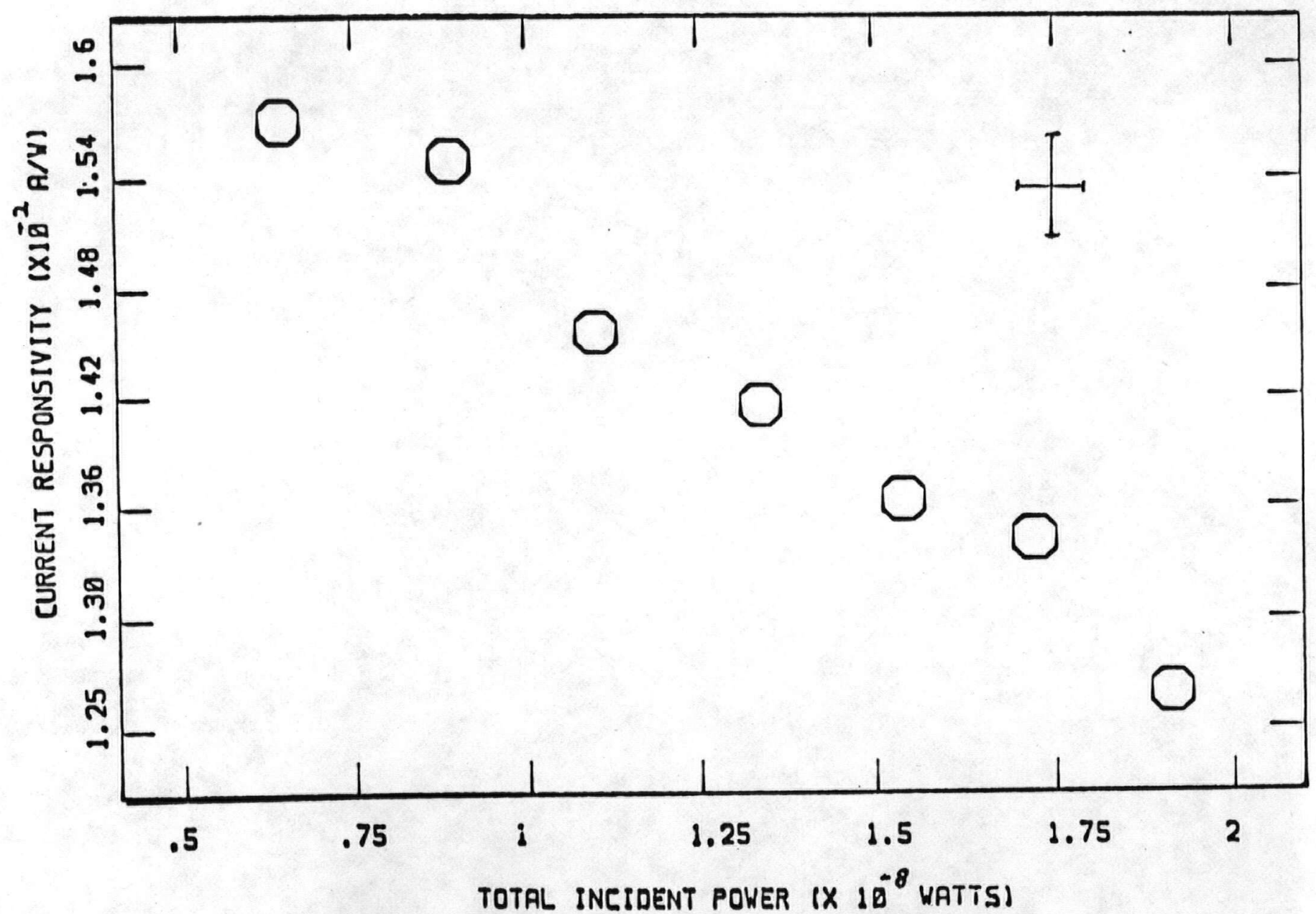

Fig.(4.8) - Current responsivity versus total incident power. 
current measurements could be used to calculate the NEP from the responsivity measurements described above. The value of the resistance of the detector used with a $473^{\circ}$ blackbody was used to determine the proper current noise. The effect due to photon bunching was not considered in this calculation. The current noise was determined to be $\mathrm{I}_{\mathrm{N}}=1.8 \times 10^{-12} \mathrm{~A}$. This results in an NEP $=1.15 \times 10^{-10} \mathrm{~W} / \sqrt{\mathrm{Hz}}$. It is informative to compare this NEP to the background limited NEP given by

$$
N E P_{B L}=\left[\frac{4 P_{B} h v \Delta f}{\eta}\right]^{\frac{1}{2}},
$$

where $P_{B}$ is the background power, $h$ is Planck's constant, $v$ the mean optical frequency, $\Delta f$ is the electrical bandwidth, and $\eta$ is the quantum efficiency. The background power of $4.5 \times 10^{-8} \mathrm{~W}$ results in a background 1 imited NEP of $\mathrm{NEP}_{\mathrm{BL}}=1.1 \times 10^{-12} \mathrm{~W} / \sqrt{\mathrm{Hz}}$. As was expected from the theory presented in chapter 2 , the measured value of the NEP is above that calculated for the theoretical limit. Part of the discrepancy may be due to the extremely high flux level which would lead to a lower responsivity and higher current. There is also some uncertainty associated with the photon flux level. Note that the above analysis is in general qualitative agreement with the theory as presented in chapter 2. 


\section{REFERENCES}

1. Reference 2, Chapter 3, page 6 .

2. Reference 1, Chapter 3, page 99.

3. Reference 1, Chapter 3, page 103, Figure 8.

4. Reference 6, Chapter 2, pp. 136-141.

5. R.D. Hudson, Infrared System Engineering, (Wiley, New York, 1969). 


\section{CHAPTER 5}

SUMMARY AND CONCLUSIONS

This thesis has presented a complete theory of the generationrecombination noise associated with extrinsic photoconductive detectors. The statistical approach of past treatments has been employed in greater generality and scope. Our treatment has allowed for both the thermal and photo-excitation of charge carriers. The analysis also accounted for the impurity compensation effect on detector response characteristics. The method of detailed balancing, and Master equation techniques were used to derive closed form equations of several detector response characteristics.

Chapters 3 and 4 described a Ge:Ga detection system. The experimental setup and system optimization were discussed in detail. Experimental measurements of the detector's response characteristics were found to be in good qualitative agreement with the theory developed in chapter 2 . 


\title{
GENERATION-RECOMBINATION NOISE IN EXTRINSIC \\ PHOTOCONDUCTIVE DETECTORS
}

\author{
by
}

Thomas John Brukilacchio

\author{
Submitted in Partial Fulfillment \\ of the \\ Requirements for the Degree \\ MASTER OF SCIENCE
}

Supervised by Professor Robert W. Boyd

The Institute of Optics

University of Rochester

Rochester, New York

1983 


\section{ACKNOWLEDGEMENTS}

I wish to express my gratitude to Professor Robert W. Boyd for his continued support and guidance throughout my undergraduate and graduate studies. I also wish to thank the other members of the Quantum Electronics Group for their support throughout this research. Mark Skeldon's insight in the theory section and Steven Wilk's assistance with the cryostat and vacuum system are also acknowledged. The advice on infrared detection and the loan of equipment from Professor Judith Pipher of the Department of Physics and Astronomy is also highly appreciated.

This work was funded by NASA through a research program of the Jet Propulsion Laboratory, California Institute of Technology. I thank Donald Langford of Jet Propulsion Laboratory for providing a design for the preamplifier, and also for the donation of the detectors and feedback resistors.

I am especially thankful for the warmth and comradery of my close friends in and out of the University of Rochester community. Finally, I wish to convey my love and respect toward my family for their returned love and moral support throughout my college experiences. 


\section{CURRICULUM VITAE}

The author was born on December 11, 1959 in Newport, New York. He graduated in 1978 from West Canada Valley Central School in Newport, New York, and received a Bachelor of Science degree in Optics from the University of Rochester in May of 1982. The author began his graduate research at the Institute of Optics of the University of Rochester in September of 1982. His work was supported through a research grant from Jet Propulsion Laboratory. The author's research was supervised by Dr. Robert $W$. Boyd. 
ABSTRACT

A complete theory of generation-recombination noise is developed for extrinsic photoconductive detectors. A statistical approach uses Master equation techniques to derive closed form equations relating the noise and response characteristics of extrinsic detectors. Specifically, this treatment provides equations for the mean and mean-square fluctuation in the number of carriers, the response time, mean carrier lifetime, responsivity, and the noise equivalent power. The theory accounts for both the thermal and photo-induced generation of carriers. Impurity compensation of the majority carrier is addressed in this theory and is shown to have a significant effect on the noise and the response characteristics of this class of detectors.

A Ge:Ga far infrared detection system is designed, built, and tested. Responsivity measurements are made in the presense and absense of background photon flux. Dark current noise measurements are taken as a function of the detector temperature. The noise and response characteristics of the detector are compared to the theory developed in this thesis and are shown to be in good agreement with this theory. 
LIST OF FIGURES

FIGURE

PAGE

2.1 Photoconductor Impurity Levels.

Impurity Compensation of Photoconductive Materials.

2.3

The Ionization of $\mathrm{N}$ Free Electrons.

2.4

Fractional ionization as a function of the generation rate.

2.5

Thermal generation rate as a function of the dimensionless temperature.

2.6

Fractional ionization versus dimensionless temperature at the limits of the compensation ratio.

2.7

Fractional ionization versus dimension-

less temperature for factors of $\Gamma_{0}$ at $\mathrm{N}_{\mathrm{a}} / \mathrm{N}_{\mathrm{d}}=0$.

2.8 Mean-square carrier fluctuation versus

fractional ionization.

2.9 Mean-square carrier fluctuation versus generation rate.

2.10 Mean-square carrier fluctuation versus dimensionless temperature.

2.11 Modified mean-square carrier fluctuation versus fractional ionization. It. represents the degree to which the statistics are Poissonian.

versus generation rate. 
2.13 Modified mean-square carrier fluctuation 30 versus dimensionless temperature.

2.14 Normalized response time versus fractional ionization.

2.15 Normalized response time versus generation rate.

2.16

Normalized response time versus dimensionless temperaure.

Normalized mean carrier lifetime versus fractional ionization

2.18

Normalized mean carrier lifetime versus generation rate.

Normalized mean carrier lifetime versus dimensionless temperature.

Normalized responsivity versusfractional ionization.

Normalized responsivity versus generation rate.

$34-b$

Normalized responsivity versus dimensionless temperature.

2.23 Dimensionless NEP versus fractional ionization. rate.

Dimensionless NEP versus dimensionless temperature.

3.1 Experimental Setup.

3.2 Relative Wavelength Response of Ge:Ga Detector.

3.3 Impurity levels in Ge:Ga at 2.5 degrees Kelvin.

3.4 Detector mount and integrating cavity. 

gain of 100 .

Fractional ionization versus temperature.

Dark current versus temperature. temperature.

4.6 Photocurrent versus total incicent power.

4.7 Current responsivity versus blackbody temperature.

4.8 Current responsivity versus total 
TABLE OF CONTENTS

CURRICULUM VITAE

Page

ACKNOWLEDGEMENTS

ii

ABSTRACT

iii

ABSTRACT

iv

LIST OF FIGURES

vii

CHAPTER

1 INTRODUCTION . 1

2 THEORY OF GENERATION-RECOMBINATION NOISE 4

2.1. Introduction

2.2 A Statistical Treatment of Generation-Recombination Noise

2.3 The Fractional Ionization of Available Donor Impurities

2.4. Graphical Representation of Detector Properties

2.5 Conclusion

3 EXPERIMENTAL SETUP OF A Ge;Ga DETECTION SYSTEM

3.1 Introduction $\quad 49$

3.2 The Ge:Ga Detector 49

3.3 Helium Dewar 55

3.4 Detector Electronics 58

3.5 Filter and Window Materials 66

4 EXPERIMENTAL RESULTS $\quad 70$

4.1 Introduction 70

4.2 Initial Testing and System
Optimization 
4.3 Experimental Results

5 SUMMARY AND CONCLUSIONS 


\section{CHAPTER 1}

\section{INTRODUCTION}

Generation-recombination noise constitutes the dominant noise mechanism in semiconductors and in photoconductive materials. This noise arises from the statistical fluctuation in the number of conduction-band electrons and valence-band holes that are available to conduct a current at any given time. Recent interest in highly sensitive photoconductive detectors has motivated the investigation of generation-recombination $(g-r)$ noise.

Pioneering groundwork that led to a treatment of $g-r$ noise in semiconductors was carried out by K.M. van Vliet ${ }^{1}$ and by R.E. Burgess $^{2}$ in the mid $1950^{\prime} \mathrm{s}$. Burgess showed that equivalent results could be derived from thermodynamic and statistical approaches to the fluctuation in the number of charge carriers in a semiconductor.

This thesis presents a complete theory of the generationrecombination noise associated with extrinsic photoconductive detectors. To the knowledge of the author, this thesis represents the most complete treatment of $\mathrm{g}-\mathrm{r}$ noise available in literature to date. Master equation techniques are employed in deriving closed form equa- 
tions for the bulk response characteristics of extrinsic photoconductive detectors. This theory accounts for both the thermal and photo-induced generation of charge carriers. Additionally, we address the effects on the response characteristics of a detector due to the impurity compensation of the majority carrier. The detector response functions plotted in chapter 2 indicate that impurity compensation can lead to dramatic differences in the response characteristics of extrinsic photoconductive detectors.

Within the scientific community, astronomers may benefit the most from a more complete understanding of $g-r$ noise theory, as they often observe under conditions of very low backgrounds. Recent astronomical interest in the space-based applications of 100 micron detection led to the availability of Ge:Ga detectors to the author. Chapters 3 and 4 of this thesis describe a 100 micron Ge:Ga detection system constructed by the author. Detector response measurements were found to be in good qualitative agreement with the theory of generation-recombination noise as presented in chapter 2. 


\section{REFERENCES}

1. K.M. van Vliet, Noise in Semiconductors and Photoconductors, (IRE, March 3, 1958).

2. R.E.Burgess, Fluctuations of the Numbers of Electrons and Holes in a Semiconductor, (Proceedings of the Physical Society of London, B 68, 1955). 
CHAPTER 2

THEORY OF GENERATION-RECOMBINATION NOISE

\subsection{Introduction}

This chapter presents a theory that is capable of producing explicit expressions for the statistical fluctuations in the number of charge carriers in a given volume of a photoconductive material. This theory takes proper account of the presence of both donor and acceptor impurities. A method of detailed balancing of the transition rates of electrons is employed in steady state to determine specific characteristics of photoconductive materials.

Both Burgess ${ }^{1}$ and van vilet ${ }^{2}$ have presented fairly comprehensive treatments of fluctuations in impurity doped semiconductors, but neither takes proper account of the effect of compensating impurities on the response characteristics of the detector. As will be shown below, the effect of impurity compensation is often far from negligible.

Two alternate formulations of generation-recombination theory have been treated by the aforementioned authors: a statistical 
approach and a thermodynamic approach. The present analysis will follow the statistical approach, because this approach allows us to treat a system that is in the steady state, but not necessarily in thermodynamic equilibrium. Thermodynamic arguments will be introduced only to account for the rate at which carriers are generated by thermal processes. The present model should thus be well suited to the treatment of radiation detectors.

Before developing the theory, it is instructive to illustrate the processes that take place in an impurity-compensated photoconductive detector by means of energy band diagrams. We must point out that gallium is an acceptor impurity in Ge:Ga, the detector material used in the experimental work also reported in this thesis. The following analysis, however, will refer to an n-type photoconductor because it is easier to conceptualize the motion of electrons than that of holes. This convention is commonly employed in the literature on this subject. In the ensuing discussion we will assume that the detector temperature is low enough that band to band transitions can be ignored.

Figure (2.1) depicts an impurity photoconductor containing a total of $\mathrm{N}_{d}$ donor impurity sites lying close to the bottom of the conduction band and also containing a total of $\mathrm{N}_{\mathrm{a}}$ acceptor impurity sites lying within the bandgap, close to the top of the valance band. Since we are considering an n-type photoconductor, the total number of donor impurities is greater than the total number of acceptor impurities. 
CB

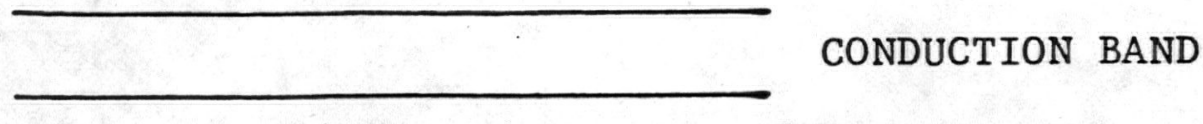

co-c-a. $\quad \mathrm{N}_{\mathrm{d}}$ DONOR INPURITIES

O-

$\mathrm{VB}$

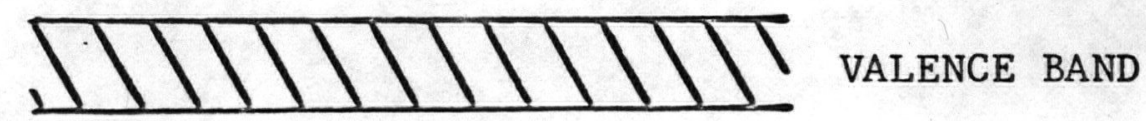

Fig(2.1) - Photoconductor Inpurity Levels

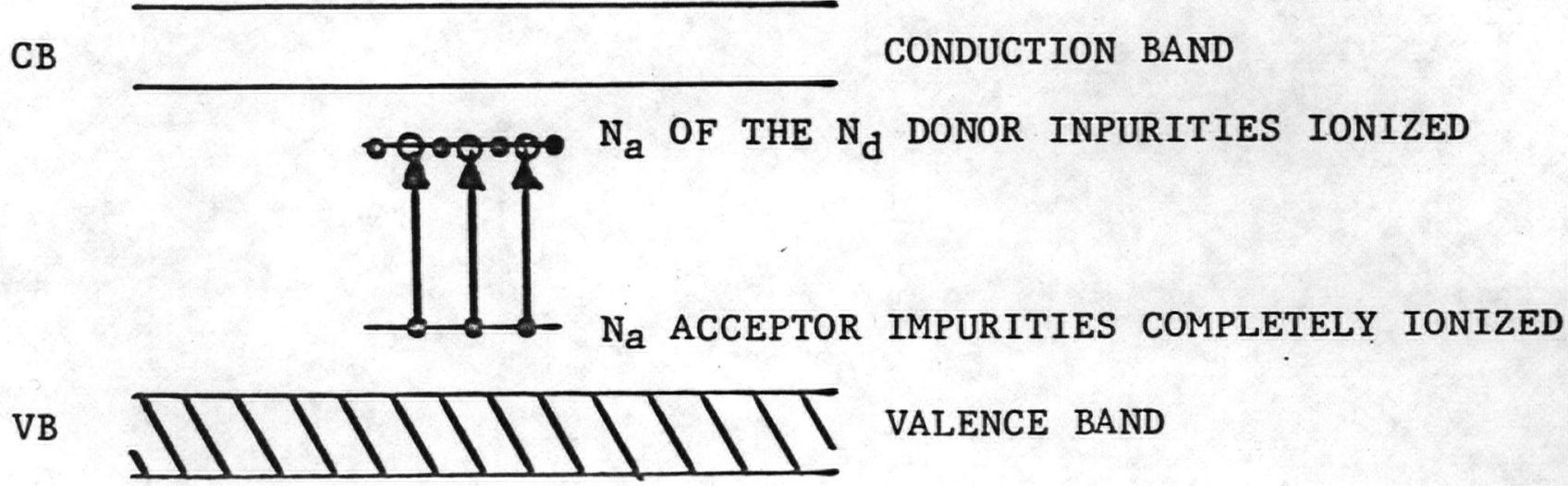

Fig(2.2) - Impurity Compensation of Photoconductive Materials 
Figure (2.2) illustrates that $\mathrm{N}_{\mathrm{a}}$ of the $\mathrm{N}_{\mathrm{d}}$ donor impurities are ionized even at the temperature of absolute zero. This phenomenon is known as compensation since only $\mathrm{N}_{d}-\mathrm{N}_{a}$ of the donor impurities are available for optical excitation to the conduction band.

As illustrated by Figure (2.3), we let $\mathrm{N}$ denote the total number of free electrons within the conduction band. Hence $\mathrm{N}_{a}+\mathrm{N}$ of the $\mathrm{N}_{d}$ donor impurities are ionized and therefore available as recombination centers. Likewise, $N_{d}-N_{a}-N$ of the $N_{d}$ donor impurities are un-ionized and available for excitation to the conduction band. Electrons can be excited from the donor levels to the conduction band through both thermal-excitation and photo-excitation.

\subsection{A Statistical Treatment of Generation-Recombination Noise}

Generation-recombination noise results from the time varying statistical fluctuation in the number of free (i.e., conduction band) charge carriers. ${ }^{3}$ These statistical fluctuations can be calculated from the transition rates of electrons between the conduction band and the donor impurity level. The present analysis considers only one independently fluctuating variable, in this case the number of conduction band electrons $N$. The validity of this assumption has been illustrated in figure (2.2). Thus, we are considering a two level system in that only two of the levels of figure (2.2) interact dynamically with the optical radiation.

The method of detailed balancing of the generation and recombination rates is applied to determine the statistical fluctuation in 
CB

N FREE ELECTRONS

$\mathrm{N}_{\mathrm{d}}$ DONOR IMPURITIES

$$
\begin{aligned}
& \mathrm{N}_{\mathrm{a}}+\mathrm{N} \text { ionized } \\
& \mathrm{N}_{\mathrm{d}}-\left(\mathrm{N}_{\mathrm{a}}+\mathrm{N}\right) \text { un-ionized }
\end{aligned}
$$

ALL $\mathrm{N}_{\mathrm{a}}$ ACCEPTORS IONIZED

VB

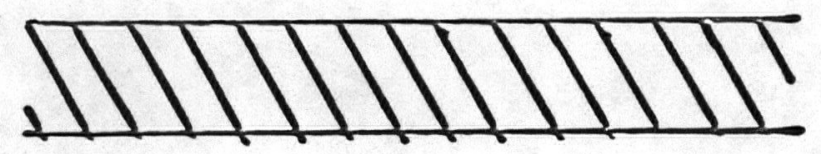

Fig(2.3) - The Ionization of $\mathrm{N}$ Free Electrons 
the number of conduction band electrons, as well as the mean-square carrier fluctuation, response time, mean carrier lifetime, and the mean-square g- $r$ current noise (henceforth $g-r$ will denote generationrecombination).

The transition probabilities per unit time of electrons between the donor level and the conduction band are defined as follows: $\mathrm{g}(\mathrm{N}) \mathrm{dt}$ is the probability that one additional electron is excited to the conduction band in a time $\mathrm{dt}$ if $\mathrm{N}$ electrons are already there. The generation rate $g(N)$ is assumed to be proportional to the product of the number of un-ionized donors $\mathrm{N}_{d}-\mathrm{N}_{a}-\mathrm{N}$ with the generation coefficient $\gamma$ as follows:

$$
g(N)=\gamma\left(N_{d}-N_{a}-N\right) .
$$

The generation coefficient $\gamma$ is assumed to be the sum of a thermal contribution that increases rapidly with temperature and a radiative contribution that increases linearly with photon flux. Similarly, a recombination rate $r(N)$ is defined such that $r(N)$ dt is the probability that within a time interval $\mathrm{dt}$ one of the $\mathrm{N}$ electrons initially in the conduction band returns to the donor level. The recombination rate $r(N)$ is assumed to be of the form

$$
r(N)=\rho N\left(N_{a}+N\right),
$$

where $\mathrm{N}_{\mathrm{a}}+\mathrm{N}$ is the number of recombination sites (i.e., the number of ionized donors), and $\rho$ is the recombination coefficient which may be weakly temperature dependent. In his thermodynamic treatment of $g-r$ noise, Burgess ${ }^{4}$ shows that $\rho$ is of the form $\overline{v S} / V$ where $V$ is the 
volume of the sample, $v$ is the thermal velocity of a conduction band electron, and $\bar{S}$ is its mean cross section for capture by a positive ion, the mean being taken over all values of $v$.

It was first shown by Burgess ${ }^{5}$ that master equation techniques can be used to determine the mean number and the mean-square fluctuation in the number of carriers present in a doped semiconductor. His analysis assumes that the functional forms of the generation and recombination rates are known and that they depend on only one fluctuating variable which, as discussed above, we take to be $N$. The master equation describes the time evolution of the probability distribution function $p(N)$, which denotes the probability that the conduction band contains $\mathrm{N}$ electrons. The time rate of change of $p(N)$ is given by [see for instance ref. 6]

$\frac{d}{d t} p(N)=r(N+1) p(N+1)+g(N-1) p(N-1)-p(N)[g(N)+r(N)]$.

Under steady state conditions $\mathrm{dp}(\mathrm{N}) / \mathrm{dt}$ is equal to zero and the equation (2.3) can be solved iteratively to obtain

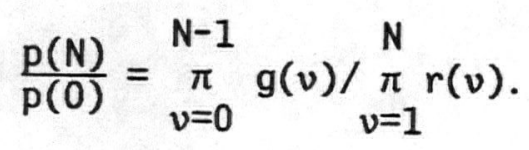

The most probable value $\overline{\mathrm{N}}$ of $\mathrm{N}$ can be found by logarithmic differentiation of $p(N)$ with respect to $N$ and then equating the derivative to zero: 


$$
\frac{d}{d N} \ln p(N)=\frac{\ln g(\bar{N})}{\ln r(\bar{N}+1)}=0
$$

It is assumed that $\overline{\mathrm{N}}$ is large compared to unity. As expected, the equilibrium condition of equation (2.5) leads to equal generation and recombination rates:

$$
g(\bar{N})=r(\bar{N}) .
$$

The variance in $\mathrm{N}$ can be found by expanding $\ln \mathrm{p}(\mathrm{N})$ in a power series centered on $\mathrm{N}=\overline{\mathrm{N}}$ :

$$
\begin{aligned}
\ln p(N)= & \ln p(\bar{N})-\left.(N-\bar{N}) \frac{d}{d N} \ln p(N)\right|_{N=\bar{N}} \\
& +\left.\frac{1}{2}(N-\bar{N})^{2} \frac{d^{2}}{d N^{2}} \ln p(N)\right|_{N=\bar{N}}+\ldots .
\end{aligned}
$$

where the second term will vanish and terms beyond the third can be neglected. Calculation of this last derivative leads to the following gaussian distribution of $\mathrm{N}$ about $\overline{\mathrm{N}}$ :

$$
\begin{aligned}
\left.\frac{d^{2}}{d N^{2}} \ln p(N)\right|_{N=\bar{N}} & =\left.\frac{d}{d N}\left(\frac{d}{d N} \ln p(N)\right)\right|_{N=\bar{N}} \\
& =\left.\frac{d}{d N}(\ln g(N)-\ln r(N))\right|_{N=\bar{N}} \\
& =\frac{g^{\prime}(\bar{N})}{g(\bar{N})}-\frac{r^{\prime}(\bar{N})}{r(\bar{N})},
\end{aligned}
$$


where the prime denotes differentiation with respect to $\mathrm{N}$. Substitution of the gaussian distribution of equation (2.10) into the power series expansion of $\ln p(N)$ of equation (2.7) results in:

$$
\ln p(N)=\ln p(\bar{N})-\frac{1}{2}(N-\bar{N})^{2}\left[\frac{r^{\prime}(\bar{N})}{r(\bar{N})}-\frac{g^{\prime}(\bar{N})}{g(\bar{N})}\right]
$$

or equivalently

$$
p(N)=p(\bar{N}) \exp \left[\frac{-(N-\bar{N})^{2}}{2(\Delta N)^{2}}\right] \text {, }
$$

where the variance of $\mathrm{N}$ is given by

$$
\overline{(\Delta N)^{2}}=\frac{g(\bar{N})}{r^{\prime}(\bar{N})-g^{\prime}(\bar{N})} .
$$

The relevance of the denominator of equation (2.13) is understood by considering the time evolution of the system returning to equilibrium after it has been perturbed by an amount $N-\bar{N}$. The expectation value of the rate of change of $\mathrm{N}$ towards $\overline{\mathrm{N}}$ is

$$
\overline{\frac{d}{d t}(N-\bar{N})}=\frac{\overline{d N}}{d t}=g(N)-r(N),
$$

where for small deviations

$$
\frac{\overline{d N}}{d t} \cong\left[r^{\prime}(\bar{N})-g^{\prime}(\bar{N})\right](N-\bar{N})
$$


Thus, the system relaxes to equilibrium according to

$$
\Delta N(t)=\Delta N(0) \exp \left(-t / \tau^{\prime}\right)
$$

where $\tau^{\prime}$ is the relaxation time given by

$$
\tau^{\prime}=\frac{1}{r^{\prime}(\bar{N})-g^{\prime}(\bar{N})} \text {. }
$$

$\tau^{\prime}$ is interpreted as the response time of the photoconductor or as the incremental carrier lifetime, that is, the lifetime of an additional carrier when $\mathrm{N}$ are known to be already present.

The response time is not to be confused with the mean carrier lifetime $\bar{\tau}$ which is defined as the difference between the mean number of conduction band electrons and the mean number that would exist if the generation rate were zero. Thus, the mean carrier lifetime is given by

$$
\bar{\tau}=\frac{\bar{N}}{g(\bar{N})} .
$$

With the use of equation (2.17), the variance in $\mathrm{N}$ can be expressed as

$$
\overline{(\Delta N)^{2}} \quad=g(\bar{N}) \tau^{\prime} .
$$

This result was first obtained by Burgess ${ }^{8}$ and is known as the $g-r$ theorem.

To arrive at an expression for the mean-square $g-r$ current noise, consider a slab of photoconductive material of cross sectional area $A$ and length $L$ to which a potential of magnitude $V$ has been 
applied. Most generally, the instantaneous value of the current passing through the photoconductive material is related to the total number of free electrons $N(t)=A L n(t)$ and to the total number of free holes $P(t)=A L p(t)$, where $n(t)$ and $p(t)$ are the electron and hole densities respectively, by

$$
i(t)=\frac{e V}{L^{2}}\left[\mu_{n} N(t)+\mu_{p} P(t)\right]
$$

where $\mu_{n}$ and $\mu_{p}$ are the electron and hole carrier mobilities respectively, and $e$ is the fundamental electric charge. The mean value of this current is given by

$$
\bar{i}=\frac{e V}{L^{2}}\left[\mu_{n} \bar{N}+\mu_{p} \bar{P}\right]
$$

The fluctuation in the current $\Delta i=i(t)-\bar{i}$ is thus directly related to the fluctuations in the carrier concentrations $\Delta N=N(t)-\bar{N}$ and $\Delta P=P(t)-\bar{P}$ by

$$
\overline{(\Delta i)^{2}}=\frac{e^{2} v^{2}}{L^{2}}\left[\mu_{n}^{2} \overline{(\Delta N)^{2}}+2 \mu_{n} \mu_{p} \overline{(\Delta N)(\Delta P)}+\mu_{p}^{2} \overline{(\Delta P)^{2}}\right] .
$$

This relation is expressed in terms of $\bar{i}$ and the mobility ratio $b=\mu_{n} / \mu_{p}$ by

$$
\overline{(\Delta i)^{2}}=\overline{i_{g r}^{2}}=\left(\frac{\bar{i}}{b \bar{N}+\bar{P}}\right)^{2}\left[b^{2} \overline{(\Delta N)^{2}}+2 b \overline{(\Delta N)(\Delta P)}+\overline{(\Delta P)^{2}}\right],
$$

where $i_{g r}$ is used to designate the generation-recombination current noise. 9 
The spectral density of the current fluctuations will be given by

$\overline{i_{g r}^{2}}(f)=\left(\frac{\bar{i}}{b \bar{N}+\bar{P}}\right)^{2}\left[b^{2}\left(\overline{(\Delta N)^{2}}(f)+\overline{2(\Delta N)(\Delta P)(f)}+\overline{(\Delta P)^{2}}(f)\right]\right.$.

It can be concluded from equations (2.17) and (2.19) that the correlation function of $\mathrm{N}$ is

$$
C_{N}(t)=g(\bar{N}) \tau^{\prime} \exp \left(-t / \tau^{\prime}\right)
$$

The spectral density of the fluctuation in $\mathrm{N}$ is obtained from the Wiener-Khintchine theorem as

$$
\overline{(\Delta N)^{2}}(f)=\frac{4 g(\bar{N}) \tau^{\prime 2}}{1+\left(2 \pi f \tau^{\prime}\right)^{2}} .
$$

Under the condition that $f \ll 1 / 2 \pi \tau^{\prime}$, the spectral density of $N$ reduces to

$$
\overline{(\Delta N)^{2}}(f)=4 g(\bar{N}) \tau^{\prime 2} .
$$

When an extrinsic photoconductor with a majority of donor impurities is considered, as in the present analysis, $\Delta N \gg \Delta P$ and $\bar{N} \gg \bar{P}$, reducing equation (2.24) to

$$
\overline{i_{g r}^{2}}(f)=\frac{\bar{i}^{2}}{\bar{N}^{2}} \overline{(\Delta N)^{2}}(f) \text {. }
$$

It follows from equation (2.27) that

$$
\overline{i_{g r}^{2}}(f)=\frac{4 \bar{i}^{2}}{\bar{N}^{2}} g(\bar{N}) \tau^{\prime 2},
$$


and from equation (2.19) that

$$
\overline{i_{g r}^{2}}(f)=\frac{4 \bar{i}^{2}}{\bar{N}^{2}} \overline{(\Delta N)^{2}} \tau^{\prime}
$$

Thus, the mean-square $g-r$ current noise is given by the product of the spectral density of the current fluctuations and the electrical bandwidth $\Delta f$ by 10

$$
\overline{i_{g r}^{2}}=4 \frac{\bar{i}^{2}}{\bar{N}^{2}} \overline{(\Delta N)^{2}} \tau^{\prime} \Delta f .
$$

\subsection{The Fractional Ionization of Available Donor Impurities}

An explicit expression for the mean number $\bar{N}$ of conduction band electrons will provide a deeper understanding into detector dynamics. Equating equations (2.1) and (2.2) under conditions of equilibrium and solving fo $\overline{\mathrm{N}}$ results in:

$$
\left.\bar{N}=\frac{-1}{2}\left(\frac{\gamma}{\rho}+N_{a}\right)+\left[\frac{1}{4}\left(\frac{\gamma}{\rho}+N_{a}\right)^{2}+\frac{\gamma}{\rho}\left(N_{d}-N_{a}\right)\right)\right]^{\frac{1}{2}}
$$

It is convenient to describe the properties of the detector in terms of the fractional ionization of available donor impurities. This quantity is given by the ratio of the mean total number of carriers to the total number of available donor impurities as

$$
f=\frac{\bar{N}}{N_{d}-N_{a}}=\frac{\bar{N}}{N_{d}}\left(\frac{1}{1-\lambda}\right),
$$


where

$$
\lambda=\frac{N_{a}}{N_{d}} .
$$

$\lambda$ is referred to as the impurity compensation ratio. Thus, the fractional ionization is given by

$$
f=\left(\frac{1}{1-\lambda}\right)\left\{\frac{-1}{2}\left(\frac{\gamma}{\rho N_{d}}+\lambda\right)+\left[\frac{1}{4}\left(\frac{\gamma}{\rho N_{d}}+\lambda\right)^{2}+\frac{\gamma}{\rho N_{d}}(1-\lambda)\right]^{\frac{1}{2}}\right\}
$$

We define $\gamma / \rho N_{d}$ in equation (2.34) to be the dimensionless generation rate $\Gamma$ :

$$
\Gamma=\frac{\gamma}{\rho N_{d}}
$$

This dimensionless generation rate has both radiative and thermal contributions:

$$
\Gamma=\Gamma_{\text {rad }}+\Gamma_{\text {th }} .
$$

Because the present analysis is a statistical approach, there has been no provision made for how $\Gamma$ scales with detector temperature. R.A. Smith, however, has derived an expression for the thermal generation of carriers through thermodynamic considerations. ${ }^{11}$ His treatment agrees with equation (2.32) when a dimensionless thermal generation rate $\Gamma_{t h}$ is defined as

$$
\Gamma_{\text {th }}=\frac{V}{N_{d}} \frac{1}{2} N_{c} \exp \left(-\varepsilon_{d} / k T\right)
$$


where $V$ is the detector volume, $\varepsilon_{d}$ is the donor energy with respect to the conduction band, $k$ is Boltzmann's constant, and $T$ is the temperature in degrees Kelvin. $N_{C}$ may be interpreted as the effective number of levels per unit volume in the conduction band. If it is assumed that the conduction band is parabolic, $N_{c}$ is given by

$$
N_{c}=2\left(2 \pi m k T / h^{2}\right)^{3 / 2}
$$

where $m$ is the equivalent mass of the electron and $h$ is Planck's constant.

To assure that the present theory is applicable to any system, a dimensionless temperature is defined as

$$
\theta=k T / \varepsilon_{d} .
$$

We also define a material-dependent proportionality constant $\Gamma_{0}$ as

$$
\Gamma_{0}=\frac{V}{N_{d}}\left(\frac{2 \pi m \varepsilon}{h^{2}}\right)^{3 / 2}
$$

Thus, the dimensionless thermal generation rate can be expressed as

$$
\Gamma_{\text {th }}=\Gamma_{0} \theta^{3 / 2} \exp (-1 / \theta)
$$

\subsection{Graphical Representation of Detector Properties}

Much intuition into detector dynamics is gained by representing graphically the quantities derived above as functions of $f, \Gamma$, and $k T / \varepsilon_{d}$. In addition, two figures of merit that are used to evaluate 
the quality of a detector are derived and plotted; the responsivity $R$ and the noise equivalent power (NEP). Those plots that are functions of temperature, with the exception of figure (2.5), are unique to the Ge:Ga detectors described in Chapter 3 of this thesis, taking into account the detectors volume, doping level, and bandgap. All other.plots are general to any extrinsic photoconductive detection system.

First, it is important to understand how the bulk detector properties depend on the operating conditions. Figure (2.4) depicts how the fractional ionization scales with the generation rate as shown by equation (2.34). Note how quickly the fractional ionization saturates toward one. This contrasts to our notion of a two-level system, which would reach a maximum ionization of only one half.

Figure (2.5) illustrates how the thermal generation rate scales with temperature as given by equation (2.41). Notice that the material dependent term $\Gamma_{0}$ has been factored out for generality. Substitution of equation (2.41) into equation (2.34) yields an expression for the fractional ionization as a function of the dimensionless temperature. Figures (2.6) and (2.7) illustrate this functional form. $A \Gamma_{0}=1.33 \times 10^{4}$, which is typical of $\mathrm{Ge}: \mathrm{Ga}$, is used here as well as in the remaining plots in this chapter which are functions of temperature. The temperature for which $k T / \varepsilon_{d}=0.4$ corresponds to approximately 50 degrees Kelvin for the Ge:Ga detector described in Chapter 3. Figure (2.6) illustrates that higher compensation ratios allow for higher operating temperatures. 


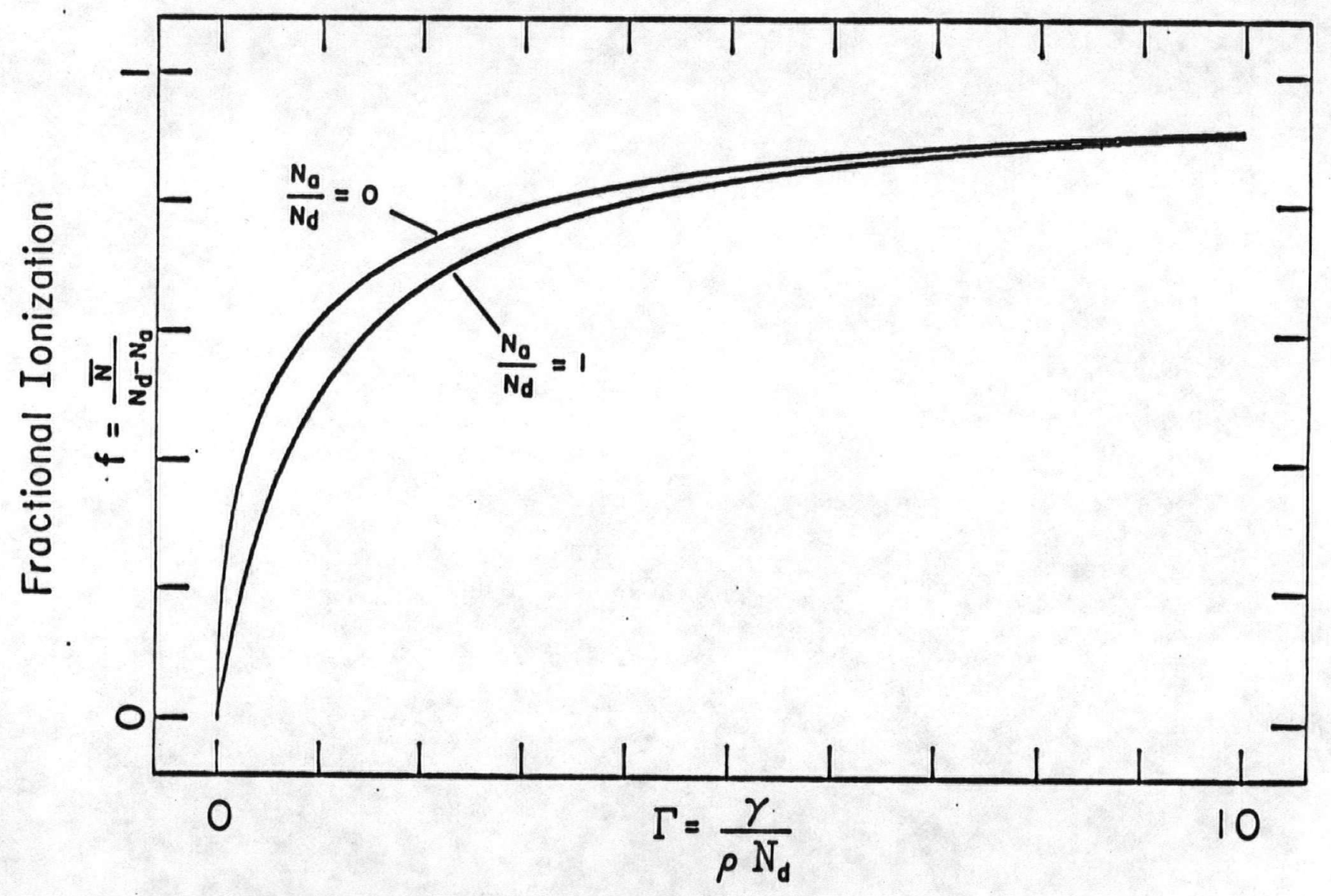

Generation Rate

Fig. (2.4) - Fractional ionization as a function of the generation rate. 


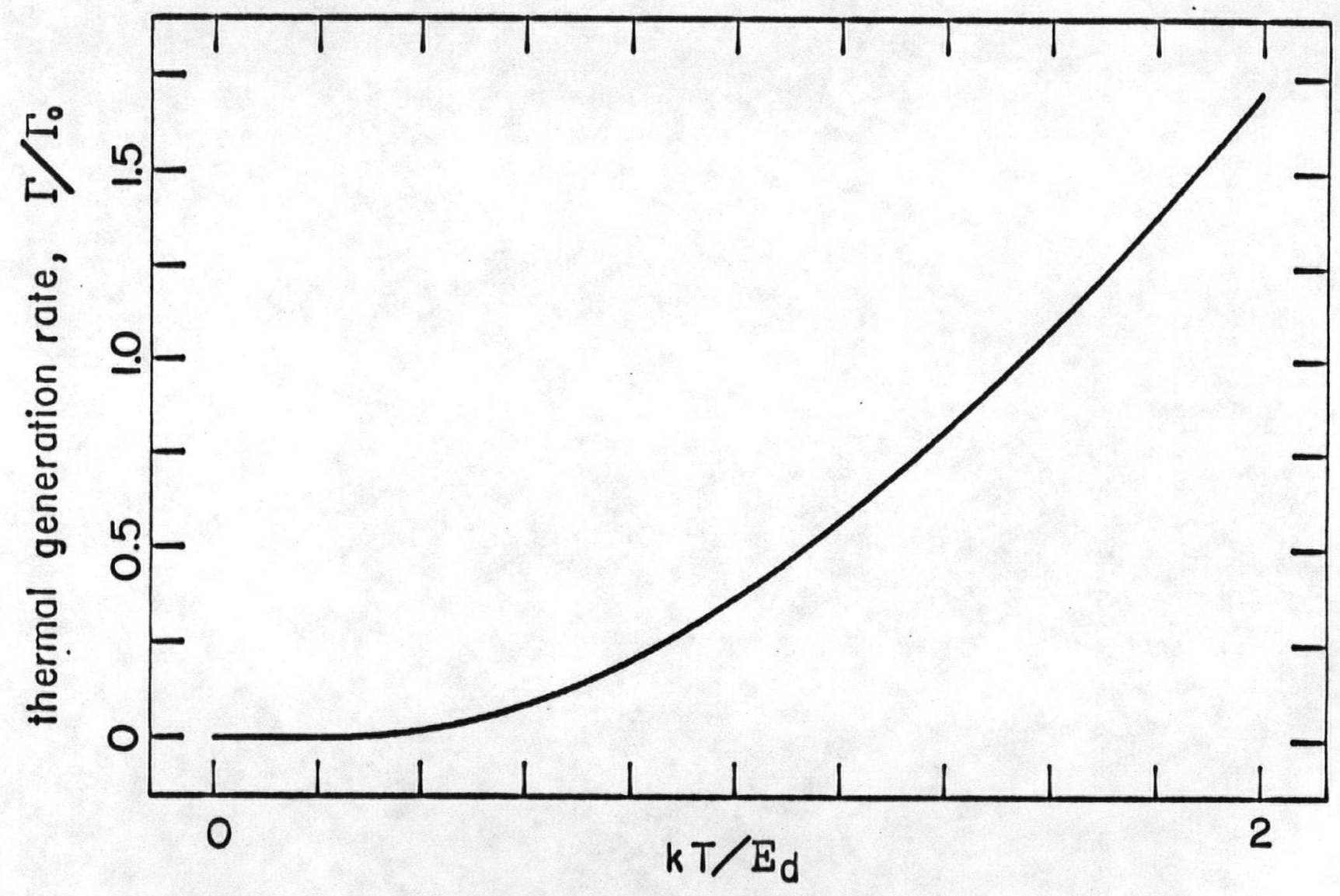

dimensionless temperature

Fig.(2.5) - Thermal generation rate as a function of the dimensionless temperature. 


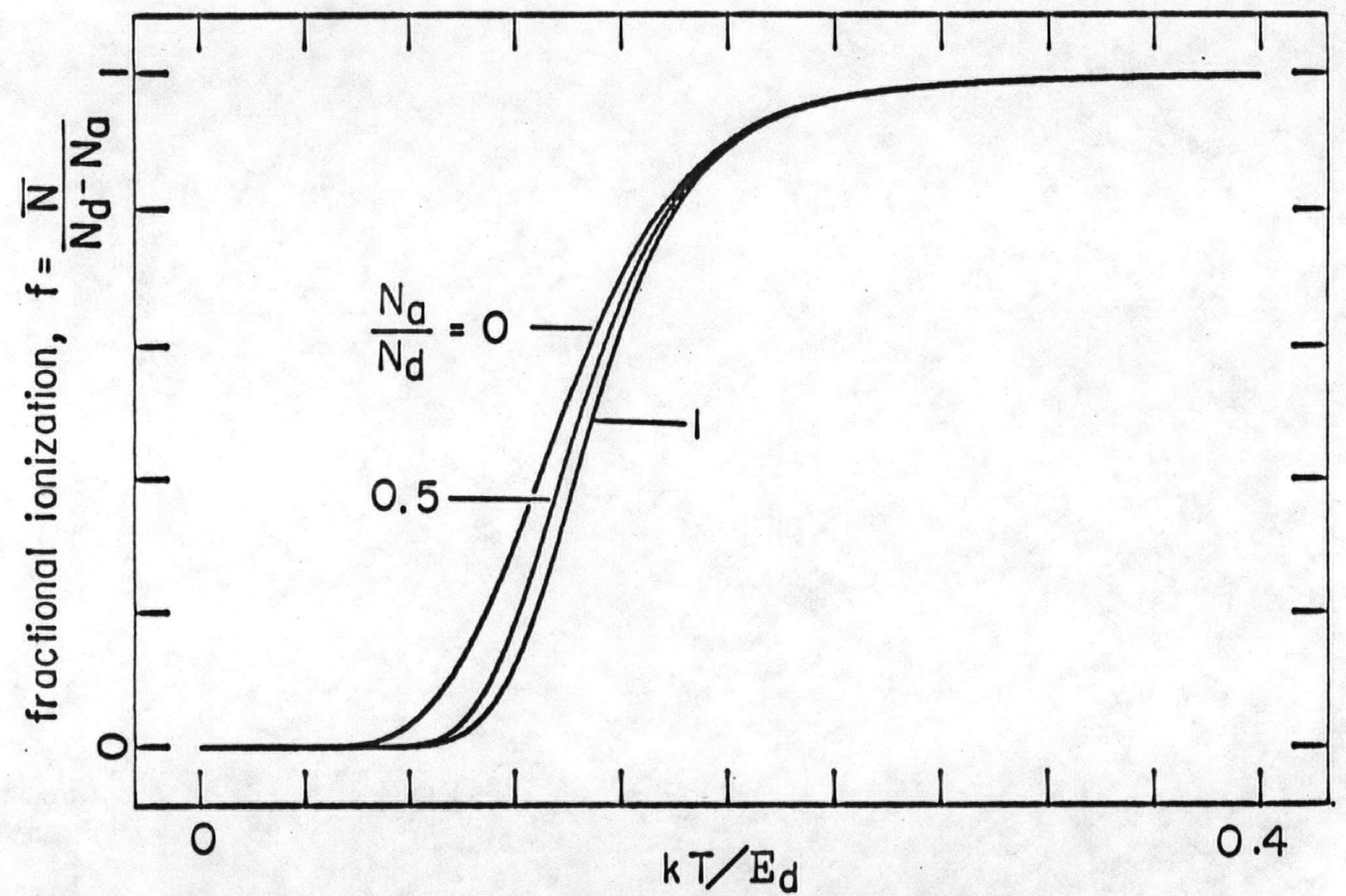

dimensionless temperature

Fig.(2.6) - Fractional ionization versus dimensionless temperature at the limits of the compensation ratio 


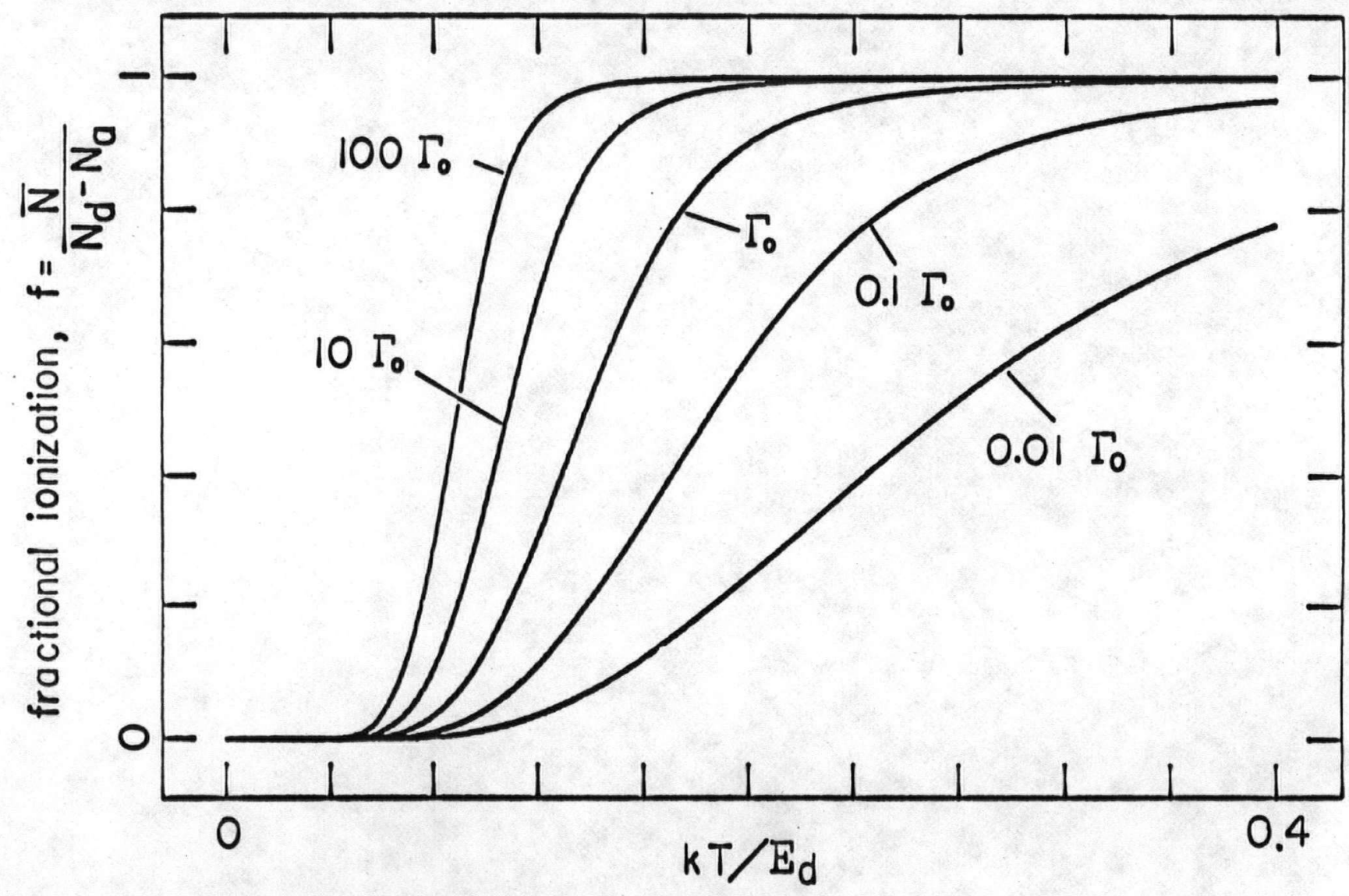

dimensionless temperature

Fig.(2.7) - Fractional ionization versus dimensionless temperature for factors of $\bar{b}$ at $\mathrm{N}_{\mathrm{a}} / \mathrm{N}_{\mathrm{d}}=0$. 
The mean-square carrier fluctuation of equation (2.13) can be expressed in terms of the fractional ionization by substituting the differentiated values of equations (2.1) and (2.2) into equation (2.13) and by expressing it in terms of $f$ by using equation (2.33). This functional form,

$$
\overline{(\Delta N)^{2}}=N_{d} \frac{f(1-\lambda)(1-f)[\lambda+f(1-\lambda)]}{f(2-f)(1-\lambda)+\lambda},
$$

is illustrated in figure (2.8) for various compensation ratios. Figure (2.9) using equation (2.34), and figure (2.10) using equation (2.41), represent the mean-square current noise as functions of the generation rate and dimensionless temperature respectively. As witnessed by figure (2.10), the noise in the Ge:Ga system is appreciable between the temperatures of 4 and 5 degrees Kelvin.

Equation (2.42a) can be modified by dividing by the mean number of carriers $\bar{N}$ and then by expressing $\bar{N}$ in terms of $f$ by using equation (2.33), as

$$
\frac{\overline{(\Delta N)^{2}}}{\bar{N}}=\frac{(1-f)[\lambda+f(1-\lambda)]}{f(2-f)(1-\lambda)+\lambda} .
$$

This function can be interpreted as the degree to which the fluctuations are Poissonian in nature. The value one, corresponds to $100 \%$ correlation to Poissonian statistics. These functions are illustrated in figures (2.11), (2.12), and (2.13).

By differentiating equations (2.1) and (2.2) for the generation and recombination rates at equilibrium and substituting into equation 


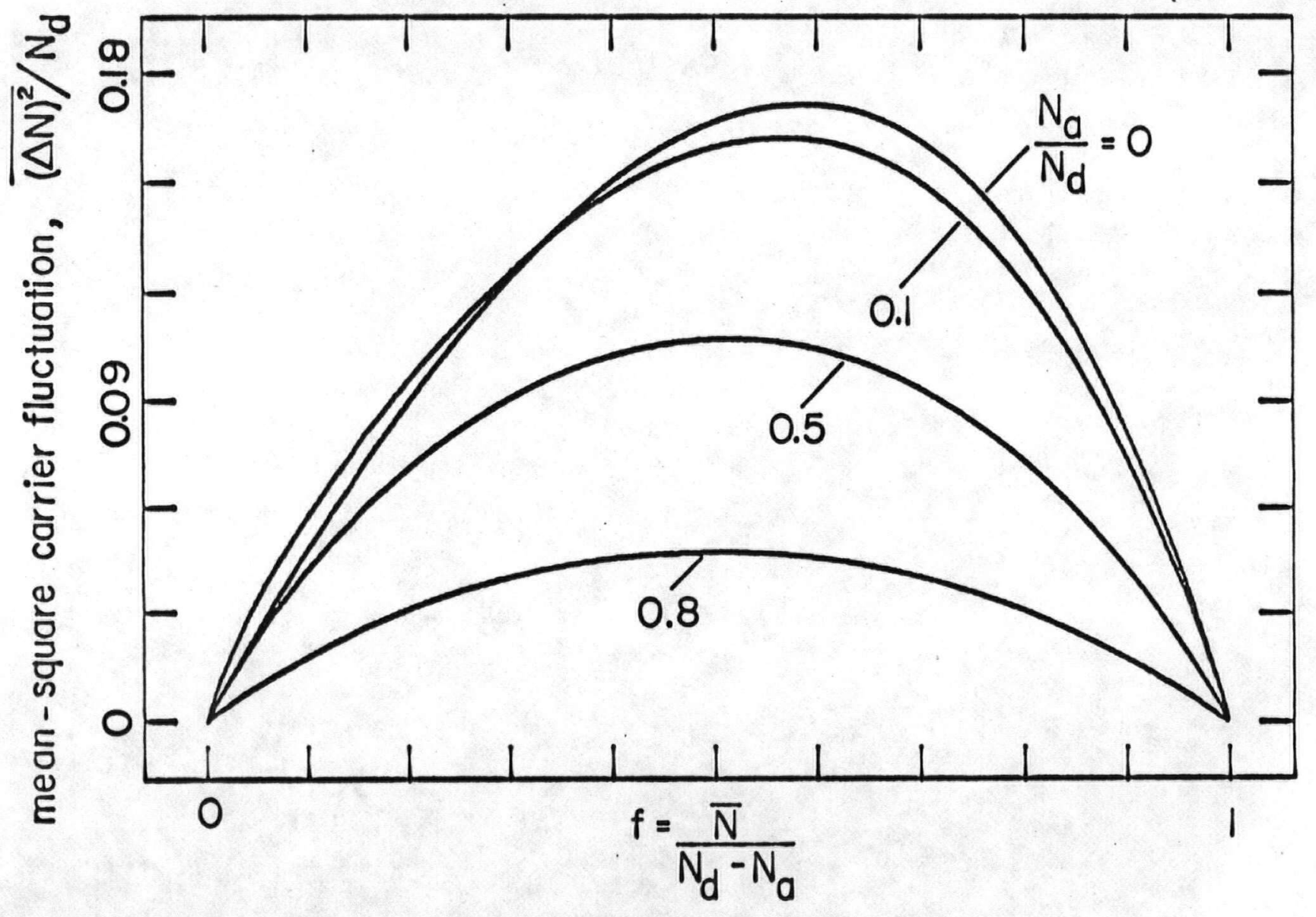

fractional ionization

Fig.(2.8) - Mean-square carrier fluctuation versus fractional ionization. 


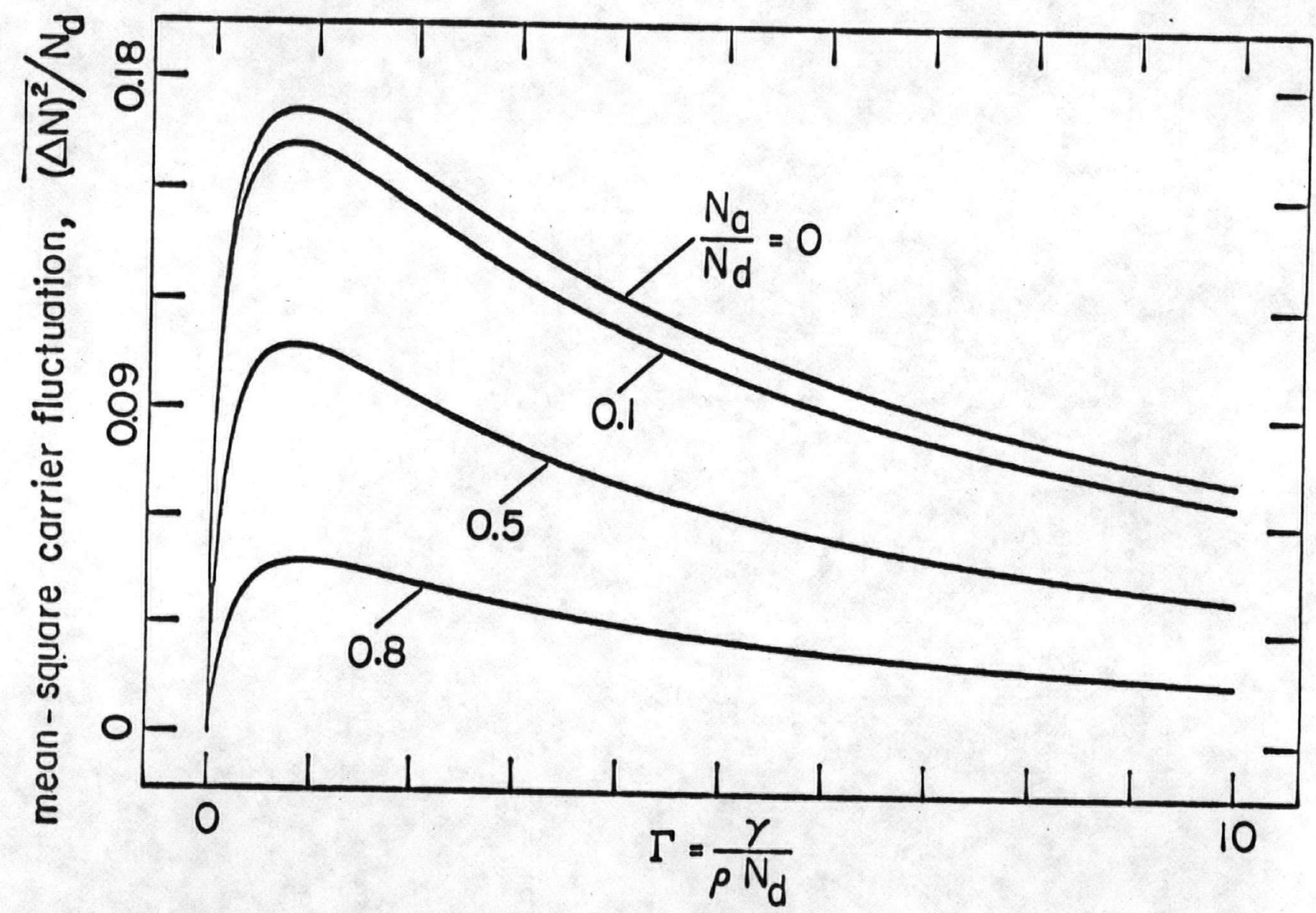

generation rate

Fig.(2.9) - Mean-square carrier fluctuation versus generation rate. 


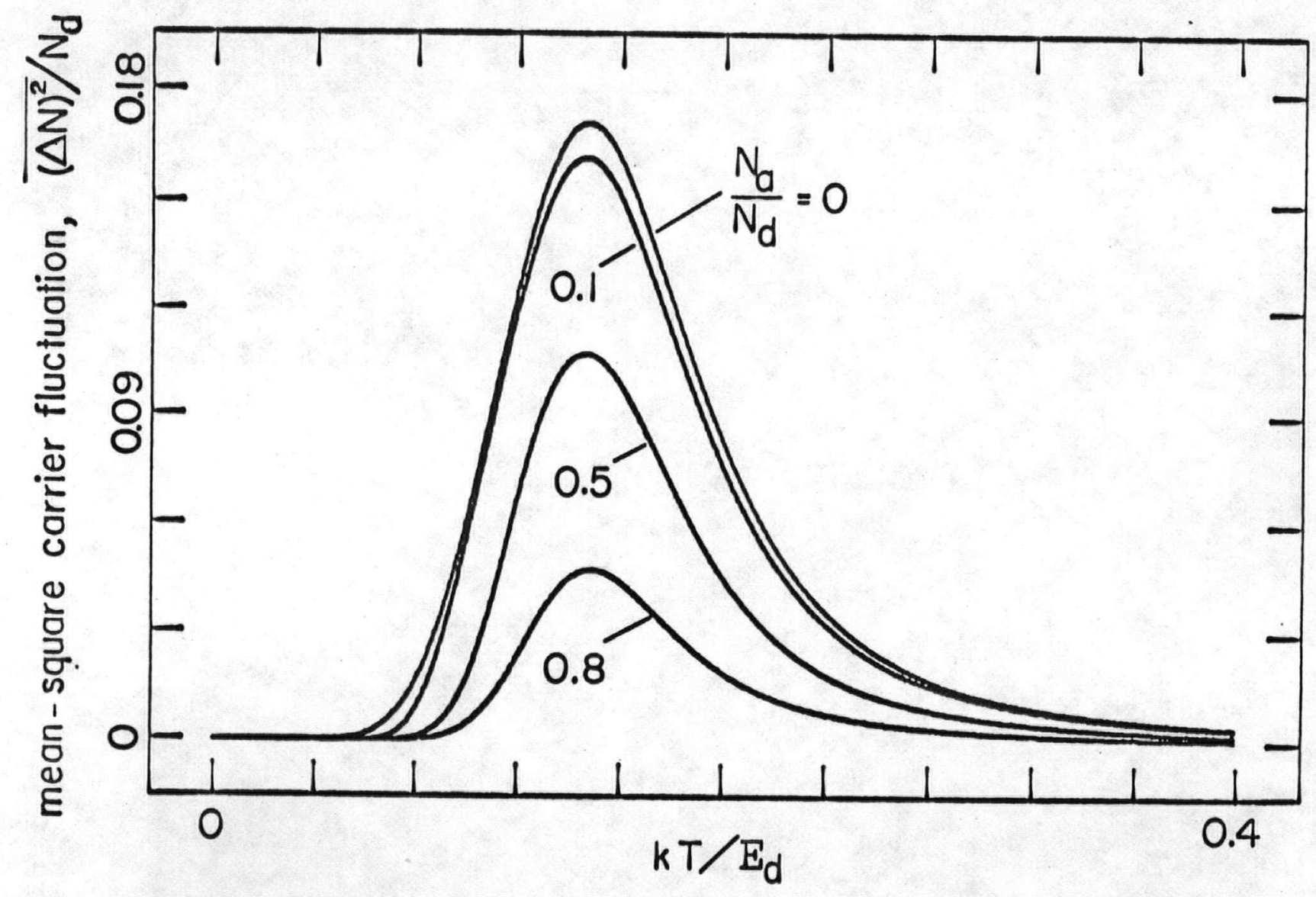

dimensionless temperature

Fig.(2.10) - Mean-square carrier fluctuation versus dimensionless temperature. 


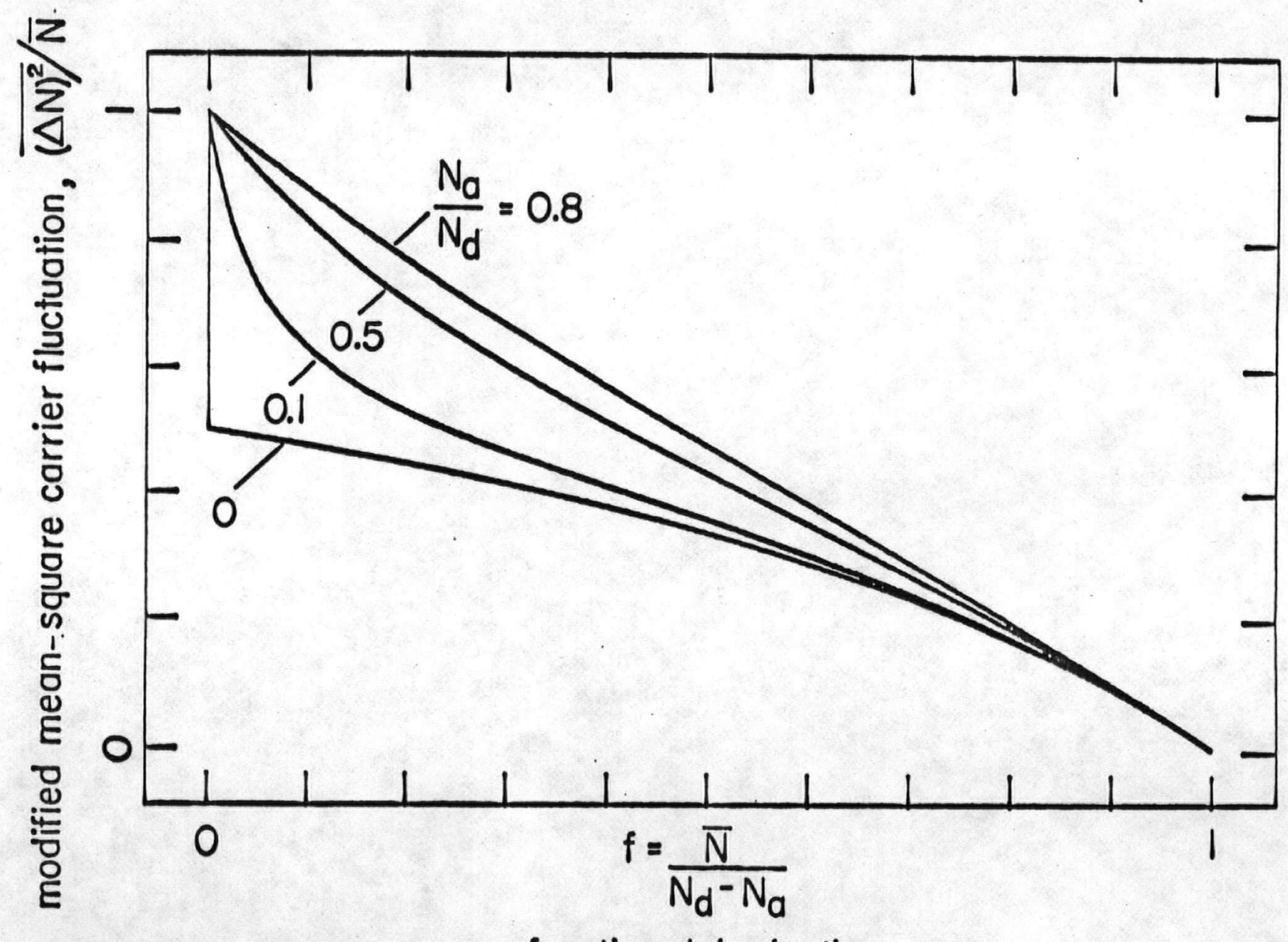

fractional ionization

Fig.(2.11) - Modified mean-square carrier fluctuation versus fractional ionization. It represents the degree to which the statistics are Poissonian. 


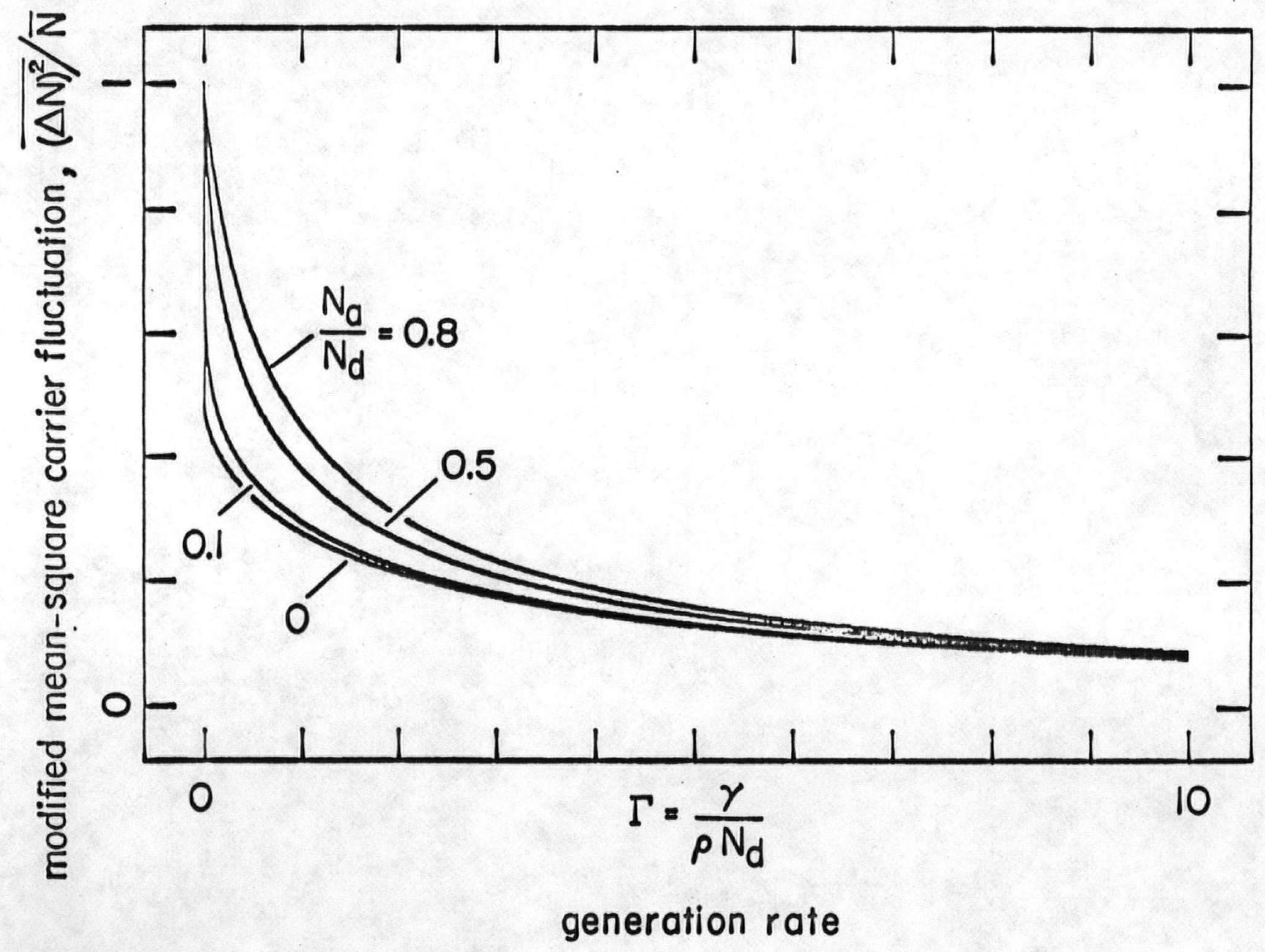

Fig.(2.12) - Modified mean-square carrier fluctuation versus generation rate. 


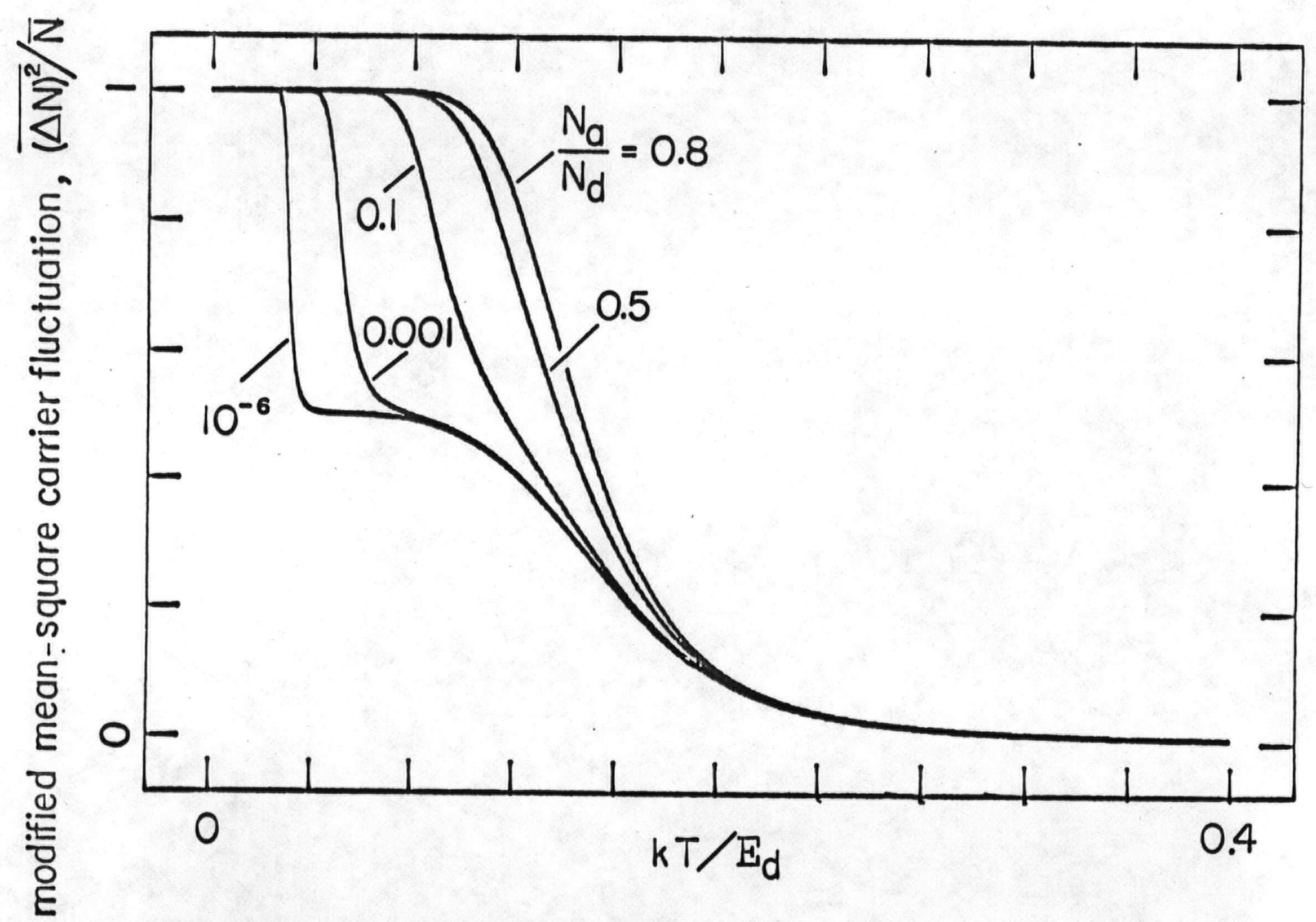

dimensionless temperature

Fig. (2.13) - Modified mean-square carrier fluctuation versus dimensionless
temperature. 
(2.17), an expression for the response time can be found, after further substitution for $f$ and $\lambda$ :

$$
\tau^{\prime}=\frac{1}{\rho N_{d}} \frac{(1-f)}{f(2-f)(1-\lambda)+\lambda} .
$$

Figures (2.14), (2.15), and (2.16) represent the normalized response time as a function of the same three variables $f, \Gamma$, and $k T / \varepsilon_{d}$ respectively. The normalization was necessary in order to show the response times for different compensation levels on the same plot. The mean carrier lifetime is found, by substituting equations (2.1) and (2.32) into equation (2.18), to be

$$
\bar{\tau}=\frac{1}{\rho N_{d}} \frac{1}{\lambda+f(1-\lambda)} \text {. }
$$

The mean carrier lifetime is plotted in figures (2.17), (2.18), and (2.19) as functions of $f, \Gamma$, and $k T / \varepsilon_{d}$ respectively.

The detector responsivity can be derived from the equilibrium g-r condition

$$
\gamma\left(N_{d}-N_{a}-\bar{N}\right)=\rho \bar{N}\left(N_{a}+\bar{N}\right),
$$

by considering the effect on $\bar{N}$ of increasing the generation coefficient by an amount $\delta y$ as follows:

$$
(\gamma+\delta \gamma)\left(N_{d}-N_{a}-\bar{N}-\delta \bar{N}\right)=\rho(\bar{N}+\delta \bar{N})\left(N_{a}+\bar{N}+\delta \bar{N}\right) .
$$

If the assumptions 


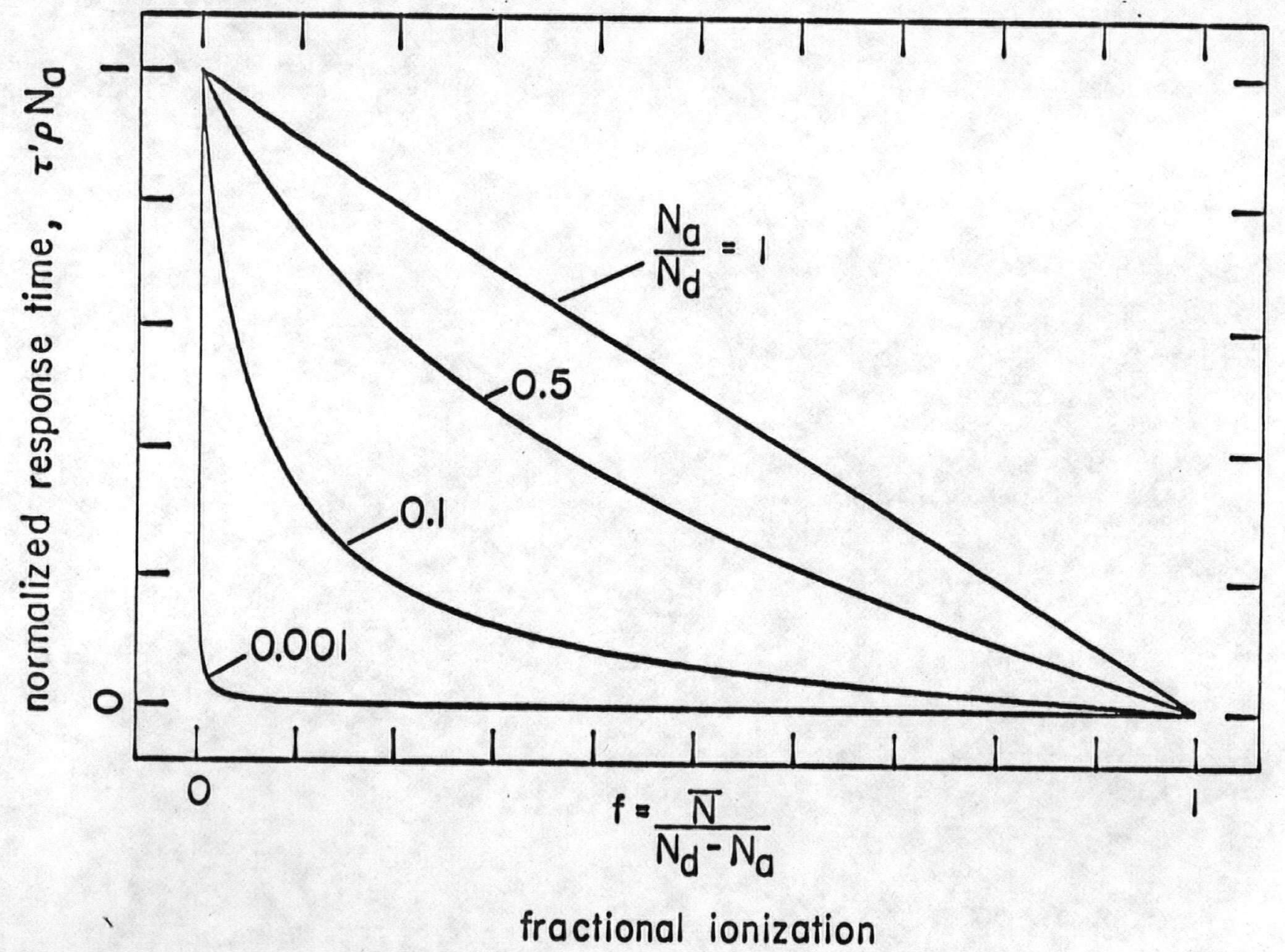

Fig. (2.14) - Normalized response time versus fractional ionization. 


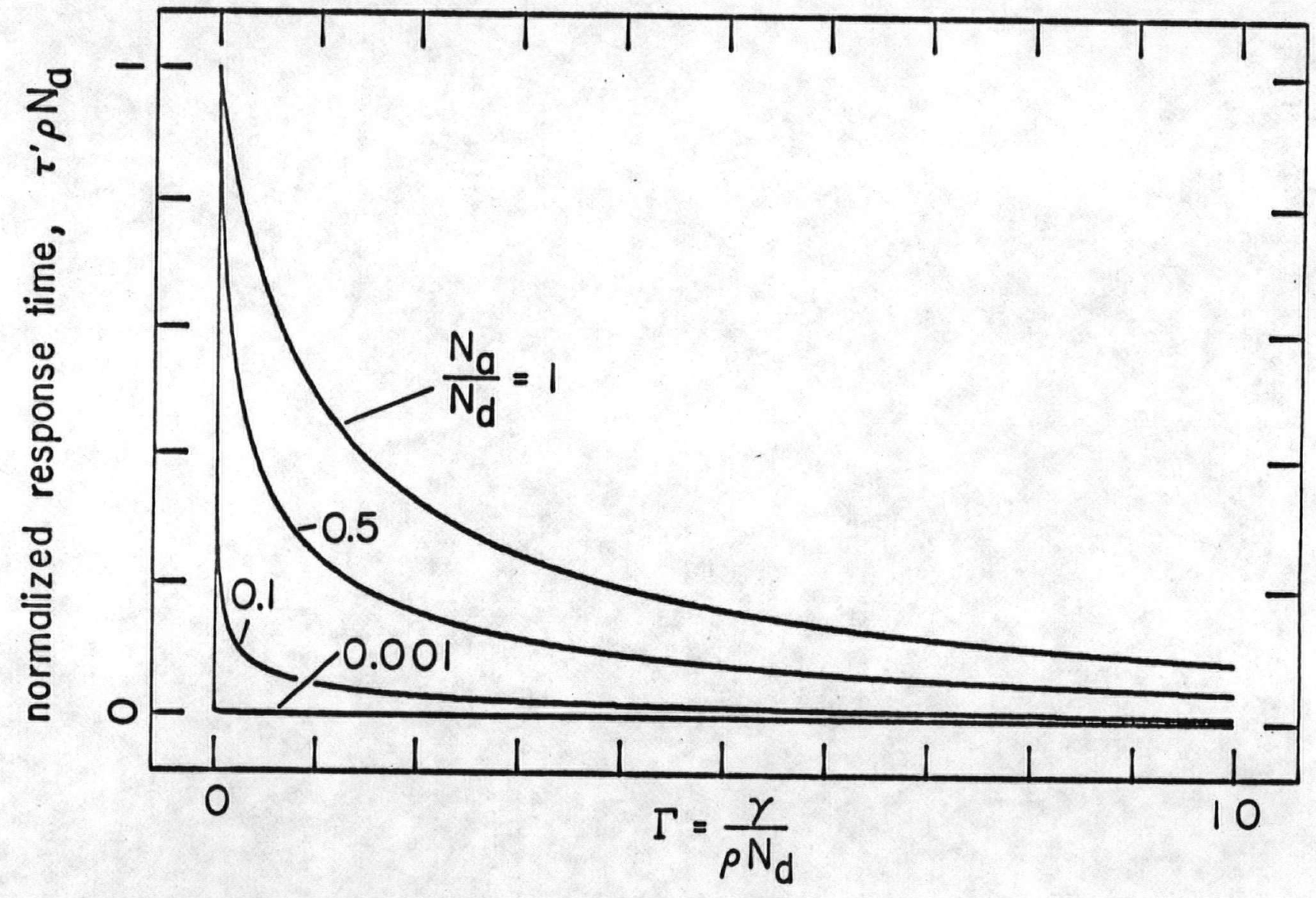

generation rate

Fig.(2.15) - Normalized response time versus generation rate. 


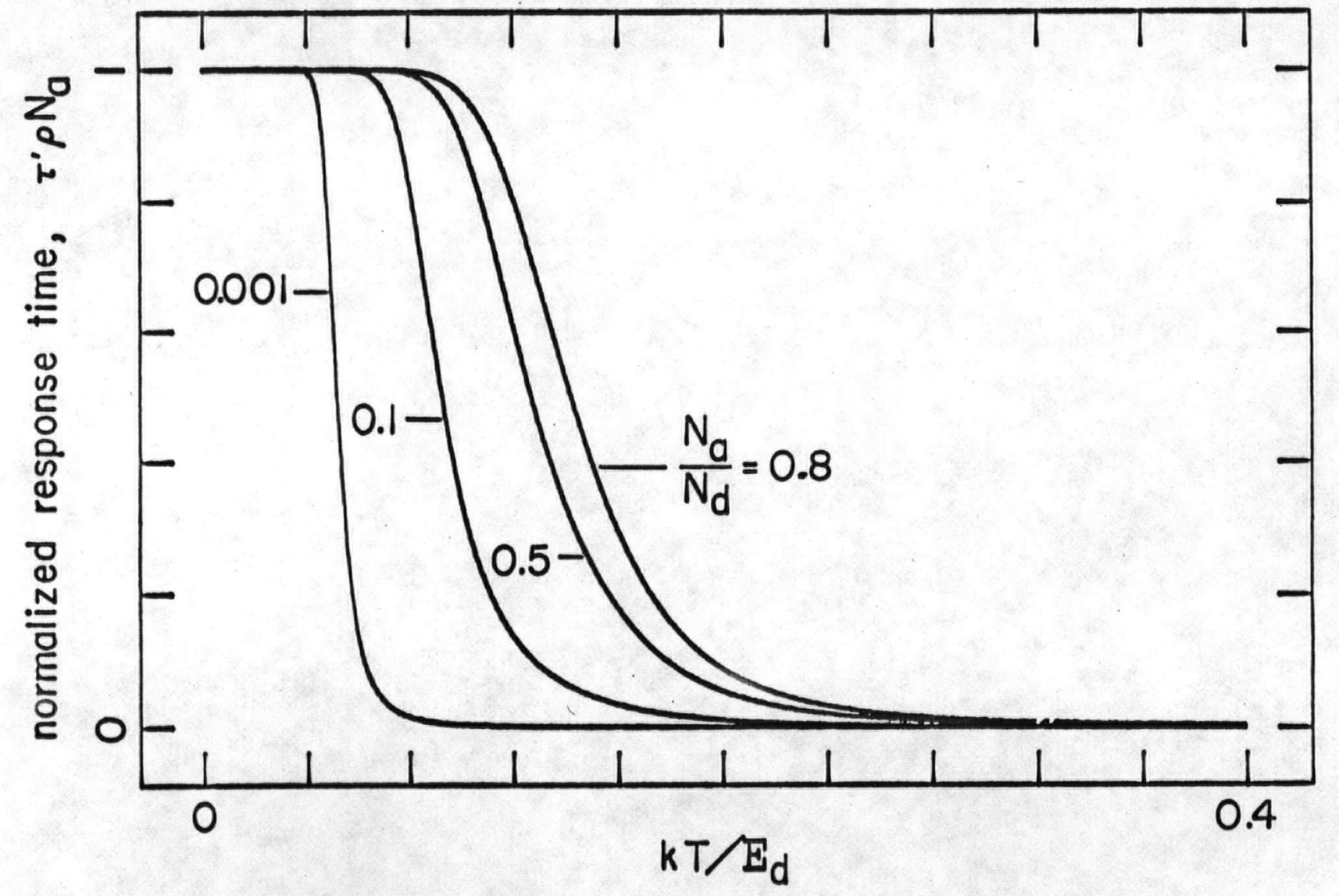

dimensionless temperature

Fig.(2.16) - Normalized response time versus dimensionless temperature. 


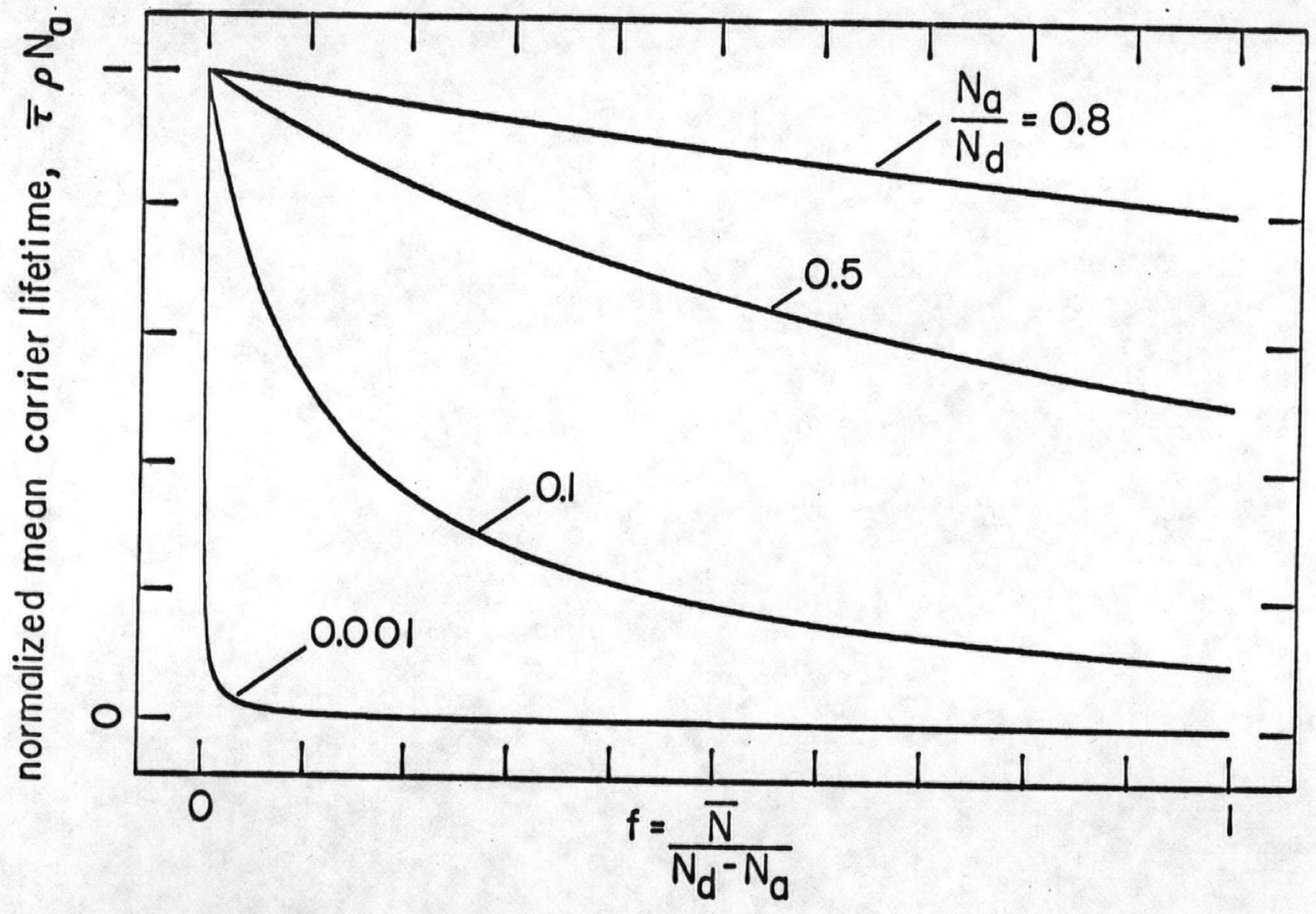

fractional ionization

Fig.(2.17) - Normalized mean earrier lifetime versus fractional ionization. 


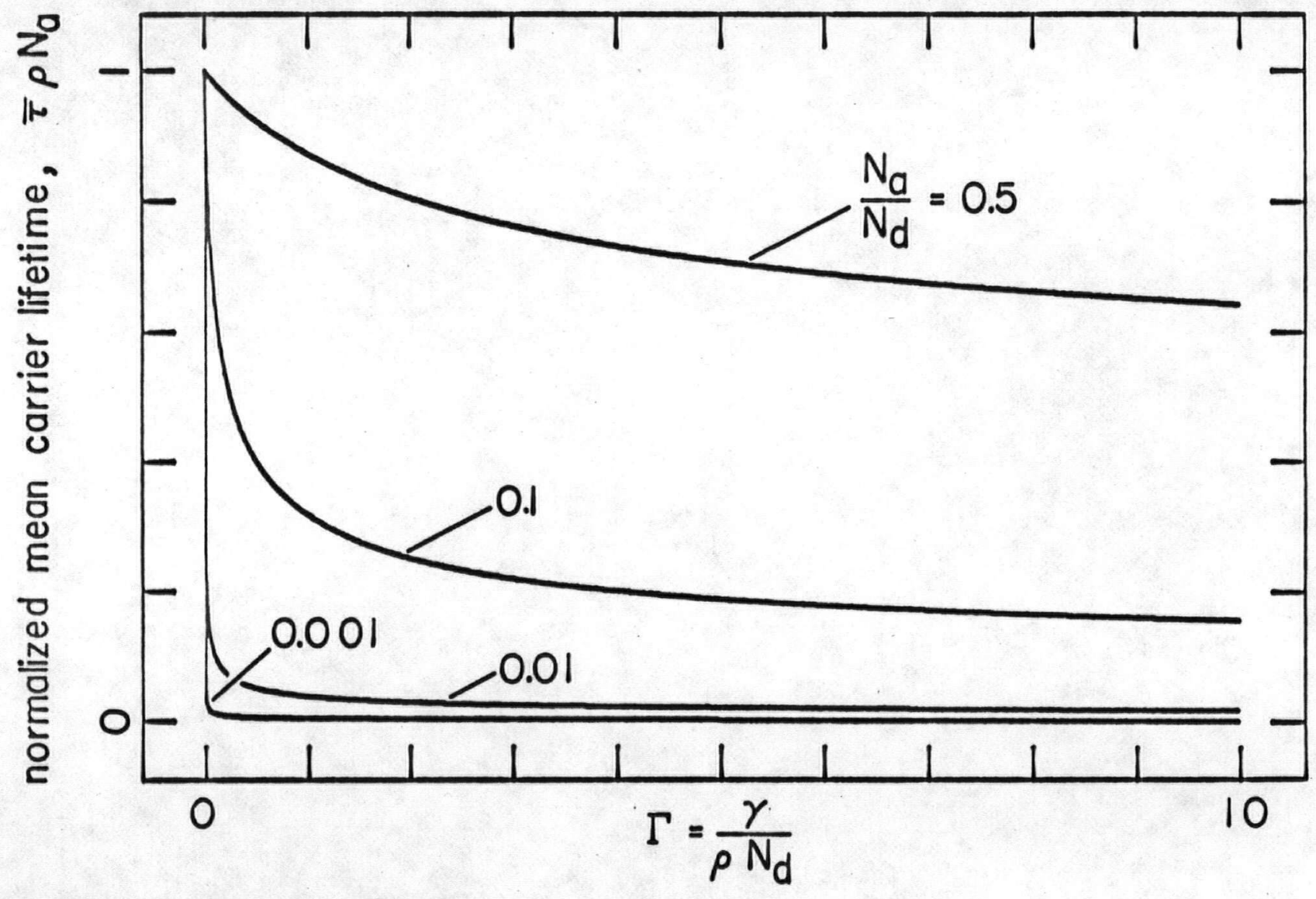

generation rate

Fig.(2.18) - Normalized mean carrier lifetime versus generation rate. 


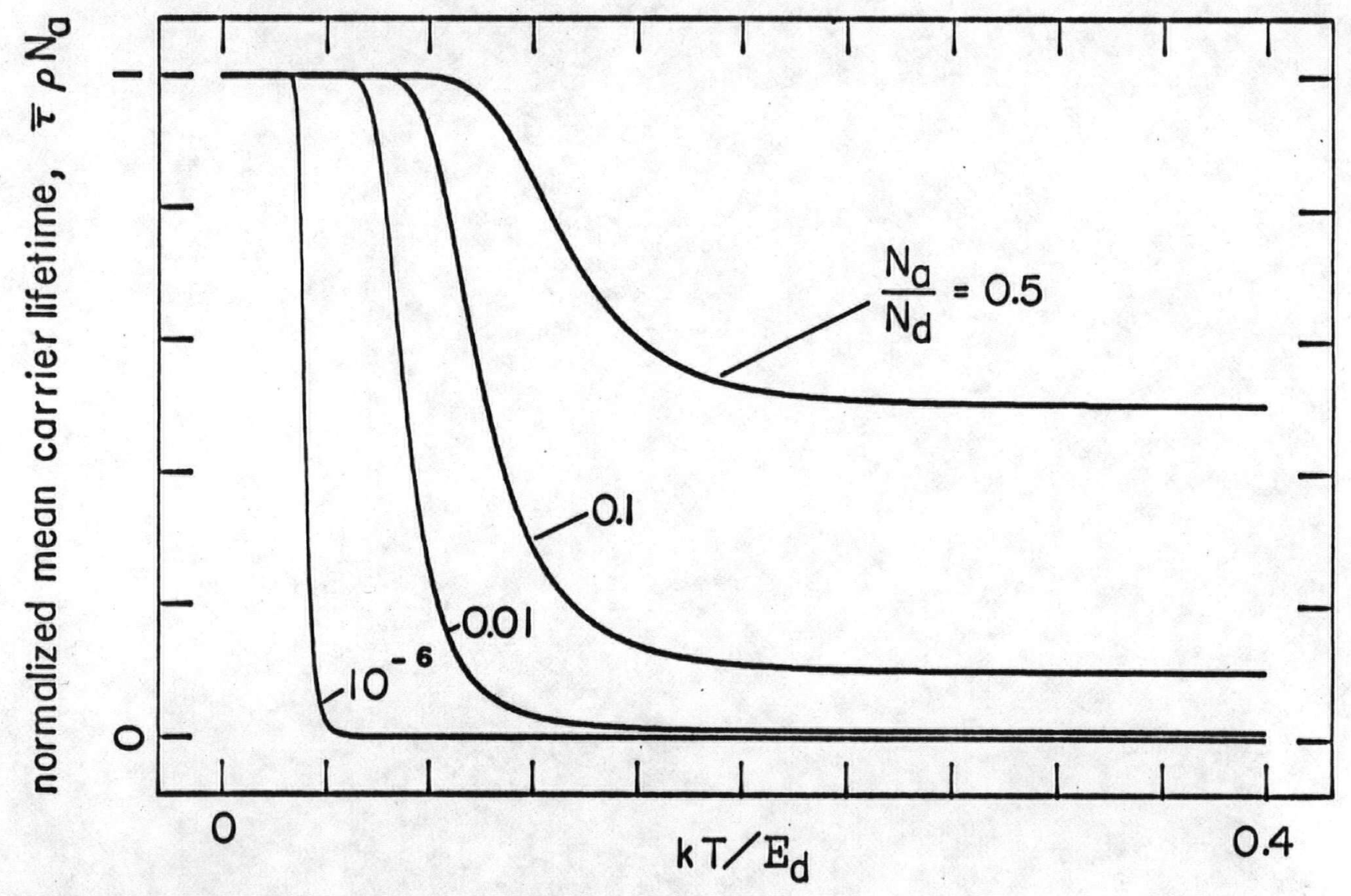

dimensionless temperature

Fig.(2.19) - Normalized mean carrier lifetime versus dimensionless temperature. 
$\delta \gamma \ll \gamma$,

$\gamma \bar{N} \ll \bar{N}$, and

$\delta \gamma \delta \bar{N} \ll \delta \bar{N}$

are made, then

$$
\begin{aligned}
& \delta \gamma\left(N_{d}-N_{a}-\bar{N}\right)+\gamma\left(N_{d}-N_{a}-\bar{N}\right)=\rho\left(N_{a}+\bar{N}\right) \\
& +\rho \delta \bar{N}\left(2 \bar{N}+N_{a}+\gamma / \rho\right) .
\end{aligned}
$$

Substitution of equation (2.45) into equation (2.48) leads to the expression

$$
\delta \gamma\left(N_{d}-N_{a}-\bar{N}\right)=\delta \bar{N}\left[\rho\left(2 \bar{N}+N_{a}\right)+\gamma\right]
$$

Finally, the responsivity $R$, is given by

$$
R=\frac{\delta \bar{N}}{\delta \gamma}=\frac{N_{d}-N_{a}-\bar{N}}{\rho\left(2 \bar{N}+N_{a}\right)+\gamma},
$$

which is expressed in terms of the fractional ionization and the compensation ratio as

$$
R=\frac{1}{\rho} \frac{(1-f)^{2}(1-\lambda)}{f(2-f)(1-\lambda)+\lambda}
$$

Figures (2.20), (2.21), and (2.22) illustrate the responsivity as functions of $f, \Gamma$, and $k T / \varepsilon_{d}$ respectively. Once again, the normalization was necessary to accommodate the different compensation levels on one plot. 


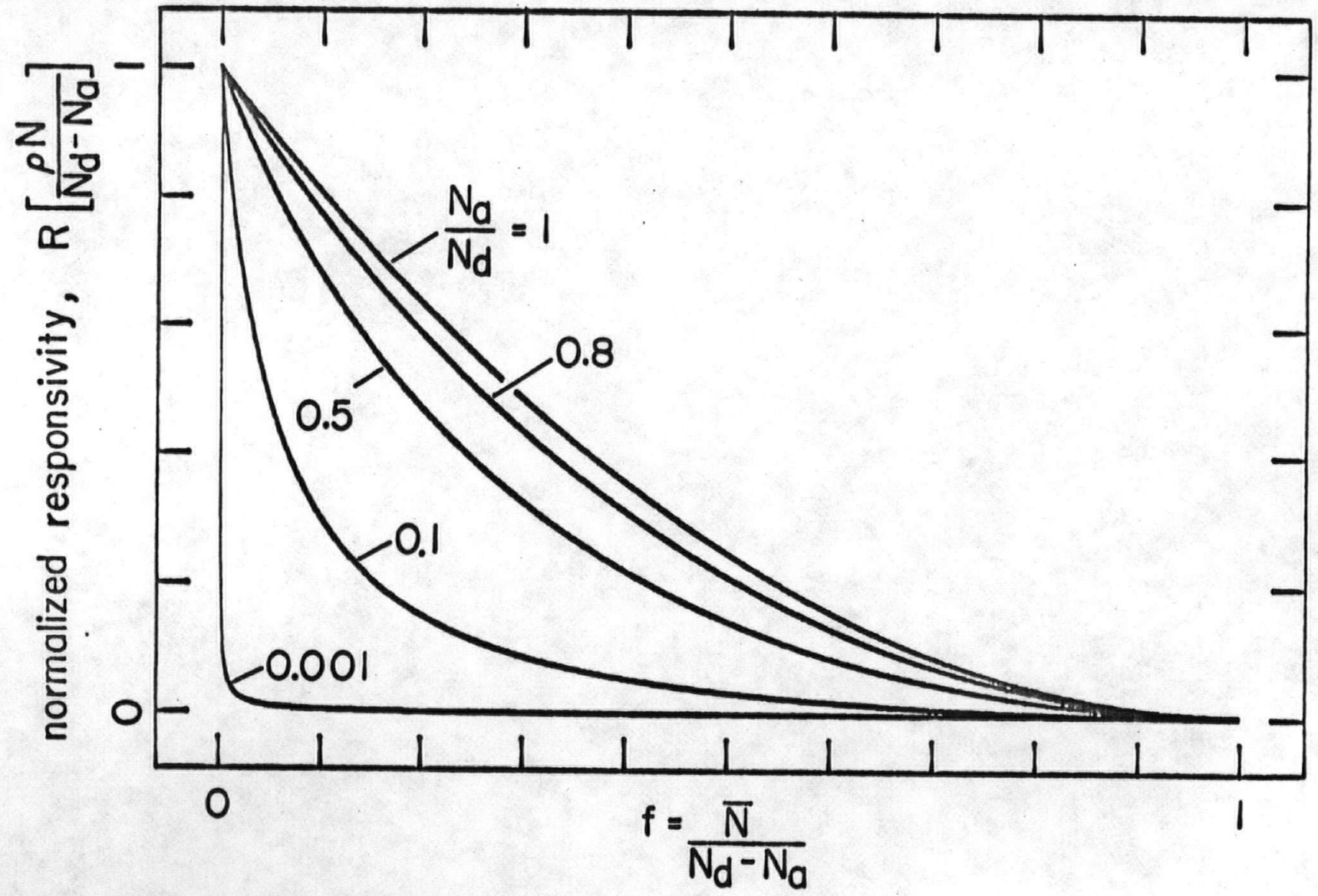

fractional ionization

Fig.(2.20) - Normalized responsivity versus fractiunal ionization. 


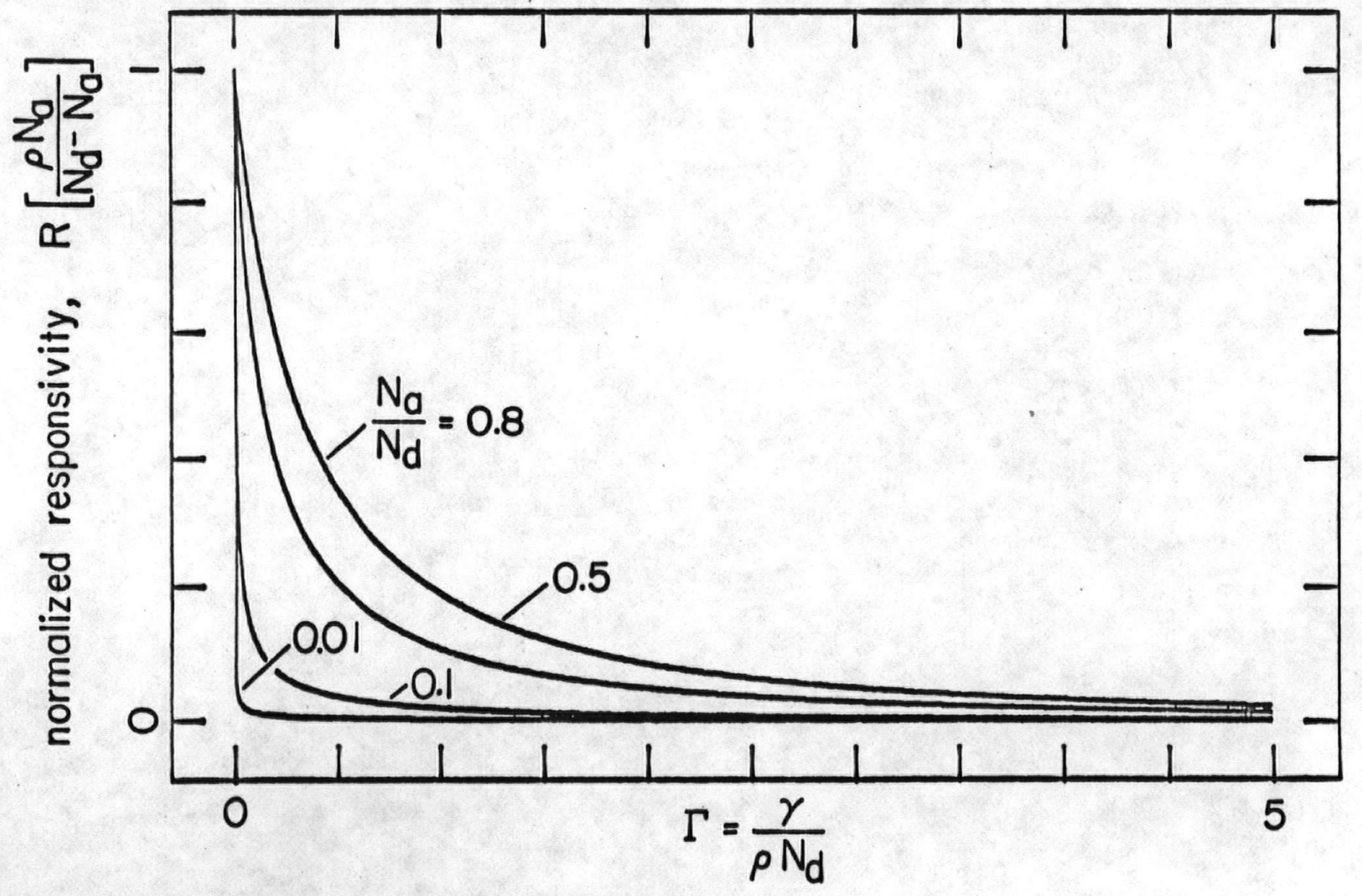

generation rate

Fig.(2.21) - Normalized responsivity versus generation rate. 


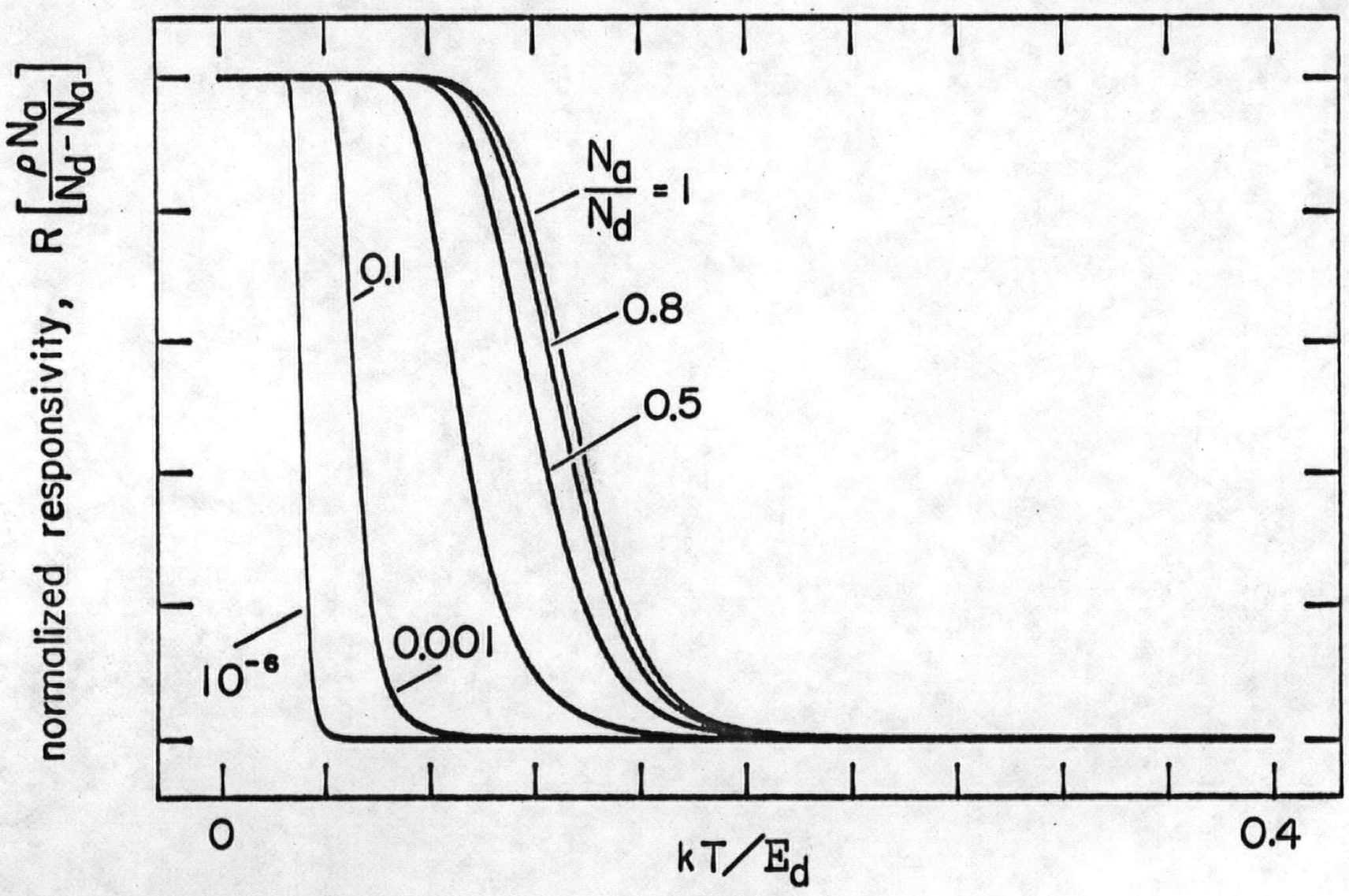

dimensionless temperature

Fig.(2.22) - Normalized responsivity versus dimensionless temperature. 
The noise equivalent power, or NEP, of a given detector is defined as the value of the signal power for which the signal-tonoise ratio is equal to unity. It is derived as follows: The generation coefficient of equation (2.1) can be expressed as

$$
\gamma=\gamma_{t h}+\frac{\eta}{h v} \frac{\left(P_{B}+P_{S}\right)}{\left(N_{d}-N_{a}\right)}=\gamma_{t h}+\gamma_{B}+\gamma_{s},
$$

where $\gamma_{t h}$ is the thermal contribution. $P_{B}$ and $P_{S}$ are the background and signal powers respectively. $\gamma_{B}$ and $\gamma_{S}$ represent the background and signal contributions to the generation coefficient respectively. $\eta$ is defined as the quantum efficiency, $h$ is Planck's constant, and $v$ is the optical frequency.

An incremental change in the signal power $P_{S}$ will lead to a change in the generation rate $\gamma$ of $\delta \gamma_{S}$ and perturb $\bar{N}$ by an amount $\delta \bar{N}$, which gives

$$
\left(\gamma+\delta \gamma_{S}\right)\left(N_{d}-N_{a}-\bar{N}-\delta \bar{N}\right)=\rho(\bar{N}+\delta \bar{N})\left(N_{a}+\bar{N}+\delta \bar{N}\right) .
$$

Combining terms, using the assumptions of equation (2.47), and solving for the change in $\overline{\mathrm{N}}$ of $\delta \overline{\mathrm{N}}$ results in

$$
\delta \bar{N}=\frac{\delta \gamma_{S}\left(N_{d}-N_{a}-\bar{N}\right)}{\rho\left(N_{a}+2 \bar{N}\right)+\lambda}
$$

Solving for $\gamma / \rho$ when equation (2.1) is set equal to equation (2.2) yields 


$$
\frac{\gamma}{\rho}=N_{d} \frac{f[\lambda+f(1-\lambda)]}{(1-f)}
$$

and upon substituting into equation (2.54) results in

$$
\delta \bar{N}=\frac{\delta \gamma_{S}}{\rho} \frac{(1-\lambda)(1-f)^{2}}{f(2-f)(1-\lambda)+\lambda}
$$

The signal-to-noise ratio relates to the signal current and noise current by ${ }^{12}$

$$
\left(\frac{S}{N}\right)^{2}=\frac{\bar{i}_{s}^{2}}{\bar{i}_{g r}^{2}} .
$$

The mean signal current is given by equation (2.20) as

$$
\bar{i}=\frac{e V}{L^{2}} \mu_{n} \delta \bar{N} .
$$

The mean-square $g-r$ current noise of equation (2.31) can be expressed in terms of $f$ and $\lambda$ as

$$
\bar{i}_{g r}^{2}=\frac{4}{\rho}\left(\frac{e V \mu_{n}}{L^{2}}\right)^{2} \frac{f(1-\lambda)(1-f)^{2}[\lambda+f(1-\lambda)] \Delta f}{[f(2-f)(1-\lambda)+\lambda]^{2}} .
$$

Substitution into equation (2.57) yields

$$
\left(\frac{S}{N}\right)^{2}=\frac{(\delta \bar{N})^{2}}{4} \rho \frac{[f(2-f)(1-\lambda)+\lambda]^{2}}{f(1-\lambda)(1-f)^{2}[\lambda+f(1-\lambda)] \Delta f} .
$$

Defining $\delta \gamma_{S}$ in equation (2.56) to have the form

$$
\delta \gamma_{S}=\frac{n P_{S}}{h v\left(N_{d}-N_{a}\right)}
$$


and substituting for $(\delta \bar{N})^{2}$ in equation (2.60) results in

$$
\left(\frac{S}{N}\right)^{2}=\frac{1}{4 \rho \Delta f}\left(\frac{\eta}{h v}\right)^{2} \frac{P_{S}^{2}}{\left(N_{d}-N_{a}\right)^{2}} \frac{(1-\lambda)^{2}(1-f)^{2}}{f(1-\lambda)[\lambda+f(1-\lambda)]} .
$$

The NEP, given by setting equation (2.62) equal to unity, is

$$
N E P=\frac{h v}{\eta} \sqrt{4 p \Delta f}\left(N_{d}-N_{a}\right)\left[\frac{f(1-\lambda)[\lambda+f(1-\lambda)]}{(1-\lambda)(1-f)}\right]^{\frac{1}{2}} .
$$

The NEP is plotted as functions of $f, \Gamma$, and $k T / \varepsilon_{d}$ in figures (2.23), (2.24), and (2.25) respectively.

One will note that in figure $(2.24)$ the lower most curve represents the NEP of an ideal photon detector. The NEP of the extrinsic photoconductive detector is known to be greater than that of the ideal photon detector by at least a factor of the square root of two. This results from the fact that the fluctuations in the generation and recombination rates are comprised of two statistically independent noise sources compared to the ideal photon detector which is limited only by photon noise. The NEP of the ideal photon detector is given by ${ }^{13}$

$$
\mathrm{NEP}_{\text {Ideal }}=\left[\frac{4 \mathrm{P}_{B} h \nu \Delta f}{\eta}\right]^{\frac{1}{2}}
$$

In the theoretical calculation of the NEP above, the generation coefficient due to the background was given by

$$
\gamma_{B}=\frac{\eta P_{B}}{h v\left(N_{d}-N_{a}\right)}
$$




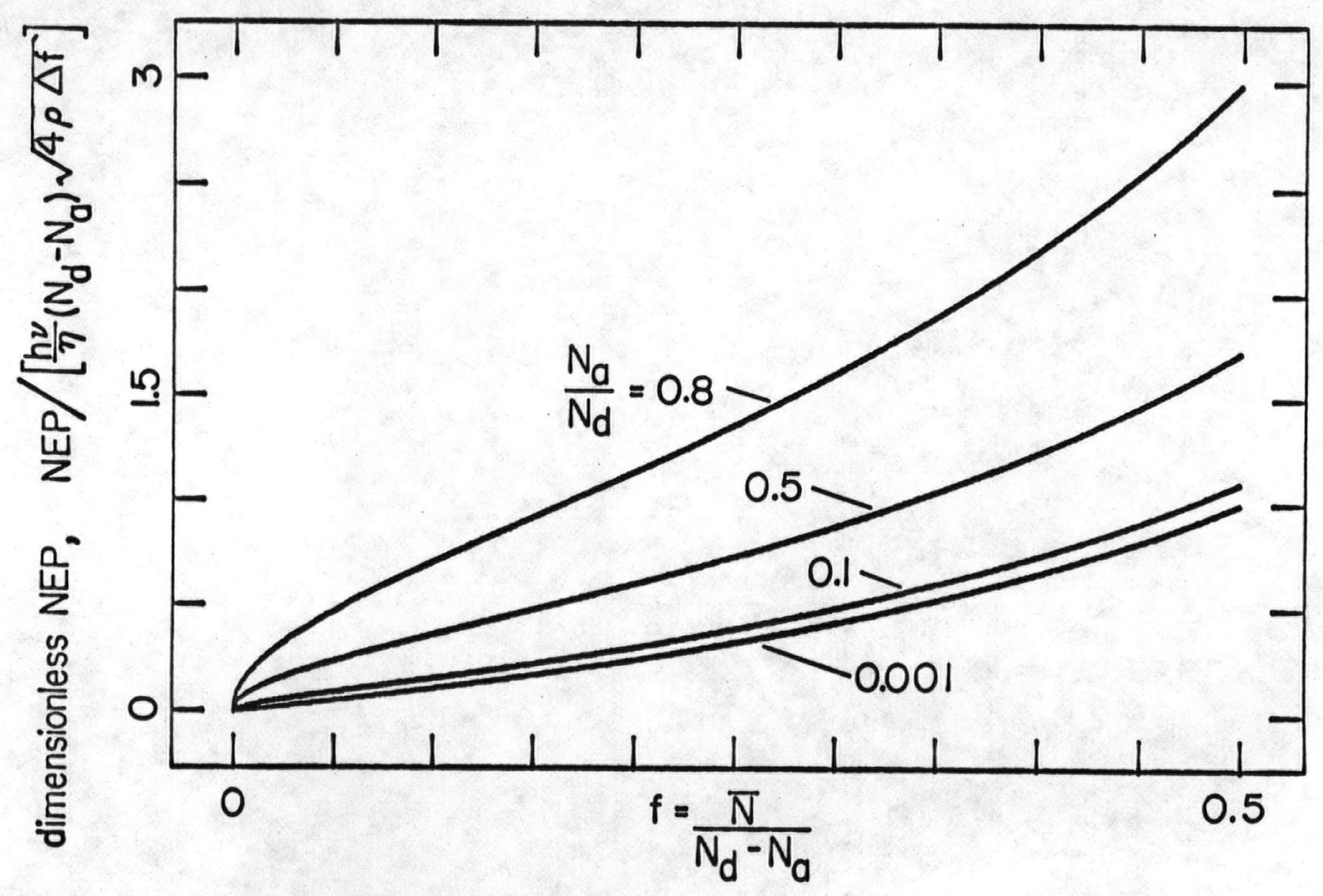

fractional ionization

Fig.(2.23) - Dimensionless NEP versus fractional ionization. 


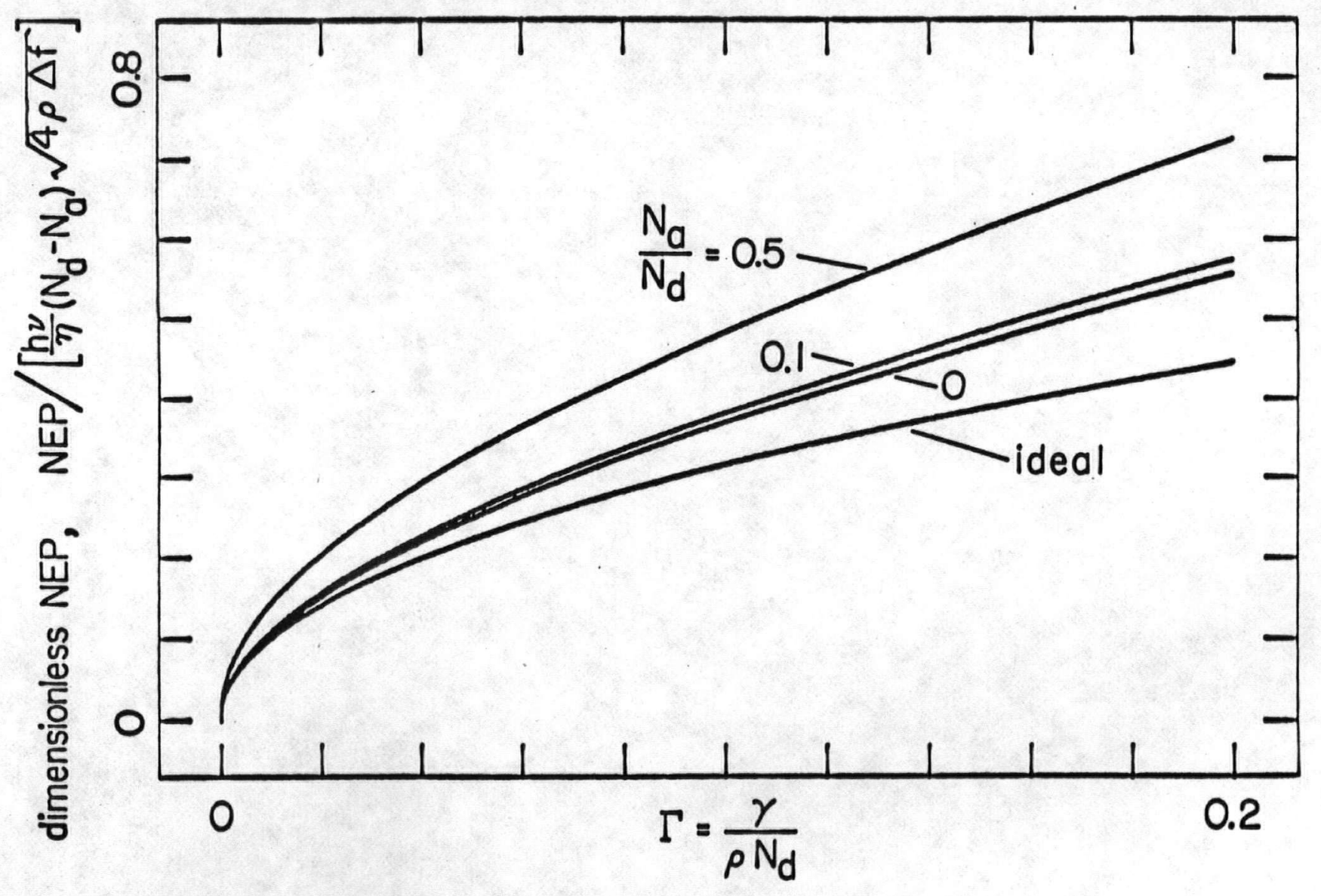

generation rate

Fig.(2.24) - Dimensionless NEP versus generation rate. 


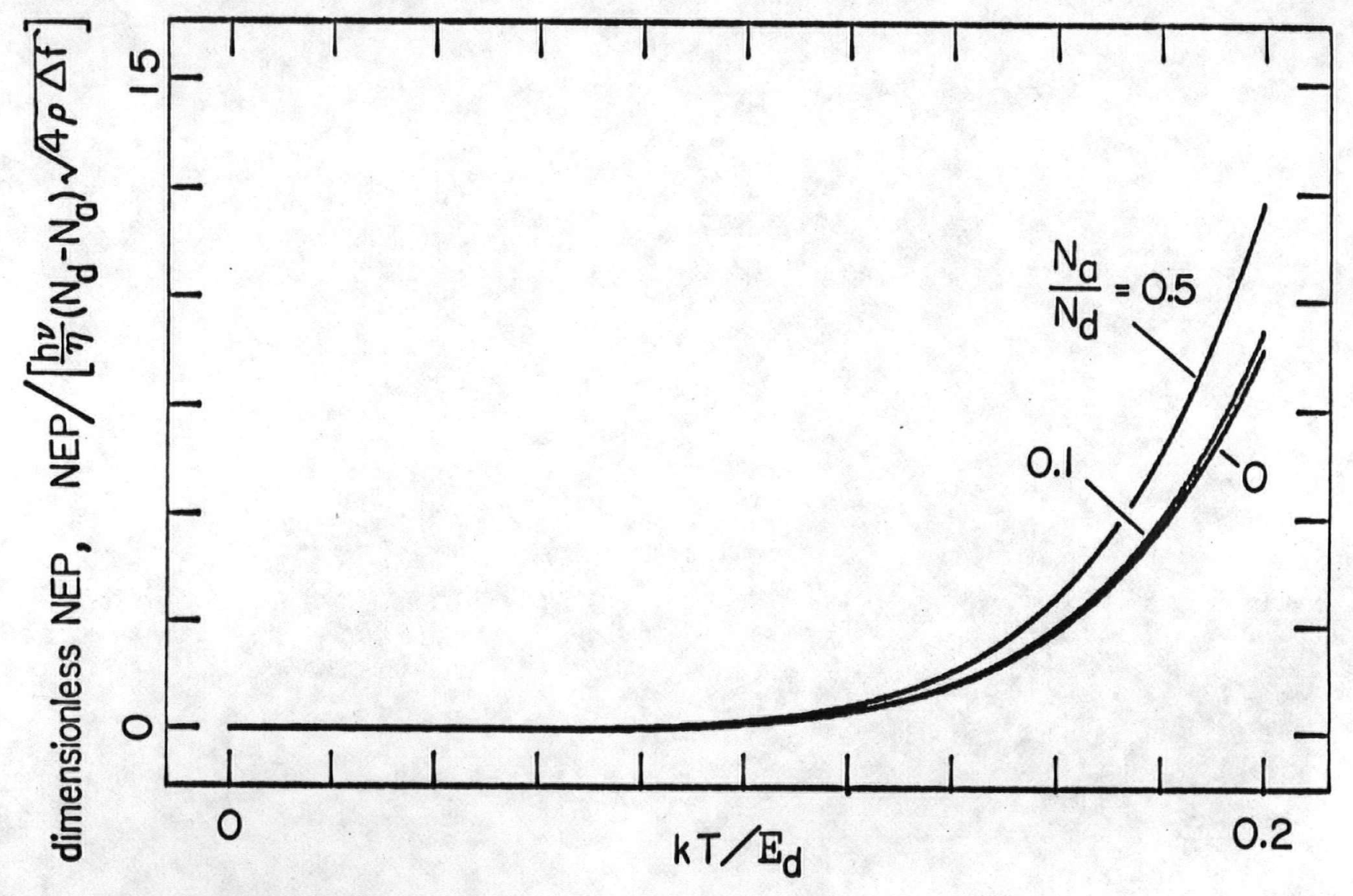

dimensionless temperature

Fig.(2.25) - Dimensionless NEP versus dimensionless temperature. 
Substituting equations (2.55) and (2.65) into equation (2.64) results in an ideal NEP given by

$\mathrm{NEP}_{\text {Ideal }}=\frac{h v}{\eta} \sqrt{4 \rho \Delta f} \sqrt{N_{d}\left(N_{d}-N_{a}\right)} \quad\left[\frac{f(1-\lambda)[\lambda+f(1-\lambda)]}{(1-\lambda)(1-f)}\right]^{\frac{1}{2}}$.

The ideal NEP can be expressed equivalently as

$$
\mathrm{NEP}_{\text {Ideal }}=\left[\frac{h v}{\eta} \sqrt{4 \rho \Delta f} \mathrm{~N}_{\mathrm{d}}\right] \sqrt{\Gamma} .
$$

For the ideal detector $\mathrm{N}_{\mathrm{a}}$ is zero. Thus, the ratio of the theoretical NEP for the impurity compensated extrinsic photoconductive detector to the NEP of the ideal photon detector is

$$
\frac{\text { NEP }_{g-r} \text { theory }}{{ }^{N E P} \text { Ideal }}=\frac{1}{(1-\lambda)(1-f)} .
$$

\subsection{Conclusion}

This chapter presented a complete theory of the generationrecombination noise associated with extrinsic photoconductive detectors. The theory accounts for the effect of impurity compensation on detector properties and stresses that this effect is often far from negligible.

The treatment of NEP allows for the calculation of the magnitude of the recombination coefficient $\rho$, from an empirical fit of one's data to the plots provided. Insight as to the functional form of this coefficient may be gained from such analyses. 
It is hoped that this theory will provide a means for improved characterization of this class of detectors. We also hope that this theory will lead to improved photoconductive materials by providing the detector manufacturer with insight to better design criteria. 


\section{REFERENCES}

1. Reference 2, Chapter 1.

2. Reference 1, Chapter 1.

3. R.E. Burgess, The Statistics of Charge Carrier Fluctuations in Semiconductors, (Proceedings of the Physical Society of London, B 69, 1955).

4. Reference 2, Chapter 1.

5. Reference 3, Chapter 2.

6. R.W. Boyd, Radiometry and the Detection of Optical Radiation, (Wiley-Interscience, New York, 1983) pp. 169-176.

7. Reference 6, Chapter 2, page 172.

8. Reference 3, Chapter 2.

9. Reference 6, Chapter 2, page 170.

10. Reference 6, Chapter 2, page 176.

11. R.A. Smith, The Detection and Measurement of Infrared Radiation, (Oxford University, London, 1968). 
CHAPTER 3

EXPERIMENTAL SETUP OF A Ge:Ga DETECTION SYSTEM

\subsection{Introduction}

The aim of this chapter is to present a detailed description of the constituent components of the 100 micron detection system used in the experimental phase of the author's research. Figure (3.1) illustrates the experimental setup of the Ge:Ga detection system.

The system was used to take responsivity and noise data, first as a function of the detector temperature and secondly as a function of photon flux from a blackbody source. In chapter 4, a comparison is made between the experimental and theoretical results and the initial tests leading to system optimization are reviewed.

\subsection{The Ge:Ga Detector}

The Ge:Ga extrinsic photoconductive detectors used in this research were given to the author by Don Langford of Jet Propulsion 


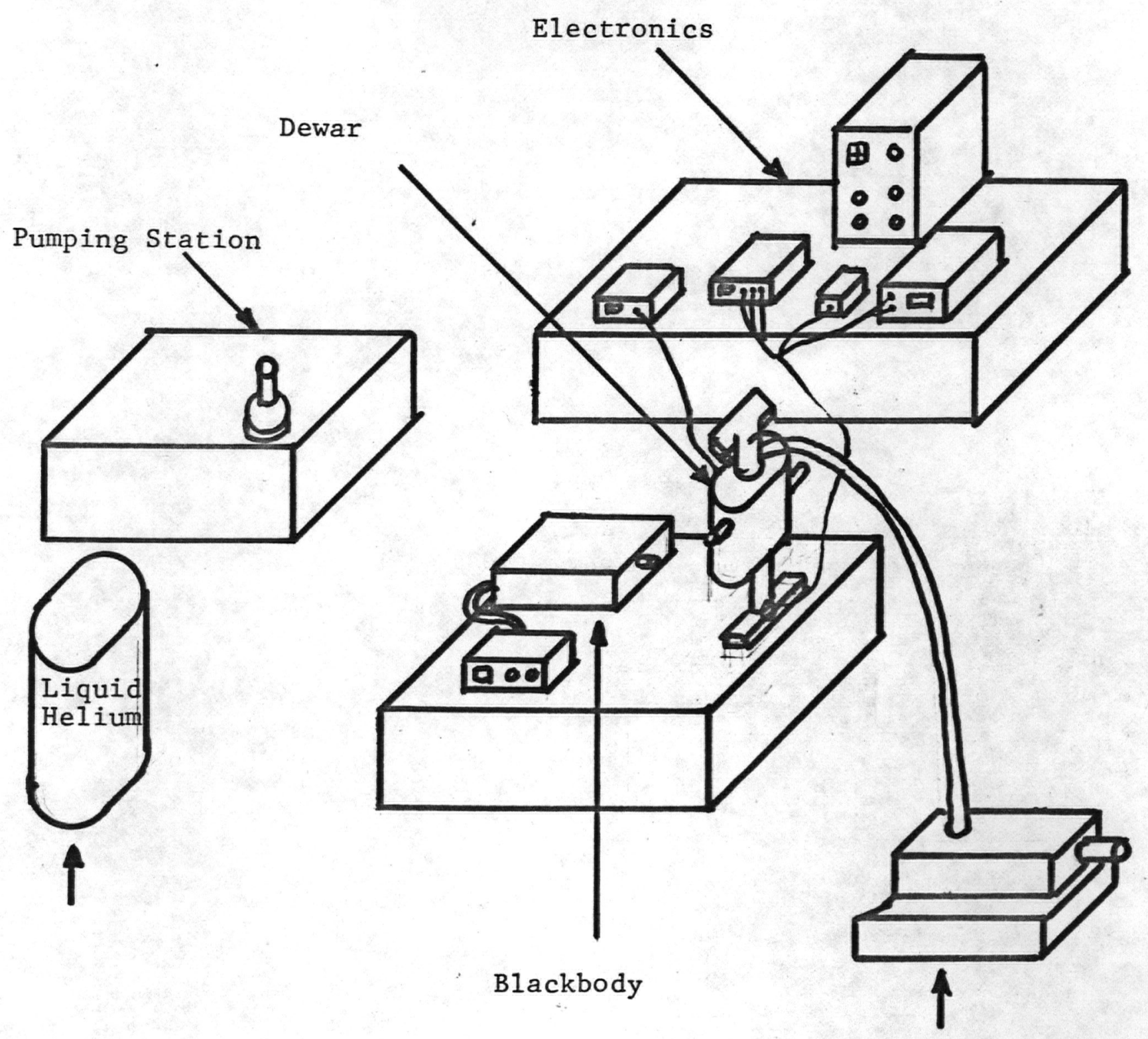

Helium Resevoir Pump

Fig.(3.1) - Experimental Setup 
Laboratory. They are the type that were used in the Infrared Astronomy Satellite (IRAS) focal plane. The peak response of these detectors occurs at a wavelength of 101 microns as shown by the response curve of figure (3.2). ${ }^{1}$

The energy band diagram shown in figure (3.3) illustrates the impurity levels in the Ge:Ga detectors. The germanium was doped with gallium to a concentration of approximately $2.5 \times 10^{14} \mathrm{~cm}^{-3}$. The compensating or residual donor concentration before doping was not known, but was assumed to be less than $5 \times 10^{11} \mathrm{~cm}^{-3} \cdot 2$ The detector measures only $1.5 \times 1.5 \times 1.4 \mathrm{~mm}^{3}$, which made soldering it to its pedestal a difficult task. The electrical contacts were made by boron ion implant and metallization by evaporation of chromium and then gold.

The detector mount and integrating cavity are illustrated in figure (3.4). One electrode of the detector is soldered to a pedestal on the mount using low temperature indium solder. A hair-thin gold wire is soldered to the remaining electrode and is connected to the signal lead. A $1 \mathrm{~mm}$ diameter hole was drilled into the side of the integrating cavity which fits over the detector. The purpose of the integrating cavity is to increase the responsivity of the detector by reflecting light for multiple passes through the detector. This results in a quantum efficiency approaching unity. The detector is oriented at $45^{\circ}$ with respect to the line of sight to eliminate fresnel reflections and to reduce uneven illumination. ${ }^{3}$ 
52

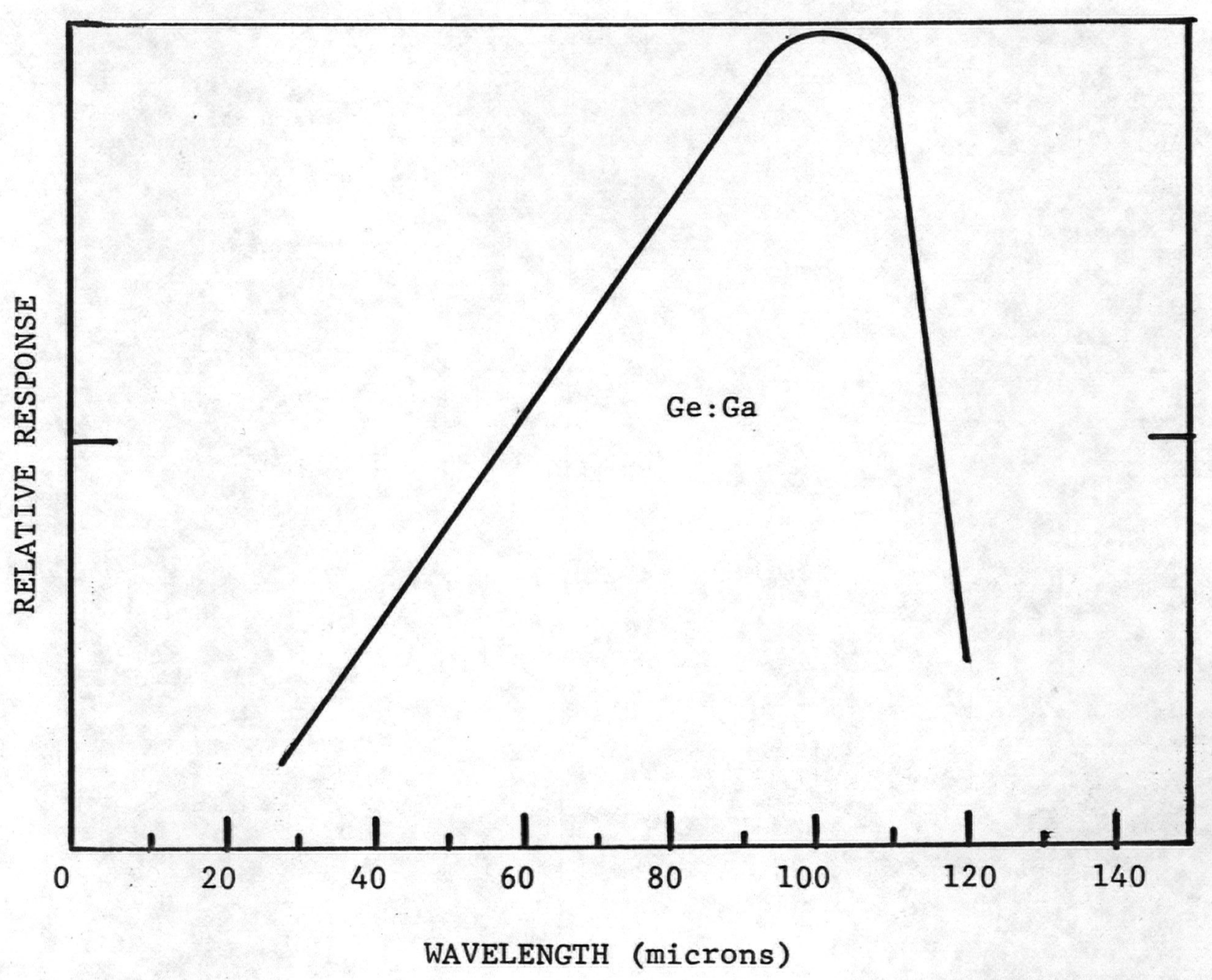

Fig(3.2) - Relative Wavelength Response of Ge:Ga Detector 


\section{IMPURITY LEVELS IN GE:GA AT $2.5^{\circ} \mathrm{K}$}
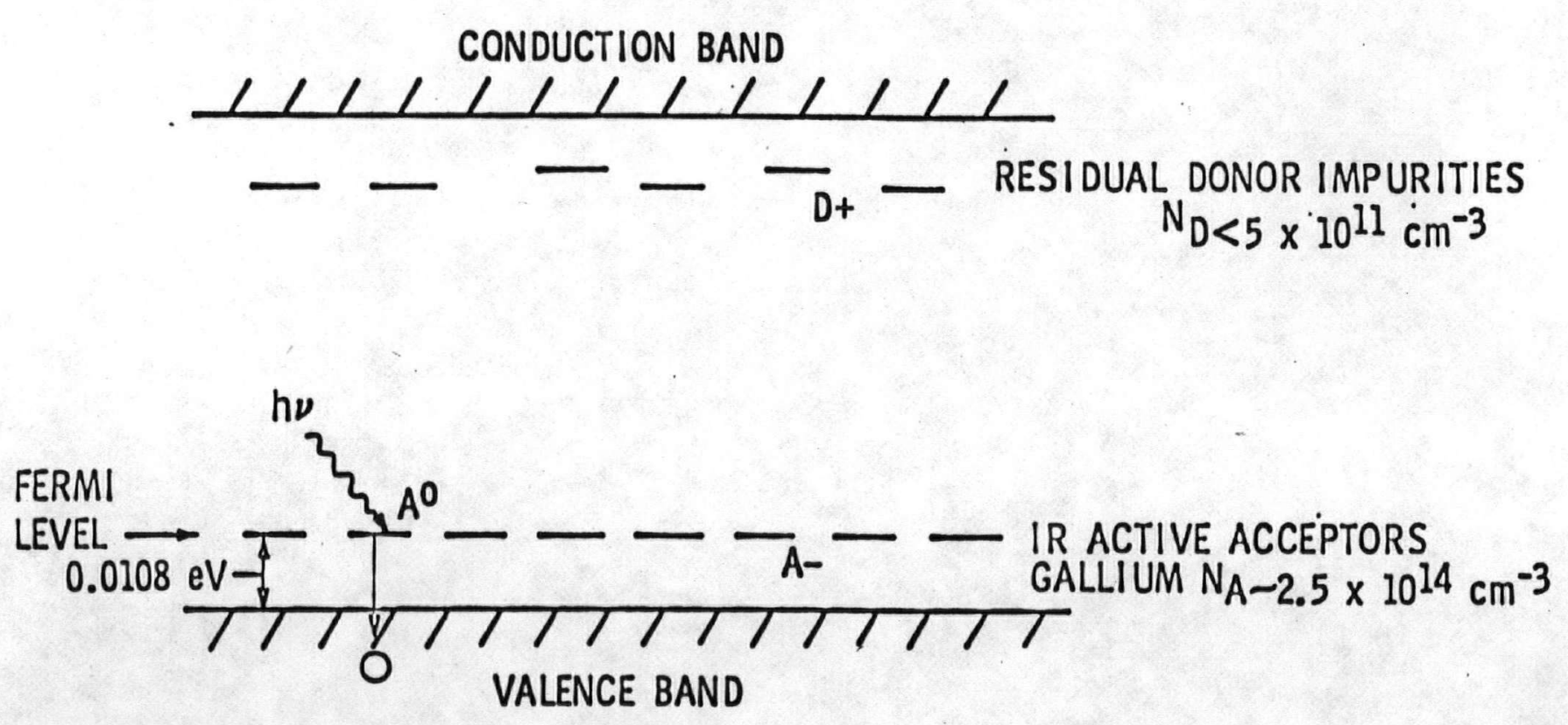

Fig.(3.3) - Impurity levels in Ge:Ga at 2.5 degrees Kelvin. 
Mounting Hole for

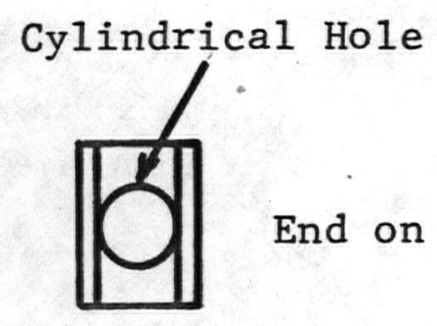

Power Resistor

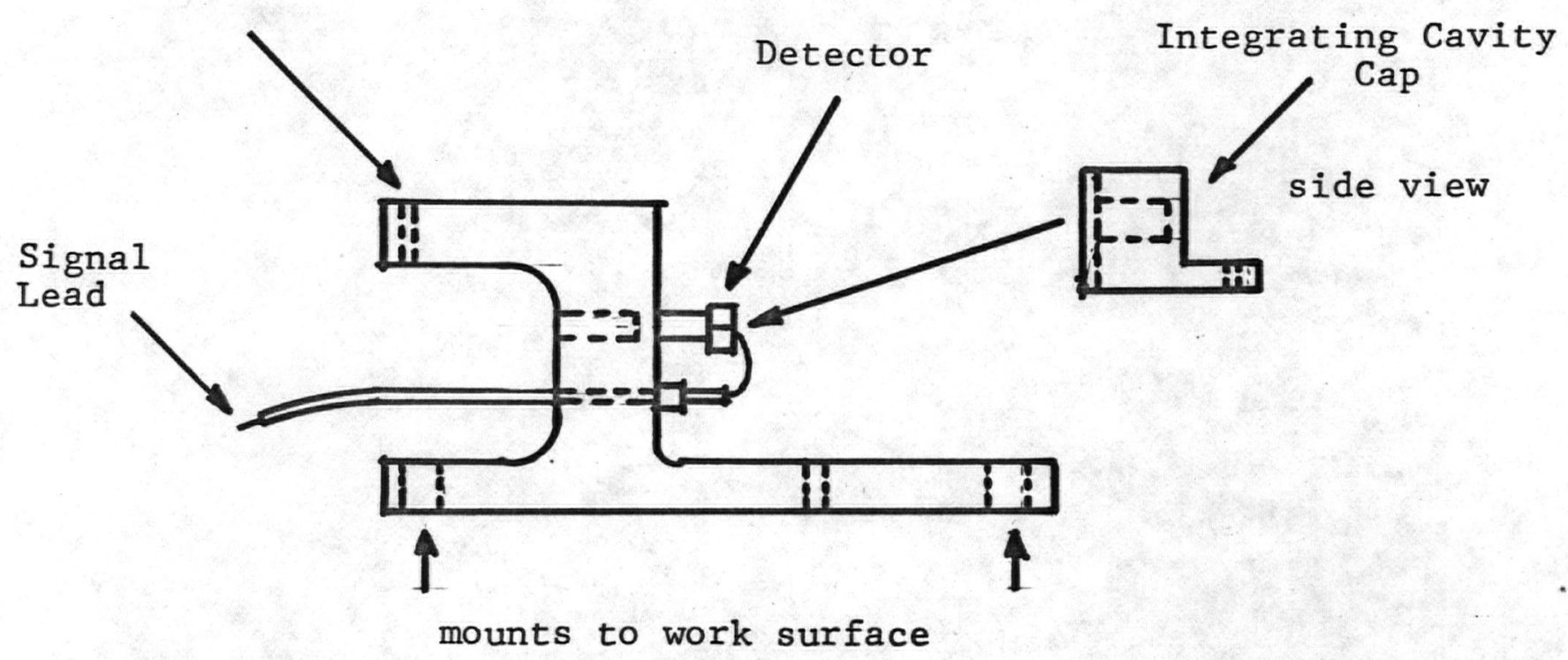

Fig(3.4) - DETECTOR MOUNT AND INTEGRATING CAVITY 


\subsection{Helium Dewar}

The dewar shown in figure (3.5) is a modified version of a model HLDT-1 Helium Dewar made by Hofman Laboratories Inc., circa 1965. The primary alteration involved extending the work surface by approximately 7 inches to the level of the windows. This configuration solved the problems of two previously considered designs. The first design considered was to leave the work surface where it was, several inches from the window, and employ cooled reflective optics to project radiation down the inside of the cold shield. This presented two problems, however; alignment and a restricted field of view. The second design that was given some consideration involved the mounting of a 7 inch copper rod to the old work surface (at the base of the cold shield), forming a new work surface at the window level. This eliminated the need for reflective optics, but did pose another problem; the thermal resistance was quite appreciable and temperatures below $17^{\circ}$ Kelvin could not be reached. Extension of the helium reservoir to the window level solved both of these design problems, resulting in a direct line of sight to the detector through the window and permitting temperatures approaching $4.2^{\circ}$ Kelvin to be reached (temperatures as low as $2^{\circ}$ Kelvin could be reached by pumping on the helium reservoir).

A gold-plated cold shield maintained at a temperature approaching $77^{\circ}$ Kelvin by liquid nitrogen surrounds the extended helium reservoir tube and work surface on which the detector holder is mounted as illustrated in figure (3.6). The cold shield also serves 


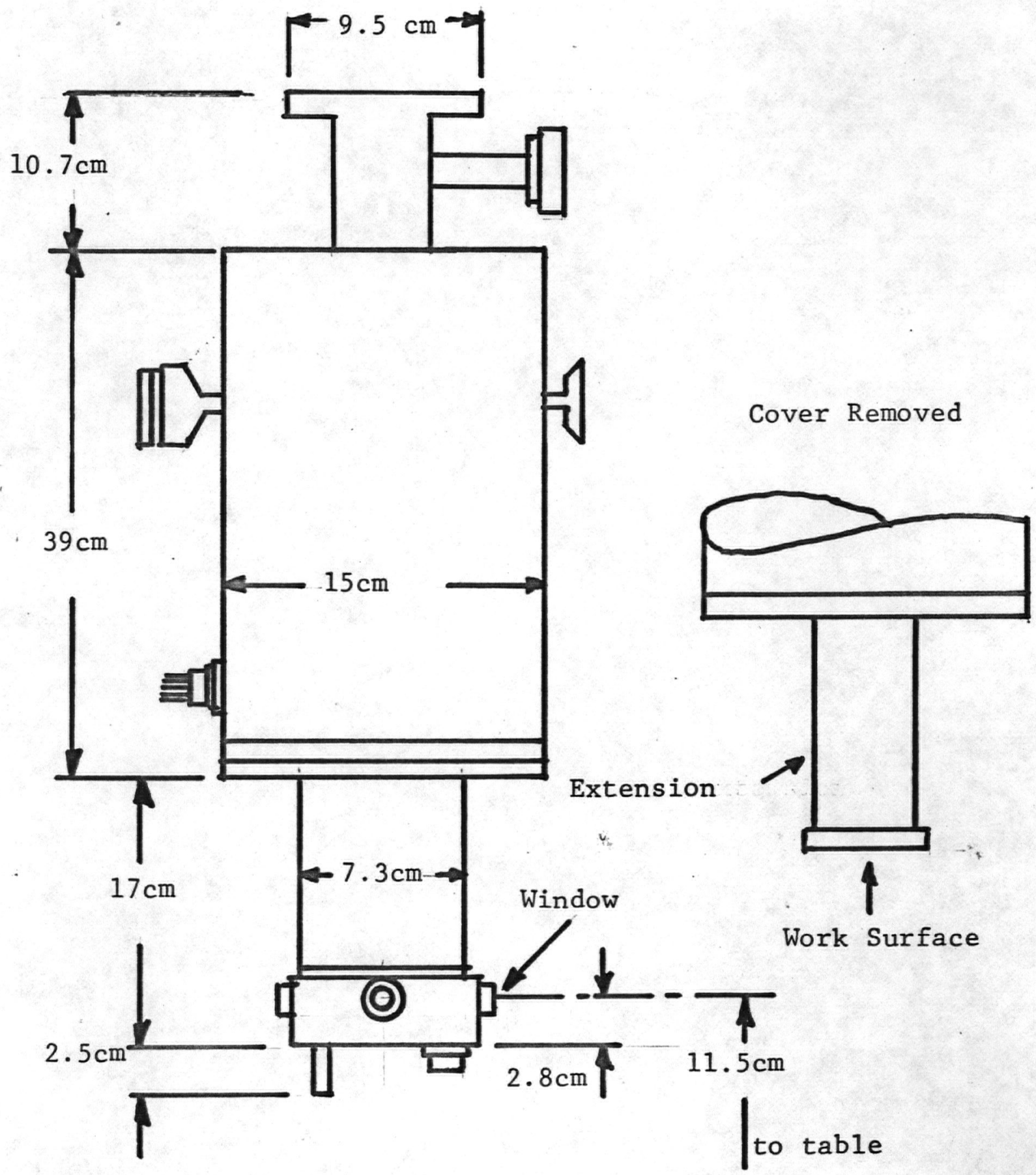

Fig. (3.5) - HELIUM DEWAR 


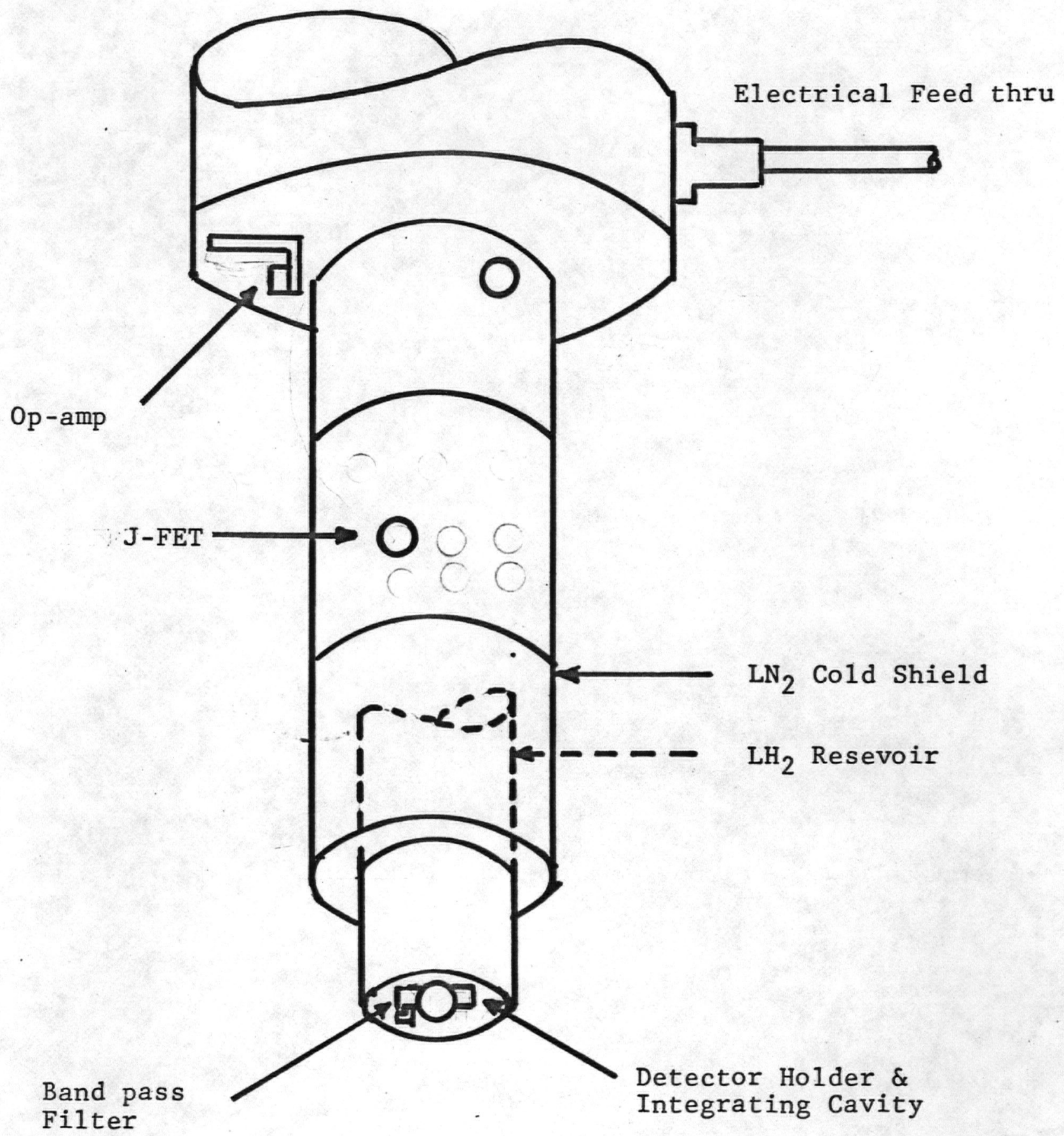

Fig. (3.6) - Cryostat cold shield \& work surface. 
as a mount for the detector's amplifier (with the exception of the operational amplifier which is maintained at room temperature).

A typical two-stage pumping station was used to evacuate the dewar, consisting of a mechanical roughing pump and a diffusion pump with a cold trap. Pressure was monitored by an ionization vacuum gauge of type GIC-110A made by Consolidated Vacuum Corporation. More than twenty hours were required to reach a pressure of $7 \times 10^{-6}$ Torr. This pressure was considered adequate for the experiment, however, because of the inevitable cryopumping due to the liquid helium. This long recycling time proved to be a major obstacle in rendering the detection system operational.

Two electrical feed-throughs were used to accommodate the detector electronics. A standard two-pin feed-through on the bottom of the cover was connected to the power resistor. The eight-pin feed-through on the side of the dewar was constructed by the author to accommodate the remaining electronics.

The inside of the cover of the dewar was painted with black primer paint in order to reduce stray radiation. The inside and outside of the cold shield were also painted black. The black paint reduces the background photon flux and allows for a more accurate calibration of the detector.

\subsection{Detector Electronics}

Figure (3.7) illustrates in block-diagram form the electronic components needed to operate and analyze the experiment. The primary 


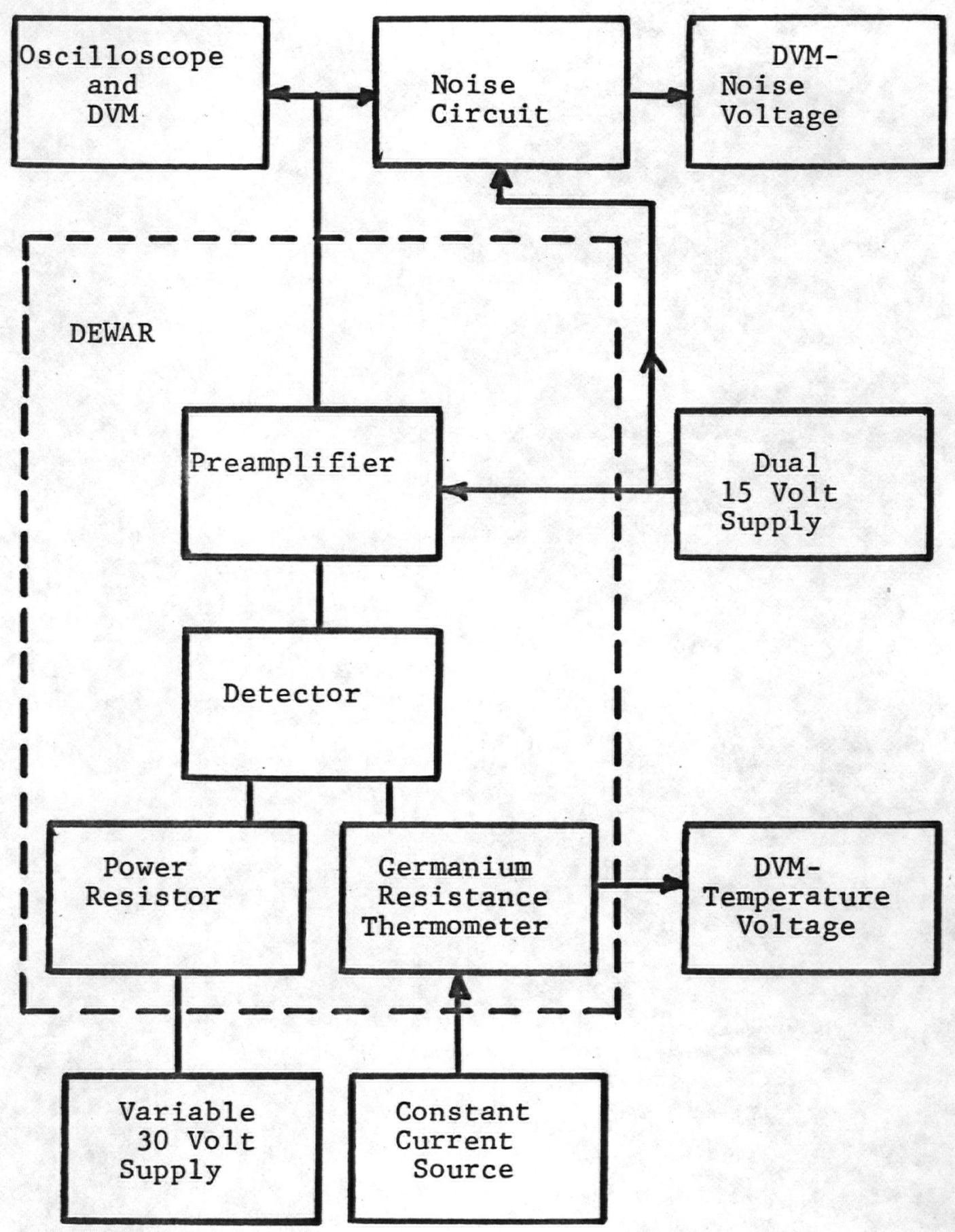

Fig. (3.7) - BLOCK DIAGRAM OF ELECTRONICS 
components include a low noise preamplifier to measure the detector responsivity, a noise circuit to measure the rms noise voltage, and a germanium resistance thermometer. The preamplifier and noise circuits were powered by a dual 15 volt D.C. power supply made in our laboratory. A variable 30 volt power supply regulated the $1 \mathrm{k} \Omega$ power resistor. The constant current source for the thermometer was powered by a 9 volt transistor battery.

The final design of the low-noise preamplifier is illustrated in figure (3.8). It functions as a noninverting amplifier with a voltage gain $G$, of

$$
G=\frac{V_{\text {OUT }}}{V_{B}}=1+\frac{R_{f}}{R_{D}},
$$

where $V_{\text {OUT }}$ is the output of the preamplifier, $V_{B}$ is the bias voltage of the detector, $R_{f}$ is the feedback resistance, and $R_{D}$ is the detector resistance. Don Langford of Jet Propulsion Laboratory supplied the design from which the final design evolved. Consideration had to be given as to how the detector should be biased. The circuit was designed as to allow one of the detector electrodes to be soldered directly to the pedestal, providing both a ground to complete the bias circuit and excellent thermal contact.

The LM108 operational amplifier manufactured by National Semiconductor was selected for its characteristics of very low noise and a high input impedence of about $10^{14}$ ohms. Such a high input impedence is necessary when coupling the device to detector and feedback resistances on the order of $10^{10}$ ohms. 


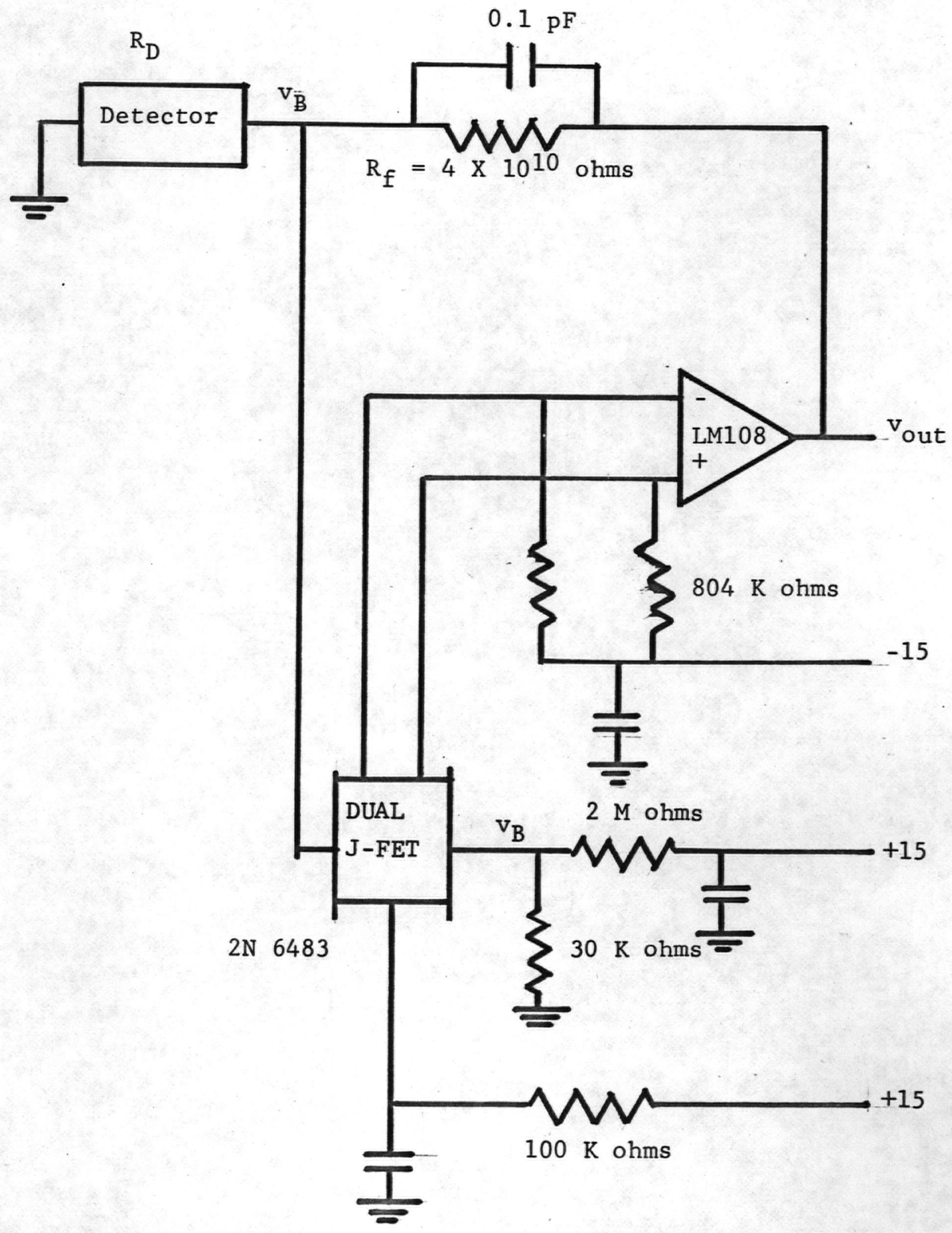

Fig. (3.8) - PREAMPLIFIER CIRCUIT 
The 2N6483 dual-matched J-FET was also chosen for its low noise characteristics. It functions to minimize unwanted fluctuations between the inputs to the operational amplifier. An $804 \mathrm{k} \Omega$ resistor was placed between each input to the operational amplifier and the -15 volt connection to provide a current path to drive the J-FET.

To prevent the amplifier from being driven into oscillation, a 0.1 pf capacitor was placed in parallel with the $4 \times 10^{9}$ ohm feedback resistor. This resulted in an RC-time constant that limited the spectral content of fluctuations to less than $2.5 \mathrm{kHz}$.

Cooling of the circuit elements further improves noise characteristics of the output signal by reducing the thermal Johnson noise associated with all resistive elements. Because of the extremely high resistance of the feedback resistor, it was maintained at the same low temperature as that of the detector, ranging between $2^{\circ}$ and $5^{\circ}$ Kelvin. The remainder of the circuit, with the exception of the operational amplifier, was attached with teflon tape to a copper tube that fit over the outside of the cold shield. The components were maintained at a temperature approaching $77^{\circ}$ Kelvin. The operational amplifier was maintained at room temperature because it would not function at $77^{\circ}$ Kelvin. Consideration had to be given to the temperature coefficient of the biasing resistors, as cooling them resulted in a 0.07 volt increase in the bias voltage to $V_{B}=0.2792$ volts.

The responsivity and noise properties of the detector are determined from equation (3.1). Rearrangement of equation (3.1) results in a detector resistance of 


$$
R_{D}=\frac{R_{f}}{v_{O U T} / V_{B}-1}
$$

The current $I_{D}$ passing through the detector is given by Ohm's Law to be

$$
I_{D}=\frac{V_{B}}{R_{D}}
$$

The current noise $I_{N}$ from the detector is given by

$$
I_{N}=\frac{V_{N}}{R_{D}}\left(\frac{V_{B}}{V_{\text {OUT }}}\right)
$$

where $V_{N}$ represents the detectors contribution to the total noise voltage from all sources as measured by the noise circuit.

Figure (3.9) is a schematic of the noise circuit that was designed to have an output proportional to the root-mean-square of the voltage fluctuations in the output of the preamplifier. The primary component is an integrated circuit from Analog Devices (type AD536AJD) that acts as a true rms-to-dc voltage converter. To assure that the device was operating in its linear region, an amplifier with a gain of 100 was used on the input stage of the converter. The noise circuit was powered by the same dual 15 volt power supply as the preamplifier.

A germanium resistance thermometer from LakeShore Cryotronics Inc. (type GR-200B-1000) was selected as a temperature measuring device. It was used in a four lead potentiometric configuration 


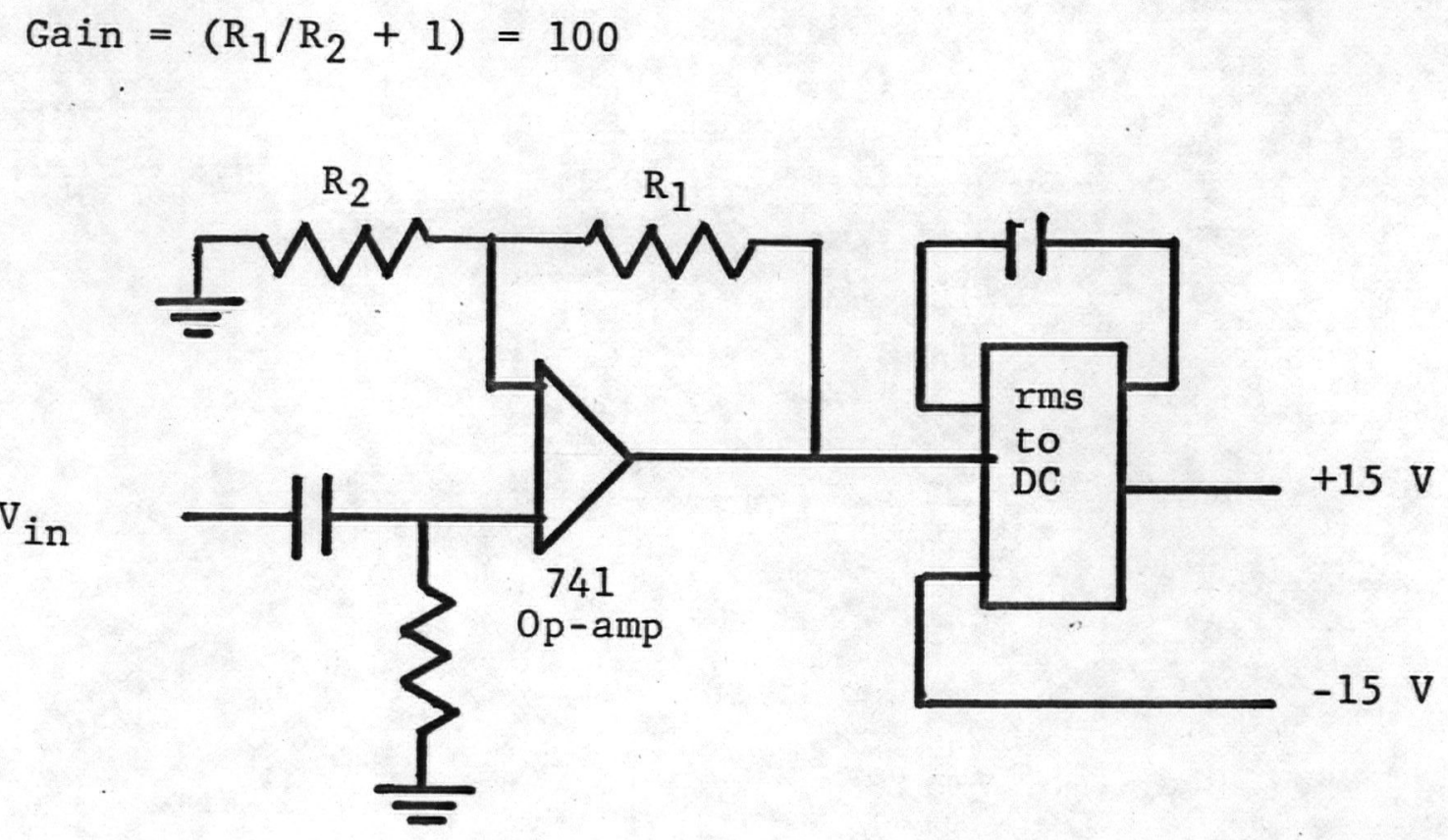

Fig.(3.9) - TRUE RMS TO DC NOISE CIRCUIT WITH A GAIN OF 100 
where two of the leads pass a constant current through the device and the remaining two leads measure the voltage drop across the resistive element. This configuration reduces the resistive heating that would normally be present in a two wire device. The constant currents ranged from $680 \mu \mathrm{A}$ down to $0.76 \mu \mathrm{A}$ to accommodate temperatures from $300^{\circ}$ Kelvin down to $2^{\circ}$ Kelvin.

Two different calibration methods were employed in two different temperature regimes. For temperatures ranging from $4.2^{\circ}$ Kelvin to $40^{\circ}$ Kelvin our thermometer was calibrated against a similar device borrowed from Professors W. Johnson and D. Douglas of the Department of Physics and Astronomy at the University of Rochester. For temperatures below $4.2^{\circ}$ Kelvin, the resistance $R$, of germanium is assumed to increase as

$$
R=R_{0} \exp (A / T),
$$

where $R_{0}$ and $A$ are constants and $T$ is the temperature in degrees Kelvin. This equation is represented by a straight line when plotted on semi-log paper. The two unknown parameters $R_{0}$ and $A$ can be determined by measuring the resistance at two different temperatures. One temperature was obviously $4.2^{\circ}$ Kelvin for which we accurately knew the resistance. The second temperature chosen was the $\lambda$-point of liquid helium which occurs at $2.17^{\circ}$ Kelvin. At its $\lambda$-point, liquid helium ceases to boil and becomes a super fluid. To determine at what point this occurred, a glass plate was placed at the top of the helium reservoir which was being pumped on. The reduced pressure caused the boiling temperature of the helium to drop and the point at 
which it stopped boiling was clearly visible through the plate glass. The values $R_{0}=177$ and $A=6.3$ were determined to correspond to the LakeShore device.

\subsection{Filter and Window Materials}

Polyethylene was selected as the window material for the dewar. It is characterized as having no appreciable absorption bands greater than 14 microns and has a transmission of approximately $80 \%$ between 60 and 100 microns for a thickness of $0.3 \mathrm{~mm} .{ }^{4}$ The polyethylene window used was approximately $1.6 \mathrm{~mm}$ in thickness which resulted in a transmission of about $30 \%$. The window had to be thick in order to resist the force exerted on it by atmospheric pressure when the dewar was under vacuum.

A BPF-2 far infrared band-pass filter made by Descriptive Design and Development Corporation was selected for use at 100 microns. The filter and the polyethylene window material were borrowed from Professor Judith Pipher at the department of Physics and Astronomy at the University of Rochester. Figure (3.10) shows the transmission profile of the filter. Its band-pass is between 44 and 100 microns with a peak transmittance of $42 \%$ at 62 microns. 5 The transmittance of this filter is good between 60 and 90 microns, ranging from $42 \%$ to $25 \%$ respectively. The resulting transmission of the window and filter ranges from a maximum of $12.6 \%$ at 60 microns, $7.5 \%$ at 90 microns, down to zero at 100 microns. 


$$
\text { Un }
$$


It is obvious that this is not the ideal filter for 100 micron detection, but it is adequate in the range from 60 to 90 microns. Unfortunately, filters with a peak transmittance at 100 microns were not available to the author at the time of this research. 


\section{REFERENCES}

1. P.R. Bratt, Ge:Ga and Ge:Be Photoconductive Detectors for Far Infrared Astronomy from a Space Platform, (SPIE Vo1. 132, 1978).

2. L.S. Varnell and D.E. Langford, Radiation Effects in IRAS Extrinsic Infrared Detectors, (Jet Propulsion Laboratory, Pasadena, Ca., June 1982).

3. W.J. Moore, 100 Micron Detector Development Program, (Ames Research Center, NASA, March 1976).

4. Y. Yamamada, Transmission Filters in the $\times$ FarInfrared Region, (Journal of the Optical Society of America, Vol. 52 no.1, January 1962).

5. S.P. Varma, Far Infrared Band-Pass Filters in the 400-16 $\mathrm{cm}^{-1}$ Spectral Region, (Applied Optics, Vol. 8, October 1969) page 2151.

6. P. Horowitz and W. Hill, The Art of Electronics, (Cambridge University Press, 1980). 


\section{CHAPTER 4}

EXPERIMENTAL RESULTS

\subsection{Introduction}

Test procedures which led to system optimization are discussed in the first section of this chapter. The second section presents experimental data for the dark current as a function of the detector temperature. A comparison is made to the theoretical predictions presented in chapter 2. This is followed by a comparison of theory and experiment for the mean-square current noise as a function of temperature. Finally, data is presented for the current responsivity, both as a function of the blackbody temperature and the total power incident on the detector.

\subsection{Initial Testing and System Optimization}

System optimization focused primarily on the preamplifier and sources of undesirable noise. As was discussed in chapter 3 , the 
inefficient vacuum system slowed the optimization process considerably.

During the first run of the experiment, the preamplifier output remained saturated at approximately $14.45 \mathrm{~V}$. Two plausible explanations came to mind; (1) the feedback resistance was too high and (2) the preamplifier would not function properly at $77^{\circ}$ Kelvin.

A Keithley Instruments 601 Electrometer was employed to measure resistances of the order of $10^{8}$ to $10^{10}$ ohms. A resistance of $4.4 \times 10^{8}$ ohms, which was thought to be lower than the desired feedback resistance, was used on the subsequent trial to assure that the preamplifier gain would not be saturated.

Evaluation of the test circuit revealed that the LM108 operational amplifier would not function at $77^{\circ}$ Kelvin. Thus, the operational amplifier was mounted to a room-temperature copper bracket that was soldered to the inside of the helium cryostat.

The next run of the system proved to be successful and data indicated that the detector resistance was on the order of $10^{8}$ ohms at $4.2^{\circ}$ Kelvin. Three of the feedback resistors acquired from Jet Propulsion Laboratory were connected in parallel, resulting in a feedback resistance of $R_{f}=4 \times 10^{9}$ ohms. This resistance resulted in voltage saturation of the amplifier at a temperature of $6^{\circ}$ Kelvin.

Considerable effort was devoted to locating and minimizing sources of undesriable noise. Unwanted noise resulted from mechanical vibrations, electrical pickup, and ground loops. The last two problems were cured by shielding all signal wires and bringing all ground leads to a common point. The reason for the appreciable sen- 
sitivity to mechanical vibrations was never completely understood. It may arise from compression of the crystal lattice resulting in a voltage change associated with the piezo-electric effect. Four inertubes were placed beneath a 250 pound steel plate on which the cryostat was magnetically mounted. This served to isolate the detector from the mechanical vibrations of its environment. The isolation table was effective, but did not entirely eliminate the problem of mechanical pick-up.

\subsection{Experimental Results}

The dark current as a function of the detector temperature was determined from the preamplifier output data by use of equations (3.2) and (3.3). The detector temperature ranged between $2^{\circ}$ and $5^{\circ}$ Kelvin. A high volume vacuum pump was connected to the helium reservoir to permit temperatures below $4.2^{\circ}$ Kelvin to be reached. A $1 \mathrm{k} \Omega$ power resistor attached to the detector holder and connected to

a $30 \mathrm{~V}$ power supply was employed to reach temperatures in excess of $4.2^{\circ}$ Kelvin. Heating due to the power resistor resulted in a temperature gradient between the LakeShore thermometer and the detector element. The experimental data above $4.2^{\circ}$ Kelvin was thus shifted up in temperature resulting in a discontinuous curve when plotted. Decreasing the experimental values of the temperature by $1 / 3^{\circ}$ Kelvin for data taken with the power resistor on resulted in a continuous function. These corrected data are assumed to be the true experimental values and are used in the following analysis. 
The theoretical value of the mean current passing through the detector is given by equation (2.21) to be

$$
\bar{i}=\frac{e V}{L^{2}}\left[\mu_{n} \bar{N}+\mu_{p} \bar{P}\right] .
$$

Since gallium is an acceptor impurity in Ge:Ga, $\mu_{n} \bar{N}$ will be much less than $\mu_{p} \bar{p}$. The resulting theoretical detector current is given by

$$
\bar{i}=\frac{e V}{L^{2}} \mu_{p} \bar{P},
$$

which is expressed in terms of the fractional ionization as

$$
\bar{i}=\frac{e V}{L^{2}} \mu_{p}\left(N_{a}-N_{d}\right) f .
$$

The following material parameters for the Ge:Ga detectors used in this experiment are quoted from a report by Jet Propulsion Laboratory: ${ }^{1}$

$$
\begin{aligned}
& \mu_{p}=50 \mathrm{~m}^{2} \mathrm{v}^{-1} \mathrm{sec}^{-1} \\
& L=1.4 \times 10^{-3} \mathrm{~m} \\
& \mathrm{~N}_{\mathrm{d}}=1.58 \times 10^{9} \text { donors } \\
& \mathrm{N}_{\mathrm{a}}=7.88 \times 10^{11} \text { acceptors. }
\end{aligned}
$$

The detector bias as stated in chapter 3 was $V_{B}=0.2792 \mathrm{~V}$. These parameters result in a theoretical current given by

$$
\bar{i}=(0.9) f[A] \text {, }
$$


where again $f$ is the fractional ionization of available acceptor impurities. Figure (4.1) illustrates the fractional ionization versus temperature in Ge:Ga between the temperatures of $0^{\circ}$ and $50^{\circ}$ Kelvin. Note that $f$ begins to saturate at about $10^{\circ}$ Kelvin.

Experimental data for the detector dark current in nanoamps versus the detector temperature in degrees Kelvin is indicated by the octogonal marks in figure (4.2). The solid lines represent the best theoretical predictions of the dark current as explained below. Equation (2.34) gives the functional dependence of the fractional ionization on the thermal generation rate as

$$
f=\left(\frac{1}{1-\lambda}\right)\left\{\frac{-1}{2}\left(\Gamma_{\text {th }}+\lambda\right)+\left[\frac{1}{4}\left(\Gamma_{\text {th }}-\lambda\right)^{2}+\Gamma_{\text {th }}(1-\lambda)\right]^{\frac{1}{2}}\right\}
$$

where the thermal generation rate is given by equation ( 2.41 ) to be

$$
\Gamma_{\text {th }}=\Gamma_{0}\left(k T / \varepsilon_{a}\right)^{3 / 2} \exp \left(-\varepsilon_{a} / k T\right) \text {. }
$$

The material dependent parameter $\Gamma_{0}$ is given by equation $(2.40)$ to be

$$
\Gamma_{0}=\frac{v}{N_{a}}\left(\frac{2 \pi m \varepsilon}{h^{2}}\right)^{3 / 2}
$$

It should be noted that our experimental data for the dark current as a function of temperature does not match perfectly with the theoretical predictions. There are, however, distinct correlations between experiment and theory as is evidenced by figure (4.2). Careful consideration must be given as to how these differences may have come about. Note that equation (4.7) contains two free para- 


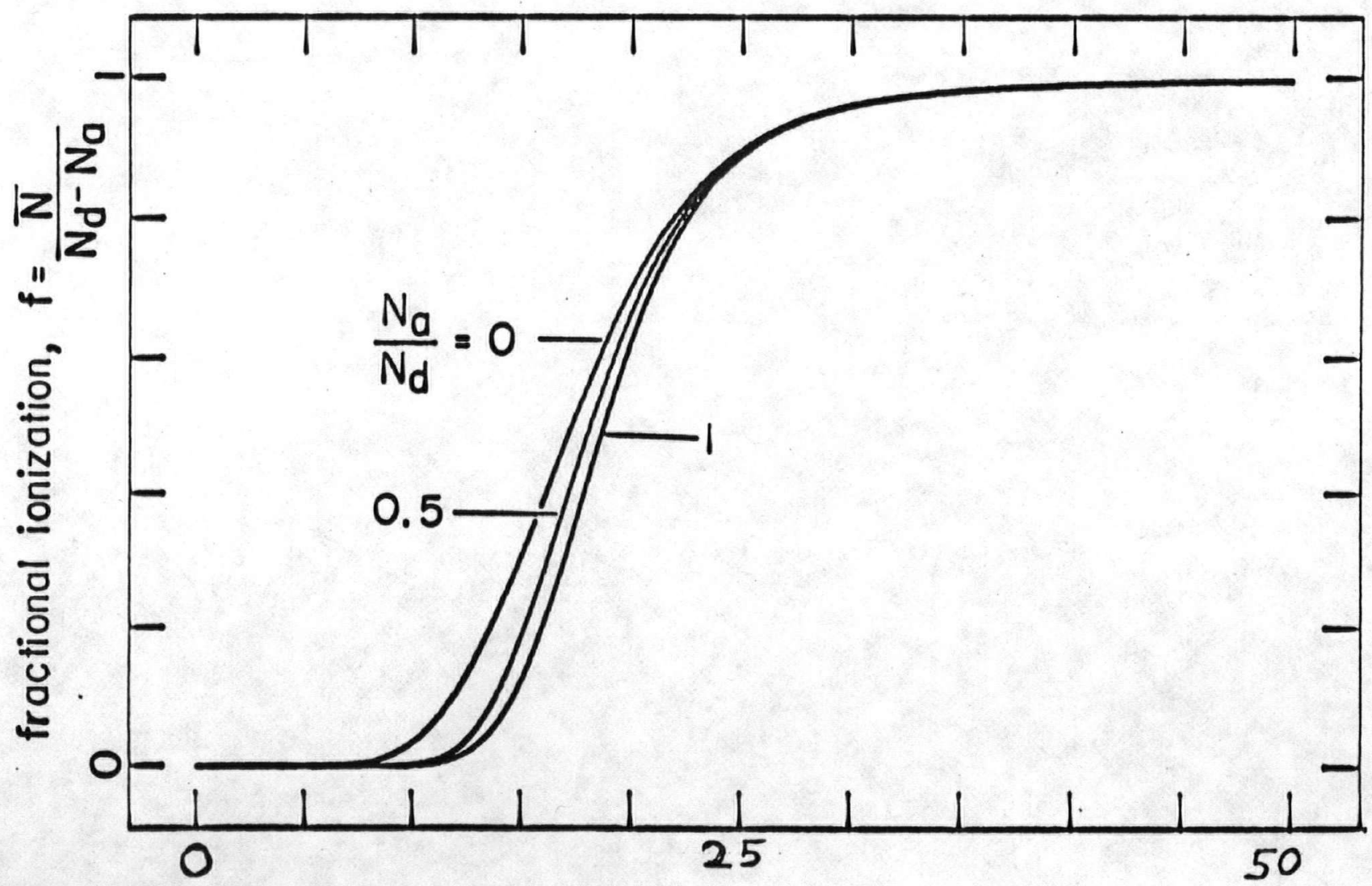

TEMPERATURE (DEGREES KELVIN)

Fig.(4.1) - Fractional ionization versus temperature. 


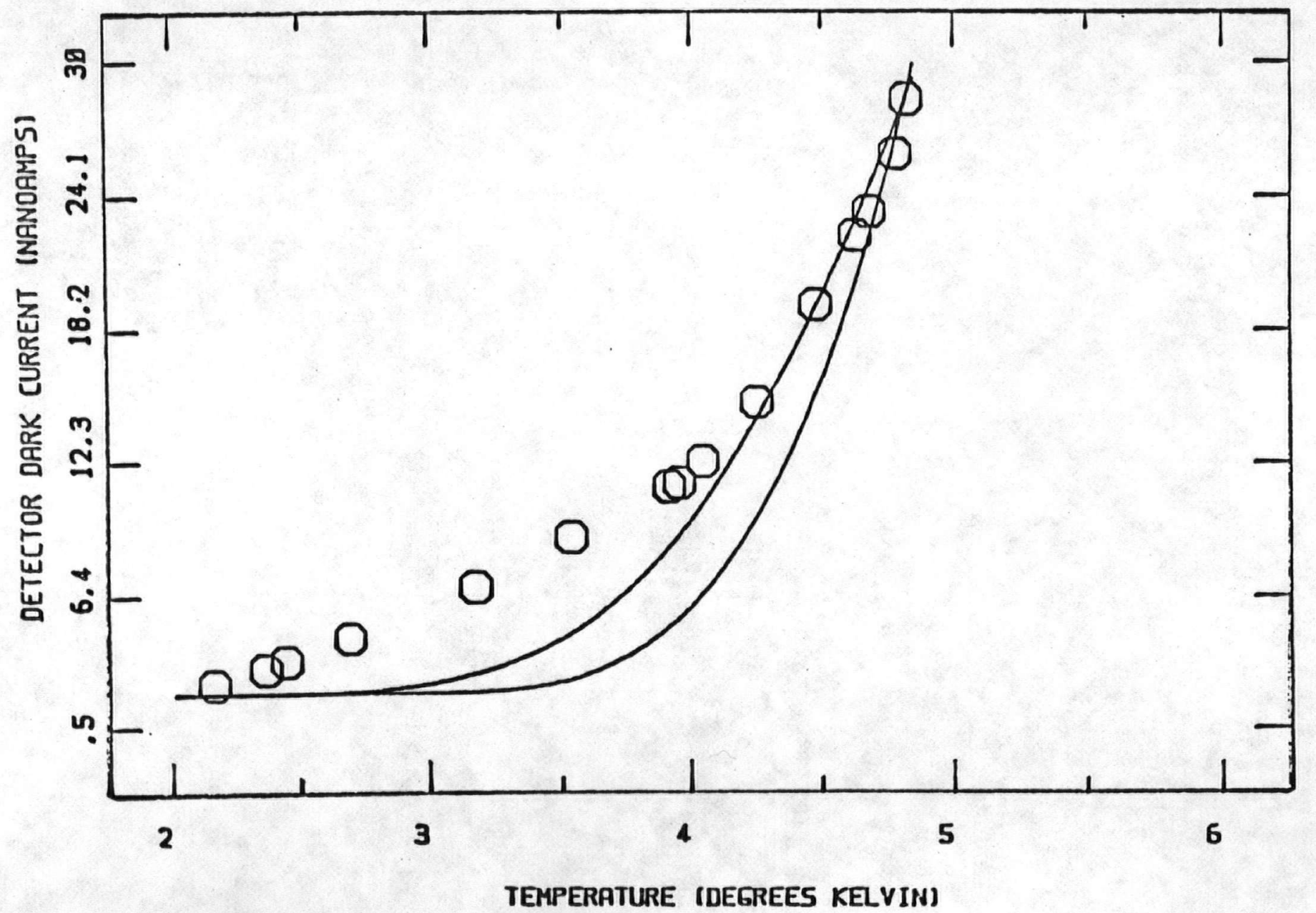

Fig.(4.2) - Dark current versus temperature. 
meters; the acceptor density $\mathrm{N}_{\mathrm{a}} / \mathrm{V}$ and the ionization energy $\varepsilon_{\mathrm{a}}$. Equation (4.7) is exponentially dependent on the ionization energy $\varepsilon_{a}$. Thus, even a small change in this value will produce a noted effect on the fractional ionization. Perhaps it is more accurate to consider the acceptor level to span over a finite energy band rather than a discrete energy level. With this in mind, the lower curve in figure (4.2) represents the ionization energy value of $\varepsilon_{a}=0.0108 \mathrm{eV}$ and the upper solid line represents a $35 \%$ decrease in this value. The resulting curve is seen to conform more closely to the experimental data. Changing the number of acceptors had a much smaller effect and is not shown.

A constant current was also added to the theoretical curve which could be manifested by phenomenon such as leakage current, background photon flux, impact ionization, or thermal conduction through the detector leads. Due to the high resistance of the detector, even a fingerprint could result in a considerable leakage current. The detector leads could also be responsible for a leakage current. A contribution to the current could also result from the background photon flux from the cold shield which is at $77^{\circ}$ Kelvin, a temperature considerably higher than that of the detector. The bias voltage and the inter-electrode distance lead to an electric field strength of approximately $2 \mathrm{~V} / \mathrm{cm}$. According to a report from Santa Barbara Research Center, ${ }^{2}$ impact ionization occurs at an electric field strength on the order of $3 \mathrm{~V} / \mathrm{cm}$ for these detectors. This phenomenon may have resulted in the ionization of an appreciable number 
of acceptors, which would lead to a decrease in the resistance of the detector and thus an increase in the current.

It is important to point out that temperature readings between $4.2^{\circ}$ and $2.17^{\circ}$ Kelvin may be slightly in error, as our assumption of the functional form of the resistance in this domain may not be entirely correct.

We have introduced a number of probable causes for the discrepancies between our experiment and theory, but it is difficult to tell which of these factors plays a dominant role, as their effects are often indistinguishable from each other.

As data was available for similar Ge:Ga detectors from Santa Barbara Research Center, ${ }^{3}$ it seemed reasonable to compare the data to our theory. This comparison is illustrated in figure (4.3). Their detectors were under a background flux of $1.2 \times 10^{9}$ photons $\mathrm{sec}^{-1} \mathrm{~cm}^{-2}$ which is manifest by a constant current contribution. The lower solid curve results from a best estimation of the material parameters and the upper solid curve results from a $50 \%$ decrease in the value of the ionization energy. These curves appear to be in good agreement with our theory.

Figure (4.4) illustrates a comparison between experimental data and theory for the mean-square dark current noise as a function of detector temperature. It should be pointed out that this data did not come from the same detector as the one from which the responsivity data came. Again, the lower curve represents the theory based on the best estimation of material parameters. The upper solid curve 


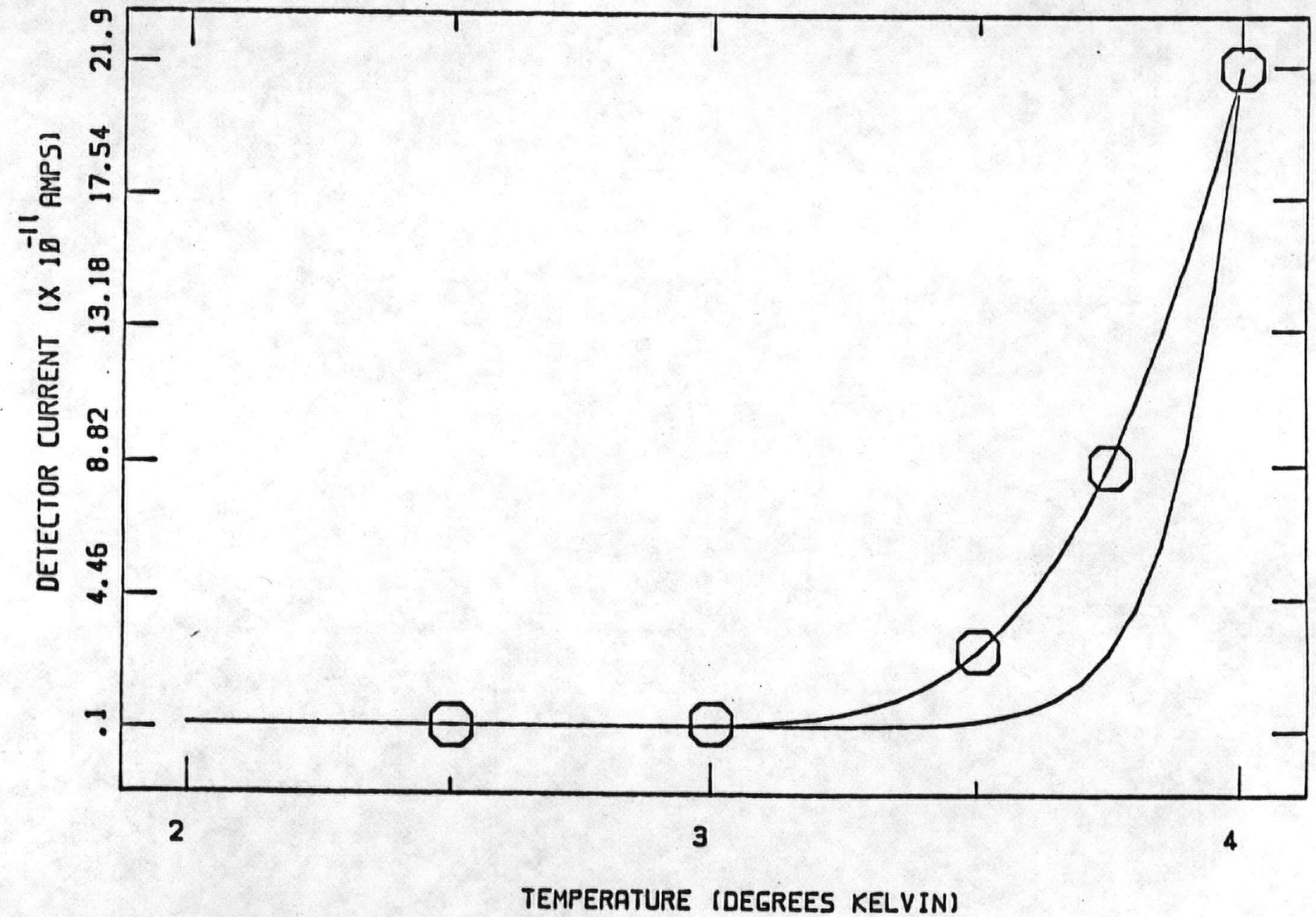

Fig. (4.3) - SBRC data for dark current versus temperature. 


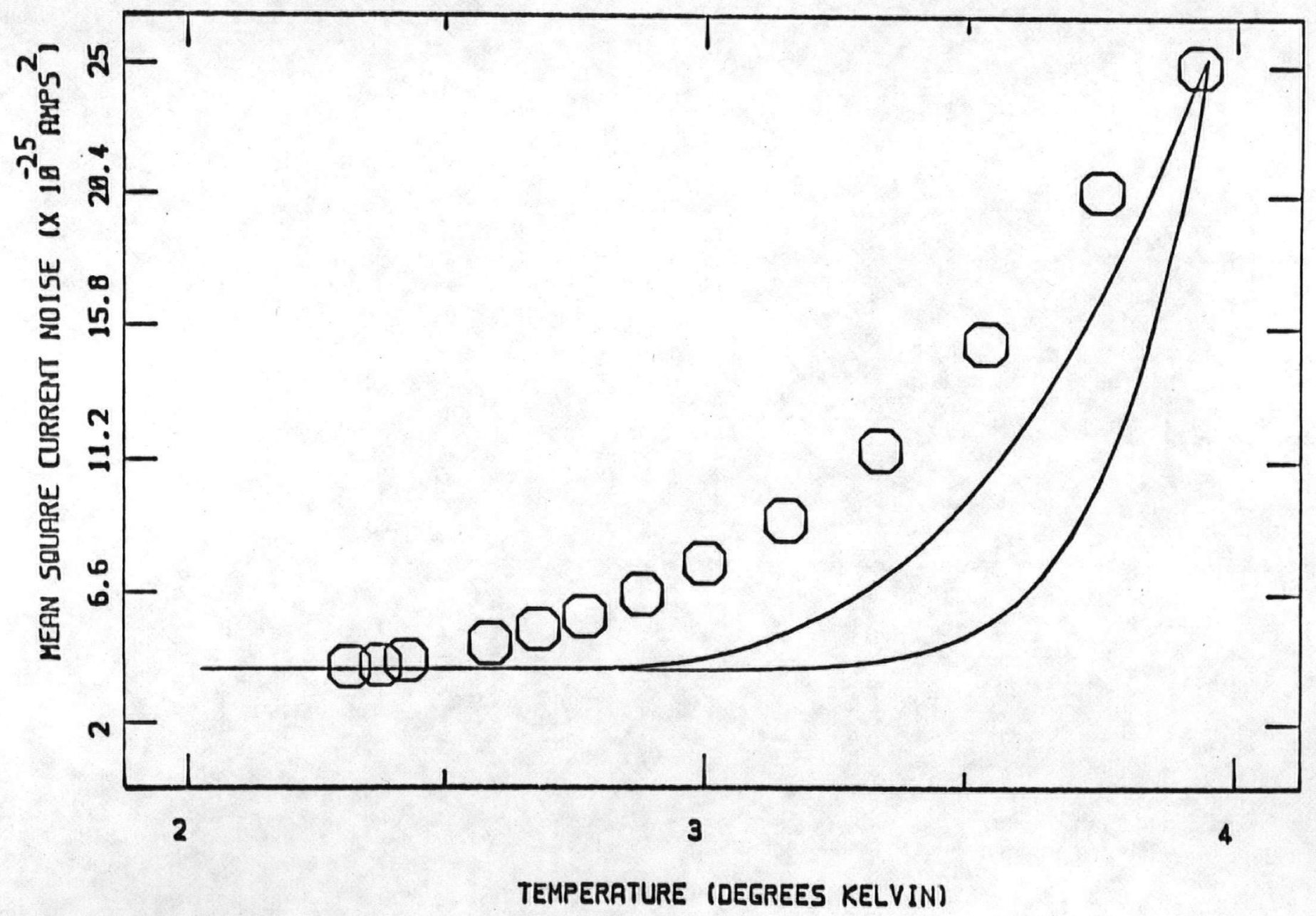

Fig.(4.4) - Mean-square dark current noise versus temperature. 
results from decreasing the value of the ionization energy by $35 \%$ from that of the lower curve.

A constant term is added to the theoretical curves in figure (4.4) to account for such noise sources as amplifier noise, electrical pick-up, and mechanical vibrations. The only appreciable noise from the amplifier was due to the Johnson noise of the bias resistor which amounted to $1.4 \times 10^{-25} \mathrm{~A}^{2}$. This would not effect the shape of the curve, but would increase the current by a constant additive factor. Electrical pick-up was minimized by proper shielding of the electronics and the electrical cables. As mentioned above, noise due to mechanical vibrations was reduced by the isolation table, but was still evident. It is assumed that these fluctuations average over time to result in a constant current noise contribution.

The Johnson noise due to the detector and load resistances does lead to a non-constant contribution to the noise current. This could play an important role in the differences between theory and experiment. This noise current is given by ${ }^{4}$

$$
\bar{i}_{N_{\text {Johnson }}^{2}}=\frac{4 k T}{R_{d}}+\frac{4 k T}{R_{f}} .
$$

The Johnson noise due to the feedback resistor increases only with an increase in detector temperature. The Johnson noise associated with the detector resistance, however, increases with an increase in detector temperature and with the associated decrease in detector resistance. This noise contribution reaches a maximum of only 
$3 \times 10^{-29} A^{2}$ due to the low detector temperature and to the high detector and feedback resistances. Thus it does not constitute a large contribution to the total noise.

The final stage of experimentation involved photon flux responsivity measurement as a function of blackbody temperature and the total power incident on the detector. A blackbody source at a distance of 7 inches and having an aperature of 1 inch in diameter was varied between the temperatures of $473^{\circ}$ and $1073^{\circ}$ Kelvin. The blackbody had to be positioned as close to the dewar window as possible because of the high absorption of 100 micron radiation by water molecules. This requirement presented difficulties in collecting good data due to the inevitable heating of the cryostat and detector. Due to the large photon flux on the detector, detector holder, and work surface, temperatures below $3.3^{\circ}$ Kelvin could not be reached. Two data points were taken at each blackbody temperature. One was taken with a mirror in front of the window to monitor the dark current and one measurement was taken without the mirror to determine the flux responsivity. Note that the dark current increased with increasing blackbody temperature due to heating of the detector.

To determine the responsivity it is first necessary to calculate the power $P$ incident on the detector. The power is given by

$$
P=L A F \Omega,
$$

where $L$ is the radiance in a wavelength interval between 60 and 90 microns, $A$ is the effective area of the detector, $F$ is the average 
transmittance of the air, window, and filter $(F=0.05)$, and $\Omega$ is the limiting solid angle. The integrating cavity defines a solid angle of approximately $2 \times 10^{-2} \mathrm{sr}$. The blackbody aperature subtends a solid angle of only $1.5 \times 10^{-2} \mathrm{sr}$. The power falling on the detector must include both a contribution due to the blackbody and a contribution due to the $300^{\circ}$ Kelvin background.

The total radiance is given by $L=M / \pi$ where $M$ is the radiant exitance given by

$$
M=\sigma T^{4},
$$

where $\sigma$ is known as the Stephan-Boltzman constant and is equal to $5.67 \times 10^{-8} \mathrm{~W} / \mathrm{m}^{2} \mathrm{~K}^{4}$, and $T$ is the temperature in degrees Kelvin. The fraction of the total radiance contained between the wavelengths of 60 and 90 microns was calculated from a table of the Planck function ${ }^{5}$ for a given blackbody temperature. The power due to the $300^{\circ} \mathrm{Kelvin}$ background was found to be approximately $4.5 \times 10^{-8} \mathrm{~W}$.

We assume that the current responsivity $R$ is of the form

$$
R=\frac{I_{p h}-I_{t h}}{P_{T}},
$$

where $I_{p h}$ is the current in the presence of the photon field, $I_{t h}$ is the dark current, and $P_{T}$ is the total power incident on the detector. Figures (4.5) and (4.6) represent the increase in the current through the detector due to photon flux as functions of the blackbody temperature and the total incident power, respectively. Notice that the total incident power and the blackbody temperature scale by approxi- 


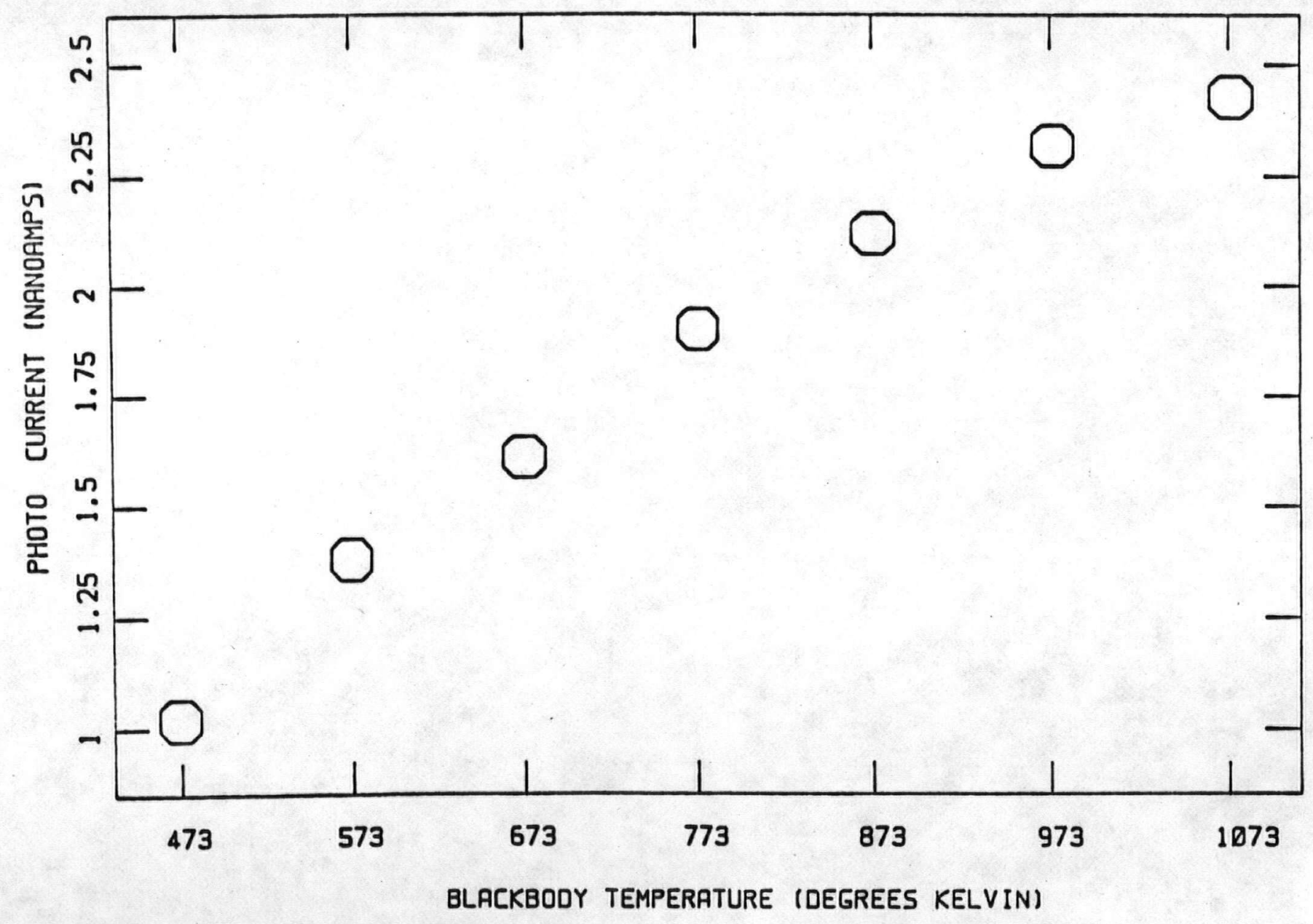

Fig.(4.5) - Photocurrent versus blackbody temperature. 


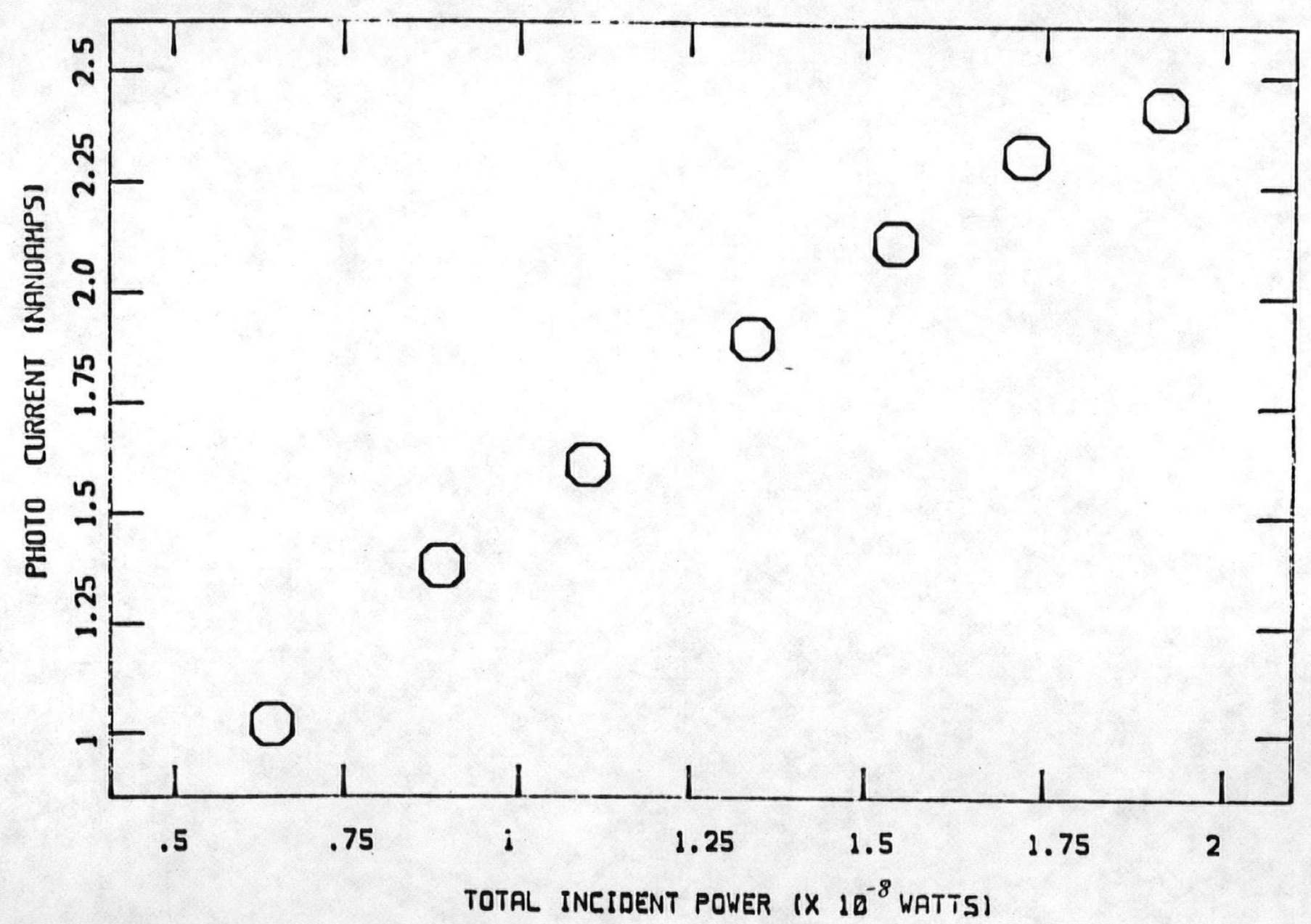

Fig.(4.6) - Photocurrent versus total incident power. 
mately equal amounts with respect to the photo-induced current. This is a consequence of the Rayleigh-Jeans radiation law in the limit hv $\ll k T$. Current responsivity is plotted as functions of the blackbody temperature and the total incident power in figures (4.7) and $(4.8)$, respectively.

These four plots appear to be in agreement with theory. If we look at figure (2.4) of the fractional ionization as a function of the dimensionless generation rate, we see a functional dependence similar to that of figures (4.5) and (4.6). Again, the fractional ionization is proportional to the current. The dependence of the dimensionless generation rate on the photon flux is not known, as we do not know the functional form of the recombination coefficient $p$. The responsivity in figures (4.7) and (4.8) is seen to decrease with increasing flux, a dependence which is supported by figure (2.21) of the responsivity as a function of the dimensionless generation rate $\Gamma$.

The noise equivalent power, or NEP, was measured under conditions in which the detector was viewing a $473^{\circ}$ Kelvin blackbody source. The NEP is given by

$$
N E P=\frac{I_{N}}{R}
$$

According to the theory presented in chapter 2, the current noise does not distinguish between photon and thermal generation rates of equal magnitude. It only depends on the magnitude of the dimensionless generation rate $\Gamma$. Thus, the noise current from previous dark 


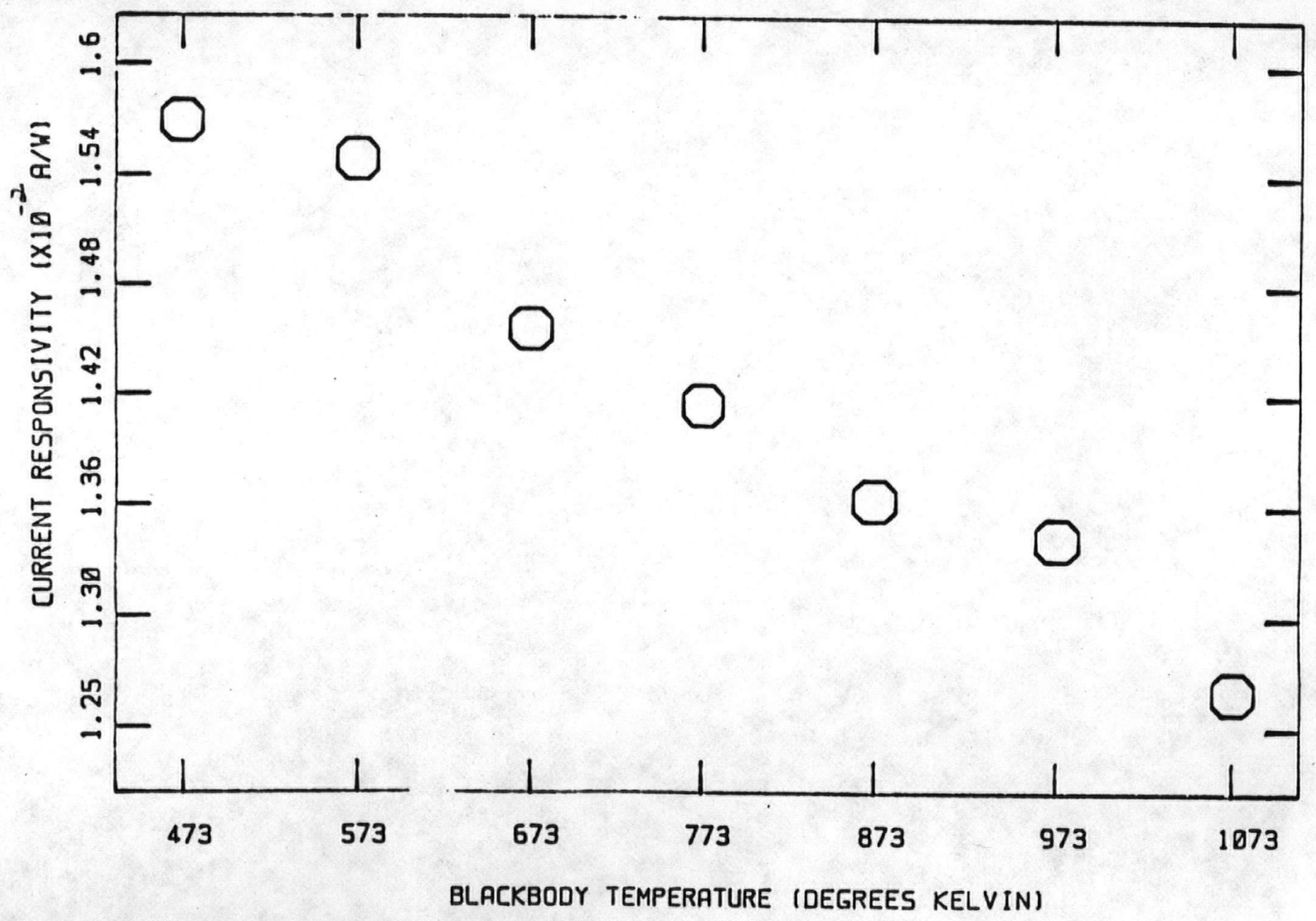

Fig.(4.7) - Current responsivity versus blackbody temperature. 


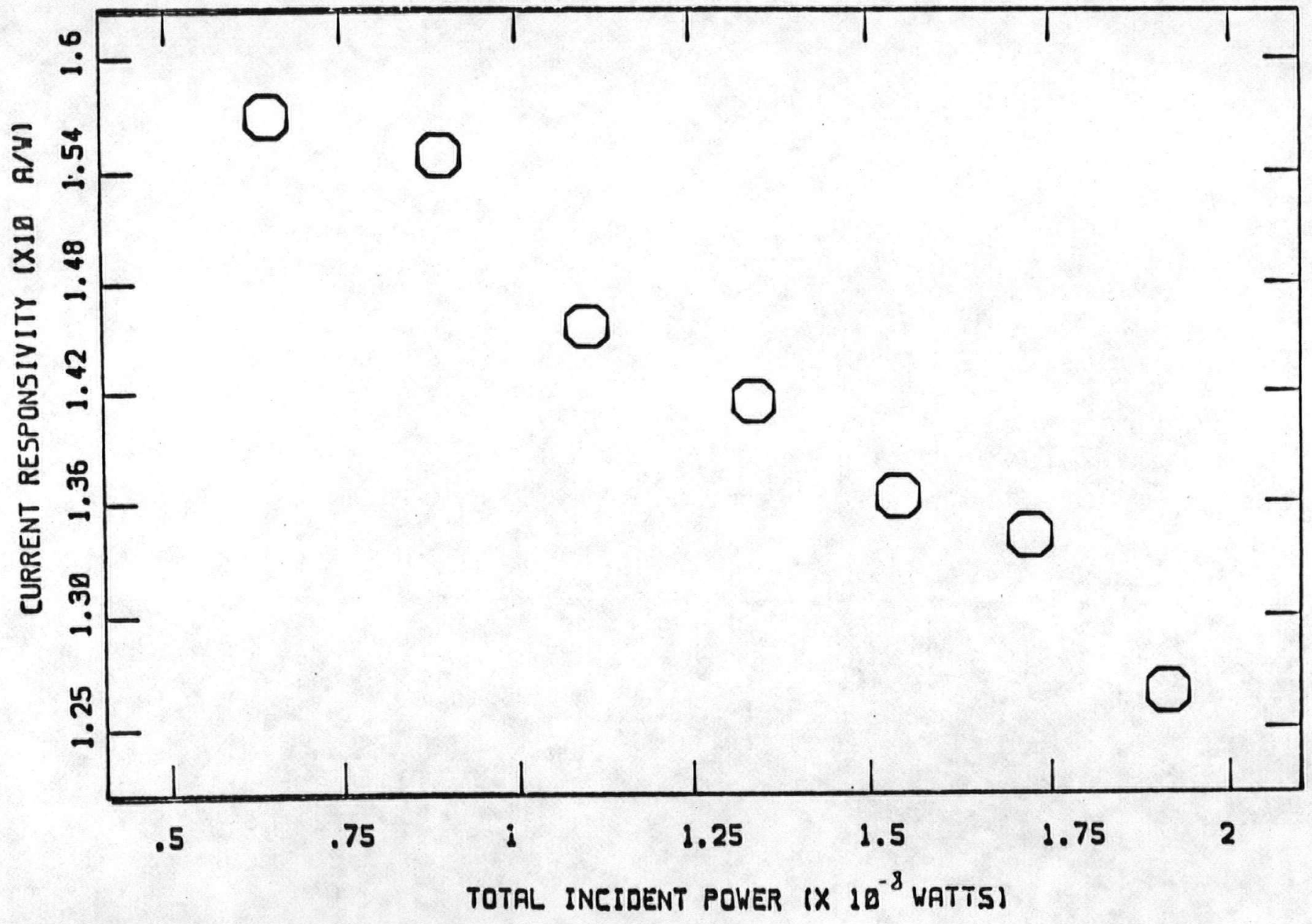

Fig.(4.8) - Current responsivity versus total incident power. 
current measurements could be used to calculate the NEP from the responsivity measurements described above. The value of the resistance of the detector used with a $473^{\circ}$ blackbody was used to determine the proper current noise. The effect due to photon bunching was not considered in this calculation. The current noise was determined to be $I_{N}=1.8 \times 10^{-12} \mathrm{~A}$. This results in an $\mathrm{NEP}=1.15 \times 10^{-10} \mathrm{~W} / \sqrt{\mathrm{Hz}}$. It is informative to compare this NEP to the background limited NEP given by

$$
{ }_{N E P}{ }_{B L}=\left[\frac{{ }^{4 P_{B} h \nu \Delta f}}{\eta}\right]^{\frac{1}{2}} \text {, }
$$

where $P_{B}$ is the background power, $h$ is Planck's constant, $v$ the mean optical frequency, $\Delta f$ is the electrical bandwidth, and $\eta$ is the quantum efficiency. The background power of $4.5 \times 10^{-8} \mathrm{~W}$ results in a background limited NEP of $\mathrm{NEP}_{\mathrm{BL}}=1.1 \times 10^{-12} \mathrm{~W} / \sqrt{\mathrm{Hz}}$. As was expected from the theory presented in chapter 2 , the measured value of the NEP is above that calculated for the theoretical limit. Part of the discrepancy may be due to the extremely high flux level which would lead to a lower responsivity and higher current. There is also some uncertainty associated with the photon flux level. Note that the above analysis is in general qualitative agreement with the theory as presented in chapter 2. 
90

REFERENCES

1. Reference 2, Chapter 3, page 6.

2. Reference 1, Chapter 3, page 99.

3. Reference 1, Chapter 3, page 103, Figure 8.

4. Reference 6, Chapter 2, pp. 136-141.

5. R.D. Hudson, Infrared System Engineering, (Wiley, New York, 1969). 
CHAPTER 5

SUMMARY AND CONCLUSIONS

This thesis has presented a complete theory of the generation- recombination noise associated with extrinsic photoconductive detectors. The statistical approach of past treatments has been employed in greater generality and scope. Our treatment has allowed for both the thermal and photo-excitiation of charge carriers. The analysis also accounted for the impurity compensation effect on detector response characteristics. The method of detailed balancing, and Master equation techniques were used to derive closed form equations of several detector response characteristics. Chapters 3 and 4 described a Ge:Ga detection system. The experimental setup and system optimization were discussed in detail. Experimental measurements of the detector's response characteristics were found to be in good qualitative agreement with the theory developed in chapter 2 . 